\title{
Experimental, Computational and Analytical Studies towards a Predictive Scenario for a Burning Accident
}

\author{
Furkan Kodakoglu \\ fk0011@mix.wvu.edu
}

Follow this and additional works at: https://researchrepository.wvu.edu/etd

Part of the Heat Transfer, Combustion Commons

\section{Recommended Citation \\ Kodakoglu, Furkan, "Experimental, Computational and Analytical Studies towards a Predictive Scenario for a Burning Accident" (2020). Graduate Theses, Dissertations, and Problem Reports. 7669. \\ https://researchrepository.wvu.edu/etd/7669 \\ This Dissertation is protected by copyright and/or related rights. It has been brought to you by the The Research Repository @ WVU with permission from the rights-holder(s). You are free to use this Dissertation in any way that is permitted by the copyright and related rights legislation that applies to your use. For other uses you must obtain permission from the rights-holder(s) directly, unless additional rights are indicated by a Creative Commons license in the record and/ or on the work itself. This Dissertation has been accepted for inclusion in WVU Graduate Theses, Dissertations, and Problem Reports collection by an authorized administrator of The Research Repository @ WVU. \\ For more information, please contact researchrepository@mail.wvu.edu.}


Experimental, Computational and Analytical Studies towards a Predictive Scenario for a Burning Accident

\author{
Furkan Kodakoglu
}

\author{
Dissertation submitted to the \\ Statler College of Engineering and Mineral Resources \\ at West Virginia University \\ in partial fulfillment of the requirements \\ for the degree of \\ Doctor of Philosophy \\ in \\ Mechanical Engineering
}

V'yacheslav Akkerman, Ph.D., Chair

Hailin Li, Ph.D.

Cosmin Dumitrescu, Ph.D.

Derek R. Johnson, Ph.D.

Ali S. Rangwala, Ph.D.

Department of Mechanical and Aerospace Engineering

West Virginia University

Morgantown, West Virginia

August, 2020

Keywords: vented gas explosion, explosion safety, flame acceleration

Copyright 2020 Furkan Kodakoglu 


\begin{abstract}
Experimental, Computational and Analytical Studies towards a Predictive Scenario for a Burning Accident

Furkan Kodakoglu
\end{abstract}

Historically, accidental gas and dust explosions constitute one of the major hazards to both personnel and equipment in the process industries. The current knowledgebase on such explosions does not provide an acceptable level of risk. Therefore, novel preventive mining/fire safety strategies, based on a rigorous predictive scenario for burning accidents, are critically needed. The present dissertation is devoted to such a predictive scenario, with a particular focus on the flame and pressure evolutions in explosions encountered in an enclosure with or without obstructions.

The experimental component of this dissertation comprises a series of experiments on explosion venting. Specifically, the influence of the vent area on the overpressure and dynamics of the fuellean, stoichiometric, and fuel-rich methane-air flames was studied. First, the experiments were conducted in a transparent polycarbonate cylindrical chamber to allow for real-time visualization of the flame front. Experimental parameters included ignition location, central or rear, and three various vent areas (with negligible vent relief pressure). As expected, the highest maximum pressure was associated with the stoichiometric conditions and the smallest vent area. For a fuelrich mixture with central ignition, a flashback phenomenon was observed after an external explosion. The experimental study was subsequently extended to a twice longer cylinder (with only rear ignition). It showed that an increase in the length of the cylinder promotes the overpressure and the acceleration rate. An engineering model to predict the pressure-time histories of stoichiometric methane-air vented deflagrations was updated and compared to the experiments. Good agreement between the experiments and the simulations was obtained in terms of pressure rise and peak pressure predictions. The future work was recommended on further development of the model in larger scales, congested volumes, and multi-compartment enclosures.

For future development of the model, the mechanisms of flame propagation in the passages with or without obstructions were studied. First, the assumptions used on finger flame acceleration were reviewed. The mechanistic and thermal impacts of the passage walls on finger flame acceleration were studied by means of the fully-compressible computational simulations of the reacting flow equations. It was shown that the difference between the effects of slip and nonslip walls was generally minor during the acceleration stages of burning. After a flame skirt contacted a sidewall, wall friction played a role and promoted the flame further. As for the thermal boundaries, cold isothermal walls cool down the flame skirt.

Within the theoretical component of this dissertation, the theory for a globally-spherical, selfaccelerating expanding premixed flame front was combined with that of extremely fast flame acceleration in obstructed conduits to form a new analytical formulation. The coalmining geometry is imitated by two-dimensional and cylindrical passages of high aspect ratio, with a comb-shaped array of tightly-placed obstacles attached to the walls. Specifically, the key stages of premixed flame front evolution were identified and scrutinized, by quantifying their major characteristics such as the flame tip position and its velocity. Starting with an incompressible assumption, the analysis was then extended to account for gas compressibility, because the latter cannot be ignored as soon as the burning velocity starts approaching the speed of sound. It was shown that the effect 
of gas compressibility moderates flame acceleration, and such an impact depends strongly on various thermal-chemical properties of the combustible mixture. The theoretical investigation of the problem revealed that the influence of both the obstacles and the combustion instability on the fire scenario was substantial, and this effect grew stronger with the blockage ratio. Starting with gaseous methane-air combustion, the formulation was subsequently extended to gaseous-dusty environments. Specifically, the coal (combustible, i.e. facilitating the fire) and inert (such as sand, moderating the process) dust and their combinations were considered. The impact of the size and concentration of the dust particles on flame acceleration was quantified. Eventually, the analytical predictions were compared with the experiments and the numerical simulations from the literature, with good agreement obtained. Finally, the comparison of the theory, simulations and experiments of this dissertation was conducted in terms of the exponential acceleration rates, with qualitatively good agreement demonstrated. 


\section{Dedication}

To The Kodakoglus; Bulent Kodakoglu, Adalet Kodakoglu and Alper Kodakoglu. 


\section{Acknowledgments}

Before anything, I express my gratitude to Allah who made everything possible.

I would like to thank my advisor, Dr. Akkerman, who accepted me as his graduate student and gave me the opportunity to work on this special project. Throughout my time at West Virginia University (WVU), your support was always encouraging to complete this work. I would like to express my thankfulness to my committee members; Dr. Li, Dr. Dumitrescu, Dr. Johnson and Dr. Rangwala, for their constant desires and efforts to improve my dissertation. A special thanks to Dr. Rangwala, for giving me the opportunity to work in his team and allowing me to use the resources of his Combustion Lab at the Fire Protection Engineering (FPE) Department of the Worcester Polytechnic Institute (WPI) to complete my work and advising me in my research.

I would like to also thank Mohammed AlKhabbaz, Dr. Demir, Dr. Farahani, Dr. Sezer and Dr. Valiev for their contribution to this work. My thanks also go to all the past and current members of Dr. Akkerman's research group at WVU as well as the researchers in the combustion lab at WPI. Special thanks go to Samuel Ogunfuye for his help in the final stages of my work. Deceased Dr. Bychkov, whose research was very helpful, should be also recognized and taken great advantage of throughout my work.

Being a part of WVU was and will always be my privilege. I greatly appreciate all the support provided to me by the Department of Mechanical and Aerospace Engineering. I would like to thank my friends: Baris Sevi, Filiz Kazan, Mustafa Can Suner and Denizes, for their support when I fell.

Last, but not least, I am grateful to be a part of my family and relatives.

This work has been funded by the Alpha Foundation for the Improvement of Mine Safety and Health, Inc., through Award \#ASTI14FO-82, prior to which it was funded by the U.S. National Science Foundation (NSF) through CAREER Award No. 1554254 as well as by the West Virginia Higher Education Policy Commission through Grant \#HEPC.dsr.18.7. 


\section{Table of Contents}

Abstract

Dedication iv

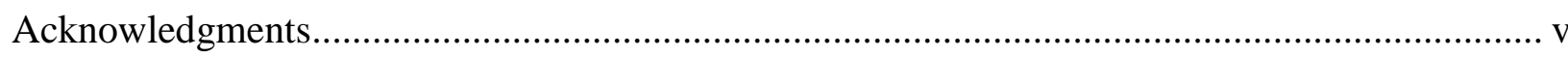

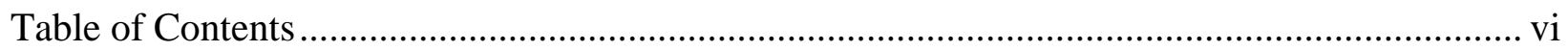

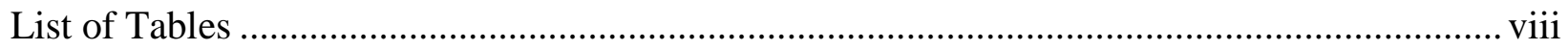

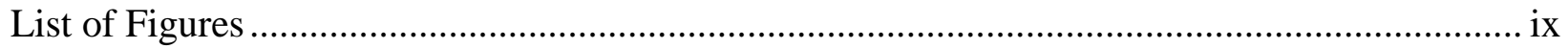

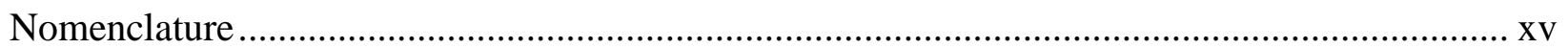

1. Introduction: Combustion Science - the State of the Art ..................................................... 1

1.1. Laminar Burning Velocity of Premixed Gas/Vapor and Air ...................................... 2

1.2. Maximum Pressure Generated from Combustion of Premixed Gas/Vapor and Air at

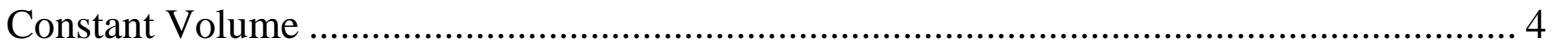

1.3. Thermal Expansion Ratio of Premixed Gas/Vapor and Air ....................................... 5

1.4. The Darrieus-Landau Instability of a Thin Flame Front ........................................... 7

1.5. Safety in Industry - a Persistent Challenge ….................................................... 8

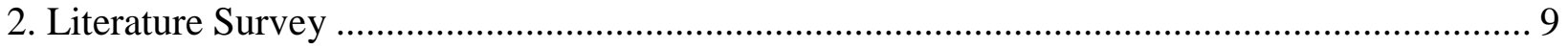

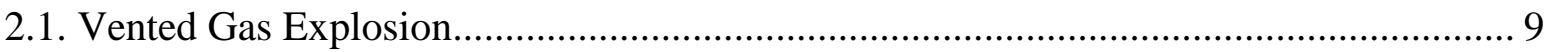

2.2. Flame Acceleration - the Effect of Heat Loss ....................................................... 22

2.3. Fire Scenario in an Obstructed Mining Passage ................................................. 25

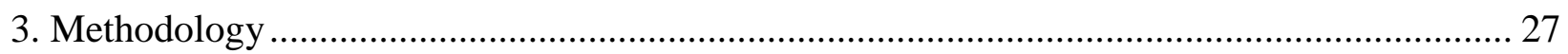

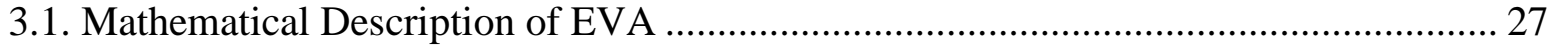

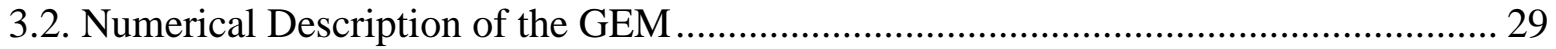

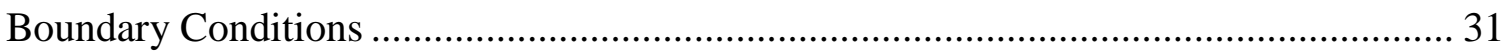

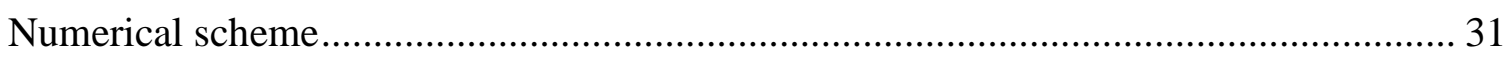

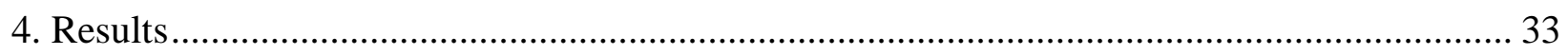

4.1. Experimental Study of Gaseous Combustion ..................................................... 33

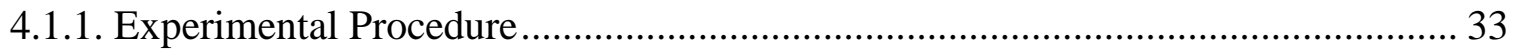

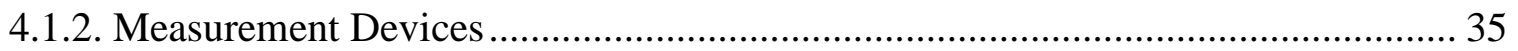

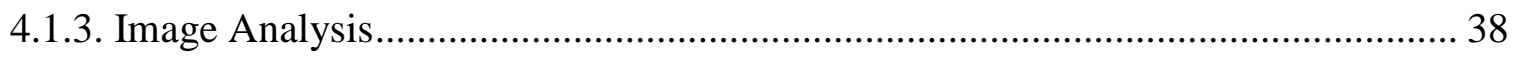

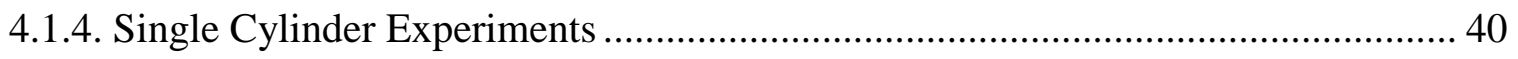

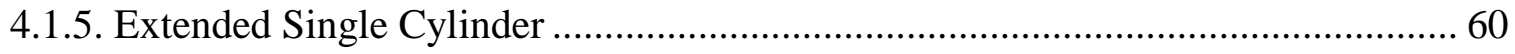

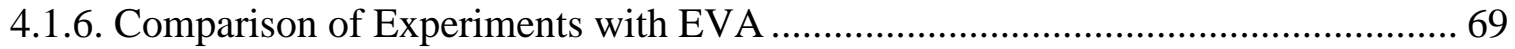


4.2. Effect of Wall Conditions on Flame Acceleration at the Early Stages of Burning in Pipes 75

4.2.1. Impacts of Mechanistic Boundary Conditions.................................................. 76

4.2.2. Impacts of Thermal Boundary Conditions.......................................................... 80

4.3. Analytical Study of a Burning Accident in an Obstructed Coalmining Passage ........... 85

4.3.1. Formulation in 2-D Passage (Channel) …………................................................ 87

4.3.2. Results and Discussion in a 2D Geometry ............................................................ 95

4.3.3. Formulation for the Cylindrical Passage (Tube).................................................. 102

4.3.4. Results and Discussion in a Cylindrical Geometry.............................................. 104

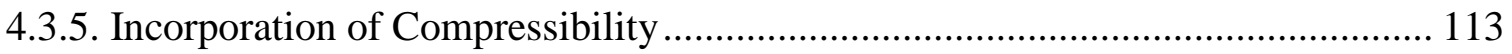

4.3.6. Results and Discussion in a Compressible 2D Geometry................................... 115

4.3.7. Comparison of Theory and Data in the Literature ................................................ 120

4.4. Comparison of Theory with Experiments and Simulations .......................................... 123

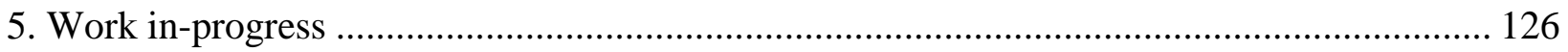

5.1. Dual-Chamber Compartment (Cylinder) …………………………………………..... 126

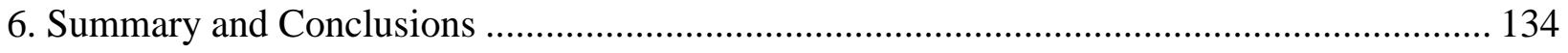

6.1. Experimental Study and Validation of EVA …………............................................. 134

6.2. Numerical Study on Finger Flame Acceleration ...................................................... 135

6.3. Analytical Study towards a Predictive Coal Mining Scenario and Validation with Experiments/Simulations from the Literature............................................................... 136

6.4. Comparison of Acceleration Rates Obtained in Finger Flame Accelerations ............. 137

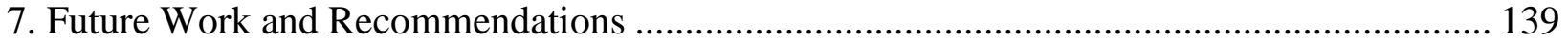

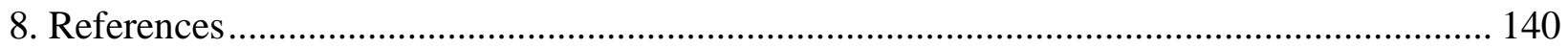




\section{List of Tables}

Table 1: Maximum burning velocities of gaseous combustibles in air at 1 atm [4]................... 4

Table 2: Maximum explosion pressure (gauge) for combustion of premixed gas/air in a closed

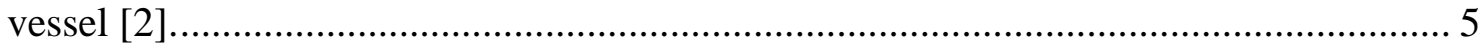

Table 3: Maximum thermal expansion ratio values of some fuel gas/air mixtures [2]............... 7

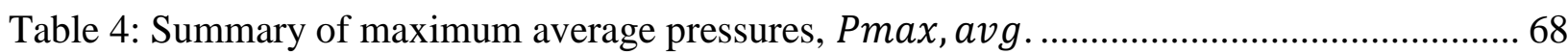

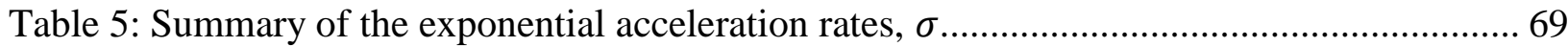

Table 6: The maximal scaled flame tip velocity and the maximal scaled total burning rate for different thermal expansion coefficients. .......................................................... 80 


\section{List of Figures}

Figure 1: Laminar burning velocities FOR methane-, ethane-, propane- and n-heptane-air mixtures versus the equivalence ratio at atmospheric pressure and room temperature [3]........... 3

Figure 2: Premixed gaseous burning at constant pressure with a plane, laminar flame in a one-endopen tube. (a) Ignition at open end of the tube. (b) Ignition at closed end of the tube [2].

Figure 3: Explosion venting in preventing excessively high pressures in the process equipment $[2$, 7]. 10

Figure 4: The experimental setup: rear view: 1) gas analyzer, 2) ignition button, 3) igniter and gas inlet, 4) high-speed camera, 5) computer, 6) mass flow controllers for air and methane.

Figure 5: The experimental setup: front view: 1) transparent cylinder, 2) vent, 3) exhaust and

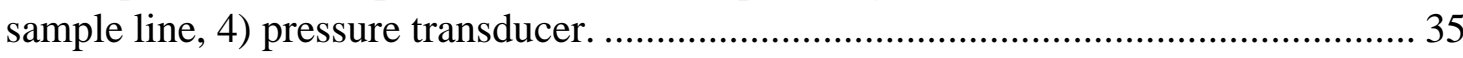

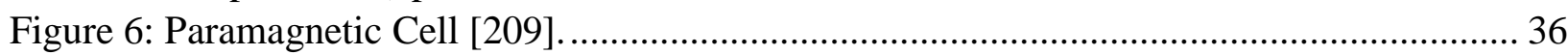

Figure 7: Servomex SERVOTOUGH Oxy 1900 Sample Gas Specifications ................................ 37

Figure 8: PXM409-USBH Pressure Transducer Specifications ………………………………..... 37

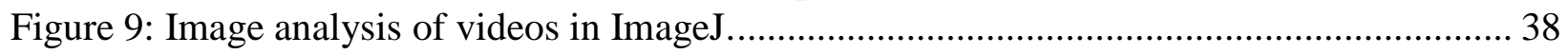

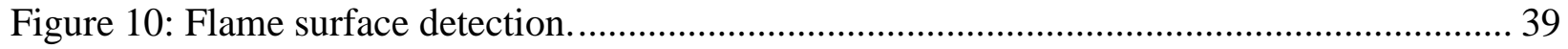

Figure 11: Truncated Circular Cone Method Applied to Flame Surface...................................... 39

Figure 12: Schematic of the single cylinder experimental setup. ................................................ 40

Figure 13: Time evolution of (a) pressure and (b) rate of pressure rise of lean $(\phi=0.8)$, stoichiometric $(\phi=1)$ and rich $(\phi=1.2) \mathrm{CH}_{4}$-air explosions in a single cylinder with small vent and center ignition................................................................................. 41

Figure 14: Time evolution of the flame tip position (a) and velocity (b) towards wall and vent of the cylinder of lean $(\phi=0.8)$, stoichiometric $(\phi=1)$ and rich $(\phi=1.2)$ methane-air explosions in a single cylinder with small vent and center ignition. .......................... 42

Figure 15: The exponential factor $\sigma$ versus the equivalence ratio $\phi$ in a single-cylinder with central ignition and small $\left(67.9 \mathrm{~cm}^{2}\right)$ vent. 43

Figure 16: The time evolution of pressure (a) and of the rate of pressure rise (b) for lean $(\phi=0.8)$, stoichiometric $(\phi=1)$ and rich $(\phi=1.2)$ methane-air burning in a single cylinder with medium vent area $\left(86.6 \mathrm{~cm}^{2}\right)$ and central ignition........................................... 44

Figure 17: Time evolution of the flame tip position (a) and velocity (b) towards wall and vent directions of lean $(\phi=0.8)$, stoichiometric $(\phi=1)$, and rich $(\phi=1.2)$ methane-air flames in a single cylinder with medium vent area $\left(86.6 \mathrm{~cm}^{2}\right)$ and central ignition... 45

Figure 18: Exponential factor $\sigma$ versus equivalence ratio in a single cylinder with central ignition

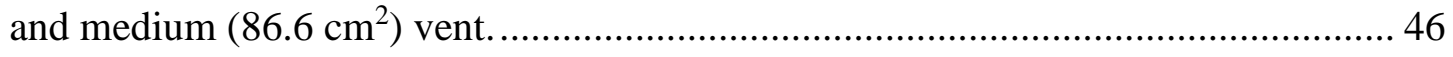

Figure 19: Time evolution of pressure (a) and of the rate of pressure rise (b) of rich $(\phi=1.2)$, stoichiometric $(\phi=1)$ and lean $\phi=0.8$ methane-air explosions in a single cylinder with a large $\left(132.7 \mathrm{~cm}^{2}\right)$ vent area and central ignition.

Figure 20: Time evolution of the flame tip position (a) and velocity (b) towards the wall and the vent for the lean $(\phi=0.8)$, stoichiometric $(\phi=1)$ and rich $(\phi=1.2)$ methane-air 
explosions in a single cylinder with large $\left(132.7 \mathrm{~cm}^{2}\right)$ vent area and central ignition. 48

Figure 21: The exponential factor $\sigma$ versus equivalence ratio $\phi$ in a single cylinder with large $\left(132.7 \mathrm{~cm}^{2}\right)$ vent area and central ignition. 49

Figure 22: Overall scenario of centrally-ignited rich $(\phi=1.2)$ methane-air explosions both inside and outside the cylinder. a) Initial flame expansion inside the cylinder, b) the flame overshoot and 50

Figure 23: Time evolution of pressure (a) and that of the rate of pressure rise (b) for the lean $(\phi=$ $0.8)$, stoichiometric $(\phi=1)$ and rich $(\phi=1.2)$ methane-air explosion in a single cylinder with rear ignition and small $\left(67.9 \mathrm{~cm}^{2}\right)$ vent area.

Figure 24: Time evolution of the flame tip position (a) and velocity (b) for lean $(\phi=0.8)$, stoichiometric $(\phi=1)$ and rich $(\phi=1.2)$ methane-air burnings in a single cylinder with rear ignition and a small vent area $\left(67.9 \mathrm{~cm}^{2}\right)$. 52

Figure 25: Evolutions of the flame surface area (a) and burned gas volume (b) for the lean $(\phi=$ $0.8)$, stoichiometric $(\phi=1)$ and rich $(\phi=1.2)$ methane-air explosions in a single cylinder with a small $\left(67.9 \mathrm{~cm}^{2}\right)$ vent area and rear ignition. 53

Figure 26: Time evolution of pressure (a) and that of the rate of pressure rise (b) for the lean $(\phi=$ $0.8)$, stoichiometric $(\phi=1)$ and rich $(\phi=1.2)$ methane-air explosions in a single cylinder with rear ignition and a medium $\left(86.6 \mathrm{~cm}^{2}\right)$ vent area. 54

Figure 27: Evolutions of the flame tip position (a) and velocity (b) for the lean $(\phi=0.8)$, stoichiometric $(\phi=1)$ and rich $(\phi=1.2)$ methane-air explosions in a single cylinder with a medium $\left(86.6 \mathrm{~cm}^{2}\right)$ vent area and rear ignition............................................ 55

Figure 28: Evolutions of the flame surface area and the burned gas volume for fuel-lean $\phi=0.8$, stoichiometric $\phi=1$ and fuel-rich $\phi=1.2$ methane-air explosions in a single cylinder with medium $\left(86.6 \mathrm{~cm}^{2}\right)$ vent and rear ignition. ..................................................... 56

Figure 29: Time evolution of pressure (a) and that of the rate of pressure rise (b) for the lean $(\phi=$ $0.8)$, stoichiometric $(\phi=1)$ and rich $(\phi=1.2)$ methane-air explosions in a single cylinder with a large vent area $\left(132.7 \mathrm{~cm}^{2}\right)$ and rear ignition................................... 57

Figure 30: Evolutions of the flame tip position (a) and velocity (b) of fuel-lean $\phi=0.8$, stoichiometric $\phi=1$ and fuel-rich $\phi=1.2$ methane-air explosions in a single cylinder with large $\left(132.7 \mathrm{~cm}^{2}\right)$ vent and rear ignition. 58

Figure 31: Evolutions of the flame surface area (a) and of the burnt gas volume (b) for the lean $(\phi=0.8)$, stoichiometric $(\phi=1)$ and rich $(\phi=1.2)$ methane-air explosions in a single cylinder with a large $\left(132.7 \mathrm{~cm}^{2}\right)$ vent and rear ignition................................ 59

Figure 32: The exponential acceleration factor $\sigma$ versus equivalence ratio $\phi$ for methane-air explosions in a single cylinder with rear ignition. 60

Figure 33: Time evolution of pressure (a) and that of the rate of pressure rise (b) for the lean $(\phi=$ $0.8)$, stoichiometric $(\phi=1)$ and rich $(\phi=1.2)$ methane-air explosions in the extended $\left(60 \mathrm{~cm}\right.$ long) single cylinder with a small $\left(67.9 \mathrm{~cm}^{2}\right)$ vent area and rear ignition.

Figure 34: Time evolution of flame tip position (a) and velocity (b) for the lean $(\phi=0.8)$, stoichiometric $(\phi=1)$ and rich $(\phi=1.2)$ methane-air explosions in the extended (60 $\mathrm{cm}$ long) single cylinder with a small $\left(67.9 \mathrm{~cm}^{2}\right)$ vent area and rear ignition. 62 
Figure 35: Time evolution of pressure (a) and of the rate of pressure rise (b) for the lean $(\phi=0.8)$, stoichiometric $(\phi=1)$ and rich $(\phi=1.2)$ methane-air explosions in the extended (60 $\mathrm{cm}$ long) single cylinder with a medium $\left(86.6 \mathrm{~cm}^{2}\right)$ vent area and rear ignition

Figure 36: Evolutions of the flame tip position (a) and velocity (b) for the lean $(\phi=0.8)$, stoichiometric $(\phi=1)$ and rich $(\phi=1.2)$ methane-air explosions in the extended (60 $\mathrm{cm}$ long) single cylinder with medium $\left(86.6 \mathrm{~cm}^{2}\right)$ vent and rear ignition.

Figure 37: The evolution of pressure (a) and that of the rate of pressure raise (b) for the lean $(\phi=$ $0.8)$ and stoichiometric $(\phi=1)$ methane-air explosions in an extended (60 cm long) single cylinder with a large $\left(132.7 \mathrm{~cm}^{2}\right)$ vent area and rear ignition. 65

Figure 38: Evolutions of the flame tip position (a) and velocity (b) for the lean $(\phi=0.8)$ and stoichiometric $(\phi=1)$ methane-air explosions in an extended $(60 \mathrm{~cm}$ long) single cylinder with a large $\left(132.7 \mathrm{~cm}^{2}\right)$ vent area and rear ignition................................ 66

Figure 39: The exponential acceleration rate $\sigma$ versus the equivalence ratio $\phi$ for methane-air explosions in an extended (60 cm long) single cylinder with rear ignition. 67

Figure 40: Comparison of experiment with EVA for stoichiometric methane-air explosion in a single cylinder with small vent $(A v=67.9 \mathrm{~cm} 2)$ and center ignition. 71

Figure 41: Comparison of experiment with EVA for stoichiometric methane-air explosion in a single cylinder with medium vent $(A v=86.6 \mathrm{~cm} 2)$ and center ignition. 71

Figure 42: Comparison of experiment with EVA for stoichiometric methane-air explosion in a single cylinder with large vent $(A v=132.7 \mathrm{~cm} 2)$ and center ignition. 72

Figure 43: Comparison of rear-ignited stoichiometric methane-air explosion experiment with EVA in a single cylinder with small vent $(A v=67.9 \mathrm{~cm} 2)$... 72

Figure 44: Comparison of rear-ignited stoichiometric methane-air explosion experiment with EVA

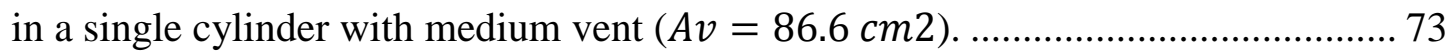

Figure 45: Comparison of rear-ignited stoichiometric methane-air explosion experiment with EVA in a single cylinder with large vent $(A v=132.7 \mathrm{~cm} 2)$. 74

Figure 46: Comparison of peak pressures obtained from the experiments with the EVA. ......... 75

Figure 47: Color temperature snapshots (in K) for the evolution of a flame with $\Theta=8$ in a channel of half-width $H L f=30$ with adiabatic, slip (a) and nonslip (b) walls .................... 77

Figure 48: The scaled flame tip position Ztip/H (a) and the scaled total burning rate $U w / U f$ (b) versus the scaled time $\tau=U f t / H$ for slip (solid) and nonslip (dashed) adiabatic walls for various channel widths. ......................................................................... 78

Figure 49: The scaled flame tip velocity, Utip/Uf, versus the scaled time $\tau=U f t / H$ for the slip (solid) and nonslip (dashed) adiabatic walls with various thermal expansion ratios $\Theta=$ $5,8,10$. 79

Figure 50: The temperature snapshots for the evolution of a flame with the thermal expansion ratio $\Theta=8$ propagating in a channel of half-width $H L f=20$ with the slip, isothermal walls: (a) cold, $T w=298 \mathrm{~K}$ and (b) preheated, $T w=600 \mathrm{~K}$. 81

Figure 51: The scaled total burning rate, $U w / U f$, versus the scaled time $\tau=U f t / H$ for a flame with the thermal expansion ratio $\Theta=8$ propagating in a channel of half-width $H L f=$ 20 with adiabatic walls (solid) and isothermal walls (dashed) at various wall temperatures $T w=298,400,600 \mathrm{~K}$. 82 
Figure 52: The scaled total burning rate, $U w / U f$, versus the scaled time $\tau=U f t / H$ for a flame with the thermal expansion ratio $\Theta=8$ propagating in the channels of half-widths $H L f=10,20,30$ with slip, isothermal cold walls, $T w=298 \mathrm{~K}$. 82

Figure 53: The color temperature snapshots for the evolution of a flame with the thermal expansion ratio $\Theta=10$ in a channel of half-width $H L f=20$ with the slip, isothermal (preheated) walls at $T w=600 \mathrm{~K}$. 83

Figure 54: The scaled total burning rate, $U w / U f$, versus the scaled time $\tau=U f t / H$ for the flames of various thermal expansion ratios $\Theta=5,8,10$ propagating in a channel of half-width $H / L f=20$ with adiabatic walls (a) and preheated isothermal walls with the wall temperatures $T w=400 \mathrm{~K}(\mathrm{~b})$ and $600 \mathrm{~K}(\mathrm{c})$. 84

Figure 55: An illustration of the Bychkov mechanism of flame acceleration in an obstructed passage [206]. 86

Figure 56: Time evolution of the flame tip position Xtip (a) and velocity Utip (b) for the stoichiometric $(\phi=1)$ methane-air mixture with various blockage ratios $\alpha=$ $0,1 / 3,1 / 2,2 / 3(n=1.4)$. 97

Figure 57: Time evolution of the flame tip position Xtip (a) and velocity Utip (b) in 2D geometry for stoichiometric $\mathrm{CH}_{4}$-air burning with various blockage ratios $\alpha=0,1 / 3,1 / 2,2 / 3$ and various power factors $n=1.33,1.4,1.5$. 97

Figure 58: Time evolution of the flame tip position Xtip (a) and velocity Utip (b) for the lean $(\phi=0.8)$, stoichiometric $(\phi=1)$ and rich $(\phi=1.2)$ methane-air mixtures with various blockage ratios: $\alpha=0,1 / 3,1 / 2,2 / 3$..... 98

Figure 59: Time evolution of the flame tip position Xtip (a) and velocity Utip (b) in a $2 \mathrm{D}$ geometry for highly-lean $(\phi=0.6)$ and highly-rich $(\phi=1.4) \mathrm{CH}_{4}$-air burning with various blockage ratios $\alpha=0,1 / 3,1 / 2,2 / 3$. 99

Figure 60: Time evolution of the flame tip position Xtip (a) and velocity Utip (b) in a 2D geometry for lean $\mathrm{CH}_{4}$-air burning of $\phi=0.7$, without and with dust particles (combustible, inert, and combined) of particle radius $r s=75 \mu \mathrm{m}$ and concentration $c s=50 \mathrm{gm} 3$, for various blockage ratios: $\alpha=0,1 / 3,2 / 3$. 99

Figure 61: Time evolution of the flame tip Xtip (a) and velocity Utip (b) for a lean methane airmixture of $\phi=0.7$ with and without dust particles (inert, combustible, and combined) of radius $r s=75 \mu \mathrm{m}$ and concentration $c s=120 \mathrm{~g} / \mathrm{m} 3$, for the blockage ratios: $\alpha=$ $0,1 / 3,1 / 2,2 / 3$.

Figure 62: Time evolution of the flame tip position Xtip (a) and velocity Utip (b) in a 2D geometry for lean $\mathrm{CH}_{4}$-air burning of $\phi=0.7$, without and with dust particles (combustible, inert, and combined) of particle radius $r s=75 \mu \mathrm{m}$ and concentration $c s=250 \mathrm{gm} 3$, for various blockage ratios: $\alpha=0,13,23$. 101

Figure 63: Time evolution of the flame tip Xtip (a) and velocity Utip (b) for a lean methane airmixture of $\phi=0.7$ with and without dust particles (inert, combustible, and combined) of radius $r s=10 \mu \mathrm{m}$ and concentration $c s=120 \mathrm{~g} / \mathrm{m} 3$, for the blockage ratios: $\alpha=$ $0,1 / 3,1 / 2,2 / 3$. 101

Figure 64: Evolution of the flame tip position Ztip (a) and velocity Utip (b) for the stoichiometric $(\phi=1)$ methane-air mixture with various blockage ratios $\alpha=0,1 / 3,1 / 2,2 / 3 \ldots 106$ 
Figure 65: Time evolution of the flame tip position Ztip (a) and velocity Utip (b) in a cylindricalaxisymmetric geometry for stoichiometric $\mathrm{CH}_{4}$-air burning with various blockage ratios $\alpha=0,1 / 3,1 / 2,2 / 3$ and various power factors $n=1.33,1.4,1.5$. 107

Figure 66: Evolution of the flame tip position Ztip (a) and velocity Utip (b) for the lean $(\phi=$ $0.8)$, stoichiometric $(\phi=1)$, and rich $(\phi=1.2)$ methane-air mixtures with various $\alpha=0,1 / 3,1 / 2,2 / 3$. 108

Figure 67: Time evolution of the flame tip position Ztip (a) and velocity Utip (b) in a cylindricalaxisymmetric geometry for highly-lean $(\phi=0.6)$ and highly-rich $(\phi=1.4) \mathrm{CH}_{4}$-air burning with various blockage ratios $\alpha=0,1 / 3,1 / 2,2 / 3$. 108

Figure 68: The flame run-up distance versus the equivalence ratio $\phi$ of a methane-air mixture at various blockage ratios: $\alpha=0,1 / 3,1 / 2,2 / 3$. 109

Figure 69: Time evolution of the flame tip position Ztip (a) and velocity Utip (b) in a cylindricalaxisymmetric geometry for lean $\mathrm{CH}_{4}$-air burning of $\phi=0.7$, without and with dust particles (combustible, inert, and combined) of particle radius $r s=75 \mu \mathrm{m}$ and concentration $c s=50 \mathrm{gm} 3$, for various blockage ratios: $\alpha=0,1 / 3,2 / 3$. 110

Figure 70: Evolution of the flame tip position Ztip (a) and velocity Utip (b) for a lean methaneair mixture of $\phi=0.7$ with and without dust particles (inert, combustible, and combined) of radius $r s=75 \mu \mathrm{m}$ and concentration $c s=120 \mathrm{~g} / \mathrm{m} 3$, for various blockage ratios: $\alpha=0,1 / 3,2 / 3$.

Figure 71: Time evolution of the flame tip position Ztip (a) and velocity Utip (b) in a cylindricalaxisymmetric geometry for lean $\mathrm{CH}_{4}$-air burning of $\phi=0.7$, without and with dust particles (combustible, inert, and combined) of particle radius $r s=75 \mu \mathrm{m}$ and concentration $c s=250 \mathrm{gm} 3$, for various blockage ratios: $\alpha=0,1 / 3,2 / 3$.

Figure 72: Evolution of the flame tip position Ztip (a) and velocity Utip (b) for a lean methaneair mixture of $\phi=0.7$ with and without dust particles (inert, combustible, and combined) of radius $r s=10 \mu \mathrm{m}$ and concentration $c s=120 \mathrm{~g} / \mathrm{m} 3$, for various blockage ratios: $\alpha=0,1 / 3,2 / 3$. 112

Figure 73: Evolution of flame tip position, Ztip (a), and velocity, Utip (b), for stoichiometric $\mathrm{CH}_{4}$ air burning with various mining blockage ratios considered: $\alpha=0,1 / 3,1 / 2,2 / 3.116$

Figure 74: Evolution of flame tip position, Ztip, (a) and velocity, Utip, (b) of a $\mathrm{CH}_{4}$-air flame at various equivalence ratios: $\phi=0.8 ; 1$ and 1.2, with a fixed blockage ratio of $\alpha=1 / 3$.

Figure 75: Evolution of the flame tip position Ztip in passages of blockage ratios $\alpha=0$ and 1/3 for lean $(\phi=0.8)$, stoichiometric $(\phi=1)$ and rich $(\phi=1.2) \mathrm{CH}_{4}$-air burning accounting for gas compression.

Figure 76: Evolution of flame tip position, Ztip, (a) and velocity, Utip, (b) for lean $(\phi=0.7)$ $\mathrm{CH}_{4}$-air burning, with and without dust particles (inert, combustible, and combined) of radius $r s=75 \mu \mathrm{m}$ and concentration $c d=120 \mathrm{~g} / \mathrm{m} 3$ in a passage with a blockage ratio of $\alpha=1 / 3$.

Figure 77: Evolution of flame tip position, Ztip, (a) and velocity, Utip, (b) for lean $(\phi=0.7)$ methane-air burning, with and without dust particles (inert, combustible, and 
combined) of radius $r s=10 \mu \mathrm{m}$ and concentration $c d=120 \mathrm{~g} / \mathrm{m} 3$ in a passage with a blockage ratio of $\alpha=1 / 3$.

Figure 78: Comparison of theoretical formulation with the experiments [232] and numerical simulations [208] (FAST, yellow, \& ALLA, blue): the flame tip velocity vs its position for stoichiometric methane explosion in in obstructed channel with height $H=$ $0.174 \mathrm{~m}$ and blockage ratios $\alpha=0.3$ (a) \& 0.6 (b).

Figure 79: Comparison of theoretical formulation with the experiments [232] and numerical simulations [208] (FAST, yellow, \& ALLA, blue): the flame tip velocity vs its position for stoichiometric methane explosion in in obstructed channel with height $H=0.52 \mathrm{~m}$ and blockage ratios $\alpha=0.3$ (a) \& 0.6 (b). 122

Figure 80: Comparison of acceleration rates obtained from the experiments (markers) and calculated from the theory (solid lines) for centrally-ignited fuel-lean, stoichiometric and fuel-rich methane-air explosions in a single cylinder with height of $H=19 \mathrm{~cm}$ and length of $L=30 \mathrm{~cm}$. 124

Figure 81: Comparison of acceleration rates obtained from the experiments (markers) and calculated from the theory (solid lines) for rear-ignited fuel-lean, stoichiometric and fuel-rich methane-air explosions in a single cylinder with height of $H=19 \mathrm{~cm}$ and length of $L=60 \mathrm{~cm}$.

Figure 82: Comparison of acceleration rates obtained from the numerical simulations (markers) and calculated from the theory (solid lines) for rear-ignited methane-air explosions in a channel with various heights. 125

Figure 83: The experimental setup used for the dual-chamber compartment experiments........ 126

Figure 84: Pressure evolution for the lean $(\phi=0.8)$ methane-air explosions in a dual-chamber compartment cylinder with a medium $\left(86.6 \mathrm{~cm}^{2}\right)$ vent: a) Test 1, b) Test 2 , c) Test 3 . 127

Figure 85: Evolutions of the flame tip position (a) and velocity (b) for the lean $(\phi=0.8)$ methaneair explosion in a dual-chamber compartment cylinder with medium $\left(86.6 \mathrm{~cm}^{2}\right)$ vent and rear ignition. 128

Figure 86: Pressure evolution for the stoichiometric $(\phi=1)$ methane-air explosions in a dualchamber compartment cylinder with a medium $\left(86.6 \mathrm{~cm}^{2}\right)$ vent and rear ignition: a) Test 1, b) Test 2, c) Test 3 .

Figure 87: The evolutions of the flame tip position (a) and velocity (b) for stoichiometric $\phi=1$ methane-air explosions in dual-chamber compartment cylinder with medium (86.6 $\mathrm{cm}^{2}$ ) vent and rear ignition.

Figure 88: The pressure evolution of three repeated rich $(\phi=1.2)$ methane-air explosion experiments in dual-chamber compartment cylinder with medium $\left(86.6 \mathrm{~cm}^{2}\right)$ vent and rear ignition: a) Test 1, b) Test 2, c) Test 3 . 131

Figure 89: Evolutions of the flame tip position (a) and velocity (b) for the rich $(\phi=1.2)$ methaneair explosion in a dual-chamber compartment cylinder with a medium $\left(86.6 \mathrm{~cm}^{2}\right)$ vent area and rear ignition. 132 


\section{Nomenclature}

$R_{f}$ : radius of a globally-spherical expanding flame front

$C$ : constant defined as $C=k_{D L}^{n-1}\left(\Theta U_{f} / n\right)^{n}$

$t$ : time

$n$ : Darrieus-Landau instability exponent

$k_{D L}:$ Darrieus-Landau cutoff wavenumber

$U_{f}$ : laminar flame velocity

$\rho_{\text {fuel }}:$ density of fuel mixture

$\rho_{\text {burnt }}:$ density of burnt gas

$L_{f}:$ flame thickness

$D_{t h}:$ thermal diffusivity coefficient

$U_{D L}$ : instantaneous radial flame velocity

$H$ : half-width of a two-dimensional (2D) passage

$R$ : radius of a cylindrical passage

$\Delta z:$ obstacle spacing

$x$, : radial direction

$z:$ axial direction

$R_{f}(t):$ flame "skirt"

$\Delta z:$ obstacle spacing

$u_{x}, u_{r}:$ radial velocity

$u_{z}:$ axial velocity

$Z_{\text {tip }}:$ flame tip position 
$U_{\text {tip }}$ : flame tip velocity

$t_{o b s}:$ the time flame skirt touches an obstacle

$R_{f}\left(t_{o b s}\right)$ : flame "skirt" radius at $t_{o b s}$

$Z_{t i p}\left(t_{o b s}\right):$ flame tip position at $t_{o b s}$

$U_{D L}\left(t_{o b s}\right):$ global flame velocity at $t_{o b s}$

$t_{\text {rud }}:$ flame run-up time

$c_{0}:$ speed of sound

$Z_{\text {rud }}$ : flame run-up distance

$R_{f, o}$ : flame "skirt" in Ref. [209]

$t_{f}(z)$ : the instant at which the fresh gas between obstacles at the position $z$ starts burning

$U_{d, f}$ : laminar flame velocity in an "effective" gaseous-dusty environment

$C_{p}:$ specific heat of gaseous air-fuel mixture

$C_{T}$ : entire specific heat

$T_{f}$ : flame temperature with particles

$T_{b}$ : adiabatic flame temperature based on purely methane-air equivalence ratio

$T_{u}:$ unburnt gas temperature

$E$ : activation energy

$R_{u}$ : gas constant

$M$ : molar masses

$m:$ original masses

$C_{s}:$ specific heat of dust particles

$n_{s}:$ number of particles per unit volume

$V_{S}$ : volume of a single particle 
$\rho_{s}:$ density of dust

$\rho$ : density of gaseous-dusty fuel-air mixture

$\rho_{u}:$ density of gas

$c_{S}:$ concentration of the particles

$r_{s}:$ radius of a particle

$Q$ : volumetric heat release

$L_{v}$ : heat of gasification per unit volume

$n_{\text {air }}$ : number of moles of air per unit volume

$V_{\mathrm{CH}_{4}}$ : volume of methane

$V_{\text {air }}$ : volume of air

$n_{\text {product }}:$ number of moles of the burning products

\section{Greek letters}

$\alpha$ : blockage ratio

$\phi$ : equivalence ratio

$\lambda_{D L}$ : Darrieus-Landau critical wavelength

$\Theta \equiv \rho_{u} / \rho_{b}:$ thermal expansion ratio

$\beta$ : defined as $\sqrt{\Theta(\Theta-1)}$

$\phi_{s}$ : modified equivalence ratio in the gaseous-dusty air mixture

\section{Subscripts}

$f$ : flame

$D L$ : Darrieus-Landau

fuel : fuel mixture 


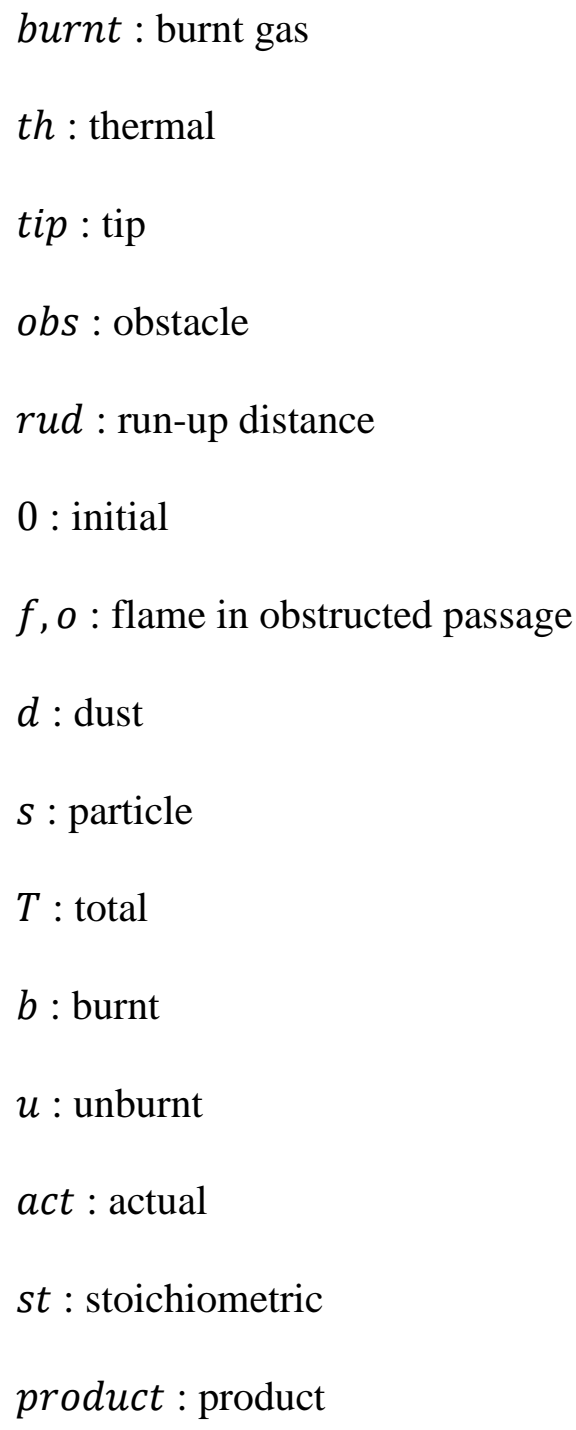




\section{Introduction: Combustion Science - the State of the Art}

Combustion science is a well-understood topic, at least in the laminar regimes of burning. Its applications are known in daily life. Propane, for example, is often used for lightning, heating and cooking, if electricity is not easily available. In spite of technological developments in nuclear, solar, wind and hydroelectric energetics, burning of fossil fuels still supplies the major fraction of the total energy need of the world and the trajectory shows that this trend will continue in the near future [1].

Combustion energy is mainly used to generate heat and power. The design and operation of heat and power devices and engines are closely related to the efficient utilization of combustion, which also has an impact on pollution and health. Such outcomes of the combustion process as $\mathrm{NO}_{\mathrm{x}}$, for example, forms smog when it reacts with volatile organic compounds in the presence of sunlight. A more serious problem, carrying a catastrophic potential, is global warming due to the increased amount of anthropogenic carbon dioxide $\left(\mathrm{CO}_{2}\right)$ in the atmosphere. Incineration is still being used for disposing toxic wastes. Safety is also a primary concern in mine galleries, onshore and offshore process industries, and society.

The term "explosion" usually evokes a destructive image to most. However, most of the explosions are not actually destructive, but planned and controlled. The internal combustion engines are the most apparent examples. Every minute, millions of explosions are undoubtedly happening in the world to provide power in transportation. Explosions have also been used in blasting in quarries and other earthmoving processes, i.e. road, tunnel, or dam constructions. Though major explosions are controlled, accidental ones, which result in injury, death and destruction are of great concern.

Two accidental explosion categories account for the major accidental explosions encountered in the process industries, namely, gas/vapor explosion and dust explosions. Both types of explosions have similar ignition and combustion characteristics such as flammability/explosibility limits, laminar burning velocities and quenching distances, detonation onsets, well-defined minimum ignition energies and adiabatic constant-volume explosion pressures of similar magnitude. One basic difference is that gas/vapor explosions occur only when the fuel/air ratio is between the flammability limits. In the case of settled dust layers, the settle dusts are porous in contrary to the combustible gases/vapors. As a result, they contain air in the voids between particles, which makes 
it possible for a sustained combustion in such layers. The fundamental difference is that in the clouds of dust particles, the fuel particles are generally larger than the air molecules such that their movement is controlled by inertial forces, including gravity. On the other hand, once a combustible gas is mixed, homogeneously, with air, the resulting mixture tends to stay homogeneous due to random molecular motion [2]. This dissertation deals only with accidental gas explosions.

When discussing combustion phenomena, it is important to distinguish premixed and nonpremixed combustion. In non-premixed combustion, burning is controlled by diffusion of fuel and oxidizer. The fuel and the oxidizer are initially separated, so the reaction occurs only at an interface where the fuel makes contact with the oxidizer. This interface is also called reaction zone. In gas explosions, the situation is different. In this case, the fuel and the oxidizer are mixed at a molecular level. The combustion starts once initiated and readily propagates throughout the unburnt mixture. This kind of combustion is called premixed combustion.

\subsection{Laminar Burning Velocity of Premixed Gas/Vapor and Air}

Under "normal conditions", i.e. room temperature and atmospheric pressure, the rate of chemical reactions in most combustible mixtures is negligible. A mixture of gas/vapor with oxidizer ignites only when there is a spark, which can heat the local mixture volume, or another heat source is supplied. Then, after ignition, a reaction wave starts moving through the mixture.

Under a subsonic regime, a self-sustained wave of the exothermic chemical reaction that spreads through a homogeneous combustible mixture is called the deflagration (premixed flame). At this regime, the propagation of the chemical reaction wave is associated with molecular diffusion and thermal conductivity. As the flame propagates, the heat generated in the chemical reaction is conducted to neighboring unburned mixture, heats this mixture and initiates the chemical reaction in the neighboring mixtures. The burning process is typically active in a very thin layer that is called a reaction zone or a flame thickness. Comparing to the length scale of the problem, this region can be considered as a smooth, planar sheet traveling at a constant velocity through the quiescent unburned gas/air mixture. This velocity is called the laminar burning velocity.

Figure 1 presents the experimentally determined laminar burning velocities expressed versus the equivalence ratio, for four common combustible gases burning in air. It illustrates that for any combustible gas, the laminar burning velocity has its maximum value when the fuel/air ratio is 
close to stoichiometry. At stoichiometric condition, the fuel and the oxidizer are mixed in an ideal ratio that all the oxygen available transforms all the fuel to $\mathrm{CO}_{2}$ and $\mathrm{H}_{2} \mathrm{O}$. Any deviations from the stoichiometry leads to lower laminar burning velocity value, whether in the direction of fuel-rich or fuel-lean mixtures. For alkanes, the maximum laminar burning velocity values do not change much, as shown in Fig. 1, and fall in the range $40 \sim 50 \mathrm{~cm} / \mathrm{s}[3]$.

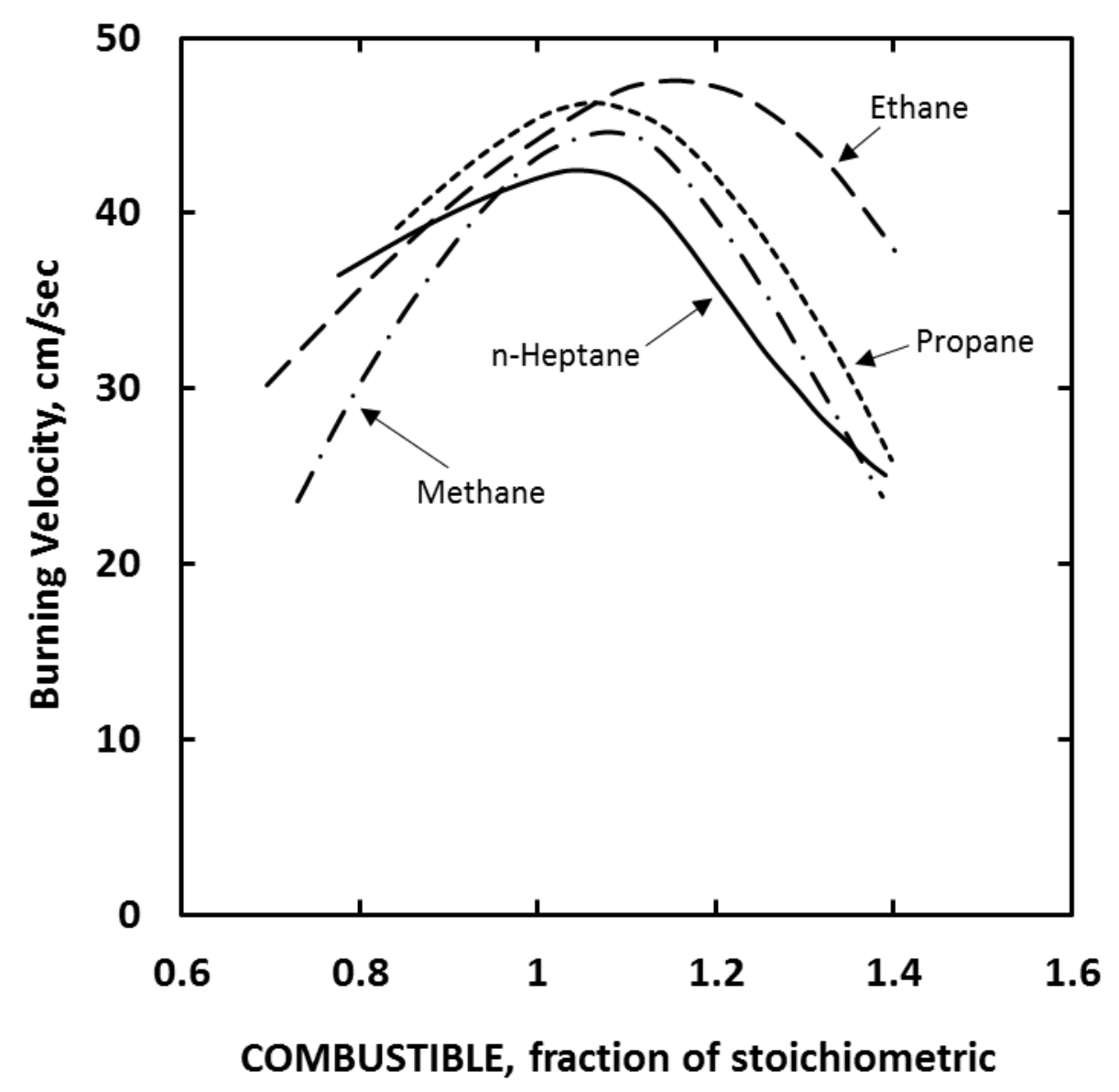

Figure 1: Laminar burning velocities FOR methane-, ethane-, propane- and n-heptane-air mixtures versus the equivalence ratio at atmospheric pressure and room temperature [3].

Table 1 presents the maximum experimental laminar burning velocity values for some combustible gases [4]. Unsaturated hydrocarbons, i.e. acetylene, hydrogen, ethylene and ethylene oxide, can have considerably higher laminar burning velocities as compared to the paraffins and most of their derivatives. 
Table 1. Maximum burning velocities of gaseous combustibles in air at 1 atm [4].

\begin{tabular}{|cc|}
\hline Combustibles & Maximum Laminar Burning Velocity $(\mathrm{cm} / \mathrm{s})$ \\
\hline Alkanes (including methane) & $40-50$ \\
Natural gas & 38.5 \\
Acetone & 42.5 \\
Benzene & 48 \\
Propylene & 51 \\
Methanol & 57 \\
Propylene oxide & 67 \\
Ethylene & 74.5 \\
Ethylene oxide & 89.5 \\
Acetylene & 157 \\
Hydrogen & 326 \\
\hline
\end{tabular}

\subsection{Maximum Pressure Generated from Combustion of Premixed Gas/Vapor and Air at Constant Volume}

When combustion starts at constant volume, pressure will rise. Approximately, the adiabatic flame temperature can be determined from the equation

$$
T_{2}-T_{1}=\Delta E / C_{v},
$$

where $\Delta E$ is the heat generated from combustion per mole at constant volume, $C_{v}$ is the mean specific heat of the gaseous mixture at constant volume (averaged over the respective temperature range), $T_{1}$ is the initial temperature of the fuel mixture (before combustion) and $T_{2}$ is the final temperature of the burning products after complete combustion. Once the final temperature is obtained and assuming that the gases/vapors are ideal, the pressure rise resulting from the constantvolume adiabatic combustion can be calculated using the ideal gas relation

$$
P_{2} / P_{1}=n_{2} T_{2} / n_{1} T_{1} \text {. }
$$

Here $P$ is the pressure (absolute), $n$ the total number of moles of gas in the constant volume and $T$ the temperatures $(\mathrm{K})$. Experimental values of $P_{2}$ for some combustible gases are given in Table 2 $[2]$. 
Table 2: Maximum explosion pressure (gauge) for combustion of premixed gas/air in a closed vessel [2].

\begin{tabular}{|cc|}
\hline Fuel & Maximum Pressure, bar $(\mathrm{g})$ \\
\hline Acetylene & 10.3 \\
Ethylene oxide & 9.9 \\
Acetone & 8.9 \\
Ethylene & 8.9 \\
n-Heptane & 8.6 \\
Propane & 8.6 \\
Ethyl alcohol & 7.5 \\
Hydrogen & 7.4 \\
Carbon monoxide & 7.3 \\
Methane & 7.2 \\
\hline
\end{tabular}

Maximum pressures given in Table 2 were obtained at normal atmospheric initial pressure. Different initial temperature and pressure results in correspondingly different $P_{2}$ values. As a rule, the maximum pressure generated in a constant volume adiabatic combustion is proportional to the initial pressure prior to combustion [2]. For example, a mixture that produces 8 bar (abs.) final pressure at 1 bar (abs.) initial pressure will result in 16 bar (abs.) final pressure at 2 bar (abs.) initial pressure. In the case of gas explosions in a series of closed volumes, coupled with a duct or pipe, an explosion in one part of the system will promote the pressure to considerably high values while in the other part the fresh fuel/air mixture still remains. When the remaining mixture starts burning, very high pressure transients can be attained despite the whole system was initially at atmospheric pressure.

\subsection{Thermal Expansion Ratio of Premixed Gas/Vapor and Air}

The thermal expansion coefficient is one of the most important flame parameters. During combustion, the gas is heated and it expands, and because of such expansion, the burning process becomes strongly coupled to the gaseous flow [5]. Figure 2 illustrates idealized adiabatic, smoothwall (no interference of the wall with the flow), no buoyancy, planar, laminar combustion of quiescent premixed combustible gas/air in a one-end-open straight tube/duct, at constant pressure. When the mixture is ignited at an open end of the tube, the combustion products will expand freely 
into the environment, whereas still fresh fuel/air mixture remains quiescent. The observed flame speed towards the unburnt gas will be identical with the actual laminar burning velocity of gas/air mixture. If ignition occurs in the closed end of the tube, on the other hand, the expansion of the combustion products forces the flame towards the same direction of flame propagation. Therefore, the unburnt gas will be pushed towards the open end. The observed flame speed, in this case, becomes the sum of the velocity of the unburnt gas flow and the laminar burning velocity as

$$
U_{f e}=U_{f}+U_{g},
$$

and

$$
U_{f e}=U_{f} \cdot \Theta .
$$

Equations (1.3) and (1.4) describe how the generated heat from combustion gives rise to expansion. Here, $\Theta$ is the thermal expansion ratio defined as $\left(1+U_{g} / U_{f}\right)$ in Eq. (1.3) or $U_{f e} / U_{f}$ in Eq. (1.4).

(a)

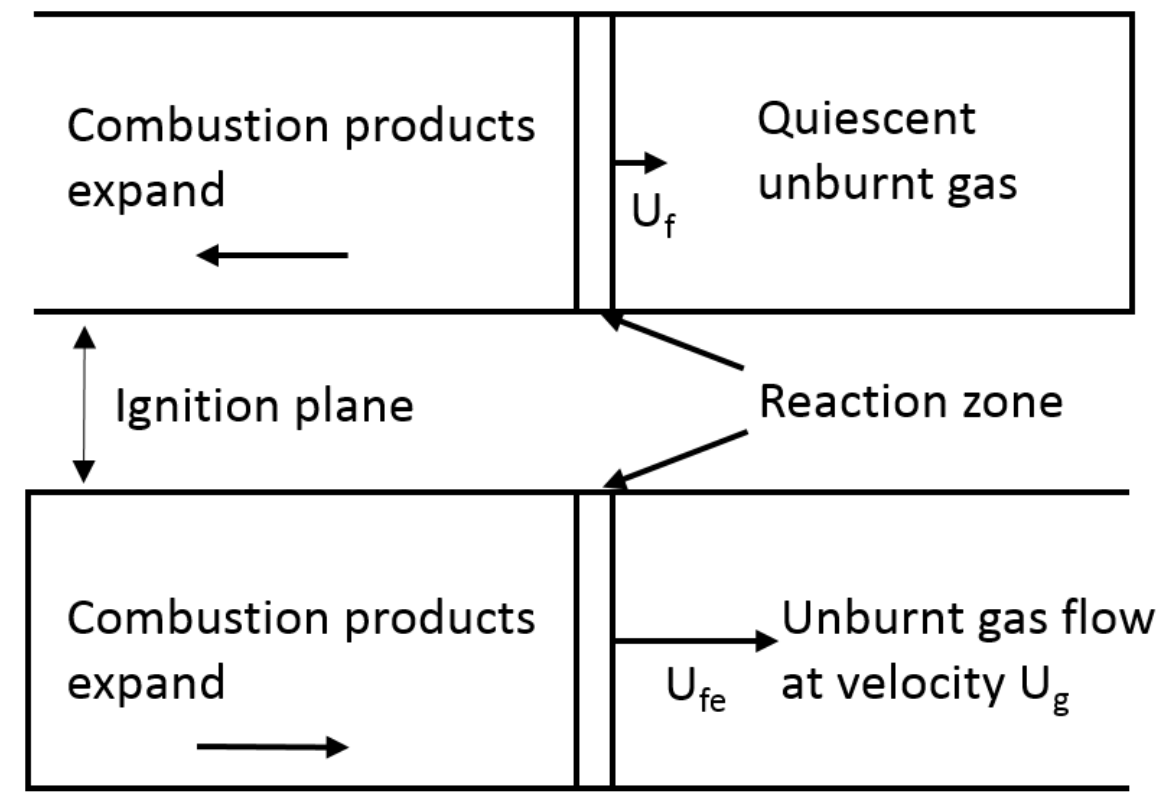

Figure 2: Premixed gaseous burning at constant pressure with a plane, laminar flame in a one-end-open tube. (a) Ignition at open end of the tube. (b) Ignition at closed end of the tube [2].

In other words, the thermal expansion ratio $\Theta$ is the ratio of the volume that a quantity of a premixed gas has attained after complete, adiabatic ideal constant pressure combustion, to its initial volume before combustion. The value $\Theta$ varies with the equivalence ratio $\phi$ for a given mixture, and it has maximum values near stoichiometric condition or somewhat higher. Table 3 presents the maximum (stoichiometric) values of thermal expansion ratios for some hydrocarbons. 
Table 3. Maximum thermal expansion ratio values of some fuel gas/air mixtures [2].

\begin{tabular}{|cc|}
\hline Fuel & Thermal Expansion Ratio $\Theta$ \\
\hline Acetylene & 8.38 \\
Butane & 8.06 \\
Propane & 7.98 \\
Ethane & 7.87 \\
Ethylene & 7.48 \\
Methane & 7.25 \\
Hydrogen & 6.88 \\
\hline
\end{tabular}

The reason behind thermal expansion is the large amount of heat liberated from the combustion reaction of fuel and oxygen. The burning velocity is the velocity at which the flame "consumes" the fresh unburnt gas/air mixture, whereas the flame speed denotes the relative speed of the flame front towards the unburnt mixture to a stagnant observer. If combustion occurs under a condition where the burnt gas cannot expand freely, the heat generated by the reaction may give a significant rise, which may result in a substantial damage to process equipment, plants, buildings.

\subsection{The Darrieus-Landau Instability of a Thin Flame Front}

A flame front seldom has planar shape but usually acquires a curved shape. In the laminar regime, the curved shape of a flame requires an explanation. A flame front may become spontaneously curved due to the hydrodynamic instability discovered by Darrieus and Landau (DL instability). This can be explained by a linear stability analysis of a small initial perturbation of the front. If the amplitude increases, the front is unstable, and if the amplitude decreases, the front is stable. Darrieus and Landau treated the flame front as a surface of zero thickness separating the fresh fuel and the burnt matter and propagating towards the fuel with a constant velocity $U_{f}$. Perturbations of a planar front is in the form

$$
\tilde{F}(x, t)=\tilde{F}(x) \exp (\sigma t+i k y),
$$

where $k=2 \pi / \lambda$ is the perturbation wave-number, $\lambda$ is the wavelength of the perturbation, $\sigma$ is the perturbation growth rate. The idea is to find the dispersion relation that is the growth rate as a function of the wave number $\sigma=\sigma(k)$ [5]. If $\operatorname{Re}(\sigma)$ is positive, the front is unstable and the amplitude of wrinkling grows exponentially with time. If $\operatorname{Re}(\sigma)$ is negative, the front is stable and 
the amplitude of wrinkling decreases exponentially with time. The amplitude of the perturbation is governed by a second-order differential equation [6]

$$
\left(1+\frac{\rho_{b}}{\rho_{u}}\right) \frac{d^{2} \tilde{F}}{d t^{2}}+2\left(U_{f} k\right) \frac{d \tilde{F}}{d t}-\left(\frac{\rho_{u}}{\rho_{b}}-1\right)\left(U_{f} k\right)^{2} \tilde{F}=0,
$$

where $\rho_{u}$ and $\rho_{b}$ are the densities of unburnt and burnt mixtures, respectively. Substituting Eq. (1.5) and introducing $\Theta=\rho_{u} / \rho_{b}$ yield the following relation for $\sigma$

$$
\sigma=k U_{f} \frac{\Theta}{\Theta+1}\{ \pm \sqrt{\Theta+1-1 / \Theta}-1\} .
$$

Equation (1.7) is called the dispersion relation for the DL instability for infinitely thin flame front. The existence of the positive root indicates that the flame front is unstable against perturbations of any wave length. The relation shows that this instability is universal and is due to the gas expansion across the flame front. If there was no gas expansion, i.e. $\Theta=1$, there would be no instability. However, this is not the case for real flames.

\subsection{Safety in Industry - a Persistent Challenge}

"Learning by doing" has been an effective method of acquiring new knowledge. This holds true, in explosions, too - unfortunately, in the expense of many human lives and suffering, as well as material damage and loss of profit as experienced in history. The biggest challenge in achieving safety in industry is to establish a high safety level and, once established, to actively secure such levels by continuous maintenance and renewal. Those who have experienced an explosion accident have a profound appreciation of the realism of this hazard. However, those who only read or heard about such accidents often do not have the real understanding of risks of accidental explosions. The motivation is, therefore, to acquire full understanding of accidental explosions for minimizing the probability of such accidents in the future and to let this new knowledge lead to less dramatic consequences.

Education plays a key role in the process safety, which includes the topics of reliability and risk analysis; the physics, chemistry and technology of processes and hazards; and means of accident prevention and mitigation. A considerable emphasis has been focused on the methods of reliability and risk analysis to improve the process safety. However, education in physics, chemistry and technology of processes and process hazards have been left somewhat behind. 


\section{Literature Survey}

\subsection{Vented Gas Explosion}

Explosion risk can be defined as the probability of an explosion and its expected consequences [2]. Therefore, it is sought to minimize either of the explosion probability or explosion consequences, or both. In gas explosion risk management, the following aims have higher priority:

- Preventing gas leaks from the process equipment.

- Minimizing size of gas cloud in case of accidental leaks by early leak detection.

- Preventing ignition sources.

- Controlling ignition sources.

- Mitigating/controlling gas/vapor cloud explosions once initiated despite preventive measures.

Preventing and controlling of ignition sources may sound similar. However, "preventing" means removal of any ignition sources, while "controlling" refers to the use of any ignition sources such as an open flame provided that no explosive gas/vapor cloud can exist in the environment. Despite all four initial preventive measures, means of mitigating/controlling gas/vapor cloud explosions are also essential for preventing accidental explosions from their catastrophic consequences. One of the mitigating techniques is the use of explosion venting. This process is illustrated in Fig. 3. The maximum explosion pressure inside the process equipment is basically a result of two competitors:

- Burning process of the explosive cloud, thereby generating heat and increasing the pressure, - Venting process of burnt, unburnt and burning cloud through the vent, relieving the pressure. Explosion venting has its popularity because it is a simple and yet effective method. During a possible explosion, a venting device serves as a failing part of the process equipment under a relatively low pressure allowing for controlled discharge of burnt, unburnt and burning gases into environment. By doing so, excessively high pressures that develops in totally closed vessel which may lead to total equipment failure are prevented. 

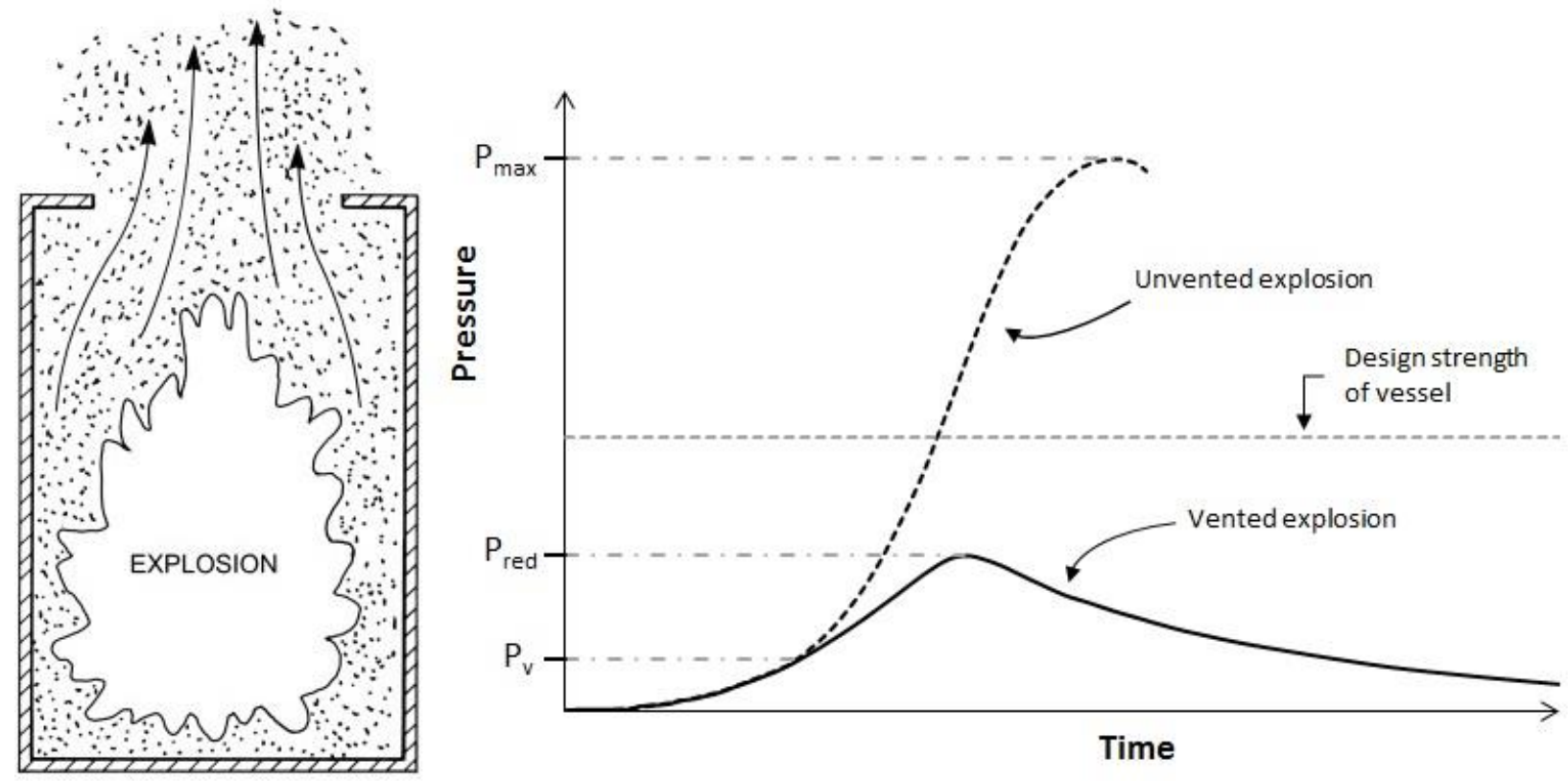

Figure 3: Explosion venting in preventing excessively high pressures in the process equipment $[2,7]$.

Work on vented gas explosions dates back as early as to 1933 when a systematic investigation was conducted at the Pittsburgh Experiment Station of the US Bureau of Mines in cooperation with the Western Electric Co., Inc. on the protection of cellulose acetate wire-coating machines involving explosive acetone-air mixtures during the manufacturing process [8, 9]. Jones et al. [9] provided a relation of pressures developed to area of release opening for acetone-air concentration producing the maximum pressure in a laboratory bomb and they applied this relation to the commercial-size cellulose acetate wire-coating machine to determine adequate diaphragm releases preventing the pressure from rising above a safe value. In no case, the determined pressure by experiment was higher than the calculated one. Murphy [10] yielded a formula to calculate the area of rupture disk for protecting equipment from overpressure, provided the necessary relieving capacity was known. Cousins \& Cotton [11] provided data on hydrogen- and propane-air explosions in four different test vessels: pipe, drum, tank, and a high-strength vessel. Specifically, they investigated the effects of the length-to-diameter $(L / D)$ ratio (the aspect ratio), the vent size, rupture disk strength and initial pressure on maximum explosion pressure. Lamb [12] described the relief valves as possible remedy to prevent personnel injury and property damage because of explosions in reciprocatingengine crankcases. Requirements of relief valves were given such that the gases be released at a rate to keep the pressure within safe working pressure, and also that the opening of relief valve be instantly closed immediately after the excess pressure had been released to prevent the entry of air 
into the crankcase, and a secondary explosion. Making the released hot gases non-injurious to personnel and incapable of starting a fire is another concern. Burgoyne \& Newitt [13] investigated the effect of various explosion relief enclosures and areas on pentane-air explosions that was found to reproduce the explosion of lubricating oil mists in engine crankcases. Freeston et al. [14] studied the venting of explosions in a crankcase of a diesel engine. It was mentioned that although vent areas in the crankcase were larger than those in the experiments [13], maximum pressure in the crankcase was higher. This was likely due to the flame spreading from one cylinder compartment to another, which was noted to require some further modification. Mansfield [15] suggested a new system for adequate protection against the crankcase explosions occurring in gas engines by means of pressure relieving method. Under a program of work started at the Joint Fire Research, Rasbash \& Rogowski [16-20] initiated a systematic investigation on the relief amount for ducts conveying flammable gases. They highlighted that the best way of placing a relief vent is to distribute a given area in the form of a slot along the whole length of the duct so that wherever ignition occurs burnt products could be expelled at an early stage of the explosion. Rasbash [21, 22] discussed and evaluated the correlations of the results of different studies and pointed out the useful information available, and where further work was required. Simmonds \& Cubbage [23] tested vapor/gas explosions in industrial drying ovens and suggested requirement in designing an explosion relief for an oven manufacturer. Burgoyne \& Wilson [24] examined the effect of vent size, type of vent, and concentration of mixture on the degree of flame distortion and peak pressure in pentane vaporair explosions in cylindrical chambers. Munday $[25,26]$ conducted a performance analysis of the bursting disc valves and spring-loaded valves in the protection of pressure vessels and suggested a method to calculate the positioning and size of the valve. Munday [27] developed equations based on mass and energy balances to give realistic safe venting areas that was applicable to any initial pressure and any shape of vessel. Rogowski \& Rasbash [28] showed that the explosion of initially moving gases resulted in higher maximum pressures in ducts than those obtained with the flammable gas initially stationary. Palmer and Rogowski [29] showed that small-scale enclosures (up to $3 \mathrm{ft}^{3}$ in volume) could be safely protected against propane-air explosions with flame arresters installed in the outer casing or cover of the equipment to reduce the pressure and prevent the flame emission, provided that the thermal damage to the arresters were avoided. Further investigation by Palmer \& Rogowski $[30,31]$ suggested the use of protective cover for flame arresters to prevent accidental damage to the arresters, and ingress of moisture and dust into the equipment casing and 
measured the role of various types of covers in the explosion pressures. Charney [32] performed a series of tests to compare explosively operated vents and rupture disks. Harris \& Briscoe [33] investigated the effect of bursting disc operating pressure, fuel concentration and vent size on the maximum pressure in pentane vapor-air explosions. Griffiths et al. [34] reported that an immediate cause of the collapse of flats at Ronan Point was gas explosion that blew out the concrete panels forming part of a load-bearing flank wall. It was stated that explosions of this magnitude must be expected in domestic buildings. Rasbash [35] conducted a detailed analysis of possible explosion scenarios in a domestic structure and pointed out advice on the design of explosion relief and structure of the flat. Theoretical and experimental research conducted by Yao et al. [36] provided the explosion venting design guideline to determine the adequacy of explosion protection in gloveboxes. The mathematical model derived in the work was capable of predicting pressure-time history for a variety of venting arrangements, such as free-vent, bursting diaphragm relief venting (see also [37]) and filter venting. The ASME [38] established a code for design and installation of pressure-relief devices for protection against overpressure in pressure vessels. Gas explosions in multiple compartments, imitating conditions occurring in domestic premises, were experimentally studied by Rasbash et al. [39]. Decker [40] discussed the principles of confined gas explosions and relief of explosions and provided a venting formula for the design of explosion relief. Weldon [41] proposed the use of explosion venting in achieving damage limiting construction within plants. Runes [42] developed a procedure for estimating the required vent size in protecting a building from an internal explosion. Howard [43] analyzed the Monsanto plant accident using the Runes equation [42]. He highlighted that the importance of accounting for the increase in flame velocity likely to occur in a large-scale incident. Astbury et al. [44] performed experiments to test the effectiveness of venting in the form of cladding and windows on the structural performance of a brick building, as well as a concrete bunker, during gas explosions. Cubbage \& Marshall [45] investigated the venting effectiveness of orifice and flue on the pressure generated in industrial appliances. Dragosavic [46] performed gas explosion tests in a concrete structure comprising of two rooms, which in respect of shape and size were comparable to a kitchen and a room in a flat. The main goal of the study was to analyze the effect of venting wall of different sizes and strengths on the explosion loading and determine design requirements for loadbearing members putting into consideration the possibility of explosion. In another work, Cubbage \& Marshall [47] carried out the experiments on a cubical box and a rectangular enclosure and derived a formula that could 
assess the effectiveness of an explosion relief on the pressure developed. Howard \& Russel [48] provided a procedure for calculating venting requirements including various factors influencing the explosion venting process. Butlin \& Tonkin [49] performed an experimental study on gas explosions in a vented compartment. Particularly, they analyzed the effect of layer depth of natural gas-air mixture on the explosion pressures with three vent areas and also the effect of blast waves on the surrounding by measuring the pressure and sound level outside the chamber. Butlin [50] reviewed the available knowledge on the venting of gas explosions and made recommendations for future works. Zalosh [51] considered the use of explosion venting in a shredder and emphasized the need to determine whether the venting guidelines available by then were applicable to such environment. Sapko et al. [52] derived the equations that could predict the unrestricted venting requirements for spherical explosions of hydrocarbon vapor-air mixtures in various enclosure geometries, with unburnt or burnt gas discharge. The equations were compared with experiments on unrestricted venting experiments by Yao et al. [36]. A safe value in each comparison has been estimated. Chappell [53] described the sequence of events in gas explosions in vented confined spaces and developed, accordingly, a model that described the pressure-time history of the process. Bartknecht [54] discussed the application of pressure relief devices in a variety of equipment such as silos, dryers, granulators and filters. Harris et al. [55] suggested the use of shatter-resistant film on glass panes of the buildings to reduce the hazard of high-velocity glass fragments showering produced on window failure as a result of explosion pressures. Rogowski [56] reviewed the empirical relationships available, to define various venting requirements. Another review of empirical equations was carried out by Marshall [57], who provided guidance on the use of most appropriate formulae. Donat [58] looked at the advantages, disadvantages, and challenges in implementing combustion venting in a container appropriately. Anthony [59] evaluated the versatility of venting formulae available by then. Bradley \& Mitcheson $[60,61]$ showed two idealized analyses, one is a simple and the other is a comprehensive computer model, for predicting the required vent area and compared them with the methods proposed by the other workers. In the following work [61], the authors of [60] subsequently compared the computer solution developed in [60] with the experimental data from variety of sources available. The comparison was in terms of the safe values for a venting parameter, a dimensionless vent area parameter they preferred to use, and explained the discrepancies noticed. Crescitelli et al. [62] developed a mathematical model using the law of conservation of mass with ideal gas assumption and an auxiliary relation 
for the burning velocity. The model simulated the pressure-time pattern for explosions in vented vessels. Zalosh [63] classified vessel size being either smaller or larger than $12 \mathrm{~m}^{3}$ in volume and performed experiments to determine venting explosion venting requirements for enclosures of different shapes and with different gas-air mixtures. An order of magnitude larger peak pressure in large-scale enclosures because of suddenly accelerated combustion or flame instability at later stages of the explosion was reported [63]. Buckland [64] performed methane-air explosions tests in a room size chamber and analyzed the effect of mixture layer depth, vent diaphragm size, and strength and ignition source on the pressures. Solberg et al. [65] stated that in the case of the use of volume scaling, a scaling that states same pressure is obtained in explosions, where the ratio between area of the vent and vessel volume to the power of 2/3 is constant, is non-conservative. They reported higher pressures obtained in the experiments of gas explosions in a $35 \mathrm{~m}^{3}$ volume than expected based on volume scaling. Bartknecht [66] reviewed the knowledge on the use of various types of relief venting devices in various types of vessels and nomograms used to determine the required vent area for various geometrical and explosion conditions. Tufano et al. [67] conducted an analysis of experimental data by a mathematical model and provided a diagram to find the venting area when the maximum allowable pressure and vent breakout pressure were known. Zalosh \& Coll [68] performed an experiment in a realistic full-scale municipal solid waste shredder to provide information for venting requirement for such configuration. The authors discussed the limitations and applicability of existing venting guidelines. Mulpuru \& Wilkin [69] constructed a simplified model to predict the property transients resulting from vented deflagration of hydrogen in the spherical or cylindrical vessel. Fairweather \& Vasey [70] have developed a mathematical model to predict the pressure-time history of gas explosions in the vented cubic or cuboid vessels. Lee \& Guirao [71] discussed the physical mechanisms that give rise to the flame velocity in a vented vessel, and they suggested considerations for design and process of the system that will suppress flame acceleration. Solberg [72] provided a model that could account for flame acceleration and difficult geometries in the explosion-venting process. Howard \& Karabinis [73] performed experiments to analyze performance of different types of vent panels and different configurations of restraining the panels in propane-air explosion in a test building. They assessed the use of the Runes equation [42] for calculating necessary explosion venting areas and offered a guideline for use in the design of explosion-vented process buildings. Molkov \& Nekrasov [74] performed experiments in an almost spherical shape of vessel and presented a set of ordinary 
equations that described the dynamics of gas combustion in a constant volume with escape. The model was compared with the experiments and the results were assessed. Wingerden \& Zeeuwen [75] concluded that the maximum pressure they obtained in their rectangular vessel was due to acoustically driven combustion instability and this peak pressure could be eliminated by lining the interior of the vessel with damping material. Hirano [76] mentioned that one of the factors affecting the pressure variation during gas explosion in an enclosure was the flame behavior whose prediction was said not to be easy. Swift [77] derived a model from the combustion theory for vent area and maximum pressure calculations. Tests against available data indicated the model to be a conservative, predictive tool. Chippett [78] developed a computer model that accounted for the variations of the burning velocity with the pressure and temperature as well as enhancement of the flame surface area due to cell formation. The solution of the model enabled the pressure-time history prediction. McCann et al. [79, 80] investigated, experimentally, the gas dynamic processes occurring in small-scale cubical vessels during vented explosion and constructed, on the basis of the experimental data, a one-dimensional model of the gas venting process. The appearance of flame instabilities was demonstrated. Because the vessels were small-scale, the instabilities did not arise until later stages of the explosions, neither did they have significant effect on the internal pressure. Cooper et al. [81] conducted experiments in near-cubic enclosures to find the physical mechanisms responsible for the generation of pressure peaks during vented gas explosion. For ignition at the center and with low failure pressure relief panels, they identified four pressure peaks, whereas increasing the failure pressure of relief panels resulted in two pressure peaks dominating the pressure-time profiles. Kordylewski \& Wach [82, 83] investigated the influence of a tube connected to the vent of the vessel on the pressure rise of explosion. Epstein et al. [84] presented a simple model that could estimate the peak pressure resulting in sonic-vented hydrocarbon explosions in spherical vessels. The model used the assumption of spherical flame propagation but the distortion of the flame shape due to venting and hydrodynamic instabilities is left unquantified. In the following work, Swift \& Epstein [85] presented counterpart of the model [84] for sub-sonic flow mass discharge case, and also performed the experiments to test the effectiveness of different type of vents and vent locations. The results of the experiments as well as existing venting data were compared to the model of employed in the present work as well as with other predictive techniques. The model was able to accommodate explosion venting data. Harrison \& Eyre [86] conducted a series of experiments in a chamber where they measured the pressures both inside and 
outside of the chamber. They have found an external explosion occurring due to the ignition of the unburnt gas displaced outside the chamber by the internal flame movement. They noted that the external pressure could temporarily exceed the internal pressure. Kumar et al. [87] conducted experiments with hydrogen-air mixtures in a sphere that vented to a cylinder. They varied the hydrogen concentration, vent size and ignition location. Kumar et al. [88] performed hydrogen-air experiments in a sphere that vented to the atmosphere by varying the hydrogen concentration, vent size and ignition location. They utilized the model [69] to predict the experimental results. Epstein et al. [89] developed a computer program called 'DEFLAG' that was able to estimate peak pressure and predict the pressure-time history for sonic-vented tetrafluoroethylene decompositions. The model accounted for non-ideal gas effects and a factor to describe decomposition rate enhancement due to venting. Bouhard et al. [90] studied the dynamics of stoichiometric propane-air explosions in a cylindrical cylinder connected to a duct that vented the gas to the open air. Canu et al. [91] developed a mathematical model to simulate vented gas deflagrations. Subsequently, the model was compared with experimental propane-air and methane-air data in the literature for the variety of initial pressure, vessel volume of different shapes, and bursting pressure conditions. The average error in terms of maximum pressure was reported to be $10.4 \%$. In a concomitant work, Rota et al. [92] simplified, with suitable assumptions, the mathematical model [91] and reduced it to a system of three ordinary differential equations. The average error after comparison with the same data in the literature became equal to $28 \%$. DeGood \& Chatrathi [93] performed experiments of vented propane-air deflagrations to investigate the factors influencing the maximum pressure such as vent burst pressure, vent mass, ignition location, and discharge ducting. Catlin [94] conducted an experimental study to analyze the influence of vent size and fuel gas reactivity on the external explosion overpressure. Molkov et al. [95, 96] suggested a formula calculating a factor accounted for differences between real and simulated flame fronts. Ural [97] developed a model of explosion venting through ducts and compared it with experiments. Carcassi \& Fineschi [98] performed vented hydrogen deflagration experiments and showed that the overpressures in the compartments depended on the vent opening dimensions and the relative position of openings and igniters. Mercx et al. [99] investigated the venting of methane-air explosions in heating plants and examined the effects of vent area, vent opening pressure, ignition location and venting through a dormer window. Molkov [100] investigated the influence of venting of deflagrations through exhaust duct to a receiver, both numerically and experimentally. Alexiou et al. [101] investigated the effect of the 
vent size on the overpressure development in a large length to diameter vessel. Siwek [102] reviewed the vent calculation methods for combustible gases. Lautkaski [103] reviewed the general knowledge on the vented gas explosions. Alexiou et al. [104] conducted experiments to test the effectiveness of side venting in a large length to diameter vessel. Molkov et al. [105], with their model [74], processed the experiments from the variety of sources including various vessel volumes, gas-air mixtures, vent opening pressures, vent inertias, ignition condition and vessel shape. They were able to express all vented deflagrations under a universal correlation. Molkov [106] described the dependence of the factor that accounted for the difference between real and modeled flame on the explosion conditions. Cooper [107] discussed the effects of vent inertia in design and the consequence of high inertia vents on weak vessels. Hochst \& Leucket [108] performed a set of experiments to study the effect of mass inertia of venting panel. Molkov [109] performed simulations with the model developed in [74] to analyze the real accidents in a building and a plant. With the estimated values of minimum and maximum explosion pressures in such structures, Molkov was able to determine the appropriate range of real to laminar flame front area ratio. Ponizy \& Leyer $[110,111]$ investigated the role of duct venting connected to a vessel and varied ignition site, membrane rupture, the diameter and length of the discharge duct as parameters. Molkov et al. [112] conducted modelling and experiments of hydrogen-air deflagrations in vented vessels and compared the results. In a study of explosion initiated in a vessel and vented through a duct, Ponizy \& Veyssiere [113] studied the mitigating effect of wire-net inserts on the explosion. Chow et al. [114] reported a test study of vented explosions in a cylindrical tube. They investigated the effects of vent area, relief failure pressure, ignition position and fuel type on the flame speeds and overpressures. Forcier \& Zalosh [115] reviewed the existing data correlations for the external pressures and explored the application of blast wave models. Molkov et al. [116] processed further experiments on vented hydrocarbon-air and hydrogen-air deflagrations with the use of their model [74] and obtained new data on the factor accounting for difference in real and modelled flame areas. Razus \& Krause [117] considered different methods for the vent area calculations and their range of validity. Molkov [118] suggested some correlations for the vent sizing considering vented deflagrations at elevated pressures. Tamanini [119] discussed the correlation formulas for scaling of reduced pressure. Molkov et al. [120-123] conducted the modelling of hinged, spring-loaded, and translating inertial vent covers and reviewed the previous studies on vented gas deflagrations with inertial vent covers. Ferrara et al. [124] investigated duct-vented explosions using propane- 
air mixture and with central and rear ignition. Jiang et al. [125] analyzed the variations of the secondary (external) explosion at different vent failure pressures, ignition locations and equivalence ratios of the fuel. Hu et al. [126] studied the interaction of the flame front with the flow induced by venting. Chen et al. [127] performed a series of vented explosion tests in a smallscale cylindrical vessel using a short discharge duct and examined the effects of vent burst pressure, ignition location and equivalence ratio on external explosions. Kumar [128] performed vented experiments with hydrogen-air flames to determine their behavior in rectangular enclosure for various igniter location. Ferrara et al. [129] modelled the gas explosions vented through ducts by a two-dimensional (2D) computational fluid dynamics (CFD) model that was based on the unsteady Reynolds Averaged Navier Stokes (RANS) approach. Du et al. [130] investigated the venting process influenced by the equivalence ratio and ignition position. Janovski et al. [131] performed the methane-air experiments in an experimental mine and compared the results with their own numerical simulations. European Standard [132] recommended the use of venting panels in all civil engineering works. Molkov et al. [133] conducted an analysis of experimental deflagration in a vented enclosure with consequent analysis of large eddy simulations (LES) of the same test; a comparison of the simulated and experimental pressure transients and dynamics of the flame inside and outside the enclosure was performed. Willacy et al. [134] investigated the effects of concentration, ignition position and injection position on the stratified propane-air explosions in a vessel vented through a duct. Russo \& Benedetto [135] reviewed the available experimental and theoretical results on gas explosions in the vessels vented through a duct to study the effect of ducting on explosions. Molkov et al. [136] developed an LES model for a vented explosion of a hydrogen-air mixture and validated the model via experiments. Karnesky et al. [137] applied a three-dimensional (3D) model for prediction of maximum pressure rise in vented gaseous combustion. Kasmani et al. [138, 139] investigated the effects of vent burst pressure and ignition location on the overpressure generated by the methane and propane air explosions in a duct-vented cylindrical vessel. British Standard [140] specified the basic requirements of gas explosion venting systems for different types of enclosures. Willacy [141] investigated systematically the influence of homogeneous and stratified mixtures in vented explosions by varying the mixture reactivity, ignition position, injection position and mixture composition. Ferrara et al. [142] investigated, experimentally, the interaction between the internal and external processes for the gas explosions vented through a duct. A critical review of venting correlations adopted in standards in the USA 
and European was carried out by Kasmani [143]. Zalosh [144] provided a literature review and an analysis of the key aspects of vented gas explosions. Bauwens et al. [145] performed a series of tests on the deflagration of methane-air mixtures in a vented vessel. The experimental data was used to test a 3D model for the simulation of gaseous combustion in vented enclosures. Proust \& Leprette [146] listed a number of experiments where an external explosion resulting from the formation and ignition of a combustible cloud outside the vent overcame the internal one. Bauwens et al. [147] performed the experiments and computational simulations of stoichiometric propaneair mixtures in a vented explosion chamber, with the simulations capturing the acoustics observed in the experiments. Kasmani et al. [148, 149] investigated the effect of the vessel volume and equivalence ratio on the overpressure for vented gas explosions in a cylinder. Bauwens et al. [150] investigated the effect of ignition location and vent size on pressure transients of stoichiometric propane-air vented explosions in a room size enclosure. Lowesmith et al. [151] investigated the effect of hydrogen concentration on vented methane/hydrogen explosions in a large-scale enclosure $\left(69 \mathrm{~m}^{3}\right)$. Fakandu et al. [152] performed the experiments of vented hydrogen explosions in a 9-liter vessel and compared the results with the predictions of venting design standards. Bauwens et al. [153] performed and compared the experiments and simulations with hydrogen mixtures, and then compared to those for propane and methane. Chao et al. [154] investigated the effect of fuel, enclosure scale, ignition location and vent size on pressure development for vented gas deflagrations. They reported that the maximum pressures were caused by the interplay of the external explosion, acoustics, and maximum flame area. Fakandu et al. [155] reported that the design procedures for the protection of explosions using venting was inadequate for hydrogen-air explosions. Bauwens et al. [156] focused on the lean hydrogen-air deflagrations with varying the ignition location, hydrogen concentration and vent size. Fakandu et al. [157] investigated the influence of vent distribution for the same total area of vent in the vented methane- and ethyleneair explosions. Sustek \& Janovsky [158] selected a set of equations and a variety of experimental data and calculated the maximum overpressures. Daubech et al. [159] performed experiments of hydrogen-air explosions in a rectangular chamber considering various reactivity, vent size and ignition position. Fakandu et al. $[160,161]$ investigated the influence of non-central and multiple distributed vents on the explosion pressure as well as on the vent areas, vent static burst pressures, fuels and concentration, considering central and rear (far-end opposite the vent) ignition locations. Schiavetti et al. [162] conducted experiments with hydrogen ignited inside and outside the vented 
volume, examining pressure evolution for each condition. Rocourt et al. [163] performed experiments on vented hydrogen-air explosions in a small, enclosed volume. They investigated the effects of vent area and ignition location on the pressure peaks. Guo et al. [164] particularly focused on the effect of ignition position on the vented hydrogen-air explosions. Hernandez et al. [165] analyzed the experimental data of Ref. [108] to establish the burning rate based on a simplified model for vented combustion. Tomlin et al. [166] performed experiments to determine the effect of the vent size on the vented natural gas explosions. Fakandu et al. [167] investigated the effect of vent burst pressure on the overpressure generated in a cylindrical vented vessel. Guo et al. [168, 169] investigated, experimentally, the effect of the vent burst pressure on rich hydrogen- and methane-air explosions. Qi et al. [170] studied the role of the vent size and fuel concentration in the pressure development and flame behavior inside and outside a vessel during vented gasoline-air explosion. A computational platform for hydrogen-air and methane-air vented gas explosions (EVA) was developed by Ugarte et al. [171] and Sezer et al. [172].

Currently, there are numerous studies on vented gas explosion available in the literature. However, the intended application of each of these individual studies (some of them were mentioned) have usually their own particular specifications such as geometry type, fuel contained, layout of the enclosure, location of possible ignition sources, maximum allowable pressure, venting details, biosafety hazard level of containment and surrounding concerns. Furthermore, the enclosures that represent the application can be rather large in the volumes of tunnels, subways or coalmines, for which conducting experiments or performing simulations that account the smallest scale resolution of flame is expensive from the point of view of the experimental and computational resources. One of the standards defining venting requirements is by a global organization, National Fire Protection Association (NFPA), whose 2018 updated standard (NFPA 68) [173] recommends the following equation in determining the minimum required vent area, $A_{v 0}$, (in $\left.\mathrm{m}^{2}\right)$ in vented gas deflagrations:

$$
A_{v 0}=A_{s} \frac{\left[1-\left(\frac{P_{r e d}+1}{P_{\max }+1}\right)^{1 / \gamma_{b}}\right]}{\left[\left(\frac{P_{r e d}+1}{P_{\max }+1}\right)^{1 / \gamma_{b}}-\delta\right]} \frac{S_{u} \rho_{u}}{G_{u}} \frac{\lambda}{C_{d}},
$$

where 


$$
\delta=\frac{\left(\frac{P_{\text {stat }}+1}{P_{0}+1}\right)^{1 / \gamma_{b}}-1}{\left(\frac{P_{\max }+1}{P_{0}+1}\right)^{1 / \gamma_{b}}-1},
$$

and $A_{s}$ is the enclosure internal surface area $\left(\mathrm{m}^{2}\right), P_{\text {red }}$ the maximum pressure developed in a vented enclosure during vented deflagration (bar-g), $S_{u}$ fundamental burning velocity of gas-air mixture $(\mathrm{m} / \mathrm{s}), \rho_{u}$ mass density of unburned gas-air mixture $\left(\mathrm{kg} / \mathrm{m}^{3}\right), \lambda$ ratio of gas-air mixture burning velocity accounting for turbulence and flame instabilities in vented deflagration to the fundamental (laminar) burning velocity, $G_{u}$ unburned gas-air mixture sonic flow mass flux $\left(\mathrm{kg} / \mathrm{m}^{2}\right.$ s), $C_{d}$ vent flow discharge coefficient, $P_{\max }$ the maximum pressure developed in a contained deflagration by ignition of the same gas-air mixture (bar-g), $P_{0}$ the enclosure pressure prior to ignition (bar-g), $\gamma_{b}$ ratio of specific heats for burned gas-air mixture and $P_{\text {stat }}$ nominal vent burst pressure (bar-g). It is noted that Eq. (2.1) is not based on any theory but is an empirical correlation taken from the experiments. Moreover, the calculation from Eq. (2.1) depends on the determination of the factor $\lambda$ whose calculation is based also on an empirical correlation.

Rather than relying on the empirical correlation in calculation of required vent areas, there is still a need for an engineering model that is based on the theory. Consequently, the objective of the dissertation is to update an engineering model that could predict the pressure-time history of the methane-air deflagration in a vessel vented into atmosphere so that the minimum vent area could be determined accordingly by predictions. The model called the Explosion Venting Analyzer (EVA) requires only the inputs of the vessel size, vent area and mixture composition. The first step towards this was undertaken in this dissertation by performing experiments at Worcester Polytechnic Institute (WPI) and simulating such experiments with the EVA. Comparison of the results were made. Moreover, the mechanisms of the flame dynamics that could occur in a oneend closed enclosure was studied by both numerical simulations and analytical theory for unobstructed and obstructed enclosure, respectively. Ultimately, the goal of this research in Dr. Akkerman's group at West Virginia University (WVU) is extending the validity of the EVA to predict the burning accident in larger enclosures with and without obstacles such as in coalmines. The EVA was proved to predict the vented methane-air deflagration in relatively smaller enclosures reasonably well. 


\subsection{Flame Acceleration - the Effect of Heat Loss}

Careful inferences are required to reduce the risks of accidental events in facilities operating with flammable gases or combustible dust [174]. In order to prevent losses in such accidents, researchers worldwide analyze the behavior of a premixed flame in various geometries. Among various configurations of a combustor, tubes or channels are most employed in the studies of flame acceleration and deflagration-to-detonation transition (DDT) event [175]. This is (i) because a pipe/channel is the simplest to study, from a theoretical viewpoint, and (ii) because in this geometry the acceleration rate and the burning time may be highest and/or high enough to trigger detonation, especially in the case of obstructed conduits.

There are several mechanisms of flame acceleration in pipes/channels such as (i) that due to a finger shape of the flame front attained at the initial stages of burning [176]; (ii) that due to wall friction [177]; (iii) that due to obstacles built at the pipes walls [178]. For the first time, finger flame acceleration was studied experimentally by Ellis [179], for a flame in a closed cylindrical tube. However, what is better recognized are the experiments of Clanet \& Searby [176], with four different stages of flame propagation observed, namely: (i) hemispherical flame expansion, (ii) exponential acceleration of a finger-shaped flame front that lasts until the flame 'skirt' contacts the side walls, (iii) flame deceleration due to the decrease in the flame surface area, and (iv) presence of acoustic effects after the so-called 'tulip flame' formation. Initial acceleration, associated with the transition between stages (i) and (ii), occurs when an initially hemispherical flame front starts approaching the sidewalls such that the axial flow velocity exceeds the radial one considerably. The dynamics and morphology of a finger flame front along with other parameters such as the acceleration time interval, the evolution of the position and velocity of the flame tip as well as its acceleration rate have been identified, analytically and computationally, by Bychkov et al. [180]. Subsequently, the effect of gas compressibility on finger flame acceleration was investigated by Valiev et al. [181], with the conclusion that gas compression moderates the acceleration rate noticeably. The work [180] also found that the finger flame acceleration is scaleinvariant, i.e. Reynolds-independent, which makes it equally strong for micro-channels and mining passages. Demir et al. $[182,183]$ adopted the finger flame acceleration mechanism to coalmines, combining it with the Darrieus-Landau (DL) combustion instability, and concluded that up to two orders of magnitude increase in the flame propagation velocity, due to the instability plus the finger 
flame scenario may trigger detonation, which is a tremendous disaster in accidental burning events in the coalmining industry. It is noted that at large scales such as mining passages, turbulence and the other instabilities such as the Rayleigh-Taylor (RT) instability may play an important role in propagation of a finger-shaped flame front [184], thereby making it possibly Re-dependent. However, their effects on flame acceleration are still unclear.

It is noted that most of theoretical and numerical works on flame acceleration in pipes/channels [178, 180-183, 185] involve a number of simplifying assumptions such as adiabatic and free-slip or nonslip wall boundary conditions. However, the walls are neither adiabatic nor free-slip in practice, and wall conditions may significantly impact flame propagation. Han et al. [186] have considered adiabatic and non-slip open channel with a planar flame ignition at the closed end of the channel. They evaluated different width sizes, namely micro- and macro-channels, and compared the two different modes of flame propagation. Ivanov et al. [187] performed numerical simulations of hydrogen-oxygen flame acceleration in two-dimensional (2D) channels of various widths, using a detailed chemical model. Dzieminska \& Hayashi [188] studied the shock wave boundary layer interaction for the auto-ignition in the boundary layer for unobstructed channels with adiabatic and non-slip boundary conditions. In their simulations, the authors of Ref. [188] employed a detailed chemical kinetics model with eight (8) species and eighteen (18) reactions. It is concluded that cooling down of the walls may prevent overheating of the boundary layer and possible DDT formation. Machida et al. [189] performed 3D numerical simulations of flame propagation in a rectangular channel filled with hydrogen-oxygen mixture using detailed chemical reaction model. The authors of Ref. [189] employed isothermal and non-slip boundary conditions and compared the 3D results with 2D simulations of Fukuda et al. [190] highlighting that the flames propagated qualitatively similar in 2D and 3D geometries until the transition to detonation but, the time and the location of the local explosions were different. Another comparison of 3D and 2D simulations of flame propagation in a channel was conducted by Ivanov et al. [191], where the authors stated that the 2D cross-sections of the 3D simulations showed similar hydrodynamic flow pictures to the ones obtained in the $2 \mathrm{D}$ simulations. The effect of the heat losses through the walls and the difference between free-slip and nonslip wall conditions have been studied for the two acceleration mechanism, namely, that due to (i) obstacles [178] and (ii) wall friction in the unobstructed channels [192,193]. In the obstructed channels, the numerical study [178] compared the isothermal and adiabatic walls and showed that the thermal boundary conditions did not 
provide any significant effect on the flame characteristics, since the flame propagated away from the walls in the presence of obstacles, which differed significantly from the flame behavior in unobstructed, smooth-walled channels. Regarding the unobstructed conduits, Ott et al. [194] considered flame propagation in 2D channels filled with premixed acetylene and air and with a planar flame ignition in the closed end of a channel with one end closed and one open. They evaluated the adiabatic and isothermal wall boundary conditions and arrived to the conclusion that growth of the boundary layer promoted the flame propagation speed for both thermal boundary conditions. However, the acceleration in isothermal walls was slower and, in some cases, heat losses even led to deceleration. Gamezo \& Oran [193] investigated the influence of wall temperature on propulsion characteristics and pointed out the importance of the selection of an insulation material on the efficiency of a propulsion device. The model [193] was capable of introducing three types of boundary conditions, namely: adiabatic and isothermal walls as well as the walls set with a heat loss coefficient, which can differ to represent different insulation materials. The work [193] clearly demonstrates that the adiabatic and isothermal conditions are two limits, while practical reality is between these limits. It was found that with the increase in such a heat loss coefficient, the heat loss from the burnt matter to the walls mitigates flame acceleration. For isothermal cases, no significant acceleration was reported. Overall, isothermal walls provide a considerable impact on flame acceleration induced by wall friction: the exponential acceleration trend inherent to adiabatic conditions [185] is replaced by at most linear acceleration for isothermal wall conditions. Moreover, extinction may occur when a channel is very narrow. Hackert et al. [195] also investigated the effects of thermal boundary conditions on the flame shape and velocity, with a particular focus on a flame quenching distance in ducts. It was noted that the closer the duct to the adiabatic condition, the faster the flame is; however, the effect of internal radiation may further increase the burning rate even in the case of adiabatic walls. Specifically, internal radiation diminishes the quenching distance and promoted flame acceleration, while external radiation promotes the heat transfer and increases the quenching distance. Among other studies to be mentioned, Brailovsky \& Sivashinsky [196] developed a theoretical model to describe flame acceleration by introducing a friction parameter for burning in tubes, and they suggested that hydraulic resistance exerts a destabilizing impact on flame propagation that can cause a transition from uniform propagation to gradual acceleration. While the model [196] neglected heat losses, Kagan et al. [192] extended the study [196] to account for this effect and concluded that 
heat losses tend to reverse the effects of hydraulic resistance and that the impact of heat losses is as strong as that of hydraulic resistance. Norton \& Vlachos [197] reported that the channel/tube walls with high thermal conductivity lead to a larger hot area for external heat transfer, making the global-like extinction more susceptible. Daou \& Matalon [198] stated that the excessive heat losses can cause a total flame extinction in narrow channels, but only partial flame extinction in wider ones. In the latter case, a flame persists in the center of a channel. Gauthier \& Bergthorson [199] considered various convective heat transfer coefficients at the inner and outer wall surfaces of the tubes of different diameters and concluded that an increase in the external heat loss reduces the flame propagation velocity.

It is seen that the impact of the wall conditions on flame propagation can be significant, especially when a flame propagates near a wall. Will it be the case for finger flame acceleration at the initial stages of burning in channels/pipes? The previous works on finger flame acceleration [180-183] could not answer this question as they employed adiabatic, free-slip walls. Consequently, this point is addressed in the second subsection of the results section by means of extensive computational simulations of the reacting flow equations with fully-compressible hydrodynamics and chemical kinetics imitated by one-step Arrhenius reaction.

\subsection{Fire Scenario in an Obstructed Mining Passage}

Silvestrini et al. [200] developed a simplified formula evaluating the flame run-up distance (a distance that a flame propagates before a detonation initiation) for smooth tubes and then extended it to account for obstacles, which have been shown to promote flame acceleration, thereby reducing the run-up distances. Ciccarelli \& Dorofeev [175] reviewed the knowledge on flame acceleration in unobstructed and obstructed channels, emphasizing formation of a cellular flame structure due to the DL instability, as observed in the small-scale experiments for flames in obstructed channels. Houim \& Oran [201] considered the channels with smooth walls, filled with a coal dust or ash particles, layered at the bottom of a channel. It was shown, computationally, that flame interaction with the coal dust formed hot spots ahead of the flame front; autoignition of these hot spots produced the jumps in the flame position and sharp spikes in the flame velocity. Seshadri et al. [202] considered the structure of a premixed flame propagating in the environments where combustible particles are uniformly distributed and identified the effective equivalence ratio and laminar burning velocity of the mixture in terms of particle size and concentration. Xie et al. [203] 
showed, experimentally, that a small size of the combustible coal particles promotes the overall equivalence ratio of the fuel mixture as well as the laminar burning velocity in this mixture. Zheng et al. [204] investigated the effect of the distribution of the methane-air fuel mixture inside a 2D obstructed channel on the DDT event, with a finding that inhomogeneous distributions either did not produce a detonation or resulted in a detonation that was decoupled into a flame and a shock earlier as compared to the homogeneous distribution. The wall restrictions in the confined enclosures as well as the shape and layout of the obstacles can significantly influence flame acceleration in obstructed channels. In unconfined spaces, Ogawa et al. [205] showed that an array of square obstacles led to continuous detonation propagation. Bychkov et al. [206] provided an explanation of a physical mechanism of flame acceleration in a $2 \mathrm{D}$ obstructed channel, equipped with a comb-shaped array of tightly-placed obstacles. It was demonstrated that delayed burning in the "pockets" between the obstacles leads to a "piston-like" motion pushing forward the flow ahead of the flame in the unobstructed part of the channel. Valiev et al. [207] extended the 2D study [206] into the cylindrical-axisymmetric geometry, with much stronger flame acceleration found in the cylindrical tubes as compared to the 2D channels. The work [208] recognized that various obstructions, which are inevitably present in a coalmine (such as mining equipment, belt conveyor systems, and piles of rubbles), may block a noticeable part of the passage, thereby providing a significant impact on a fire scenario. There is therefore a critical need to account for the obstacles in a predictive coalmining fire scenario, and this question is particularly addressed in the third subsection of the results section. 


\section{Methodology}

This section describes the computational platforms employed in this dissertation. For the deflagrative burning of a combustible mixture in a volume that vents to a larger environment, the Explosion Vent Analyzer (EVA) was used to predict the transient properties of the process. To study the effect of boundary conditions, computational simulations of the compressible NavierStokes equations for unsteady reactive flows were performed by means of the Gas Explosion Model (GEM).

\subsection{Mathematical Description of EVA}

EVA is a zero-dimensional model that is used to predict the property transients resulting from deflagration of a combustible mixture in an enclosure with a venting of the gas mixture to the environment. Derivation of the model is based on the following assumptions:

- Premixed homogeneous mixture is point ignited in the volume.

- An infinitely thin, smooth, spherically symmetrical flame propagates outwards from the point of ignition.

- Properties of the burnt and unburnt mixtures and pressure are distributed uniformly in the volume.

- All compressions and expansions of the unburnt mixture are isentropic.

The backbone of the formulation in the EVA is constituted by Mulpuru \& Wilkin [69]. The mass balance consists of three terms,

$$
\frac{d}{d t}\left(\frac{m_{u}}{m_{i}}\right)+\frac{d}{d t}(n)+\frac{d}{d t}\left(\frac{m_{v}}{m_{i}}\right)=0,
$$

where the subscripts $u, b, v$ and $i$ represent unburned, burned, vented and initial conditions while $m$ and $t$ stand for mass and time, and $n\left(m_{b} / m_{i}\right)$ is the fraction of the initial burnt mass. By using the isentropic relation $P / \rho^{\gamma_{u}}=$ const., rate of change of the first term in parenthesis is related as:

$$
\frac{m_{u}}{m_{i}}=\bar{P}^{1 / \gamma_{u}}(1-\bar{V}),
$$


where $\bar{P}=P / P_{i}$ is the instantaneous to initial pressure ratio; $\gamma_{u}=\left(C_{P} / C_{v}\right)_{u}$ is the specific heat ratio of unburned gas; $\bar{V}=V_{b} / V_{i}$ is the instantaneous to initial volume ratio of the burned gas. The vented mass rate is expressed by the orifice discharge relations:

$$
\frac{d}{d t}\left(\frac{m_{v}}{m_{i}}\right)=C_{d} \frac{A_{v} \rho}{m_{i}}\left[\frac{P}{\rho}\left(\frac{\gamma+1}{2}\right)^{((\gamma+1) /(\gamma-1))}\right]^{1 / 2},
$$

for the choked condition, $P_{a} / P \leq 1 / \bar{P}_{\text {critical }}$, and

$$
\frac{d}{d t}\left(\frac{m_{v}}{m_{i}}\right)=\frac{C_{d} A_{v}}{m_{i}}\left[\frac{2 \gamma P \rho}{\gamma-1}\left(\frac{P_{a}}{P}\right)^{2 / \gamma}\left[1-\left(\frac{P_{a}}{P}\right)^{(\gamma-1) / \gamma}\right]\right]^{1 / 2},
$$

for the subsonic condition, $P_{a} / P>1 / \bar{P}_{\text {critical }}$; where the $C_{d}$ is the discharge coefficient, $A_{v}$ the vent area, $\bar{P}_{\text {critical }}=((\gamma+1) / 2)^{\gamma /(\gamma-1)}$ the critical pressure, and $P_{a}$ the exit pressure being constant until the occurrence of external explosion. The rate of burnt mass formation as a fraction of initial mass is formulated according to the definition of burning velocity

$$
\frac{d}{d t}(n)=\frac{1}{m_{i}} \rho_{u} S A,
$$

where $S$ is the burning velocity and $A$ is the flame surface area to be modeled. By using the isentropic relation for unburned gas density, Eq. (3.5) can be modified as

$$
\frac{d}{d t}(n)=\frac{A}{V_{i}} S_{T} \bar{P}^{1 / \gamma_{u}}-K \frac{d}{d t}\left(\frac{m_{v}}{m_{i}}\right)_{b},
$$

where the factor $K$ is a switch indicating if unburned $(K=0)$ or burned $(K=1)$ gas is venting. The conservation of energy is expressed as:

$$
\frac{d}{d t}\left(\frac{m_{u}}{m_{i}} E_{u}\right)+\frac{d}{d t}\left(n E_{b}\right)+E_{u} \frac{d}{d t}\left(\frac{m_{v}}{m_{i}}\right)=0,
$$

where $E=e_{0}+\left[e(T)-e\left(T_{0}\right)\right]$ accounts for the energy of formation $e_{0}$ and the change of internal energy from a reference temperature. By substituting this relation into Eq. (3.7) and using the continuity equation (3.1) and ideal gas relations, the following equation is obtained:

$$
\frac{d}{d t}(\bar{P}(1-\bar{V}))+\frac{\gamma_{u}-1}{\gamma_{b}-1} \frac{d}{d t}(\bar{P} \bar{V})=b \frac{d}{d t}(n)-\bar{P}^{\left(1-1 / \gamma_{u}\right)} \frac{d}{d t}\left(\frac{m_{v}}{m_{i}}\right),
$$

where $b=\left(e_{u 0}-e_{b 0}+T_{0}\left[\left(C_{v}\right)_{b}-\left(C_{v}\right)_{u}\right]\right) /\left(\left(C_{v}\right)_{u} T_{i}\right)$ is constant. If burned gas is vented, then Eq. (3.8) becomes: 


$$
\frac{d}{d t}(\bar{P}(1-\bar{V}))+\frac{\gamma_{u}-1}{\gamma_{b}-1} \frac{d}{d t}(\bar{P} \bar{V})=\frac{d}{d t}(n)+\left[b-\frac{\gamma_{u}-1}{\gamma_{b}-1} \frac{\bar{P} \bar{V} m_{i}}{m_{b}}\right]\left(\frac{m_{v}}{m_{i}}\right)_{b},
$$

Equations (3.1), (3.6), and (3.8) constitute the set of three non-linear ordinary differential equations with three unknowns, $\bar{P}, n$ and $\bar{V}$ for a combustion process inside an enclosure. For convenience these equations are written together below

$$
\begin{gathered}
\frac{d \bar{P}}{d t}=\frac{B_{1} A_{22}-B_{2} A_{12}}{A_{11} A_{22}-A_{12} A_{21}}, \\
\frac{d \bar{V}}{d t}=\frac{B_{2} A_{11}-B_{1} A_{21}}{A_{11} A_{22}-A_{12} A_{21}}, \\
\frac{d n}{d t}=\left(A / V_{i}\right) \bar{P}^{\left(1 / \gamma_{u}\right)} S ; \text { for unburnt gas venting } \\
\frac{d n}{d t}=\left(A / V_{i}\right) \bar{P}^{\left(1 / \gamma_{u}\right)} S-\frac{d}{d t}\left(\frac{m_{v}}{m_{i}}\right) ; \text { for burnt gas venting }
\end{gathered}
$$

where

$$
\begin{aligned}
& A_{11}=(1-\bar{V}) \bar{P}^{\left(1 / \gamma_{u}-1\right)} / \gamma_{u}, \quad A_{12}=-\bar{P}^{\left(1 / \gamma_{u}\right)}, \quad A_{21}=1+\bar{V}\left(\gamma_{e}-1\right), \quad A_{22}=\bar{P}\left(\gamma_{e}-1\right), \\
& B_{1}=-\frac{d n}{d t}-\frac{d}{d t}\left(\frac{m_{v}}{m_{i}}\right), \quad B_{2}=b \frac{d n}{d t}-\bar{P}^{\left(1-1 / \gamma_{u}\right)} \frac{d}{d t}\left(\frac{m_{v}}{m_{i}}\right) ; \text { for unburnt gas venting, } \\
& B_{2}=b \frac{d n}{d t}+\left(b-\gamma_{e} \frac{\bar{P} \bar{V}}{n}\right) \frac{d}{d t}\left(\frac{m_{v}}{m_{i}}\right) ; \text { for burnt gas venting, } \quad \gamma_{e}=\left(\gamma_{u}-1\right) /\left(\gamma_{b}-1\right), \quad b=\gamma_{e} \bar{P}_{f}-1 .
\end{aligned}
$$

The non-linear differential equations (3.10) - (3.13) are of the form

$$
\frac{d \vec{y}}{d t}=f(\vec{y}, t)
$$

where $\vec{y}$ is a vector of dependent variables and $t$ is the independent variable. Along with auxiliary relations to model flame velocity, flame surface area and external explosion, the equation set (3.14) was solved numerically using $4^{\text {th }}$-order accurate, explicit Runge-Kutta method.

\subsection{Numerical Description of the GEM}

The governing equations are those for the balance of mass (continuity), momentum, energy, and species. For a 2D Cartesian geometry they read: 


$$
\begin{gathered}
\frac{\partial}{\partial t} \rho+\frac{\partial}{\partial x_{i}}\left(\rho u_{i}\right)=0 \\
\frac{\partial}{\partial t}\left(\rho u_{i}\right)+\frac{\partial}{\partial x_{j}}\left(\rho u_{i} u_{j}+\delta_{i, j} P-\zeta_{i, j}\right)=0 \\
\frac{\partial}{\partial t}\left(\rho \varepsilon+\frac{1}{2} \rho u_{i} u_{j}\right)+\frac{\partial}{\partial x_{i}}\left(\rho u_{i} h+\frac{1}{2} \rho u_{i} u_{j} u_{j}+q_{i}-u_{j} \zeta_{i, j}\right)=0 \\
\frac{\partial}{\partial t}(\rho Y)+\frac{\partial}{\partial x_{i}}\left(\rho u_{i} Y-\frac{\mu}{S c} \frac{\partial Y}{\partial x_{i}}\right)=-\frac{\rho Y}{\tau_{R}} \exp \left(-E_{a} / R_{u} T\right)
\end{gathered}
$$

where $Y$ represents the mass fraction of the fuel mixture; $\varepsilon=Q Y+c_{v} T$ is the specific internal energy; $h=Q Y+c_{p} T$ the specific enthalpy; $Q=c_{p} T_{f}(\Theta-1)$ the specific energy release from the reaction, where $\Theta=\rho_{f} / \rho_{b}=T_{b} / T_{f}$ designates the thermal expansion in the burning process. As for other parameters in Eqs. (3.15) - (3.18), $c_{v}=5 R_{u} / 2 M$ and $c_{p}=7 R_{u} / 2 M$ are the specific heats at constant volume and pressure, respectively, where $R_{u}=8.31 \mathrm{~J} /(\mathrm{mol} \cdot \mathrm{K})$ is the universal gas constant, and the molar masses of the fuel-air mixture and the burnt matter are assumed to be equal, $M=2.9 \times 10^{-2} \mathrm{~kg} / \mathrm{mol}$. The ideal gas model is used for both the burnt and unburned gases such that the equation of state reads

$$
P=\rho R_{u} T / M,
$$

with the initial pressure $P_{f}=10^{5} \mathrm{~Pa}$, temperature $T_{f}=300 \mathrm{~K}$, and density $\rho_{f}=1.16 \mathrm{~kg} / \mathrm{m}^{3}$. The stress tensor $\zeta_{i, j}$, and the energy diffusion vector $q_{i}$ are given by

$$
\zeta_{i, j}=\mu\left(\frac{\partial u_{i}}{\partial x}+\frac{\partial u_{j}}{\partial x_{i}}-\frac{2}{3} \frac{\partial u_{k}}{\partial x_{k}} \delta_{i, j}\right), \quad q_{i}=-\mu\left(\frac{c_{p}}{\operatorname{Pr}} \frac{\partial T}{\partial x_{i}}+\frac{Q}{\operatorname{Sc}} \frac{\partial Y}{\partial x_{i}}\right),
$$

where $\mu=2.38 \times 10^{-5} \mathrm{~N} \mathrm{~s} / \mathrm{m}^{2}$ is the dynamic viscosity, and Pr and Sc are the Prandtl and Schmidt numbers, respectively. To avoid the diffusional-thermal instability, in the present work $\mathrm{Pr}=\mathrm{Sc}=0.7$ such that their ratio (the Lewis number) is unity, $L e=\operatorname{Pr} / \mathrm{Sc}=1$. In order to avoid the influence of gas compressibility, a relatively low Mach number was employed in such a way that $M a \equiv U_{f} / c_{s}=10^{-3}$, where $c_{s}=347 \mathrm{~m} / \mathrm{s}$ is the speed of sound. From this relation, the unstretched laminar flame velocity was calculated to be $U_{f}=34.7 \mathrm{~cm} / \mathrm{s}$. Once $U_{f}$ is known, the flame thickness $L_{f}$ was conventionally defined as

$$
L_{f}=\mu / \operatorname{Pr} \rho_{f} U_{f} \approx 5.9 \times 10^{-5} \mathrm{~m} .
$$

According to Eq. (3.18), the chemical reaction was imitated by a one-step irreversible reaction obeying the Arrhenius law, with the activation energy $E_{a}$ and the constant of time dimension $\tau_{R}$. 
In the present work, the activation energy was employed as $E_{a}=7 R_{u} T_{b}$. The factor $\tau_{R}$ was adjusted to obtain a particular value of the planar flame velocity $U_{f}$ by solving the associated eigenvalue problem [185]. For example, for $U_{f}=34.7 \mathrm{~cm} / \mathrm{s}$ the constant of time dimension was set to be $\tau_{R}=4.06 \times 10^{-8} \mathrm{~S}$. As for the total burning rate, similar to Refs. [178], in this work it was calculated as

$$
U_{w}=\frac{1}{\rho_{f} H} \int \frac{\rho Y}{\tau_{R}} \exp \left(-E_{A} / R_{u} T\right) d x d z .
$$

Another important characteristic of flame propagation is the flame tip velocity in the laboratory reference frame, $U_{t i p} \equiv d Z_{t i p} / d t$.

A flame propagates in a long $2 \mathrm{D}$ channel of width $2 H$. In this respect, the major dimensionless quantities of this study are the Reynolds number associated with flame propagation $\operatorname{Re} \equiv H S_{L} / v=$ $H / L_{f} \operatorname{Pr}=H / L_{f}$ (being actually the scaled channel half-width), the scaled time $\tau \equiv U_{f} t / H$, the instantaneous scaled flame tip position $Z_{t i p} / H$, its scaled velocity $U_{t i p} / U_{f}$, and the instantaneous scaled burning rate $U_{w} / U_{f}$.

\section{Boundary Conditions}

Different wall boundary conditions were employed in the numerical simulations to study their influence on flame acceleration. Specifically, semi-open channels were assumed such that one end is open while the other is closed, and the embryonic flame is ignited at the channel centerline, at the closed end, and it propagates towards the open one. The employed boundary conditions read:

- The free-slip walls are implemented as $\boldsymbol{n} \cdot \boldsymbol{u}=0$, where $\boldsymbol{n}$ is the normal vector to the walls.

- The non-slip walls are implemented as $\boldsymbol{u}=0$.

- The adiabatic walls are implemented as $\boldsymbol{n} \cdot \nabla T=0$.

- The isothermal wall temperature $T_{w}$ is set in the range $298 \mathrm{~K} \leq T_{w} \leq 600 \mathrm{~K}$.

\section{Numerical Scheme}

The numerical scheme employed in the solver was based on the finite-volume method, being the $2^{\text {nd }}$-order accurate in time, and the $4^{\text {th }}$-order in space for the convective terms, and the $2^{\text {nd }}$-order in space for the diffusive terms. The solver was first developed at Volvo Aero and was subsequently employed for academic use; see, for instance, Ref. [178] and references therein. The channel is assumed to be long enough such that the length does not influence flame propagation. The 
numerical mesh consists of structured rectangular grids, with the grid walls parallel to each Cartesian axis. Additionally, the grid is uniform in the z-direction. In this study, an adaptive grid with a uniform grid size of $0.2 L_{f}$ near the flame front was used. Outside the region of the fine grid, the mesh size increased gradually with $2 \%$ change in size between the neighboring cells. In order to keep the flame and pressure waves in the zone of the fine grid, a periodical mesh reconstruction has been implemented during the simulation run. Sufficiency of such a grid had been successfully tested [181]. 


\section{Results}

\subsection{Experimental Study of Gaseous Combustion}

A series of experiments were conducted at WPI to analyze flame propagation and associated pressure evolution inside a combustor. The experimental setup consisted of a cylinder, high-speed camera, pressure transducers, gas analyzer, igniter, computer, and mass flow controllers; see Figs. 4 and 5.

The results are presented in three subsections: first, a single cylinder made of a transparent polycarbonate material and that is $30 \mathrm{~cm}$ in length was used; secondly, a single cylinder made of the same material but $60 \mathrm{~cm}$ in length was used; and lastly, for future work, dual-chamber compartment with two vents $60 \mathrm{~cm}$ in length was recommended. The diameter of the cylinders in all the setups was $19 \mathrm{~cm}$. Each experimental test was carried out no less than three times and the results showed good repeatability.

\subsubsection{Experimental Procedure}

The chamber was initially filled with air. Then, the methane and the air were supplied using the mass flow controllers (Sierra SmartTrak100). In all the experiments, the methane and air gases were injected into the cylinder separately to imitate the conditions of methane accumulation inside a coalmining passage, such that the injected gases were not mixed before entering the cylinder. First, an amount of methane that is slightly higher than needed amount for the test condition was injected into the chamber from the rear center of the cylinder, with a preset mass flow rate. Subsequently, an amount of air that was needed to bring the mixture composition to the test condition and also to flush the methane gases inside the inlet hose was supplied into the chamber. Required amount of methane and air gases were provided by predetermined flow rates and predefined injection timing of both gases for both setups with single and extended cylinders. A gas analyzer (Servotough Oxy 1910B1) was used to monitor methane-air composition inside the vessel. The equivalence ratio was kept at test conditions and the steady readings from the gas analyzer were obtained for the test conditions prior to ignition, which was triggered by a spark igniter of voltage $1.5 \mathrm{~V}$, delivering $35 \mathrm{~mJ}$, located at the rear or center of the vessel. 
The experiments were video-recorded in order to scrutinize flame propagation inside the vessel. To allow an optical access, the vessel was made of transparent polycarbonate (wall thickness of 3 $\mathrm{mm}$ ), and it was attached to a steel bench with C-clamps to avoid any movement during the tests. The circular openings (vents) were arranged at one end of the cylinder, with the vent areas of $A_{v}=$ $67.9,86.6$, and $132.7 \mathrm{~cm}^{2}$. For the initial sealing, the vents were covered with a thin aluminum foil of thickness $0.03 \mathrm{~mm}$. Prior to ignition, the center of the foil was cut in the horizontal and vertical directions to provide a free-venting condition.

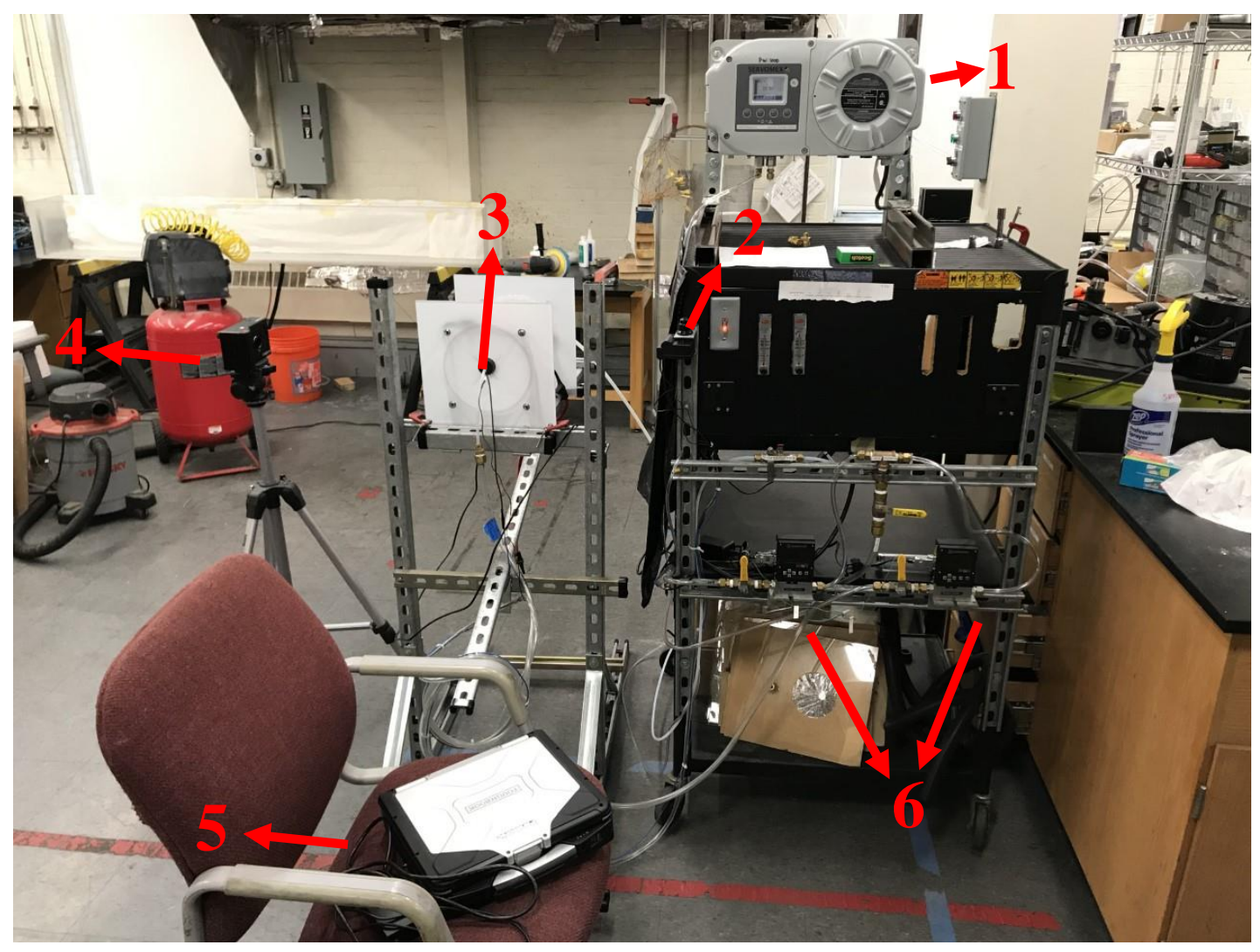

Figure 4: The experimental setup: rear view: 1) gas analyzer, 2) ignition button, 3) igniter and gas inlet, 4) high-speed camera, 5) computer, 6) mass flow controllers for air and methane. 


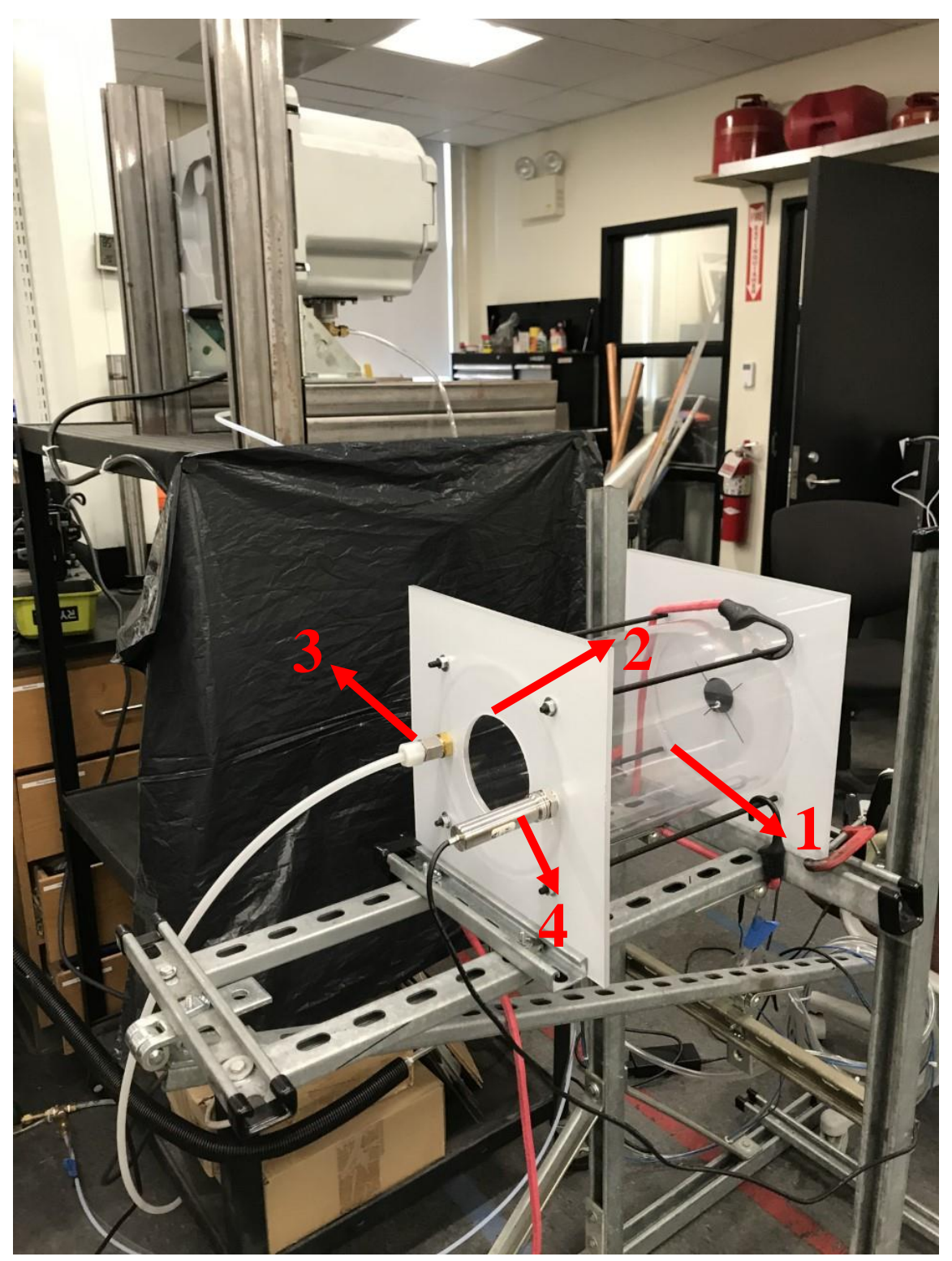

Figure 5: The experimental setup: front view: 1) transparent cylinder, 2) vent, 3) exhaust and sample line, 4) pressure transducer.

\subsubsection{Measurement Devices}

Servotough Oxy 1910B1 gas analyzer was used to measure the oxygen concentration inside the combustion chamber. This gas analyzer uses the paramagnetic oxygen sensor working principle, and it consists of a dumb bell-shaped body, a magnet, a light source, a mirror, a photocell, amplifier and a calibrated indicating unit, as shown in Fig. 6 [209]. The dumbbell-shaped body is made of glass and filled with a low magnetic susceptible gas such as nitrogen and is free to rotate in the space between the poles of the magnet. The operation principle of the gas analyzer is the high 
magnetic susceptibility of oxygen molecule, a physical property that distinguishes it from most other gases. The dumbbell-shaped body is initially suspended in a strong magnetic field due to the low magnetic susceptibility because of its nitrogen content. When a gas containing oxygen enters the measuring cell, the oxygen is attracted to strong magnetic field, causing the dumbbell to rotate. As the dumbbell rotates, the mirror moves with it, changing the angle of reflection of the light beam. This change is detected by the photocell. The output of the photocell is amplified and transmitted to the indicating unit that is calibrated to read out the oxygen percentage.

The oxygen gas analyzer was routinely calibrated during test. The high calibration was conducted by passing air through the analyzer and high calibration setpoint was set to $20.95 \%$. Low calibration was conducted with pure nitrogen and low calibration setpoint was set to $0.00 \%$. The specifications of the sample gas are shown in Fig. 7. Gas sample was taken from the vent panel location.

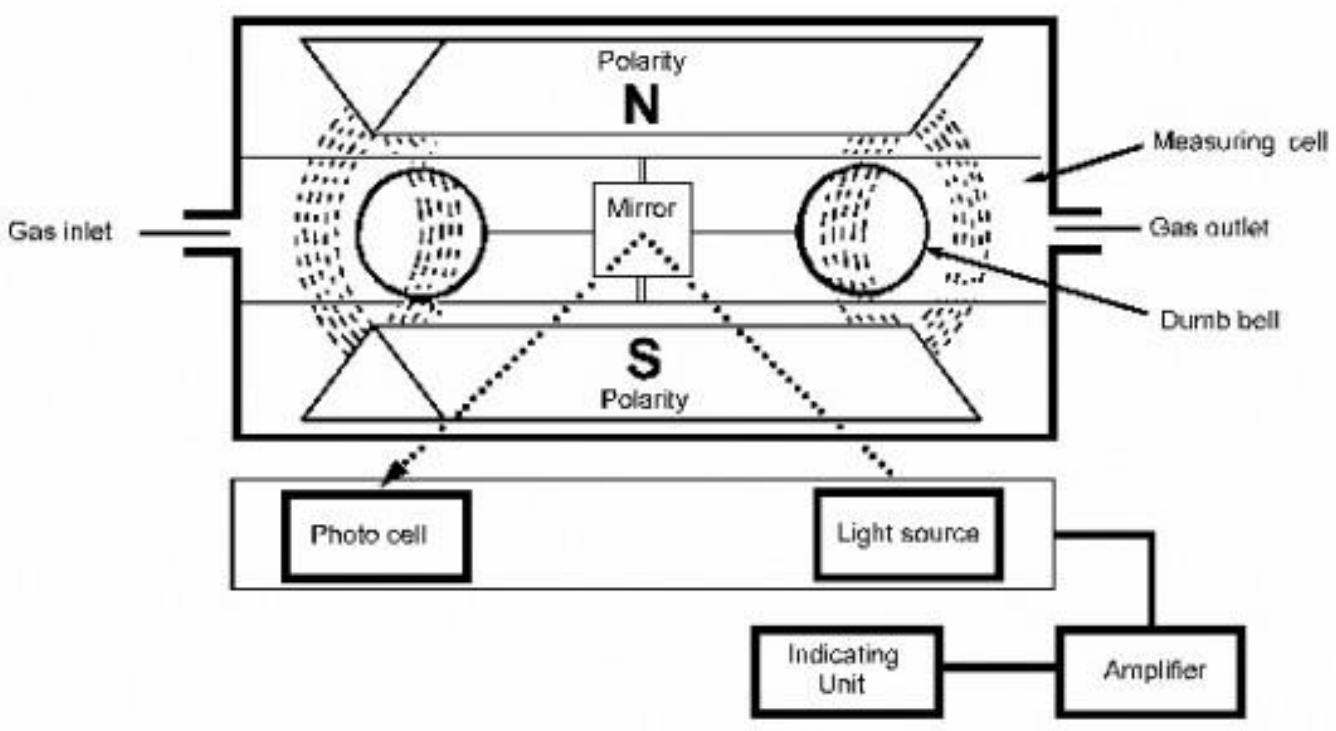

Figure 6: Paramagnetic cell [209]. 


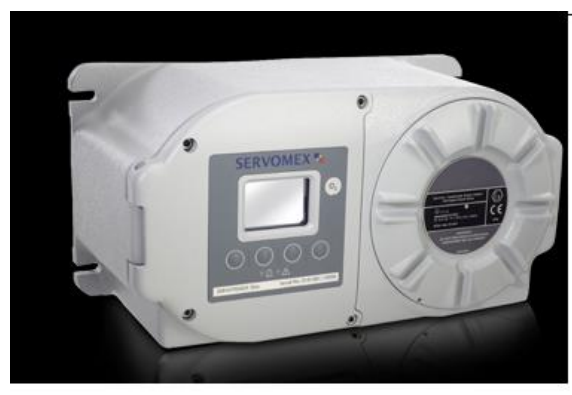

Flow rates

Standard inlet/outlet $\quad 150$ to $250 \mathrm{ml} \mathrm{min}^{-1}\left(200 \mathrm{ml} \mathrm{min}^{-1}\right.$ recommended)

High flow bypass ${ }^{\dagger} \quad 50$ to $70 \mathrm{I} \mathrm{h}^{-1}\left(60 \mathrm{I} \mathrm{h}^{-1}\right.$ recommended)

Maximum inlet pressure

Standard inlet/outlet

$0.2 \mathrm{kPa}(0.03 \mathrm{psi})$ relative to sample vent pressure

High flow bypass ${ }^{\dagger} \quad 1.4 \mathrm{kPa}(0.2 \mathrm{psi})$ relative to sample vent pressure

Figure 7: Servomex SERVOTOUGH Oxy 1900 sample gas specifications.

PXM409-USBH series micro machined silicon piezoresistive pressure transducer were used to record the pressure during each experiment. It was manufactured by embedding strain gauges into a silicon substrate on a molecular level. When force is applied to the substrate material, the resistance of the material changes. The surface of the substrate material is connected to a Wheatstone bridge, which detects the resistance change and reports it as a change in pressure. The sampling rate of a pressure transducer was selected as 1000 updates/seconds for each experiment. The specifications of the pressure transducer are shown in Fig. 8. Due to a low flame speed, using one pressure sensor was found to be sufficient [157]. The pressure transducer was placed on the vent panel location.

\begin{tabular}{|l|l|l|}
\hline \multicolumn{2}{|c|}{ Specifications: } \\
\cline { 2 - 3 } & Accuracy: & $0.08 \% \mathrm{BSL}$ \\
\hline Bandwidth: & $\mathrm{DC}$ to 1000 updates per second \\
\hline $\begin{array}{c}\text { Operating } \\
\text { Temperature } \\
\text { Range }\end{array}$ & -40 to $85^{\circ} \mathrm{C}$ \\
\hline $\begin{array}{c}\text { Operating } \\
\text { Pressure Range }\end{array}$ & $0-1$ bar \\
\hline
\end{tabular}

Figure 8: PXM409-USBH pressure transducer specifications.

A high-speed camera (Chronos, Model 1.4) was used to record the flame evolution inside and outside (in some experiments) of the vessel. Computar brand (M6Z1212-3S) lens was used for the high-speed camera. Images were recorded at a rate of 1057 frames per second and resolution was $1280 \times 1024$. The high-speed camera was positioned perpendicular to the combustion chamber in a way to capture the complete chamber, as shown in Fig. 4. 


\subsubsection{Image Analysis}

Post-processing of recorded videos was conducted to tabulate the time evolutions of the flame tip position and its velocity, of the flame surface area, and of the unburned gas volume. During the video analysis, Matlab and ImageJ [210] software were used.

For central ignition experiments, ImageJ software was used for image analysis. A simple process diagram of ImageJ processing is shown in Fig. 9. In order to achieve accurate results and highquality of flame images, the following processes were used. (i-ii) An image to be analyzed is converted into gray scale. (iii) A Sobel edge detector was used to highlight sharp edges/gradients. (iv) In order to take the flame to the foreground, background and foreground colors were switched. (v) To reveal the flame with a better resolution, brightness and contrast degrees were adjusted. (vi) Finally, noise was removed.

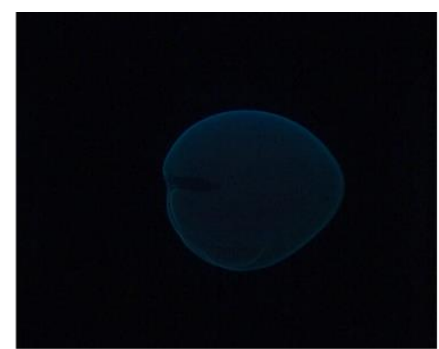

(i) Original image

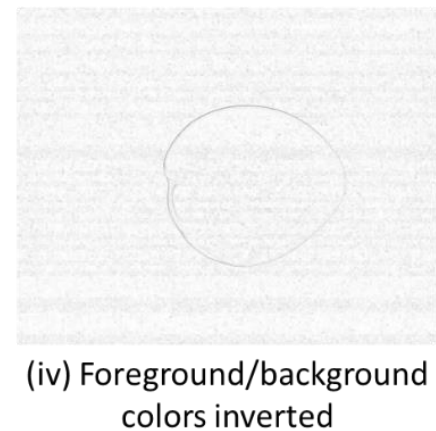

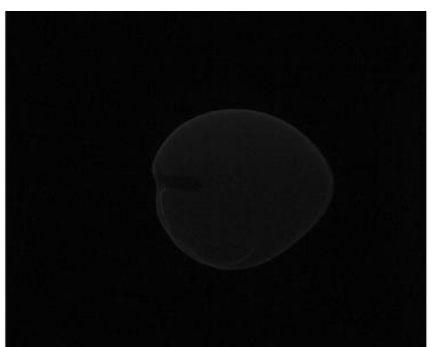

(ii) Gray scale

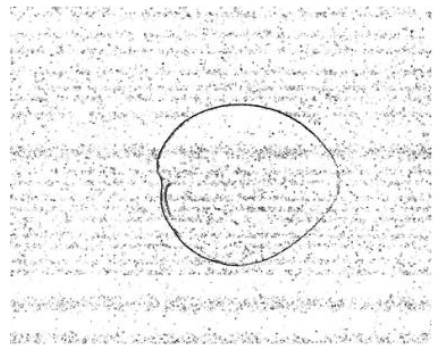

(v) Brightness/contrast adjusted

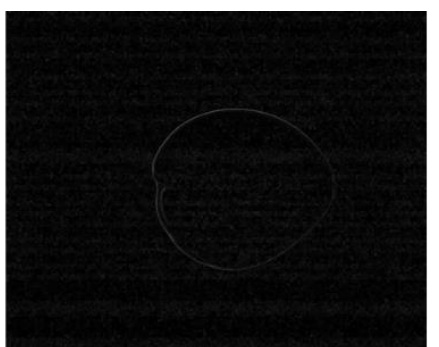

(iii) Edge detection

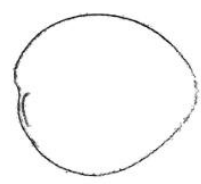

(vi) Noises removed

Figure 9: Image analysis of videos in ImageJ.

For rear ignition experiments, two Matlab codes were developed and used for the video analysis. Specifically, one code was written to obtain the flame tip position and its velocity. The code read each frame of the video. Ignition location was manually entered in this code for each video. The flame tip position was detected by scanning each column of the ignition row for a threshold blue pixel intensity. This scan was completed reversely, i.e. from the vent panel side to ignition side, to minimize the number of misdetections. The detected flame tip position data (in pixel units) was 
then processed and converted into the actual dimensions. The second code was developed to calculate the flame surface area and burned gas volume. For this purpose, the ignition location was manually entered in the code for each video. The evolving flame surface was captured by scanning each row in each column of the ignition row for a threshold blue pixel intensity. Such a scanning was conducted both from upside and downside, reversely from the dark side to the flame, to minimize the number of misdetections and to detect the flame surface both upside and downside for each column of the ignition row. An example of such a flame surface detection is shown in Fig. 10.

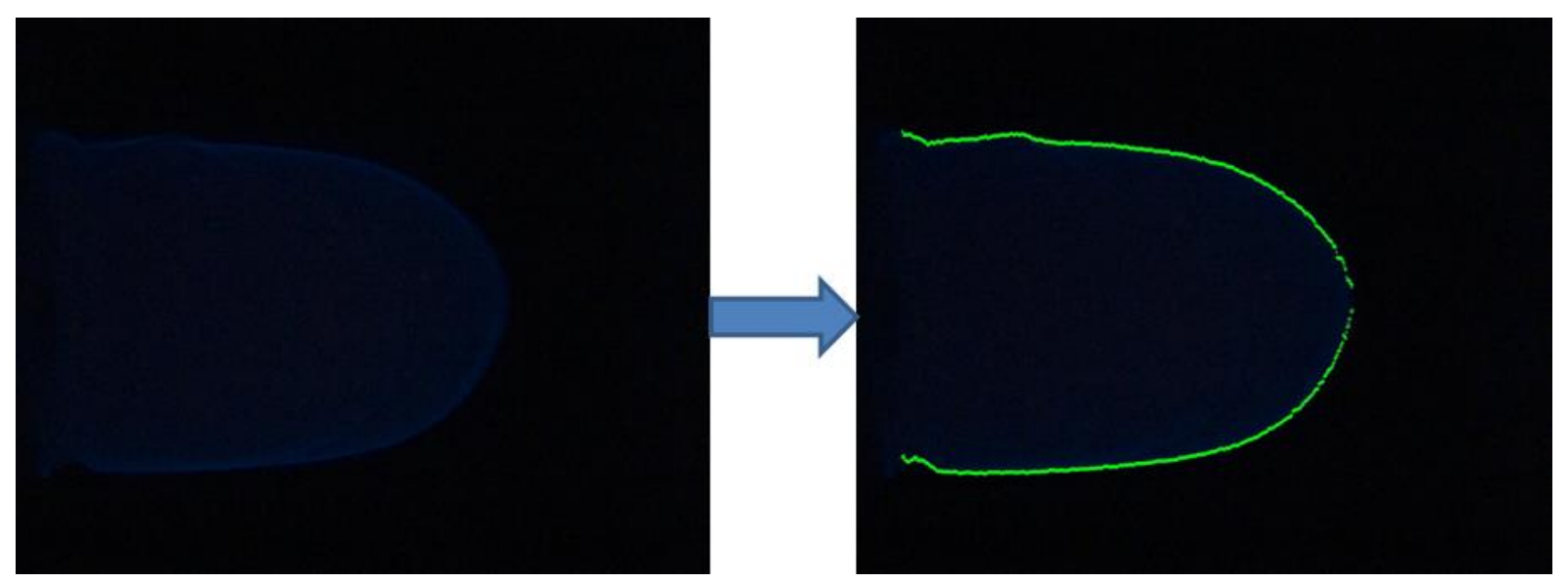

Figure 10: Flame surface detection.

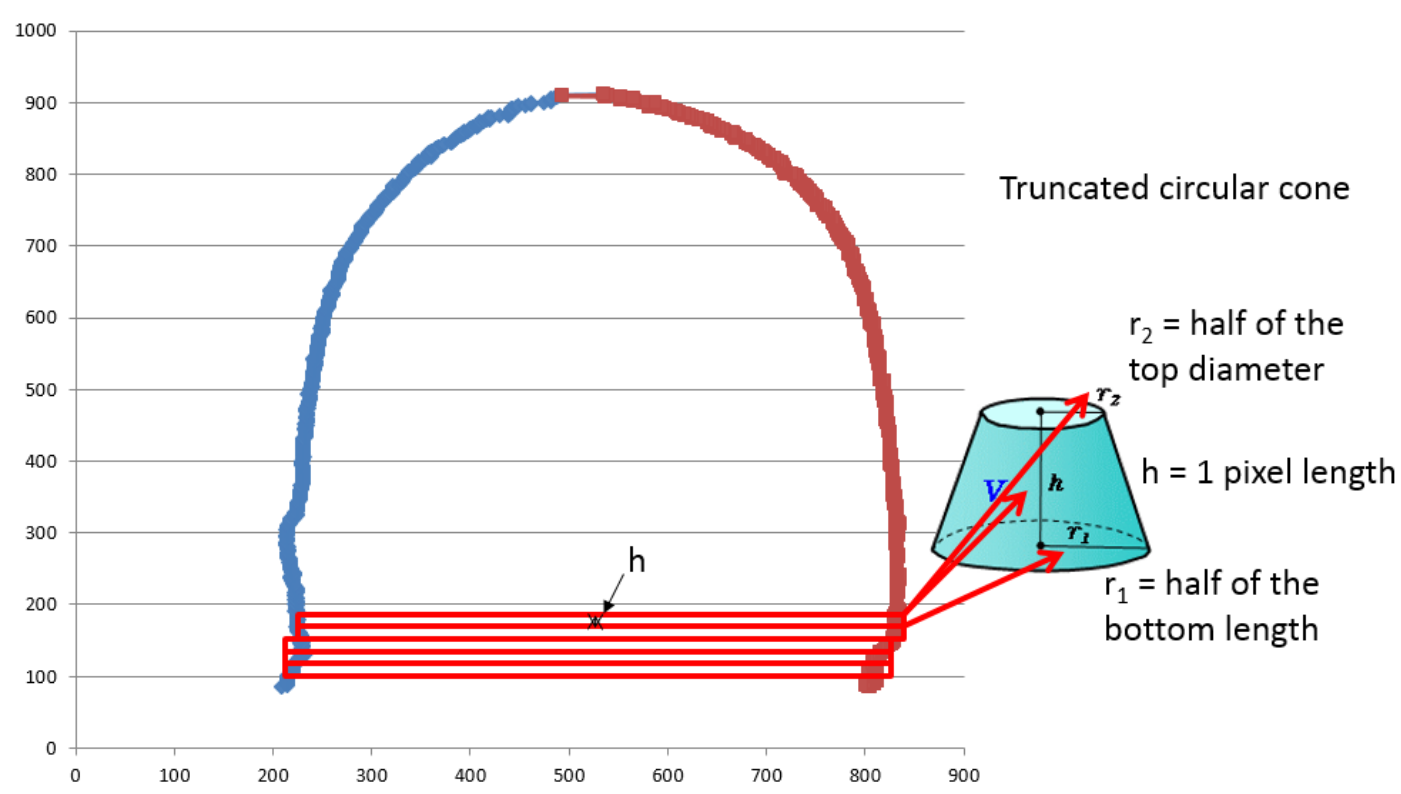

Figure 11: Truncated circular cone method applied to the flame surface. 
After detecting the flame surface in each frame, the "truncated circular cone" model [211] was applied to each frame to calculate the surface area and the volume. This model is illustrated in Fig. 11. The pixel length was known before each experiment. The top and the bottom diameters of the truncated circular zone for each pixel distance was calculated by the program. In fact, it was enough to calculate the surface area and volume of the cone between two neighboring pixels. The surface area of the flame and burned gas volume then became the accumulative calculation of the surface area of each two neighboring pixels, along with the burned gas volume. This process was conducted for each frame of each experiments.

\subsubsection{Single Cylinder Experiments}

Schematic of the experimental setup with a single cylinder of length $30 \mathrm{~cm}$ is shown in Fig. 12. A parametric study was performed by varying the vent size and the ignition location. Specifically, the ignition was triggered in the center and rear of the cylinder. Moreover, small $\left(67.9 \mathrm{~cm}^{2}\right)$, medium $\left(86.6 \mathrm{~cm}^{2}\right)$ and large $\left(132.7 \mathrm{~cm}^{2}\right)$ vents were used to analyze the effect of vent size on the pressure development inside the cylinder during the combustion process.

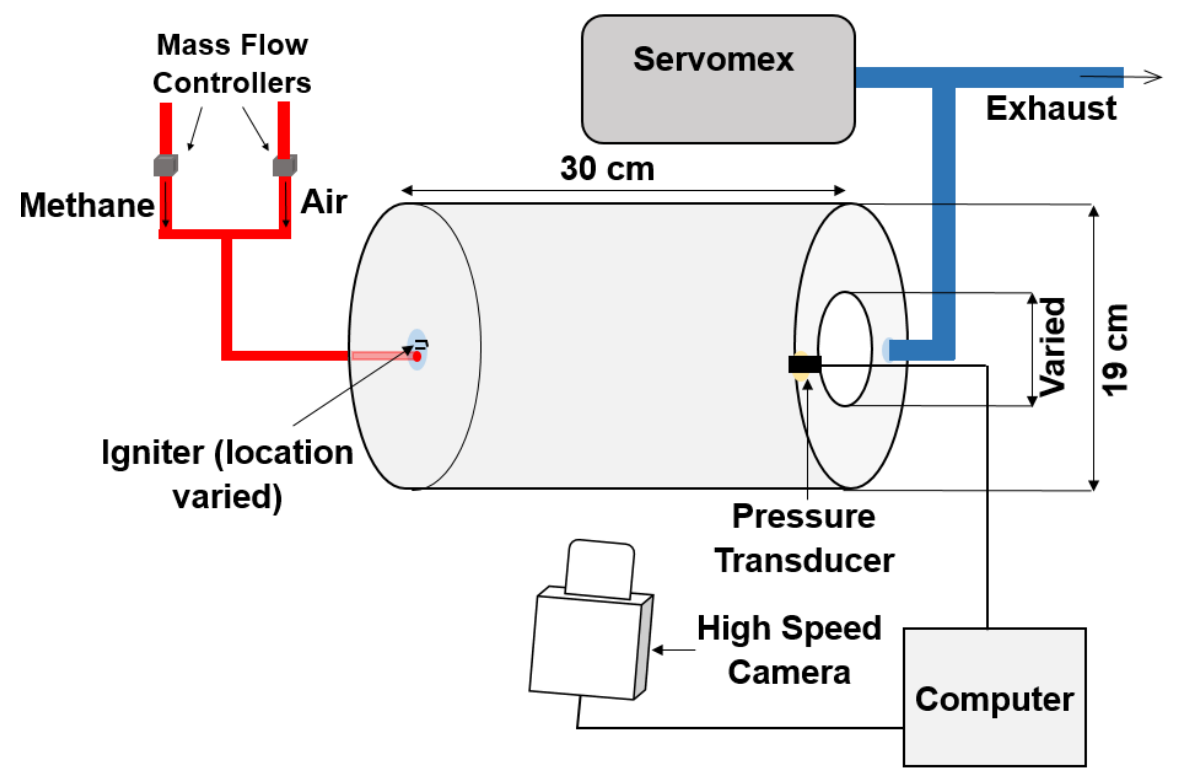

Figure 12: Schematic of the single cylinder experimental setup.

Figure 13 presents evolutions of pressure (a) and the pressure rate (b), during explosions of lean $(\phi=0.8)$, stoichiometric $(\phi=1)$, and rich $(\phi=1.2)$ methane-air mixtures in the single-cylinder configuration with a small vent $\left(67.9 \mathrm{~cm}^{2}\right)$ and center ignition. The solid line of each case shows 
the average value of three repeated experiments and the shaded area of each case represents the experimental error. It is seen that the maximum pressure development was observed in the stoichiometric case as $P_{\max }=0.019$ bar-g. This was followed by the fuel-rich, methane-air explosion with $P_{\max }=0.013$ bar-g and by the fuel-lean methane-air mixture explosion with $P_{\text {max }}=0.011$ bar-g. The maximum rate of pressure rise was observed in the stoichiometric case $(d P / d t)_{\max }=2.482$ bar-g/s and was followed by fuel-rich and fuel-lean methane-air conditions as 1.552 bar-g/s and 1.149 bar-g/s, respectively.
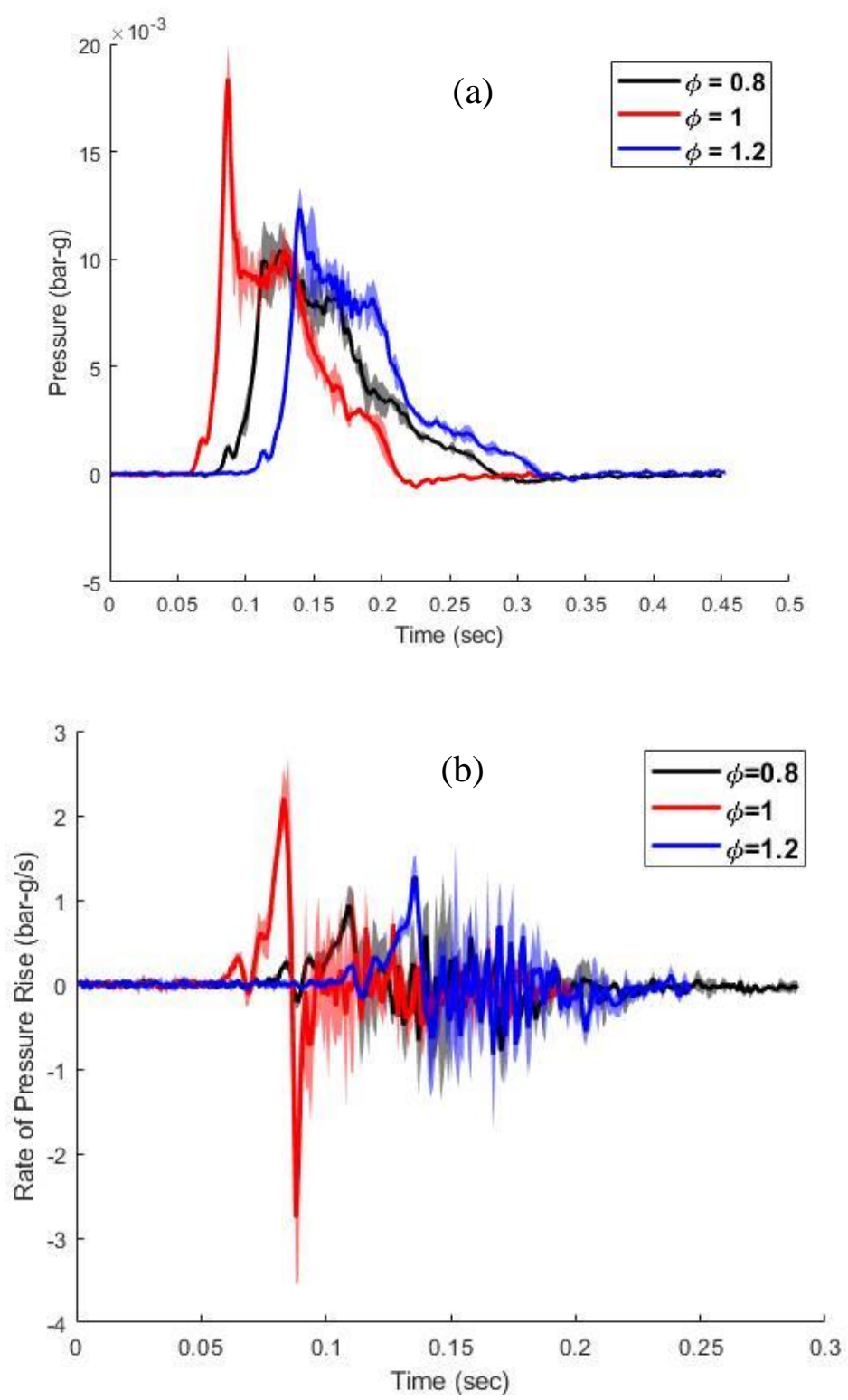

Figure 13: Time evolution of (a) pressure and (b) rate of pressure rise of lean $(\phi=0.8)$, stoichiometric $(\phi=1)$ and rich $(\phi=1.2) \mathrm{CH}_{4}$-air explosions in a single cylinder with small vent and center ignition. 

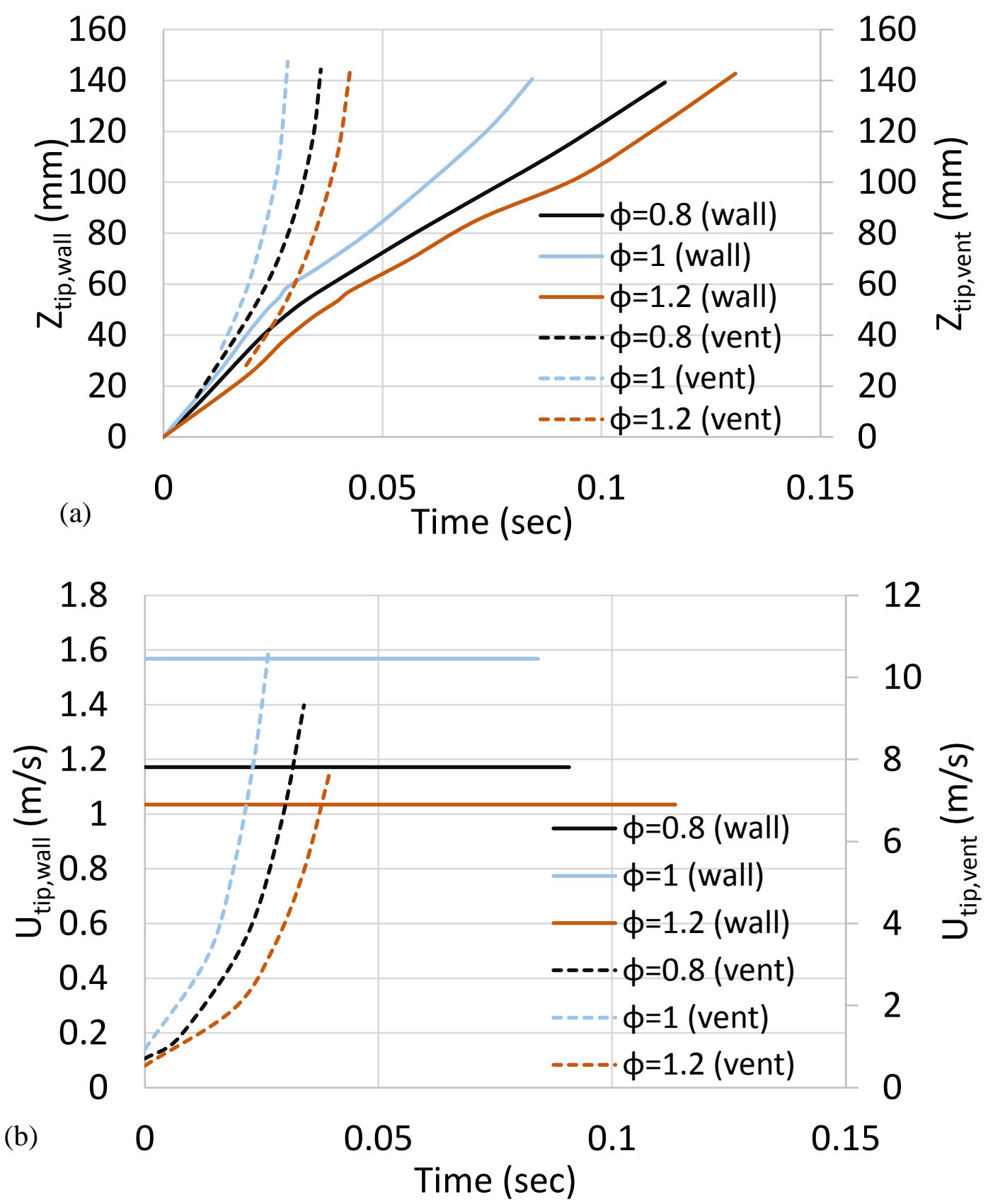

Figure 14: Time evolution of the flame tip position (a) and velocity (b) towards wall and vent of the cylinder of lean $(\phi=0.8)$, stoichiometric $(\phi=1)$ and rich $(\phi=1.2)$ methane-air explosions in a single cylinder with small vent and center ignition.

Figure 14 shows the evolutions of flame tip position (a) and velocity (b) towards wall and vent directions of a single-cylinder with central ignition. Again, three $\mathrm{CH}_{4}$-air compositions, lean $(\phi=$ $0.8)$, stoichiometric, and rich $(\phi=1.2)$ were considered. It is seen that the flame towards the vent 
accelerated exponentially in all the cases. The exponential factor $\sigma$ of the form $\propto \exp (\sigma t)$ is plotted versus the equivalence ratio in Fig. 15.

Fastest acceleration towards vent was found in the stoichiometric case as $\sigma=92.1 \mathrm{~s}^{-1}$ and this was followed by fuel-lean $\phi=0.8$ and fuel-rich $\phi=1.2$ methane air mixtures as $\sigma=75.7 \mathrm{~s}^{-1}$ and $67.5 \mathrm{~s}^{-1}$, respectively. On the other hand, the flame propagated towards the wall with a constant velocity due to the interplay of the "push" and "pull" effects of flame thermal expansion and the venting force backwards. The velocities of the methane-air flames towards vent were calculated as $1.2,1.6$ and $1 \mathrm{~m} / \mathrm{s}$ for $\phi=0.8,1$ and 1.2 respectively.

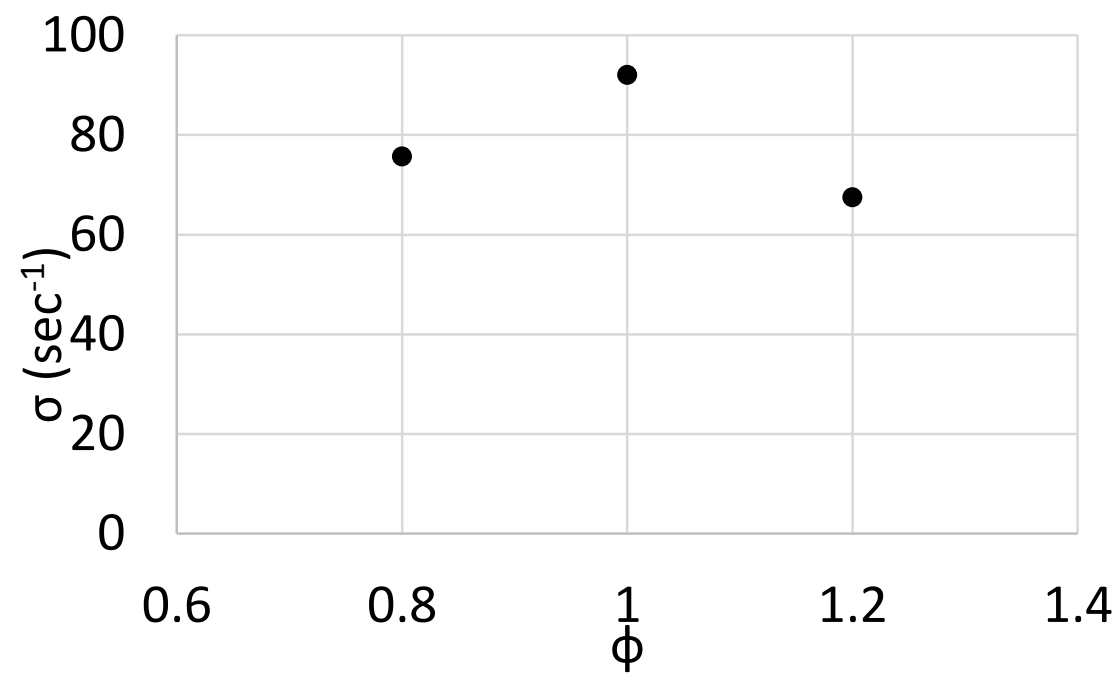

Figure 15: The exponential factor $\sigma$ versus the equivalence ratio $\phi$ in a single-cylinder with central ignition and small $\left(67.9 \mathrm{~cm}^{2}\right)$ vent.

Figure 16 shows the evolutions of pressure and of the rate of pressure rise of $\phi=0.8,1$ and 1.2 methane-air combustion in a single-cylinder with medium vent $\left(86.6 \mathrm{~cm}^{2}\right)$ and central ignition. Specifically, Fig. 16a shows the pressure history whereas Fig. 16b presents the evolution of the rate of pressure rise. It is seen here that shaded areas are larger, indicating that the experimental repeatability is worse as compared to Fig. 13 (small vent and center ignition). The highest pressure was obtained in the stoichiometric case as $P_{\max }=0.012$ bar-g. Contrary to the small vent condition, here the lean mixture of $\phi=0.8$ created the maximum pressure of $P_{\max }=0.01 \mathrm{bar}-\mathrm{g}$, which was twice higher than $P_{\max }=0.005$ bar-g for the rich mixture of $\phi=1.2$. The maximum rate of pressure rise was observed in the case of stoichiometric burning too $(d P / d t)_{\max }=2.117$ bar-g/s, followed by $1.329 \mathrm{bar}-\mathrm{g} / \mathrm{s}$ for $\phi=0.8$ and $0.546 \mathrm{bar}-\mathrm{g} / \mathrm{s}$ for $\phi=1.2$, respectively. 

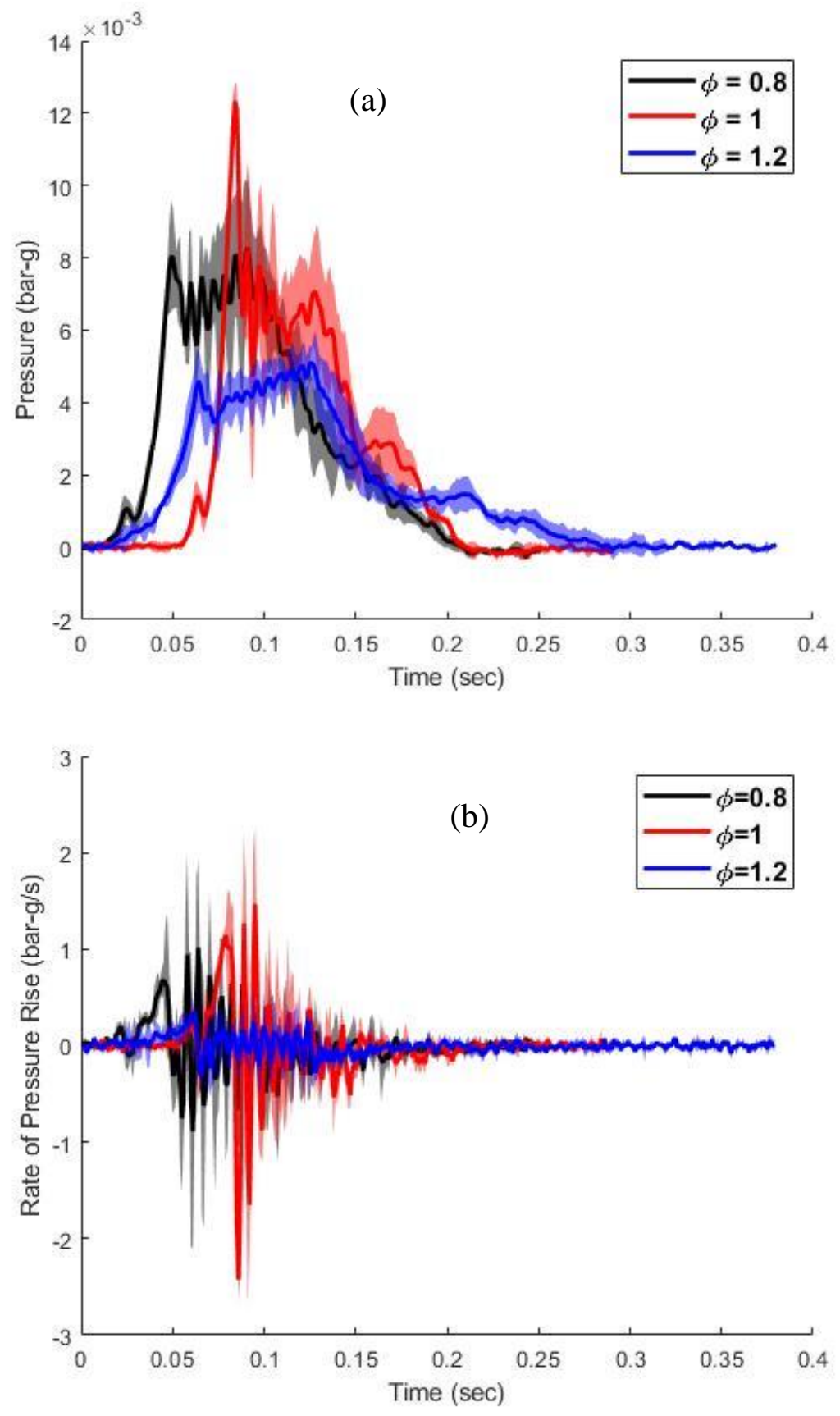

Figure 16: The time evolution of pressure (a) and of the rate of pressure rise (b) for lean $(\phi=0.8)$, stoichiometric $(\phi=1)$ and rich $(\phi=1.2)$ methane-air burning in a single cylinder with medium vent area $\left(86.6 \mathrm{~cm}^{2}\right)$ and central ignition. 

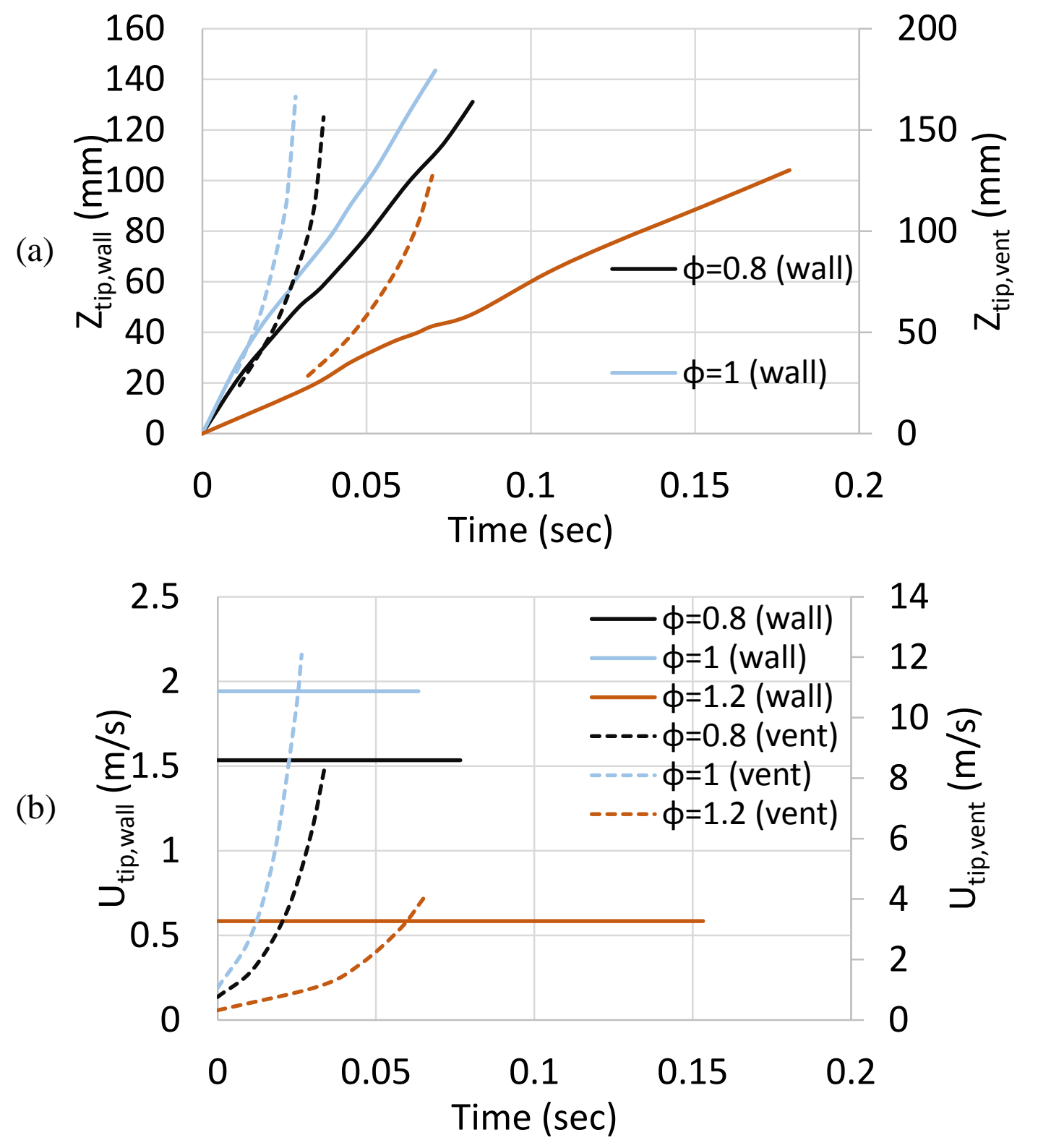

Figure 17: Time evolution of the flame tip position (a) and velocity (b) towards wall and vent directions of lean $(\phi=0.8)$, stoichiometric $(\phi=1)$, and rich $(\phi=1.2)$ methane-air flames in a single cylinder with medium vent area $\left(86.6 \mathrm{~cm}^{2}\right)$ and central ignition.

Figure 17 shows the corresponding flame tip positions and velocities versus time for the lean, stoichiometric, and rich methane-air flames towards wall and vent directions. Figure 18 presents the exponential acceleration factor $\sigma$ versus the equivalence ratio $\phi$. It is seen that the flame in the medium $\left(86.6 \mathrm{~cm}^{2}\right)$ vent configuration accelerated exponentially towards vent as well and fastest flame acceleration was observed in the stoichiometric case $\phi=1$ with the acceleration rate $\sigma$ being $91.7 \mathrm{~s}^{-1}$. Similar to the small $\left(67.9 \mathrm{~cm}^{2}\right)$ vent configuration results, this was followed by the 
lean, $\phi=0.8$, and rich, $\phi=1.2$, conditions, with the exponential factor $\sigma$ being $70.8 \mathrm{~s}^{-1}$ and $39 \mathrm{~s}^{-}$ ${ }^{1}$, respectively. Flame towards the wall, similar to the small $\left(67.9 \mathrm{~cm}^{2}\right)$ vent configuration, moved in the wall direction with constant velocity. Fastest burning occurred at the stoichiometric condition, followed by the $\phi=0.8$ and $\phi=1.2$ cases, with the velocities $U_{\text {tip,wall }}=1.9,1.5$ and $0.6 \mathrm{~m} / \mathrm{s}$.

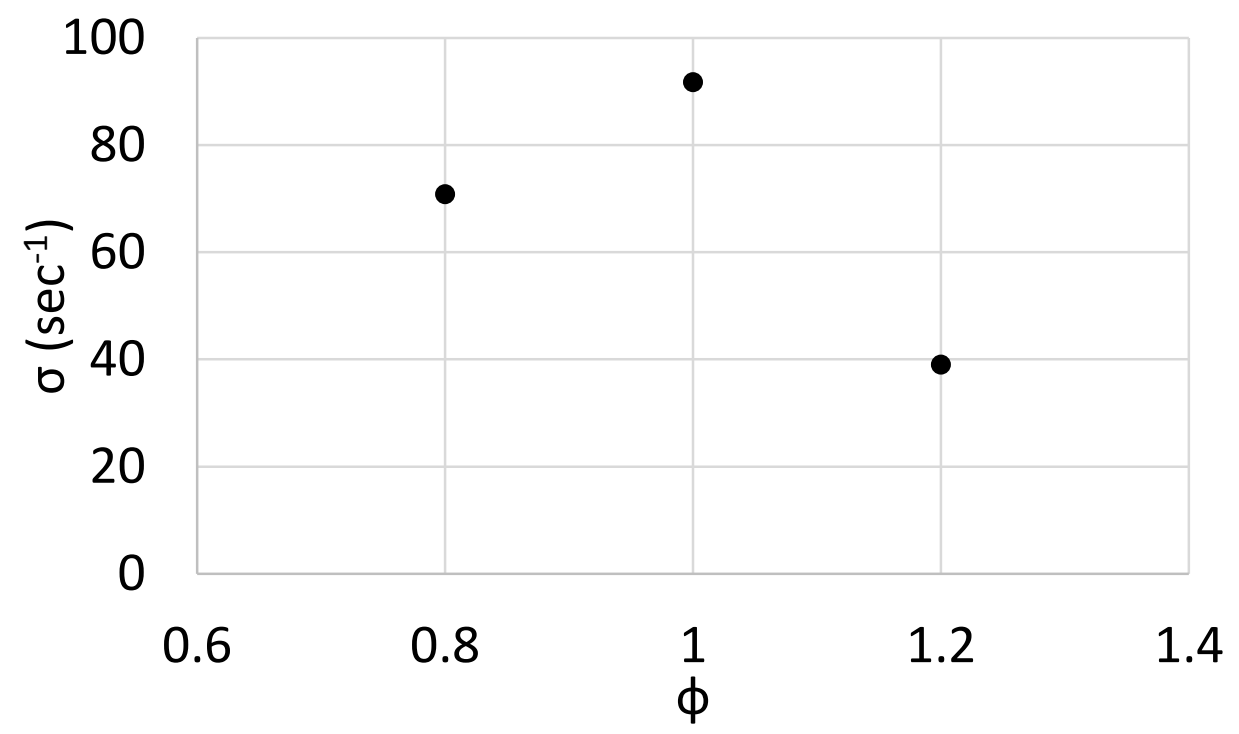

Figure 18: Exponential factor $\sigma$ versus equivalence ratio in a single cylinder with central ignition and medium $\left(86.6 \mathrm{~cm}^{2}\right)$ vent.

Figure 19 shows the evolution of pressure (a) and that of the rate of pressure rise (b) for $\phi=0.8$, 1 , and 1.2 methane-air explosions in a single cylinder of length $30 \mathrm{~cm}$ with a large $\left(132.7 \mathrm{~cm}^{2}\right)$ vent area and central ignition. It is seen that the highest maximum pressure of $P_{\max }=0.006$ bar$\mathrm{g}$ was observed in the stoichiometric case. Similar to the small $\left(67.9 \mathrm{~cm}^{2}\right)$ vent but contrary to the medium $\left(86.6 \mathrm{~cm}^{2}\right)$ vent configurations, here rich $(\phi=1.2)$ methane-air explosion created a higher maximum pressure, $P_{\max }=0.005$ bar-g, than the lean $(\phi=0.8)$ one, where the maximum pressure was measured as $P_{\max }=0.003 \mathrm{bar}-\mathrm{g}$. Moreover, the highest maximum rate of pressure rise was seen in the case of $\phi=1.2$, being $(d P / d t)_{\max }=0.855 \mathrm{bar}-\mathrm{g} / \mathrm{s}$. This is contrary to both small $\left(67.9 \mathrm{~cm}^{2}\right)$ and medium $\left(86.6 \mathrm{~cm}^{2}\right)$ vent configurations where the highest maximum rate of pressure rise $(d P / d t)_{\max }$ was observed in the stoichiometric case. The second highest maximum rate of pressure rise of $(d P / d t)_{\max }=0.744 \mathrm{bar}-\mathrm{g} / \mathrm{s}$ was observed in the stoichiometric case for the large $\left(132.7 \mathrm{~cm}^{2}\right)$ vent configuration, and this was followed by lean $(\phi=0.8)$ methane-air explosion as $(d P / d t)_{\max }=0.311 \mathrm{bar}-\mathrm{g} / \mathrm{s}$. 
Figure 20 presents the evolution of the flame tip position, Fig. 20a, and velocity, Fig. 20b, of the $\phi=0.8,1$ and 1.2 methane-air explosions towards both wall and vent directions. Similar to small $\left(67.9 \mathrm{~cm}^{2}\right)$ and medium $\left(86.6 \mathrm{~cm}^{2}\right)$ vent configurations, two regimes of flame propagation were observed in the large $\left(132.7 \mathrm{~cm}^{2}\right)$ vent as well, namely: (i) linear flame movement towards the wall and (ii) exponential acceleration of flame towards vent direction.
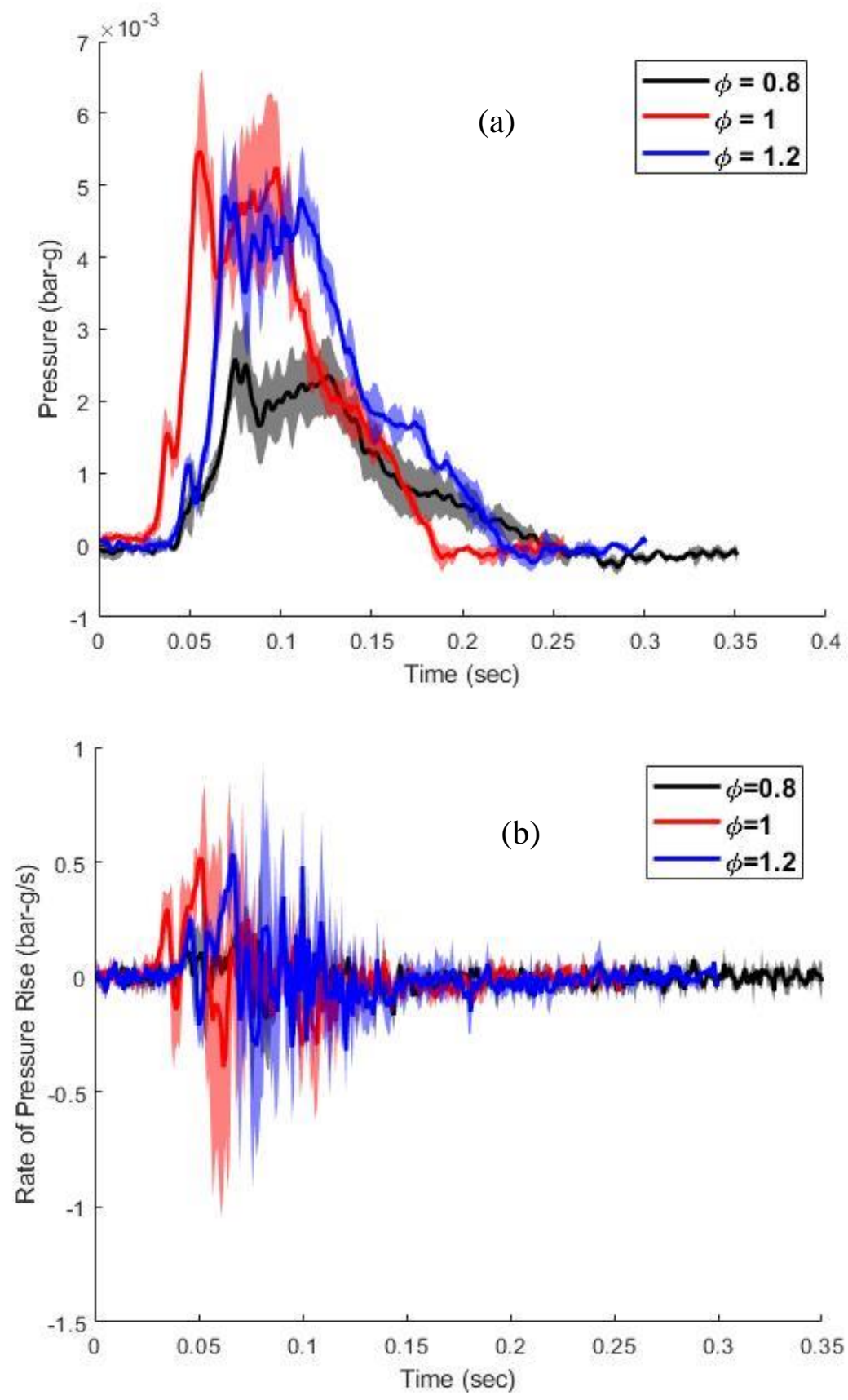

Figure 19: Time evolution of pressure (a) and of the rate of pressure rise (b) of rich $(\phi=1.2)$, stoichiometric $(\phi=1)$ and lean $\phi=0.8$ methane-air explosions in a single cylinder with a large (132.7 $\mathrm{cm}^{2}$ ) vent area and central ignition. 


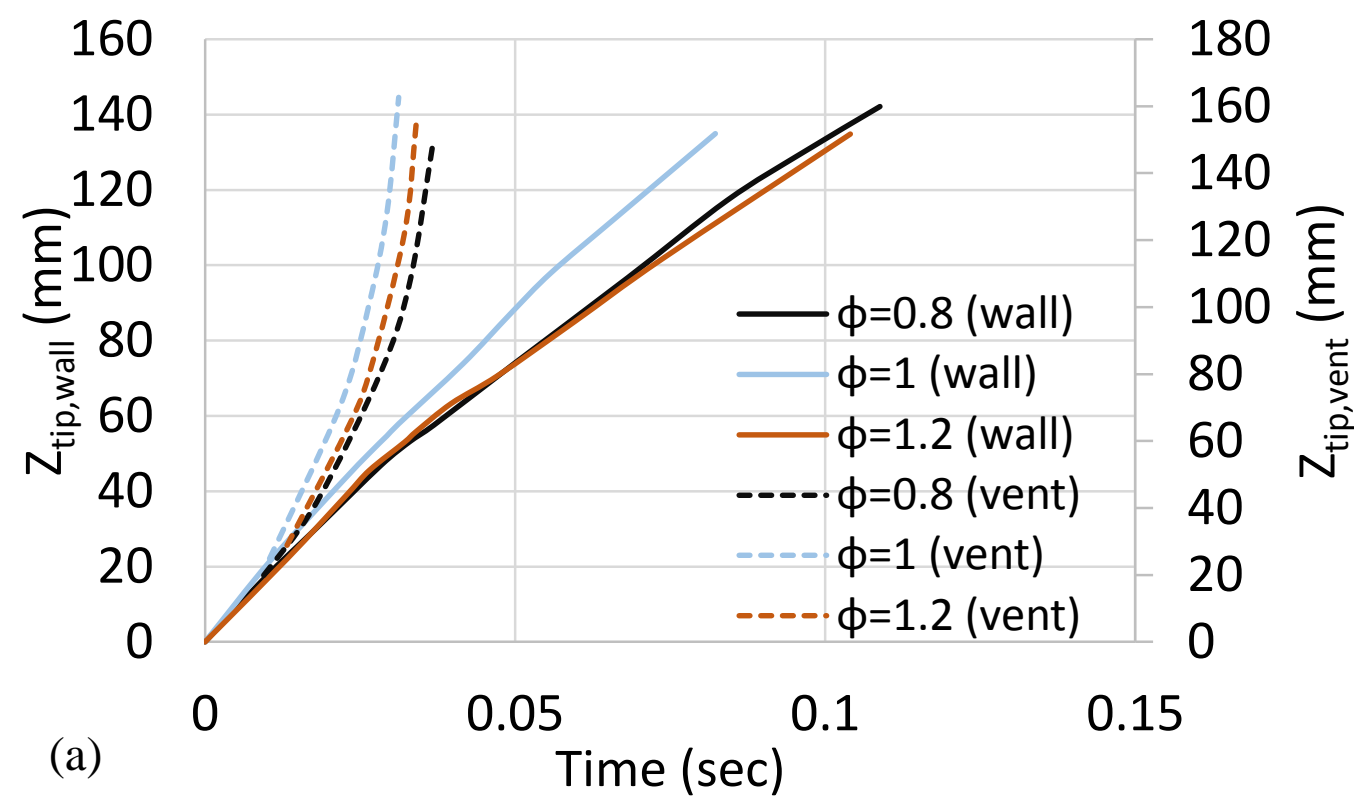

(b)

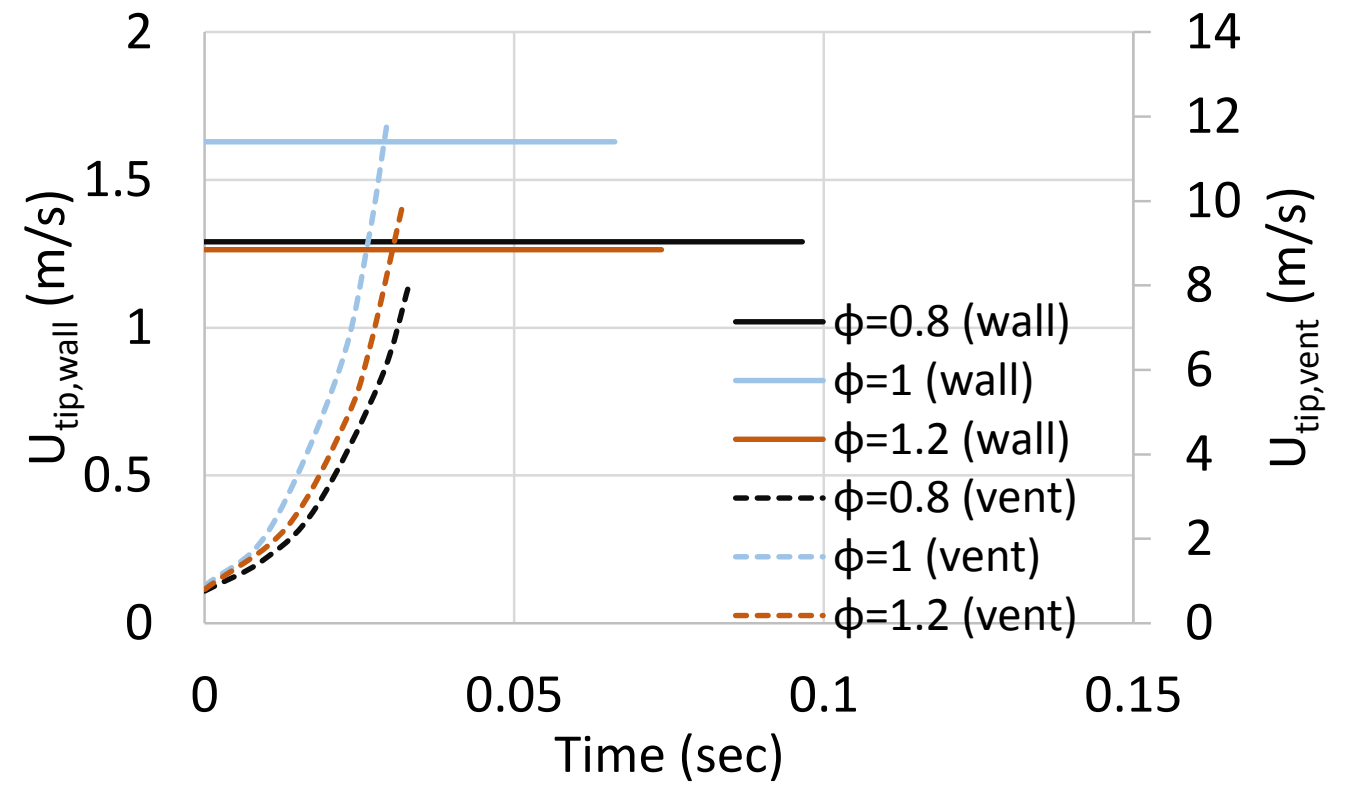

Figure 20: Time evolution of the flame tip position (a) and velocity (b) towards the wall and the vent for the lean $(\phi=0.8)$, stoichiometric $(\phi=1)$ and rich $(\phi=1.2)$ methane-air explosions in a single cylinder with large $\left(132.7 \mathrm{~cm}^{2}\right)$ vent area and central ignition.

Figure 21 presents the exponential acceleration rate $\sigma$ obtained from the trend of the flame tip velocity in the vent direction $U_{\text {tip,vent }}$ in Fig. 20b. It is seen that the highest exponential factor $\sigma$, $\sigma=88.3 \mathrm{~s}^{-1}$ was obtained in the stoichiometric case; followed by $\sigma=78.5 \mathrm{~s}^{-1}$ for $\phi=1.2$ and $\sigma=71.4 \mathrm{~s}^{-1}$ for $\phi=0.8$. Fastest flame motion towards the wall was observed in the stoichiometric case, with a constant velocity of $U_{\text {tip,wall }}=1.6 \mathrm{~m} / \mathrm{s}$. Similar to the small $\left(67.9 \mathrm{~cm}^{2}\right)$ and medium 
$\left(86.6 \mathrm{~cm}^{2}\right)$ vent, the fastest flame motion towards the wall was followed by that for $\phi=0.8$ and $\phi=1.2$, respectively, with almost equal velocities of $U_{t i p, \text { wall }} \cong 1.3 \mathrm{~m} / \mathrm{s}$.

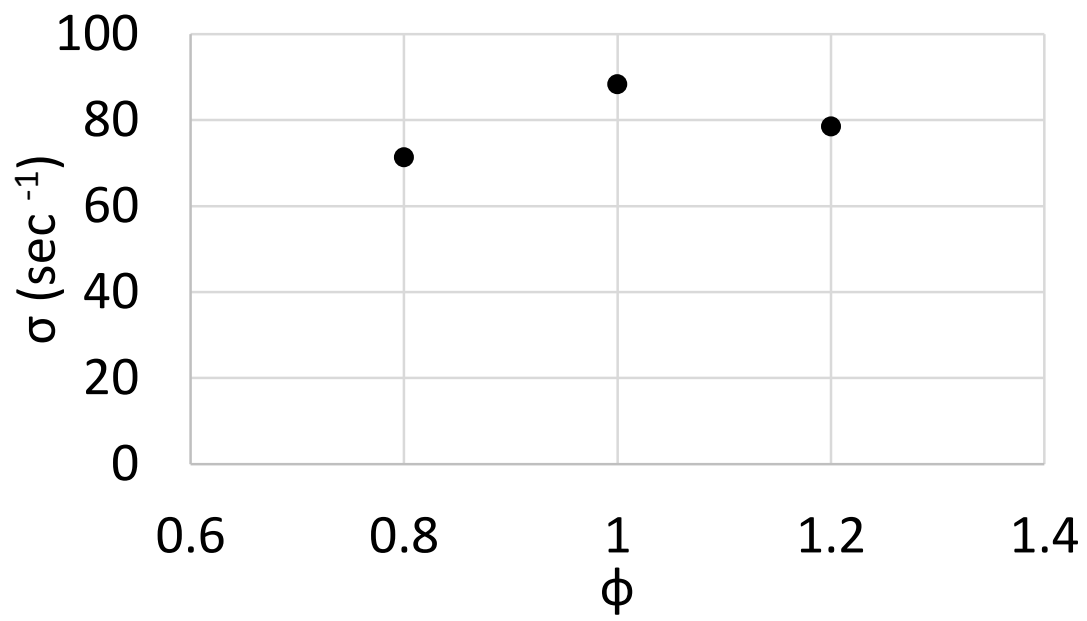

Figure 21: The exponential factor $\sigma$ versus equivalence ratio $\phi$ in a single cylinder with large $\left(132.7 \mathrm{~cm}^{2}\right)$ vent area and central ignition.

In the rich $(\phi=1.2)$ methane-air explosions in a single cylinder, a flashback phenomenon occurred in the recorded videos at the times around $t \approx 150$ and $180 \mathrm{~ms}$, respectively for vent areas of $A_{v}=86.6$ and $132.7 \mathrm{~cm}^{2}$, and similarly at $t \approx 240 \mathrm{~ms}$, for the vent area of $A_{v}=67.9$ $\mathrm{cm}^{2}$. The flame bounced back from external of the cylinder into the chamber. In Fig. 22, this event is shown for the vent size of $A_{v}=86.6$. The white dash lines in Figs. 22a and 22c show the vessel walls (bottom and left) as well as the vent (two shorter lines in the middle). To be more specific, Fig. 22a presents the initial stage of explosion, when the flame spreads inside the vessel. It is seen here that the embryonic flame initially expanded spherically, but then its right side was predominantly drawn towards the vent such that the overall flame front acquired an elongated, ellipsoidal-like shape. Figure 22b is a sequel of Fig. 22a, showing flame propagation outside the vessel. The amount of remaining fresh gas inside the vessel reduced through the burning process, so the venting process diminishes thereby moderating burning outside the vessel gradually. Further, the external flame came back into the vessel to continue burning the fresh mixture, which was left inside. Such a flashback process is depicted by the snapshots of Fig. 22c. 
a)
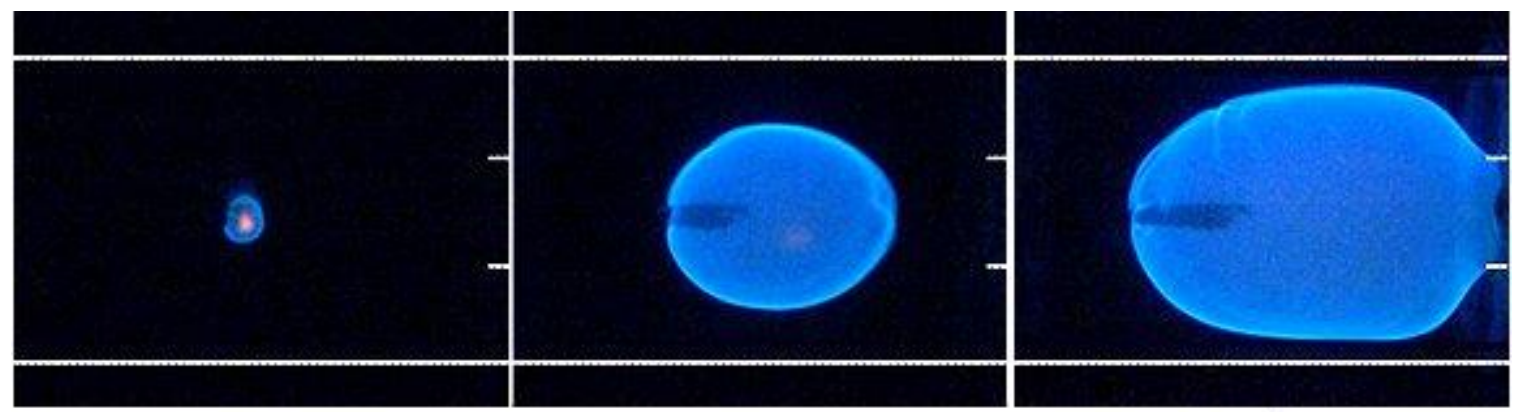

$\mathrm{t}=9 \mathrm{~ms}$

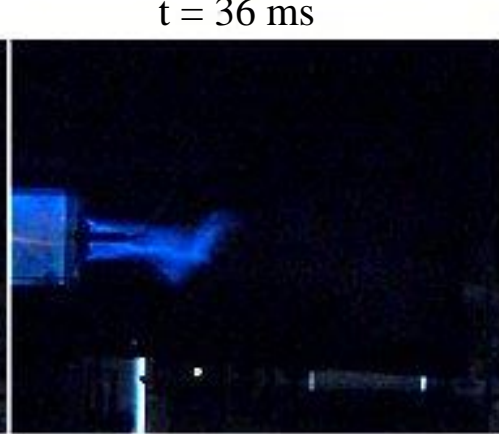

$\mathrm{t}=64 \mathrm{~ms}$

b)

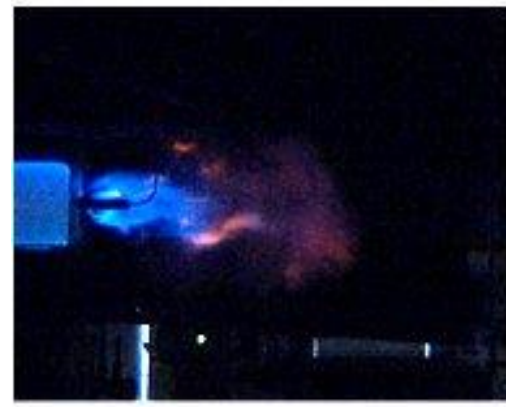

$\mathrm{t}=77 \mathrm{~ms}$

$\mathrm{t}=102 \mathrm{~ms}$

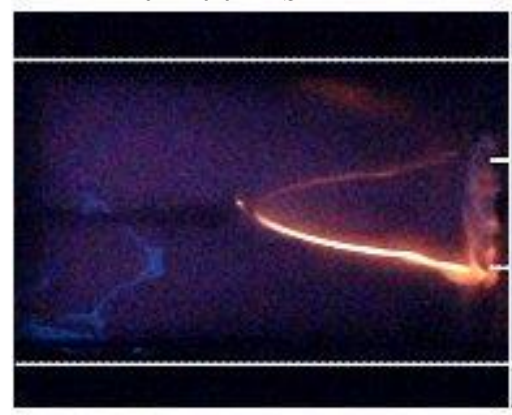

$\mathrm{t}=230 \mathrm{~ms}$

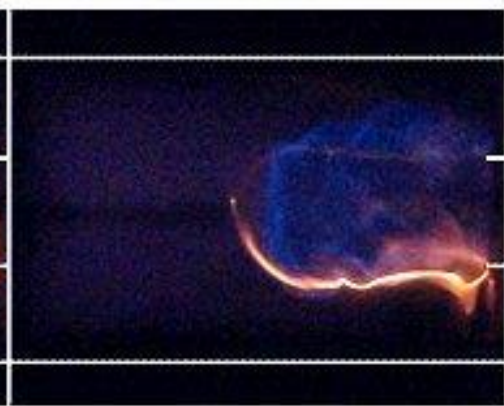

$\mathrm{t}=251 \mathrm{~ms}$

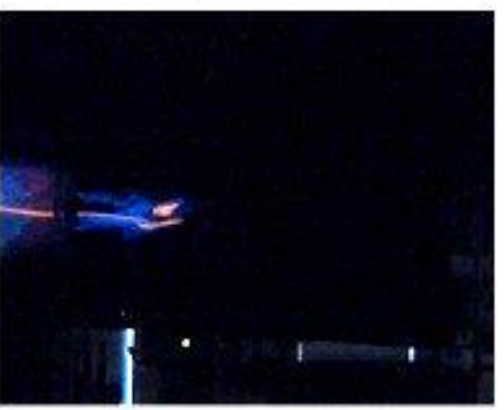

$\mathrm{t}=149 \mathrm{~ms}$

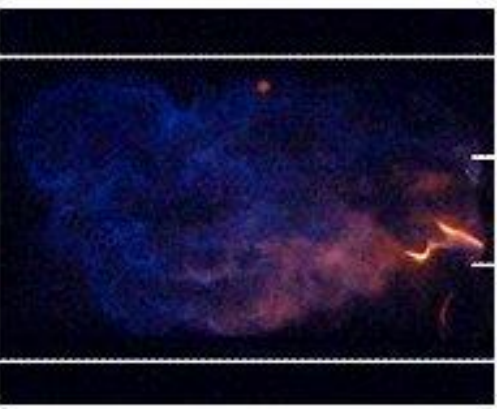

$\mathrm{t}=288 \mathrm{~ms}$

Figure 22: Overall scenario of centrally-ignited rich $(\phi=1.2)$ methane-air explosions both inside and outside the cylinder. a) Initial flame expansion inside the cylinder, b) the flame overshoot and c) flashback to inside of the cylinder.

Figure 23 presents the evolution of the pressure, Fig. 23a, and that of the rate of pressure rise, Fig. $23 \mathrm{~b}$, of $\phi=0.8,1$ and 1.2 methane-air explosions in a single cylinder with a small $\left(67.9 \mathrm{~cm}^{2}\right)$ vent area and rear ignition. Similar to the case of central ignition with the same small $\left(67.9 \mathrm{~cm}^{2}\right)$ vent, the highest maximum pressure of $P_{\max }=0.032$ bar-g corresponded to the stoichiometric explosion; followed by $P_{\max }=0.025$ bar-g for $\phi=1.2$ and $P_{\max }=0.019$ bar-g for $\phi=0.8$. Similar to central ignition with a small $\left(67.9 \mathrm{~cm}^{2}\right)$ vent, the highest maximum rate of pressure rise, $(d P / d t)_{\max }=2.43 \mathrm{bar}-\mathrm{g} / \mathrm{s}$, was observed in the stoichiometric case; followed by $(d P / d t)_{\max }=$ $1.77 \mathrm{bar}-\mathrm{g} / \mathrm{s}$ for $\phi=1.2$ and $(d P / d t)_{\max }=1.224 \mathrm{bar}-\mathrm{g} / \mathrm{s}$ for $\phi=0.8$, respectively. 

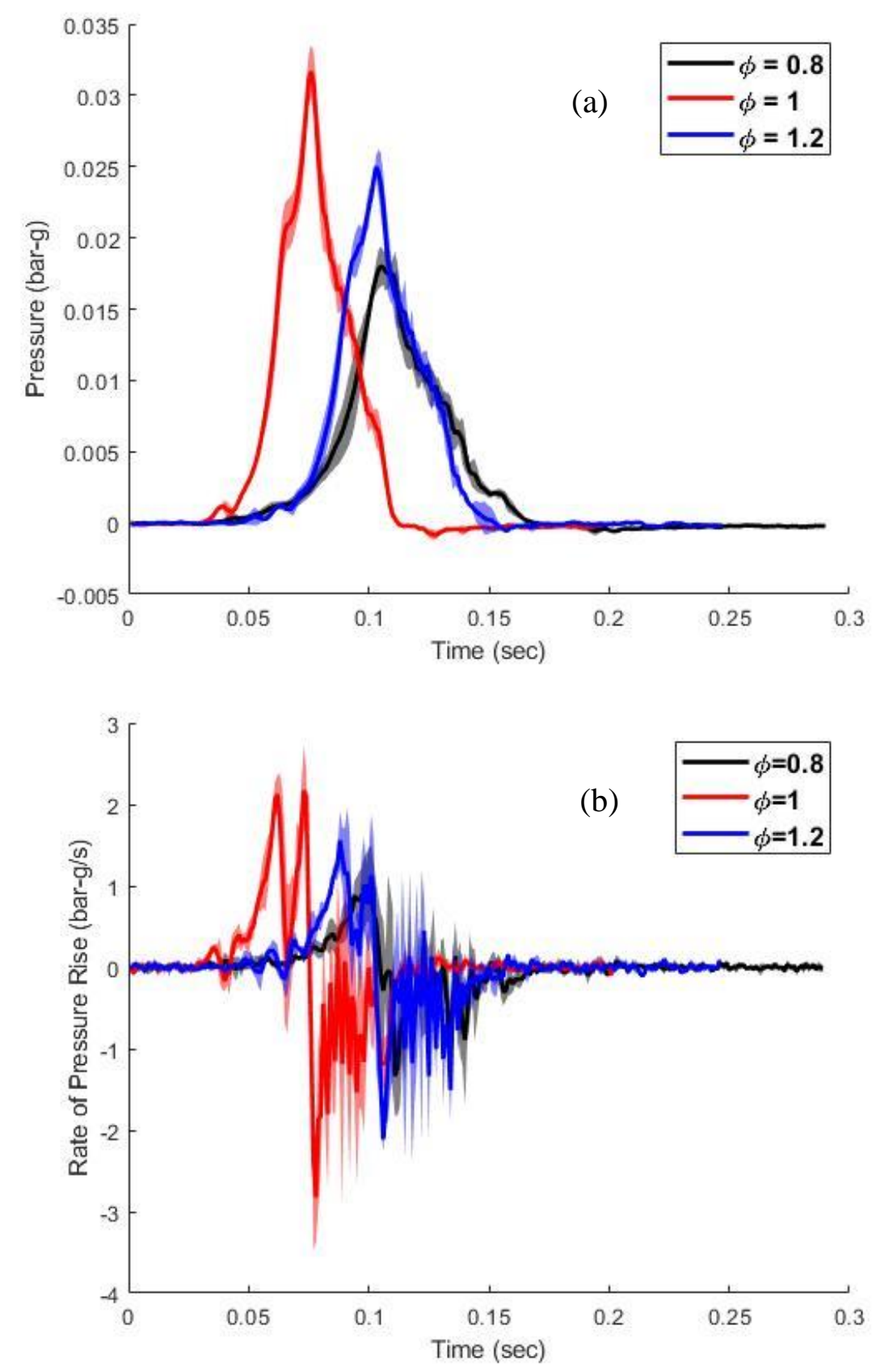

Figure 23: Time evolution of pressure (a) and that of the rate of pressure rise (b) for the lean $(\phi=0.8)$, stoichiometric $(\phi=1)$ and rich $(\phi=1.2)$ methane-air explosion in a single cylinder with rear ignition and small $\left(67.9 \mathrm{~cm}^{2}\right)$ vent area.

For the post-processing of explosion videos in a single cylinder with rear ignition, a Matlab code was written to determine the flame tip position, velocity, burned gas volume and flame surface area versus time. Figure 24 depicts the flame tip position, Fig. 24a, and velocity, Fig. 24b, versus time for $\phi=0.8,1$ and 1.2 methane-air burning in a single cylinder with rear ignition and a small 
$\left(67.9 \mathrm{~cm}^{2}\right)$ vent area. It was seen that for all the compositions, flame accelerated in an exponential manner. In stoichiometric $\phi=1$ condition flame accelerated fastest which was followed by fuelrich $\phi=1.2$ and fuel-lean $\phi=0.8$ methane-air mixtures, respectively.
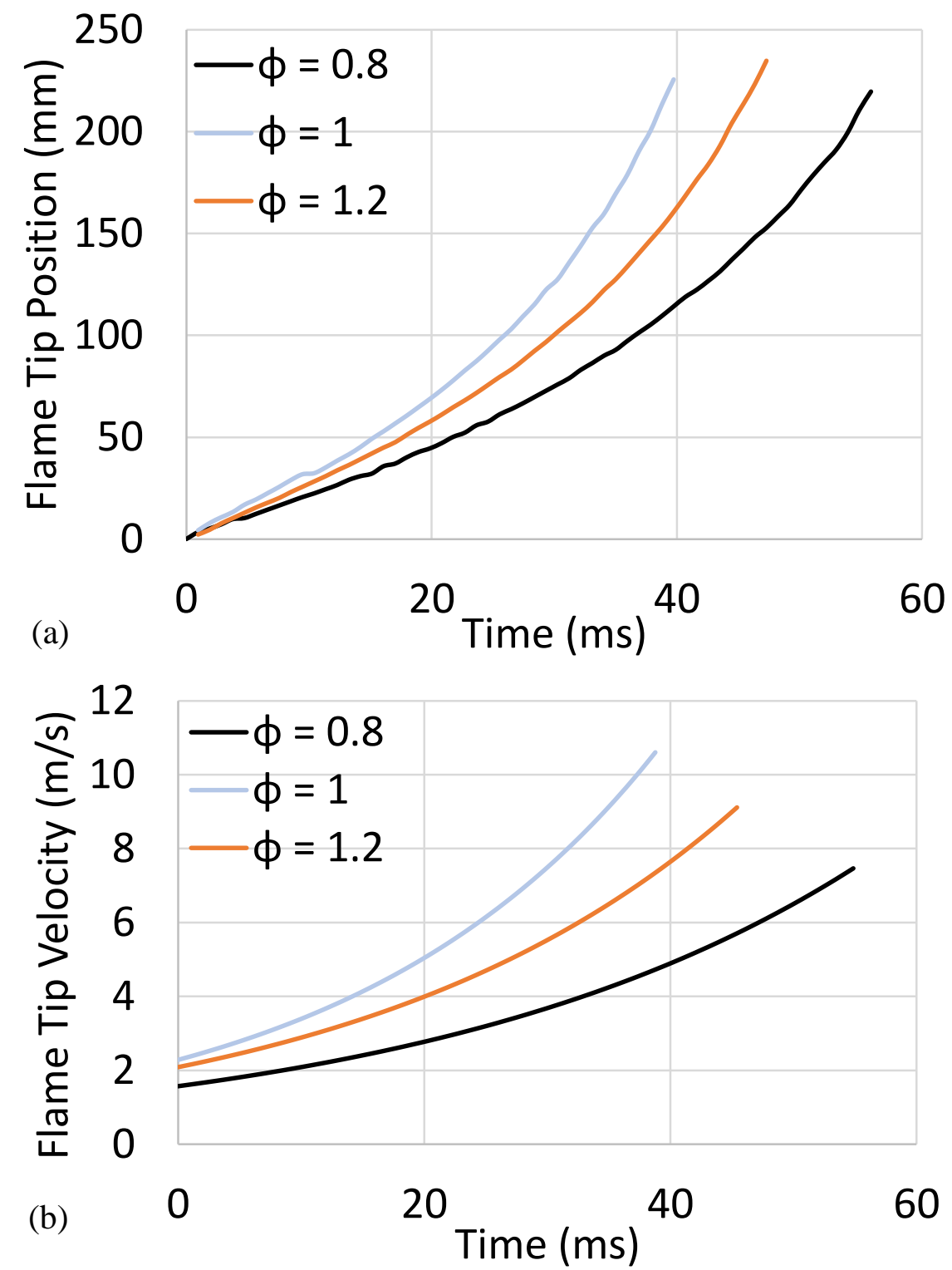

Figure 24: Time evolution of the flame tip position (a) and velocity (b) for lean $(\phi=0.8)$, stoichiometric $(\phi=1)$ and rich $(\phi=1.2)$ methane-air burnings in a single cylinder with rear ignition and a small vent area $\left(67.9 \mathrm{~cm}^{2}\right)$.

Figure 25 presents the flame surface area, Fig. 25a, and the volume of the burnt gas, Fig. 25b, versus time for the $\phi=0.8,1$ and 1.2 methane-air explosions in a single cylinder with rear ignition and a small $\left(67.9 \mathrm{~cm}^{2}\right)$ vent size. It is seen that both the burned gas volume and the flame surface area grew exponentially. As the flame elongated towards the vent and acquired a finger-like shape, 
the flame surface area increased resulting in a faster increase in the burned gas volume. Fastest growth of both quantities was seen in the stoichiometric case; followed by the rich and lean mixtures respectively.
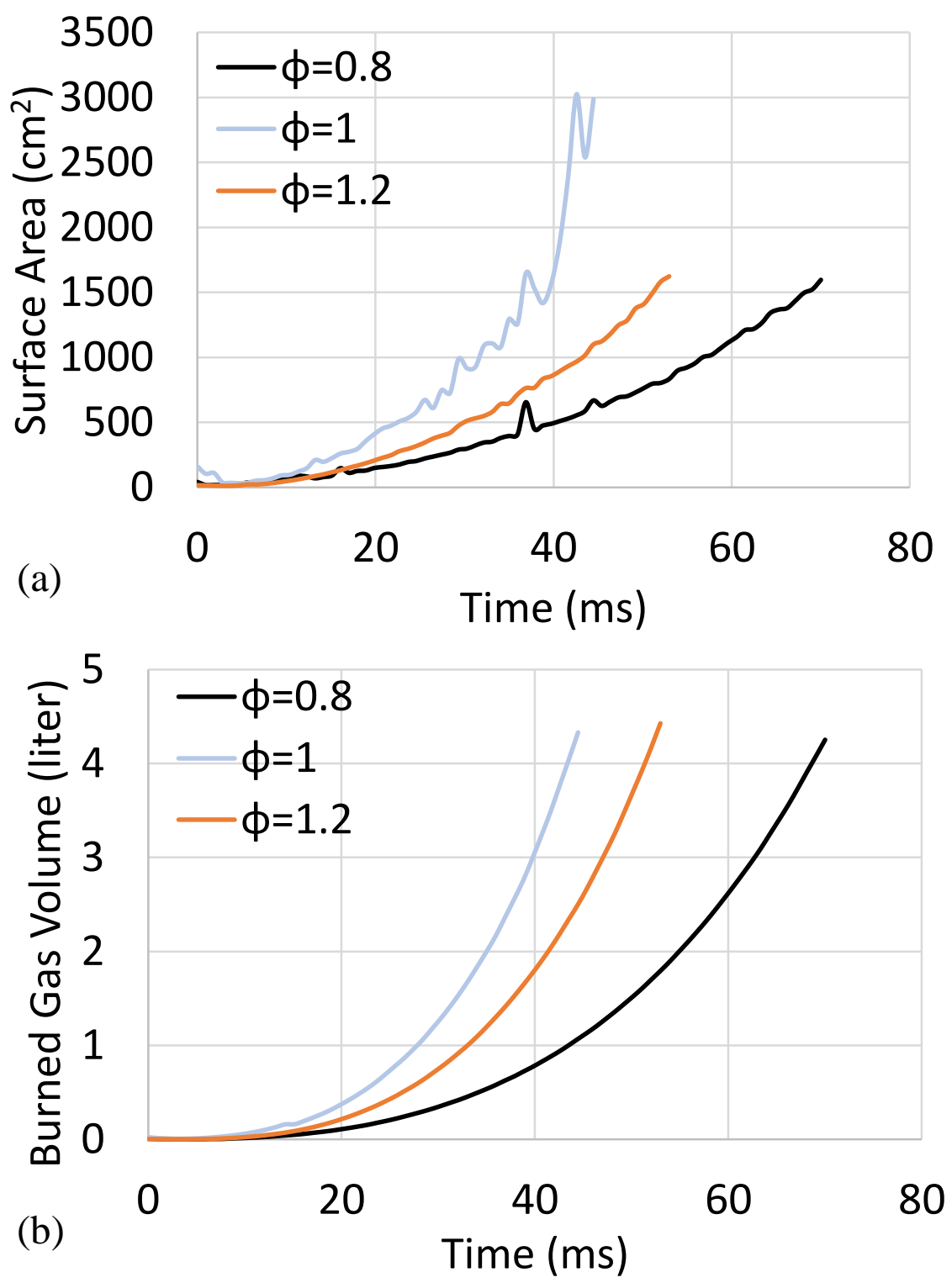

Figure 25: Evolutions of the flame surface area (a) and burned gas volume (b) for the lean $(\phi=0.8)$, stoichiometric $(\phi=1)$ and rich $(\phi=1.2)$ methane-air explosions in a single cylinder with a small (67.9 $\mathrm{cm}^{2}$ ) vent area and rear ignition.

Figure 26 presents the evolution of the pressure, Fig. 26a, and that of the rate of pressure rise, Fig. $266 \mathrm{~b}$, for the $\phi=0.8,1$ and 1.2 methane-air explosions in a single cylinder with rear ignition and a medium $\left(86.6 \mathrm{~cm}^{2}\right)$ vent. It is seen that the highest maximum pressure, $P_{\max }=0.017 \mathrm{bar}-\mathrm{g}$, was obtained in the stoichiometric case; followed by $P_{\max }=0.016$ and 0.013 bar-g for $\phi=1.2$ and 
0.8, respectively. Again, stoichiometric conditions provided the highest maximum rate of pressure rise, $(d P / d t)_{\max }=1.547 \mathrm{bar}-\mathrm{g} / \mathrm{s}$; followed by $(d P / d t)_{\max }=1.516$ and $1.202 \mathrm{bar}-\mathrm{g} / \mathrm{s}$, for the rich and lean conditions, respectively. An intriguing feature of the pressure history was observed in the case of rich $(\phi=1.2)$ burning such that the shaded areas showing the error in three repeated experiments are very large. This is due to the fact that the different timings and thereby different histories of pressure and pressure peaks was obtained in this condition of experiments. However, the magnitudes of pressure peaks were similar in the three repeated experiments.

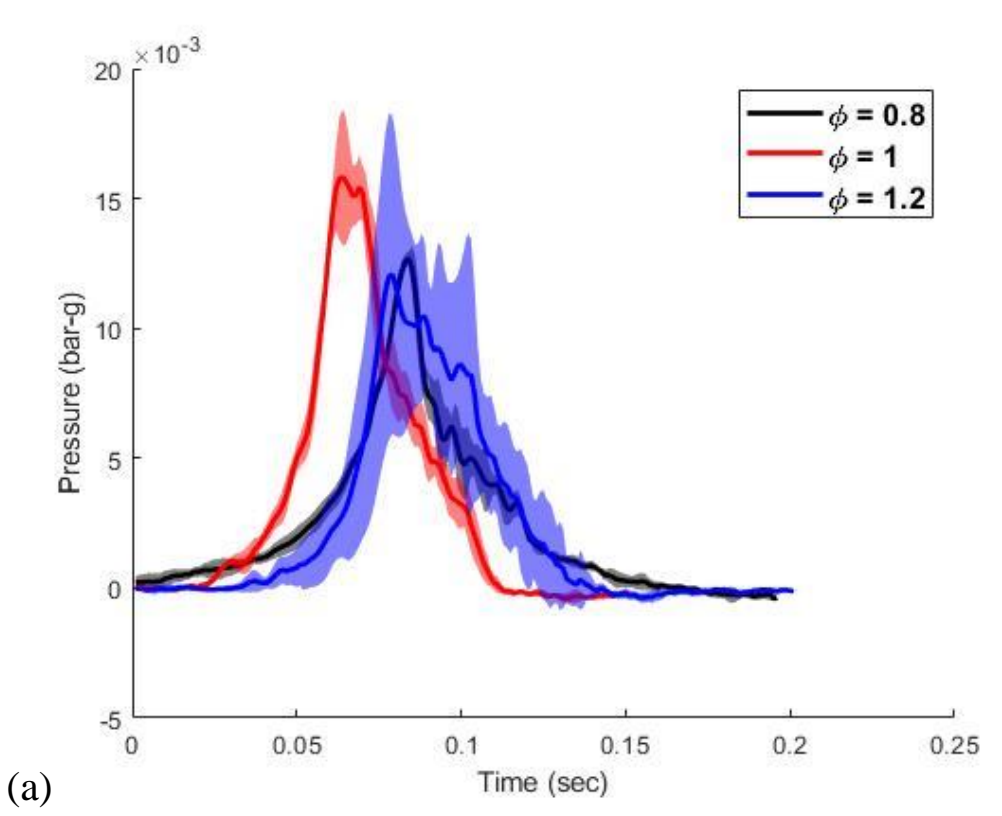

(a)

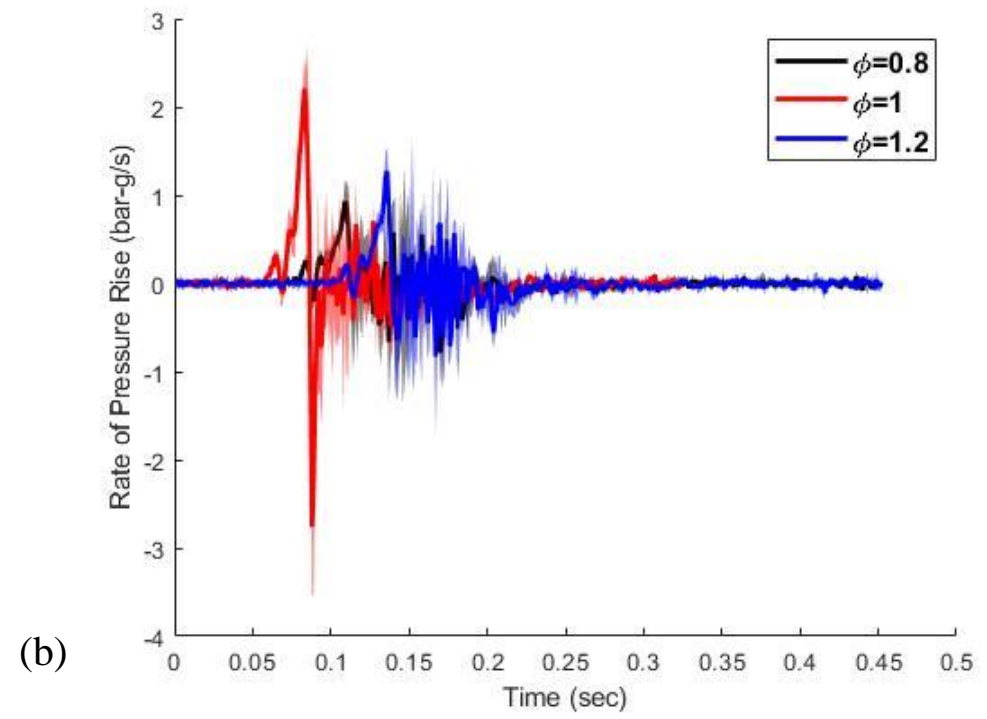

Figure 26: Time evolution of pressure (a) and that of the rate of pressure rise (b) for the lean $(\phi=0.8)$, stoichiometric $(\phi=1)$ and rich $(\phi=1.2)$ methane-air explosions in a single cylinder with rear ignition and a medium $\left(86.6 \mathrm{~cm}^{2}\right)$ vent area. 

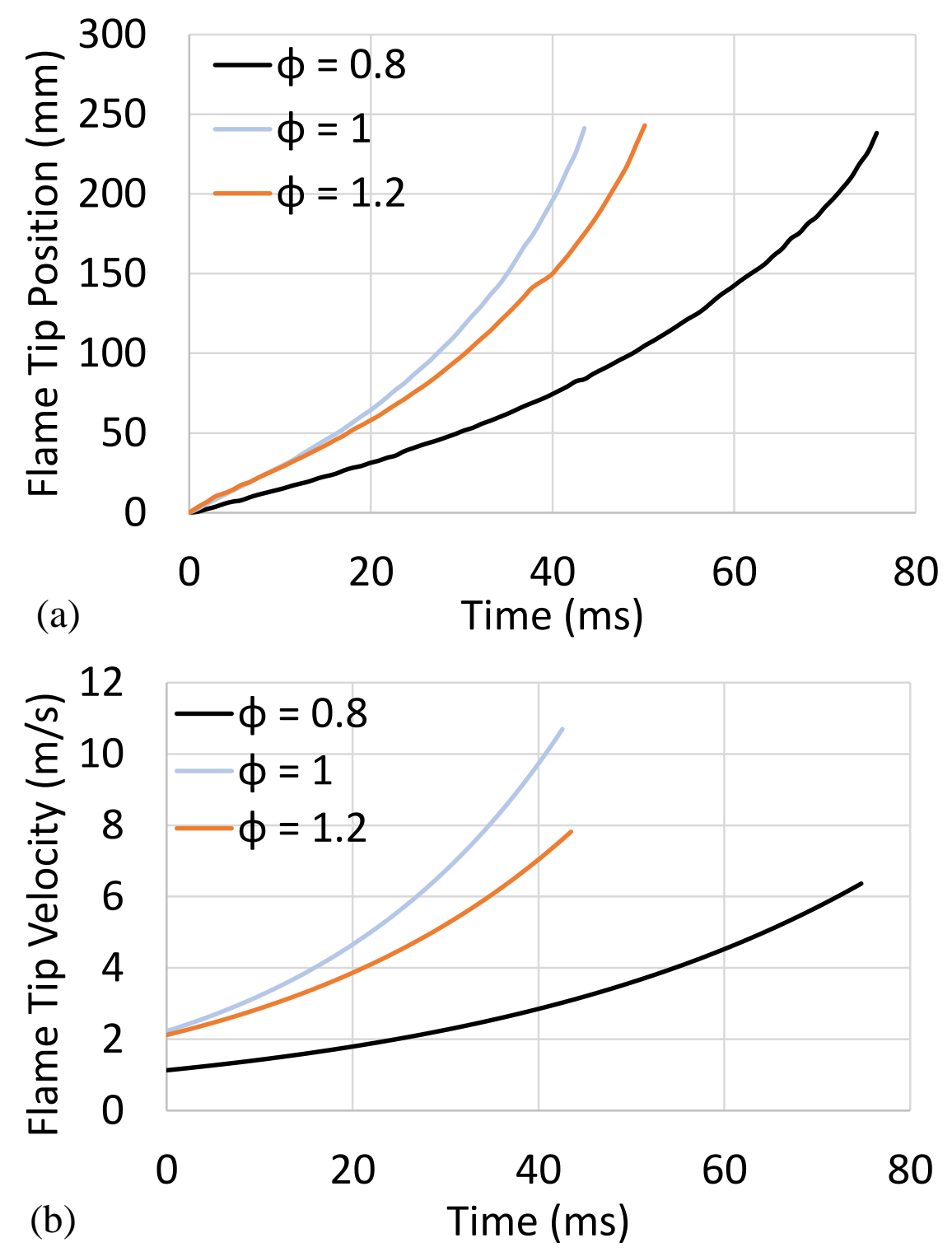

Figure 27: Evolutions of the flame tip position (a) and velocity (b) for the lean $(\phi=0.8)$, stoichiometric $(\phi=1)$ and rich $(\phi=1.2)$ methane-air explosions in a single cylinder with a medium $\left(86.6 \mathrm{~cm}^{2}\right)$ vent area and rear ignition.

Figure 27 depicts the evolutions of the flame tip position, Fig. 27a, and velocity, Fig. 27b, for the $\phi=0.8,1$ and 1.2 methane-air explosions in a single cylinder with a medium $\left(86.6 \mathrm{~cm}^{2}\right)$ vent size and rear ignition. In all three cases, the flame velocity grew exponentially. Strongest acceleration was observed in the stoichiometric case; followed by the rich and lean ones. Before venting occurred, the flame tip velocities attained the values $U_{\text {tip }}=6.4,10.7,9.3 \mathrm{~m} / \mathrm{s}$ for $\phi=0.8,1,1.2$, respectively. 

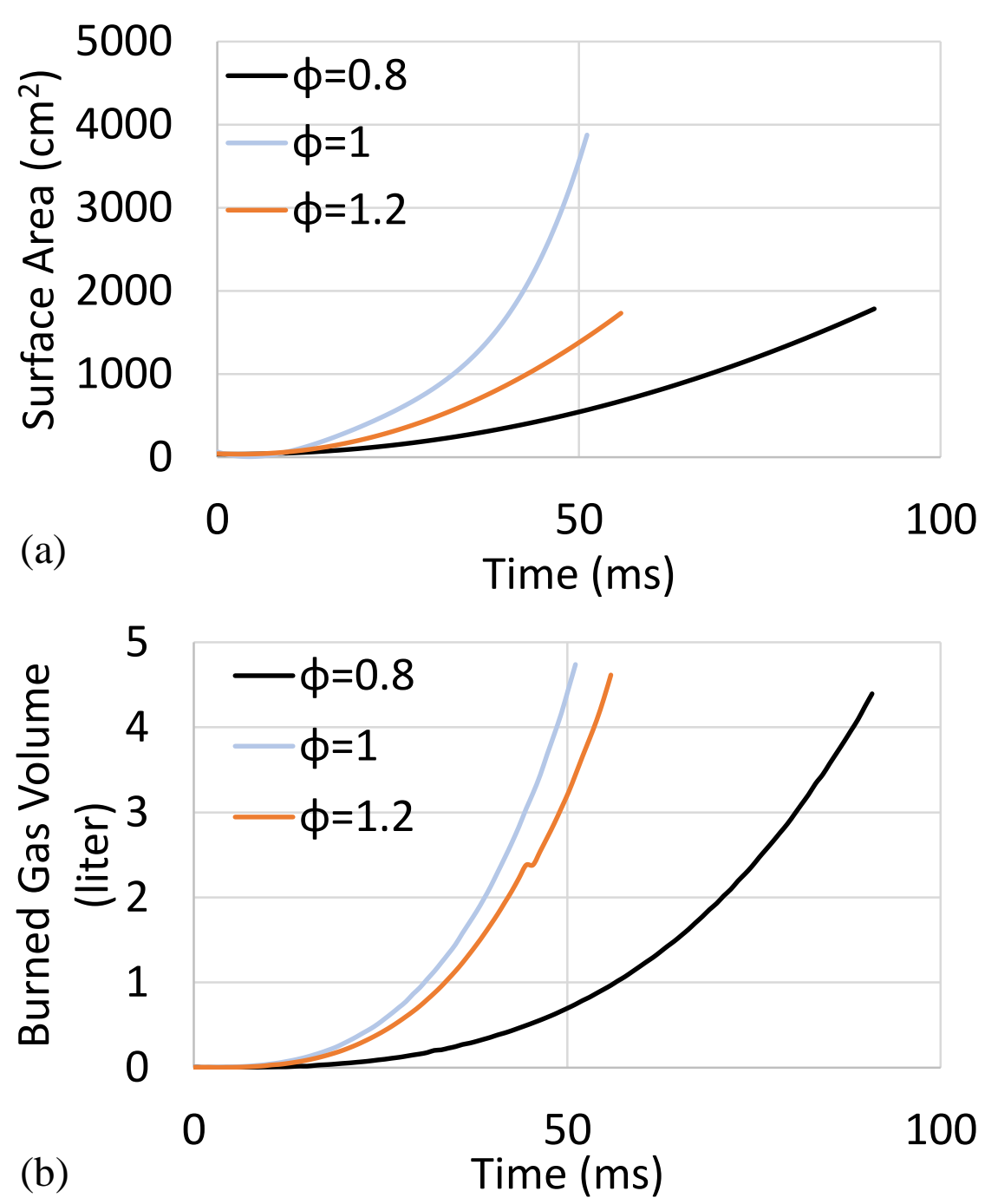

Figure 28: Evolutions of the flame surface area and the burned gas volume for fuel-lean $\phi=0.8$, stoichiometric $\phi=1$ and fuel-rich $\phi=1.2$ methane-air explosions in a single cylinder with medium $\left(86.6 \mathrm{~cm}^{2}\right)$ vent and rear ignition.

Figure 28 shows the evolutions of the flame surface area, Fig. 28a, and of the burnt gas volume, Fig. 28b, for the $\phi=0.8,1$ and 1.2 methane-air explosions in a single cylinder with a medium $\left(86.6 \mathrm{~cm}^{2}\right)$ vent and central ignition. For all three methane-air mixture compositions considered, the flame surface area grew rapidly in an exponential manner, burning more fresh fuel per unit time and thereby resulting in an exponential increase in the burned gas volume.

Figure 29 presents the evolution of pressure, Fig. 29a, and that of the rate of pressure rise, Fig. $29 \mathrm{~b}$, for the $\phi=0.8,1$ and 1.2 explosions in a single cylinder with a large $\left(132.7 \mathrm{~cm}^{2}\right)$ vent size and rear ignition. As expected, the highest maximum pressure, $P_{\max }=0.017 \mathrm{bar}-\mathrm{g}$, corresponded to the stoichiometric conditions; followed by $P_{\max }=0.012$ and 0.007 bar-g for $\phi=0.8$ and $\phi=$ 
1.2, respectively. Similarly, according to Fig. 29b, stoichiometric burning provided the highest maximum rate of pressure rise, $(d P / d t)_{\max }=1.952 \mathrm{bar}-\mathrm{g} / \mathrm{s}$; followed by $1.327 \mathrm{bar}-\mathrm{g} / \mathrm{s}$ and $0.478 \mathrm{bar}-\mathrm{g} / \mathrm{s}$ for the lean and rich mixtures, respectively.
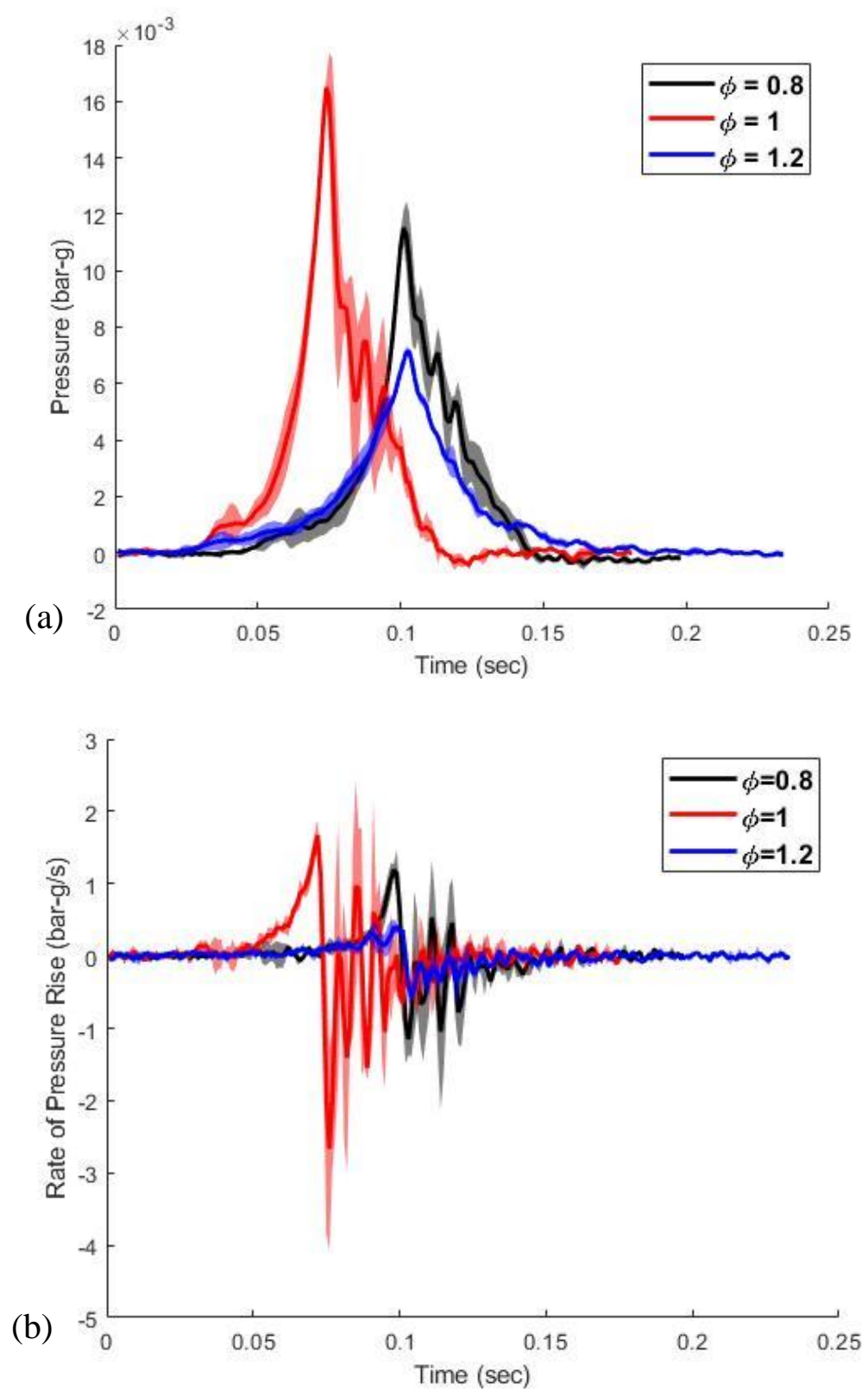

Figure 29: Time evolution of pressure (a) and that of the rate of pressure rise (b) for the lean $(\phi=0.8)$, stoichiometric $(\phi=1)$ and rich $(\phi=1.2)$ methane-air explosions in a single cylinder with a large vent area $\left(132.7 \mathrm{~cm}^{2}\right)$ and rear ignition.

Figure 30 presents the evolution of the flame tip position, Fig. 30a, and velocity, Fig. 30b, for the $\phi=0.8,1$ and 1.2 explosions in a single cylinder with a large $\left(132.7 \mathrm{~cm}^{2}\right)$ vent and rear ignition. It is seen that flames accelerated exponentially in all these cases, with fastest acceleration observed 
in the stoichiometric case; followed by lean $(\phi=0.8)$ and rich $(\phi=1.2)$ burning, respectively. Before venting occurred, the flame tip velocities were $9,9.9,7.3 \mathrm{~m} / \mathrm{s}$ for $\phi=0.8,1,1.2$, respectively.
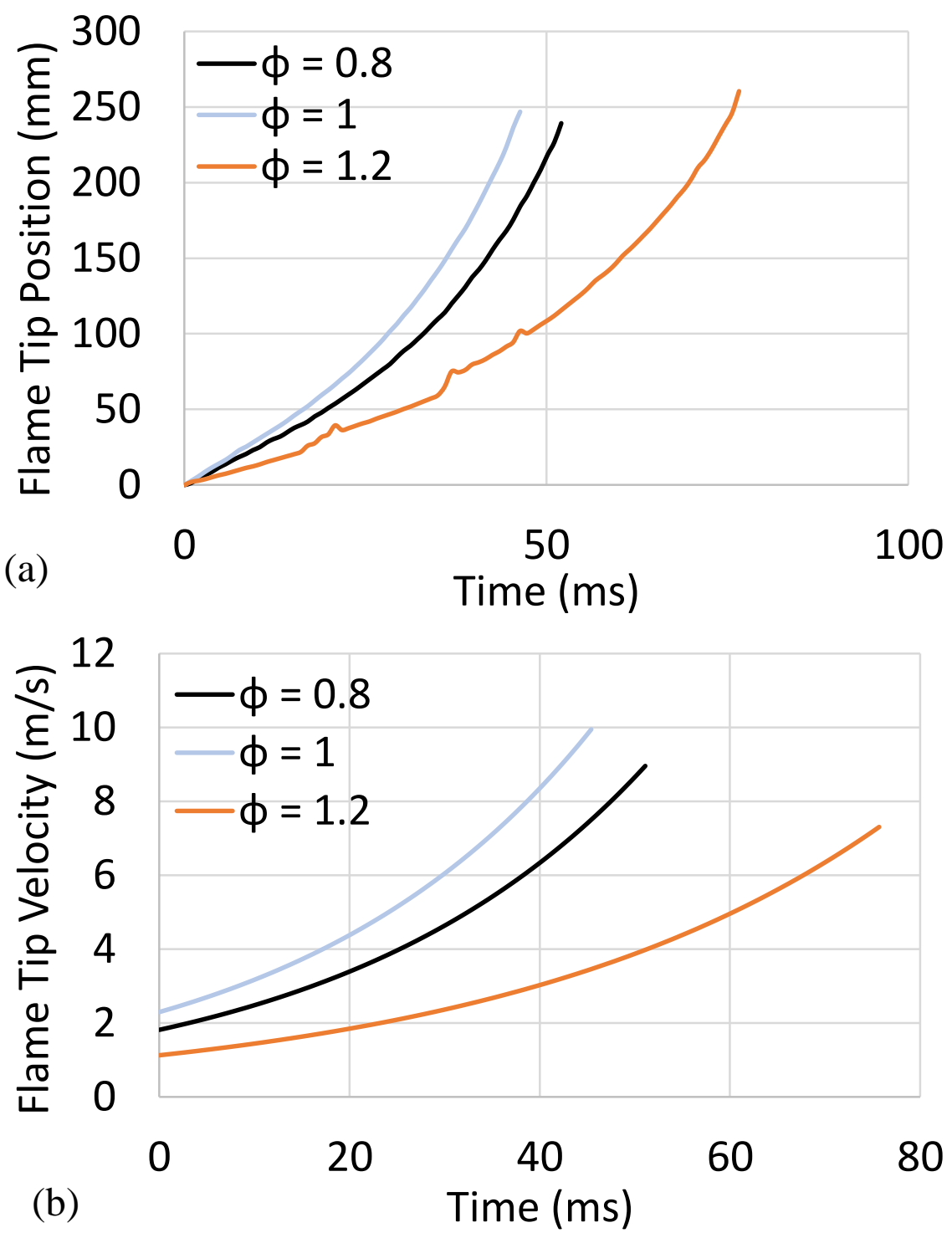

Figure 30: Evolutions of the flame tip position (a) and velocity (b) of fuel-lean $\phi=0.8$, stoichiometric $\phi=1$ and fuel-rich $\phi=1.2$ methane-air explosions in a single cylinder with large $\left(132.7 \mathrm{~cm}^{2}\right)$ vent and rear ignition.

Figure 31 presents the evolutions of the flame surface area, Fig. 31a, and of the burned gas volume, Fig. 31b, for the $\phi=0.8,1$ and 1.2 methane-air explosions in a single cylinder with a large (132.7 $\mathrm{cm}^{2}$ ) vent area and rear ignition. It is seen that the flame surface area grew exponentially, thereby resulting in an exponential increase in the burned gas volume as shown in Fig. 31b. 

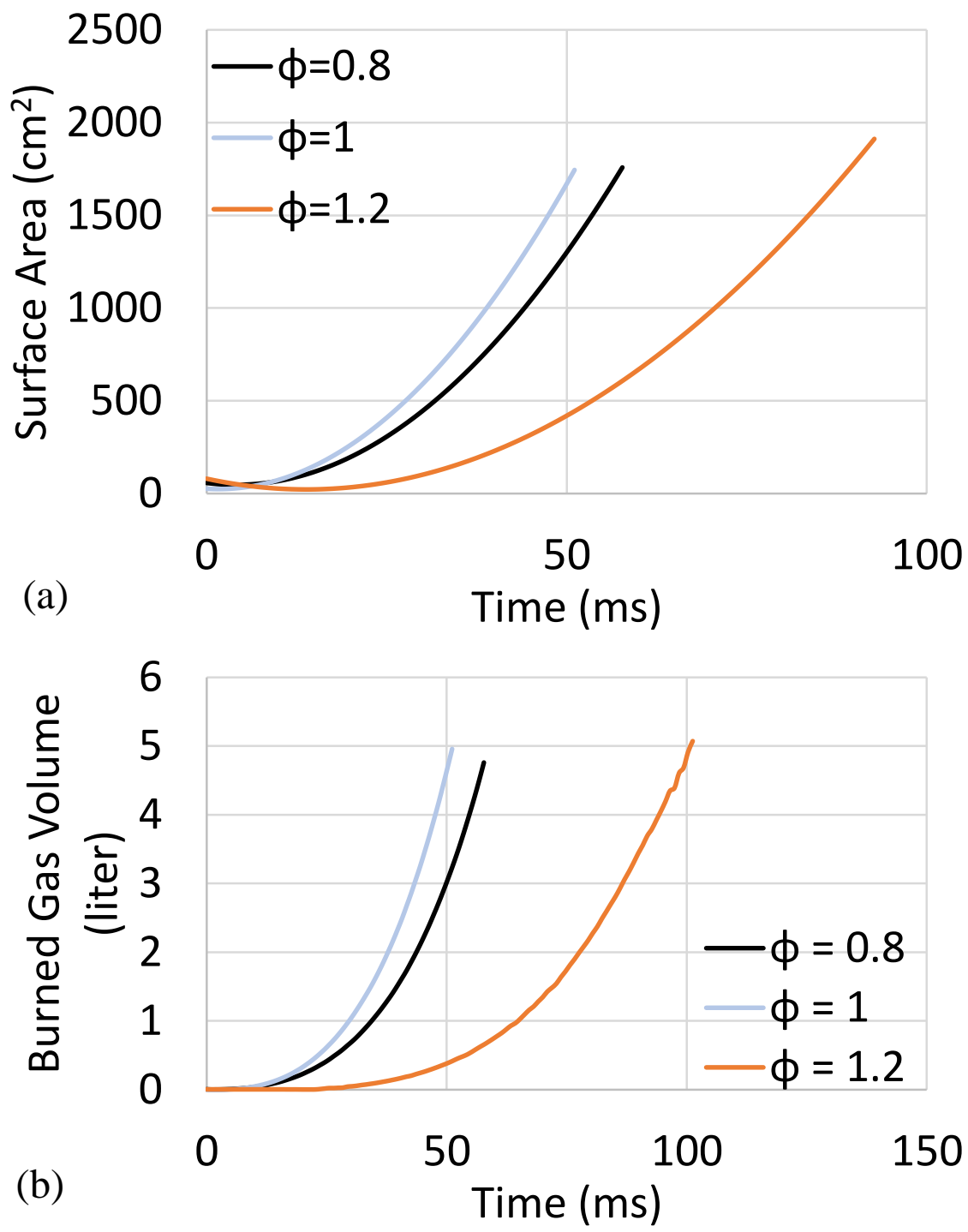

Figure 31: Evolutions of the flame surface area (a) and of the burnt gas volume (b) for the lean $(\phi=0.8)$, stoichiometric $(\phi=1)$ and rich $(\phi=1.2)$ methane-air explosions in a single cylinder with a large (132.7 $\mathrm{cm}^{2}$ ) vent and rear ignition.

Figure 32 depicts the acceleration rate $\sigma$ versus the equivalence ratio $\phi$ of a methane-air mixture burning in a single cylinder with rear ignition for all three vent areas. It is seen that, in the case of stoichiometric and rich $(\phi=1.2)$ flames, as the vent size diminished from large $\left(132.7 \mathrm{~cm}^{2}\right)$ to small $\left(67.9 \mathrm{~cm}^{2}\right)$, the acceleration rate increased. The only discrepancy was in lean $(\phi=0.8)$ burning with a large $\left(132.7 \mathrm{~cm}^{2}\right)$ vent size that resulted in the highest acceleration rate. 


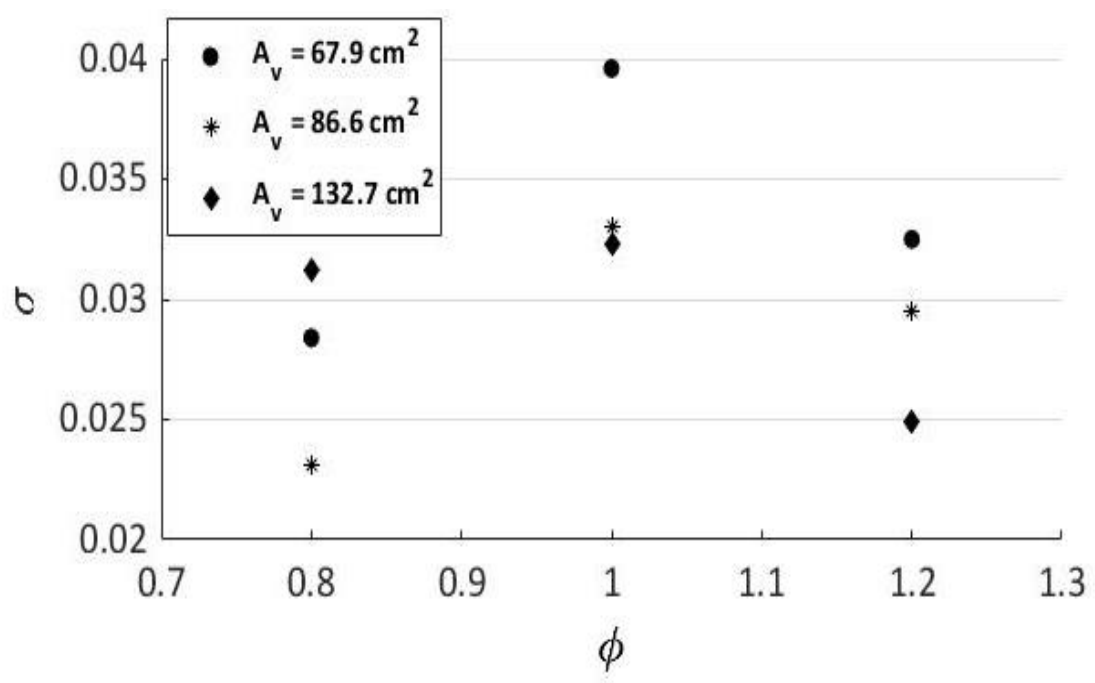

Figure 32: The exponential acceleration factor $\sigma$ versus the equivalence ratio $\phi$ for methane-air explosions in a single cylinder with rear ignition.

\subsubsection{Extended Single Cylinder}

Schematic of the experimental setup is shown in Fig. 12, with only one modification: the length of the cylinder increased from $30 \mathrm{~cm}$ to $60 \mathrm{~cm}$. Figure 33 shows the evolution of pressure (a), and that of the rate of pressure rise (b), for the $\phi=0.8,1$ and 1.2 methane-air explosions with a small $\left(67.9 \mathrm{~cm}^{2}\right)$ vent size and rear ignition in an extended single cylinder. As expected, the highest maximum pressure as $P_{\max }=0.099$ bar-g was observed in the stoichiometric case; followed by 0.086 and 0.042 bar-g for $\phi=1.2$ and 0.8 , respectively. Stoichiometric burning also provided the highest maximum rate of pressure rise, $(d P / d t)_{\max }=5.69 \mathrm{bar}-\mathrm{g} / \mathrm{s}$, which was followed by 4.81 and $3.143 \mathrm{bar}-\mathrm{g} / \mathrm{s}$, for $\phi=1.2$ and $\phi=0.8$, respectively.

Figure 34 presents the evolutions of the flame tip position, Fig. 34a, and velocity, Fig. 34b, for the $\phi=0.8,1$ and 1.2 methane-air explosions in an extended (60 $\mathrm{cm}$ length) single cylinder with a small $\left(67.9 \mathrm{~cm}^{2}\right)$ vent size and rear ignition. It is seen that for all methane-air mixture compositions, the flame tip accelerated, and this acceleration was exponential. Fastest acceleration was observed for stoichiometric combustion; followed by the rich and lean cases, respectively. Before the venting occurred, the flame tip velocity reached the values of $U_{t i p}=14.7 \mathrm{~m} / \mathrm{s}$ for $\phi=0.8 ; U_{\text {tip }}=$ $22.8 \mathrm{~m} / \mathrm{s}$ for the stoichiometric case; and $U_{t i p}=21.1 \mathrm{~m} / \mathrm{s}$ for $\phi=1.2$. 

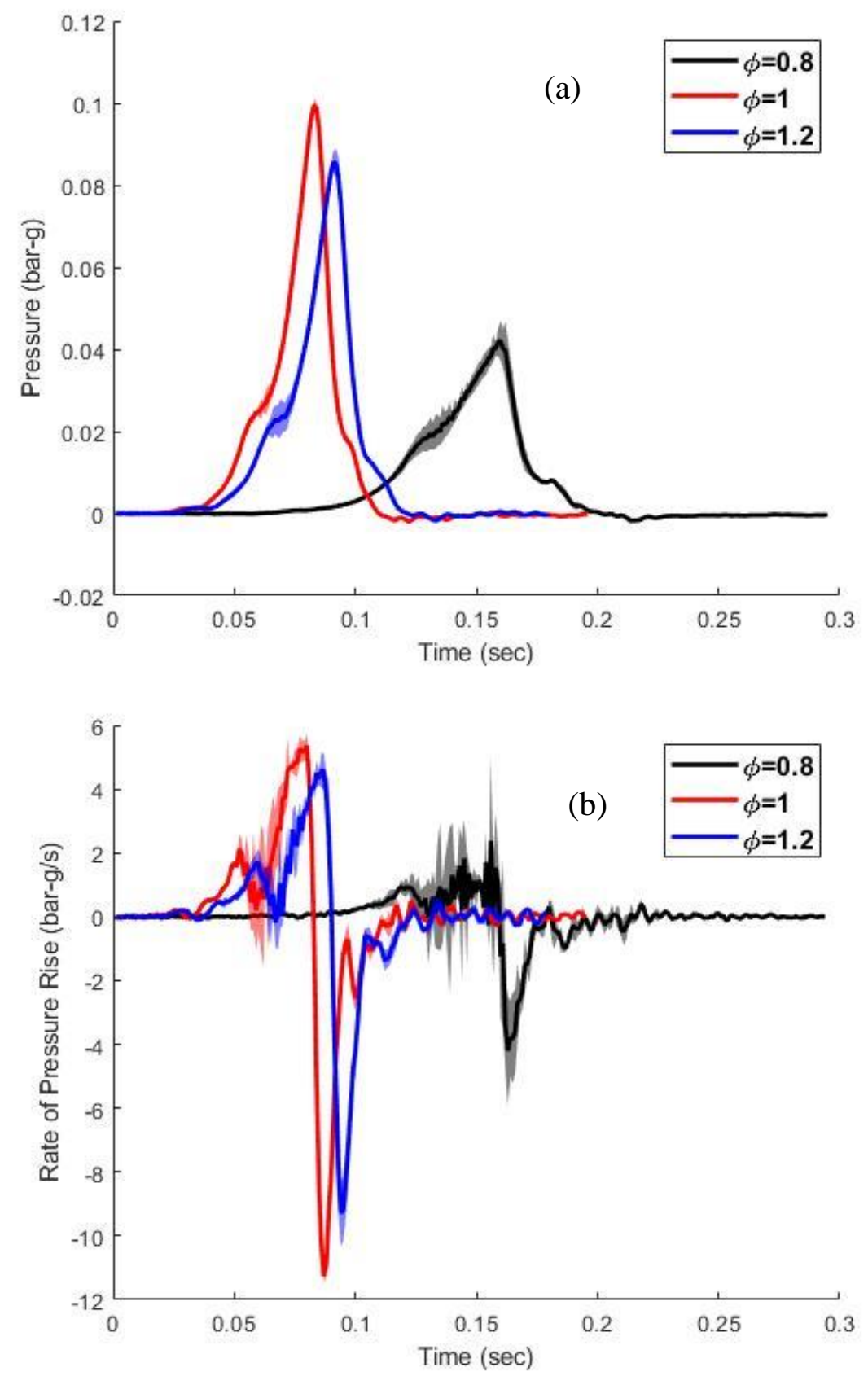

Figure 33: Time evolution of pressure (a) and that of the rate of pressure rise (b) for the lean $(\phi=0.8)$, stoichiometric $(\phi=1)$ and rich $(\phi=1.2)$ methane-air explosions in the extended $(60 \mathrm{~cm} \mathrm{long})$ single cylinder with a small $\left(67.9 \mathrm{~cm}^{2}\right)$ vent area and rear ignition. 

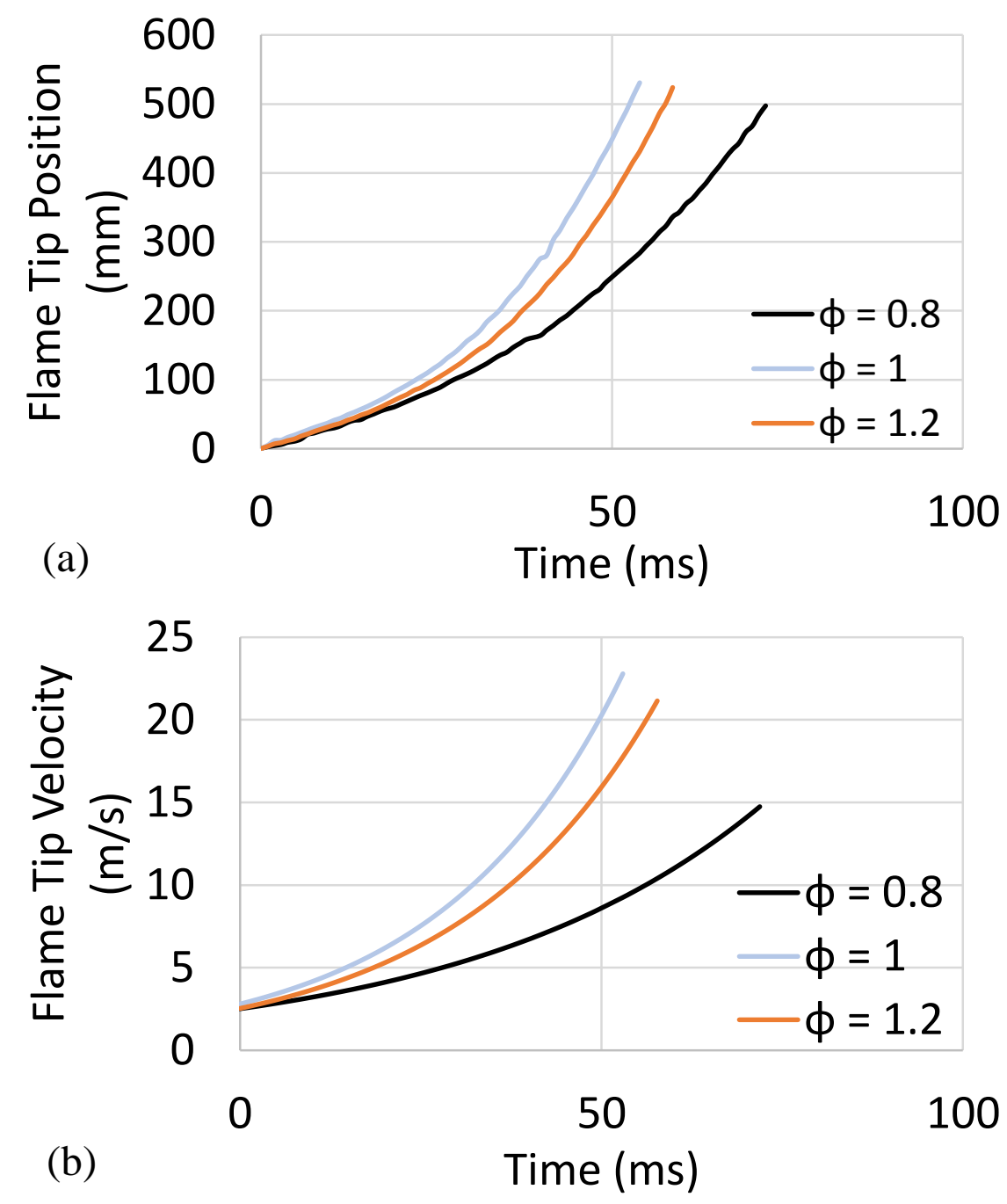

Figure 34: Time evolution of flame tip position (a) and velocity (b) for the lean $(\phi=0.8)$, stoichiometric $(\phi=1)$ and rich $(\phi=1.2)$ methane-air explosions in the extended $(60 \mathrm{~cm}$ long $)$ single cylinder with a small $\left(67.9 \mathrm{~cm}^{2}\right)$ vent area and rear ignition.

Figure 35 shows the evolution of pressure, Fig. 35a, and that of the rate of pressure rise, Fig. 35b, for the $\phi=0.8,1$ and 1.2 methane-air explosions in an extended single cylinder of length $60 \mathrm{~cm}$ with a medium $\left(86.6 \mathrm{~cm}^{2}\right)$ vent size and rear ignition. It is seen that the highest maximum pressure, $P_{\max }=0.069$ bar-g, was obtained at the stoichiometric conditions, followed by $P_{\max }=0.063$ bar-g for $\phi=1.2$ and $P_{\max }=0.026$ bar-g for $\phi=0.8$. As for the pressure rate, again, the highest one, $5.074 \mathrm{bar}-\mathrm{g} / \mathrm{s}$, was when $\phi=1$; followed by $4.772 \mathrm{bar}-\mathrm{g} / \mathrm{s}$ and $1.360 \mathrm{bar}-\mathrm{g} / \mathrm{s}$ for $\phi=1.2$ and $\phi=0.8$, respectively. 

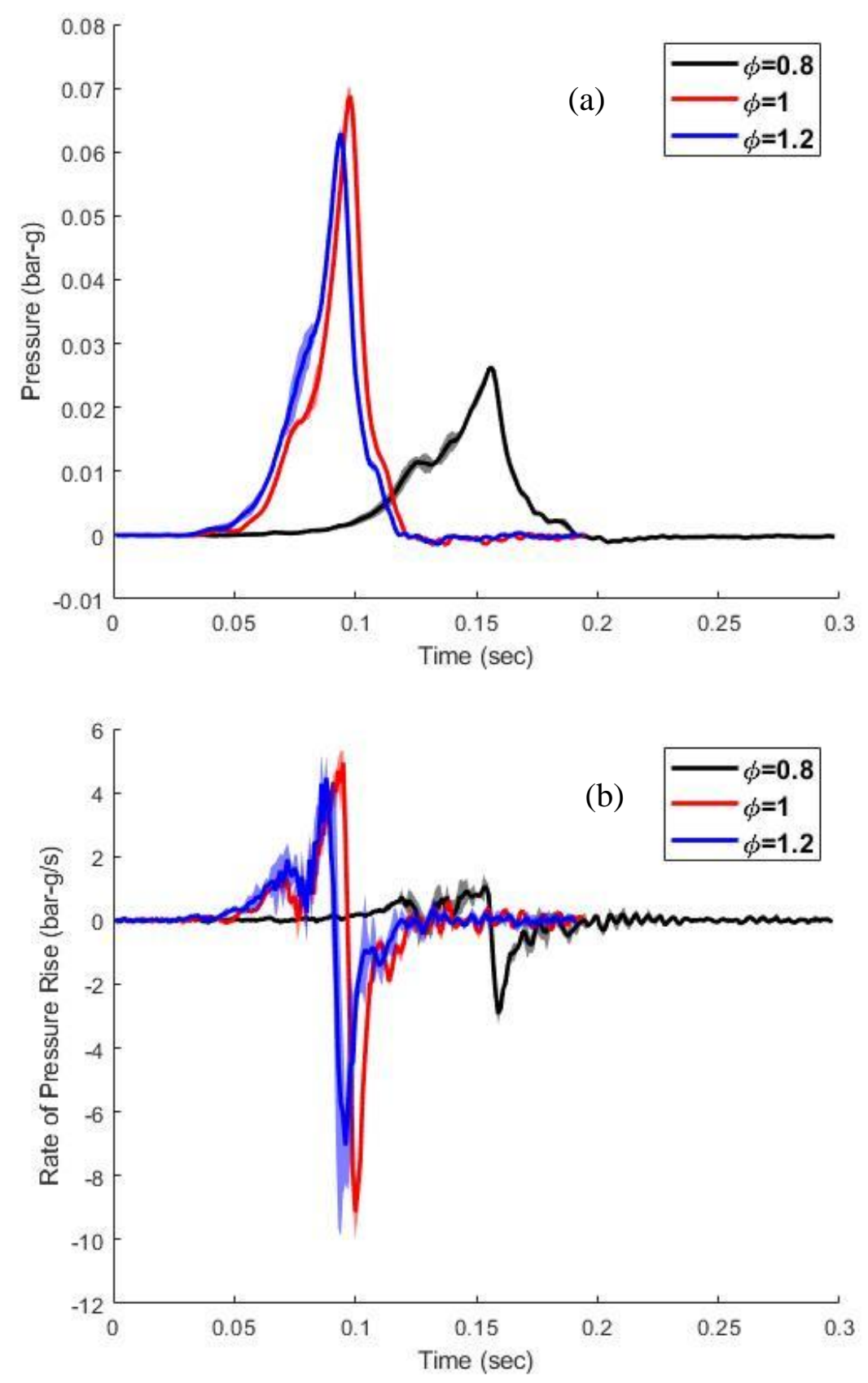

Figure 35: Time evolution of pressure (a) and of the rate of pressure rise (b) for the lean $(\phi=0.8)$, stoichiometric $(\phi=1)$ and rich $(\phi=1.2)$ methane-air explosions in the extended $(60 \mathrm{~cm} \mathrm{long})$ single cylinder with a medium $\left(86.6 \mathrm{~cm}^{2}\right)$ vent area and rear ignition.

Figure 36 shows the evolution of the flame tip position, Fig. 36a, and velocity, Fig. 36b, for the $\phi=0.8,1$ and 1.2 methane-air explosions in the extended $(60 \mathrm{~cm})$ single cylinder with a medium $\left(86.6 \mathrm{~cm}^{2}\right)$ vent area and rear ignition. For all the mixture compositions considered, the flame tip accelerated exponentially, with fastest acceleration observed in the stoichiometric case followed 
by the rich ( $\phi=1.2$ ) and lean ( $\phi=0.8)$ methane-air explosions, respectively. Before venting, the flame tip velocities were $U_{\text {tip }}=13.9 \mathrm{~m} / \mathrm{s}$ for $\phi=0.8 ; 24.4 \mathrm{~m} / \mathrm{s}$ for $\phi=1$; and $22 \mathrm{~m} / \mathrm{s}$ for $\phi=$ 1.2.

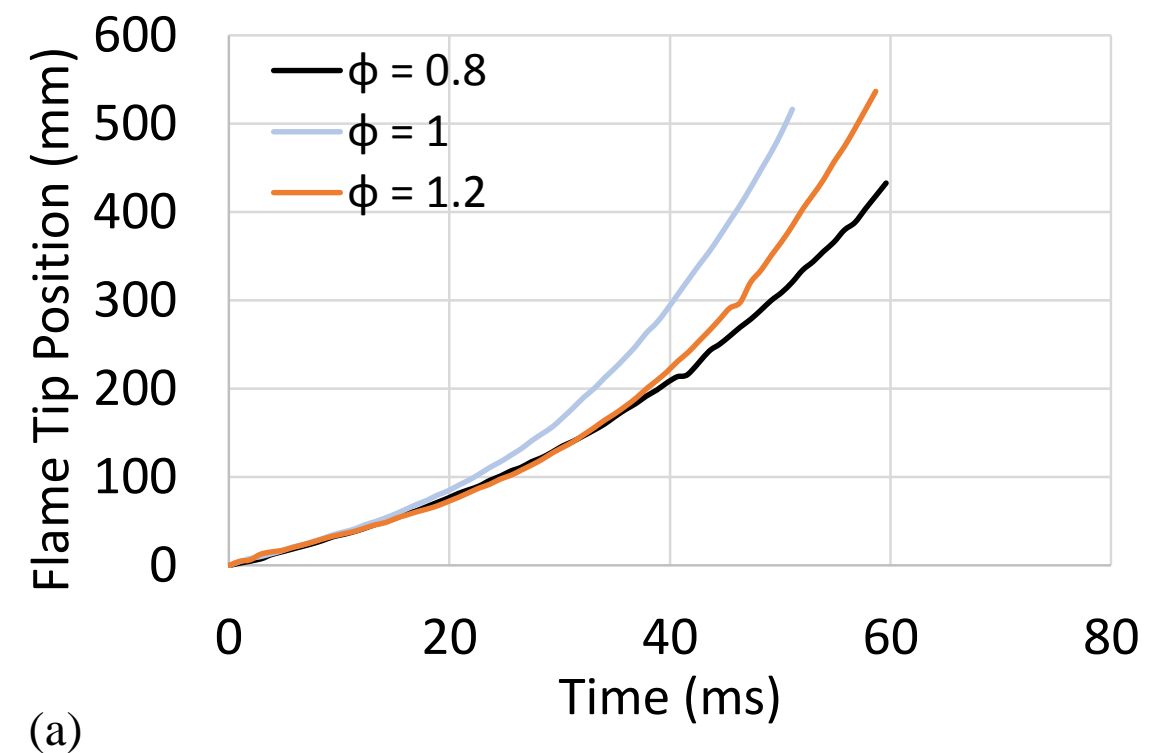

(a)

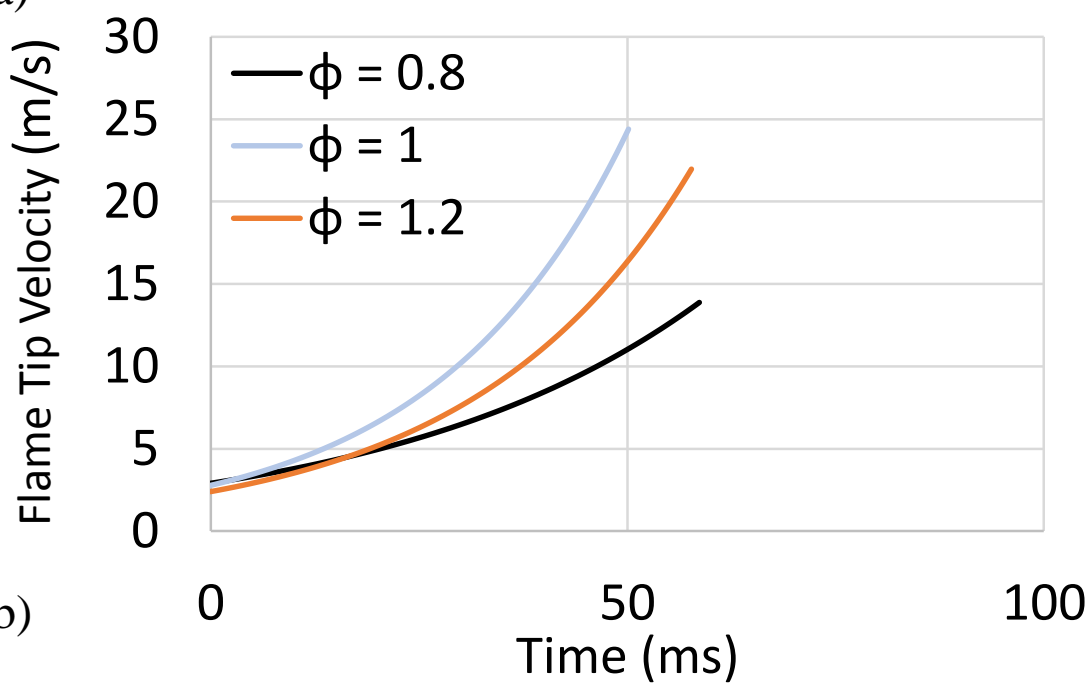

Figure 36: Evolutions of the flame tip position (a) and velocity (b) for the lean $(\phi=0.8)$, stoichiometric $(\phi=1)$ and rich $(\phi=1.2)$ methane-air explosions in the extended $(60 \mathrm{~cm} \mathrm{long})$ single cylinder with medium $\left(86.6 \mathrm{~cm}^{2}\right)$ vent and rear ignition.

Figure 37 shows the evolution of pressure, Fig. 37a, and that of the rate of pressure rise, Fig. 37b, for the $\phi=0.8$ and 1 explosions in the extended single cylinder $(60 \mathrm{~cm}$ long $)$ with large vent area $\left(132.7 \mathrm{~cm}^{2}\right)$ vent area and rear ignition. In this vent size of extended cylinder, the results of fuelrich $\phi=1.2$ methane-air explosions are not presented, because there was a suspicion regarding a measurement issue in the gas analyzer for this particular set of fuel-rich experiments. Figure 37 
demonstrates that the stoichiometric and lean cases resulted in the maximum pressures of $P_{\max }=$ 0.027 and 0.015 bar-g, respectively. The highest maximum rate of pressure rise obtained in the stoichiometric condition was much higher than that obtained in the fuel-lean methane-air condition.
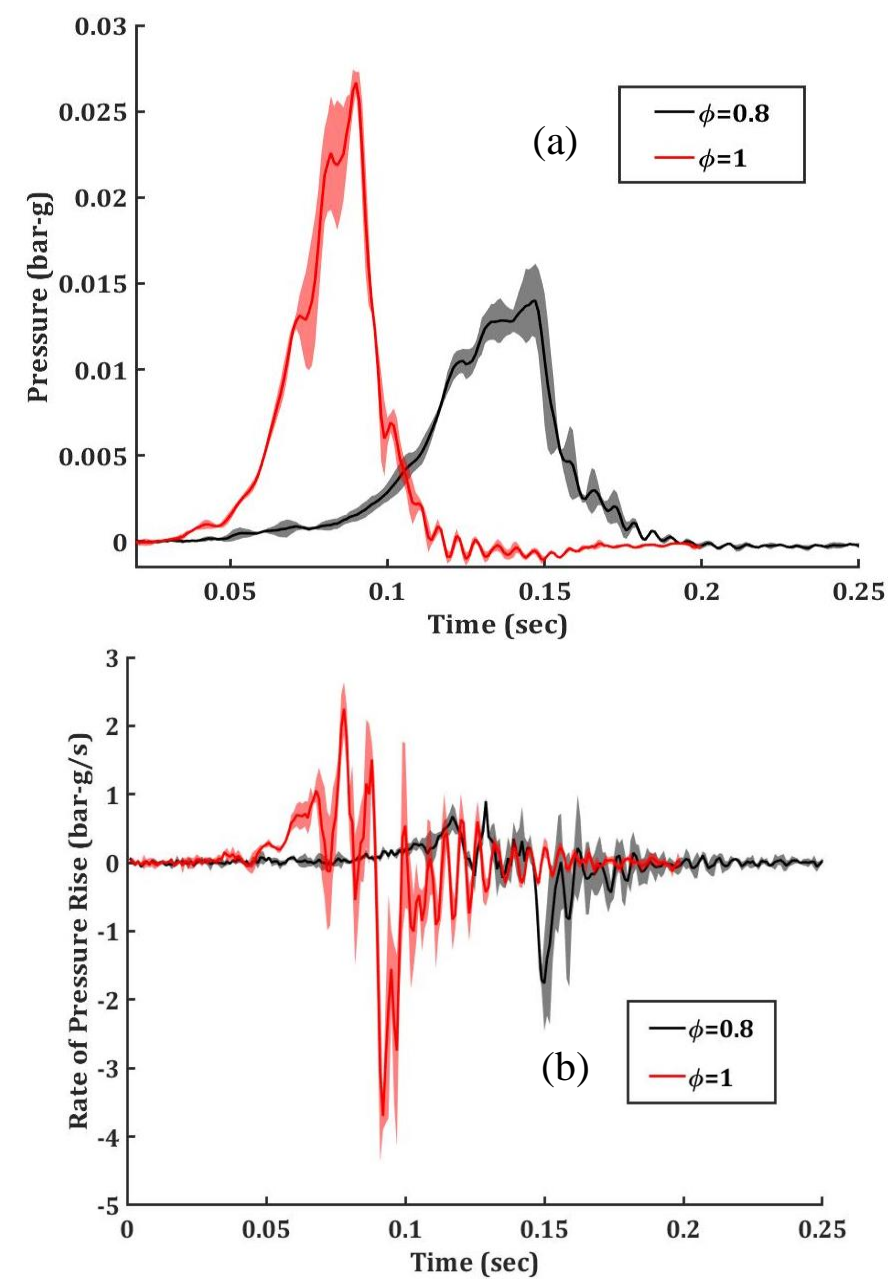

Figure 37: The evolution of pressure (a) and that of the rate of pressure raise (b) for the lean $(\phi=0.8)$ and stoichiometric $(\phi=1)$ methane-air explosions in an extended $(60 \mathrm{~cm}$ long) single cylinder with a large $\left(132.7 \mathrm{~cm}^{2}\right)$ vent area and rear ignition.

Figure 38 presents the evolution of the flame tip position, Fig. 38a, and velocity, Fig. 38b, for the $\phi=0.8$ and 1 explosions in an extended $(60 \mathrm{~cm})$ single cylinder with a large $\left(132.7 \mathrm{~cm}^{2}\right)$ vent and rear ignition. It is seen that for these mixture compositions, the flame tip accelerated in an exponential manner, with faster flame acceleration observed in the case of stoichiometric case. Before venting occurred, the flame tip velocities reached the values $U_{\text {tip }}=15$ and $23.2 \mathrm{~m} / \mathrm{s}$ for $\phi=0.8$ and 1 , respectively. 

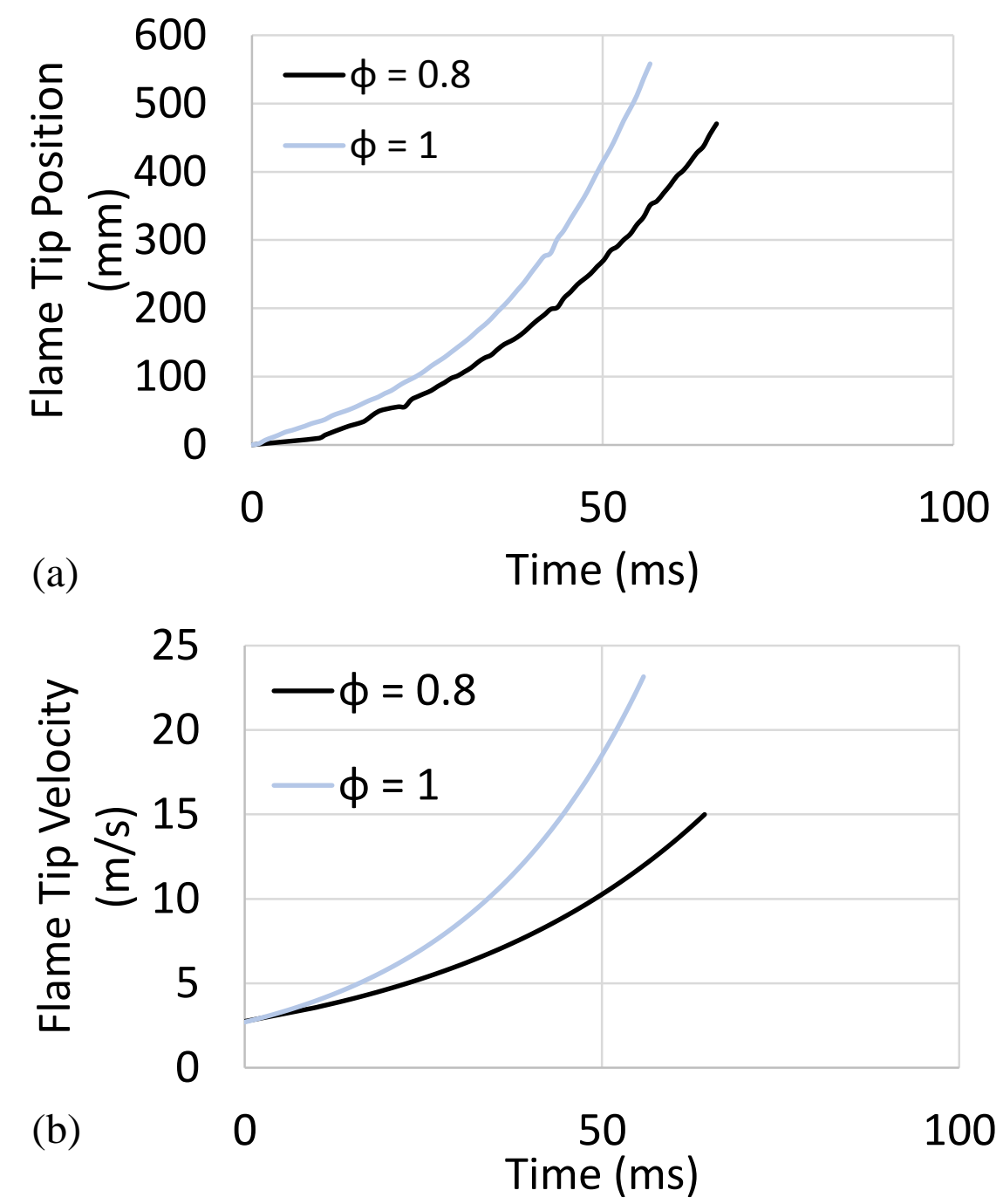

Figure 38: Evolutions of the flame tip position (a) and velocity (b) for the lean $(\phi=0.8)$ and stoichiometric $(\phi=1)$ methane-air explosions in an extended $(60 \mathrm{~cm}$ long) single cylinder with a large $\left(132.7 \mathrm{~cm}^{2}\right)$ vent area and rear ignition.

Figure 39 presents the exponential acceleration rate $\sigma$ versus the equivalence ratio $\phi$ for various vent areas for the extended single cylinder with rear ignition. It is seen that the relation between $\sigma$ and $\phi$ was not monotonic depending on the vent size. For the lean $(\phi=0.8)$ and stoichiometric methane-air explosions, the acceleration rate was the highest when the vent was medium (86.6 $\left.\mathrm{cm}^{2}\right)$, and it was the lowest when the vent was small $\left(67.9 \mathrm{~cm}^{2}\right)$. However, in the rich $(\phi=1.2)$ methane-air explosion, this behavior in acceleration rate was vice versa. 


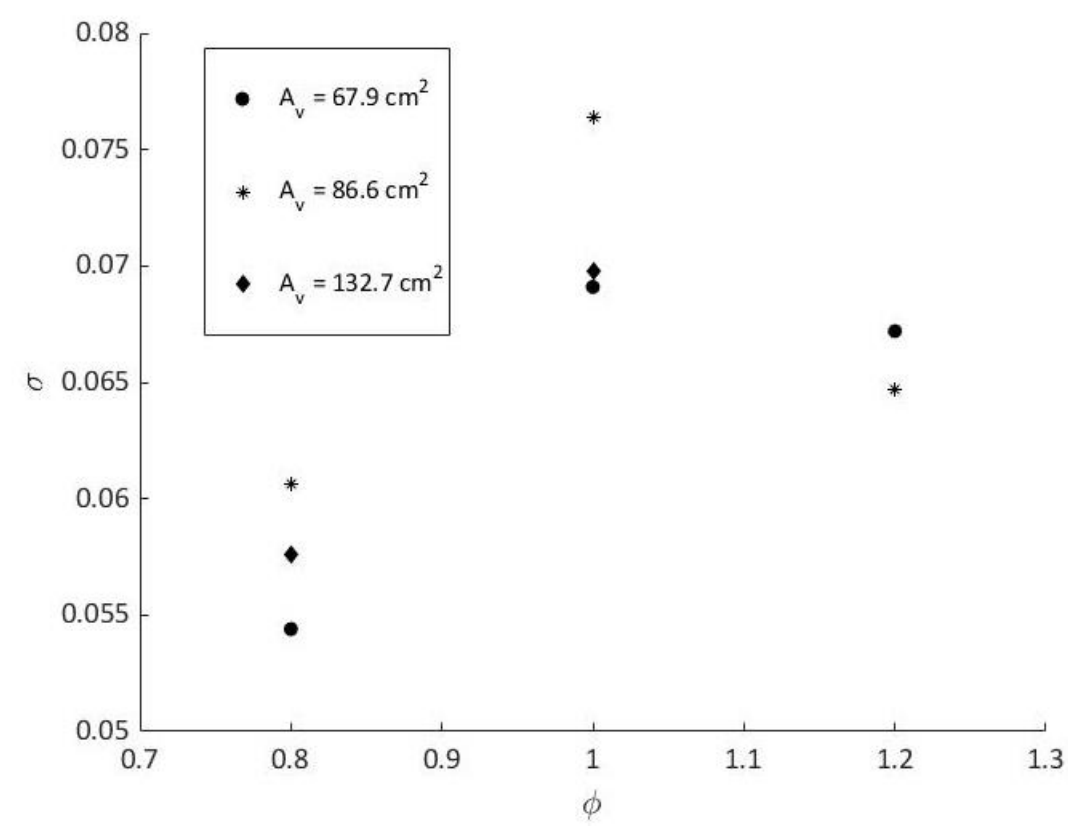

Figure 39: The exponential acceleration rate $\sigma$ versus the equivalence ratio $\phi$ for methane-air explosions in an extended (60 cm long) single cylinder with rear ignition.

Overall, the averaged maximum pressures $P_{\max , a v g}$ for each experimental configuration are listed in the Table 4, thereby showing that keeping all other experimental characteristics the same, the stoichiometric methane-air mixture resulted in the highest averaged maximum pressures in all set of experiments. Moreover, it is shown that for each mixture composition, increase in the vent area resulted in the decrease in the maximum average pressures $P_{\max , \text { avg }}$ for both the single (both rear and center ignition) and extended cylinders (rear ignition). Overall, the type of the enclosure seems to impact the maximum average pressure significantly in a way that as the length of the cylinder increased, the maximum average pressure results also increased.

Table 5 summarizes the exponential acceleration rates, $\sigma$, obtained from the experiments of single cylinder (rear and center ignition) and extended cylinder (rear ignition). In single cylinder experiments with center ignition, only the results of flame propagation towards vent direction are shown here because, flame propagating towards wall direction followed a constant velocity trend. In all set of experiments, the highest exponential acceleration rates were observed in the stoichiometric methane-air mixture. It is shown that in single cylinder experiments the exponential acceleration rates were higher when the mixture is centrally ignited. This is presumable due to the fact that in the center ignition case, the flame was initially closer to the vent, therefore venting effect occurred earlier, increasing the flame velocity towards the vent direction. Moreover, when 
the length of the cylinder was extended to twice longer (from $30 \mathrm{~cm}$ to $60 \mathrm{~cm}$ ), $\sigma$ also increased and this increase in $\sigma$ was more than double.

Table 4: Summary of maximum average pressures, $P_{\max , a v g}$.

\begin{tabular}{|c|c|c|c|}
\hline & \multicolumn{3}{|c|}{$P_{\text {max,avg }}($ bar-g) } \\
\hline & \multicolumn{3}{|c|}{ Small Vent $\left(67.9 \mathrm{~cm}^{2}\right)$} \\
\hline & \multicolumn{2}{|c|}{ Single Cylinder } & Extended Cylinder \\
\hline & Center Ignition & Rear Ignition & Rear Ignition \\
\hline Lean $(\phi=0.8)$ & 0.011 & 0.019 & 0.042 \\
\hline Stoichiometric $(\phi=1)$ & 0.019 & 0.032 & 0.099 \\
\hline \multirow[t]{4}{*}{$\operatorname{Rich}(\phi=1.2)$} & 0.013 & 0.025 & 0.086 \\
\hline & \multicolumn{3}{|c|}{ Medium Vent $\left(86.6 \mathrm{~cm}^{2}\right)$} \\
\hline & \multicolumn{2}{|c|}{ Single Cylinder } & Extended Cylinder \\
\hline & Center Ignition & Rear Ignition & Rear Ignition \\
\hline Lean $(\phi=0.8)$ & 0.01 & 0.013 & 0.026 \\
\hline Stoichiometric $(\phi=1)$ & 0.012 & 0.017 & 0.069 \\
\hline \multirow[t]{4}{*}{$\operatorname{Rich}(\phi=1.2)$} & 0.005 & 0.016 & 0.063 \\
\hline & \multicolumn{3}{|c|}{ Large Vent $\left(132.7 \mathrm{~cm}^{2}\right)$} \\
\hline & \multicolumn{2}{|c|}{ Single Cylinder } & Extended Cylinder \\
\hline & Center Ignition & Rear Ignition & Rear Ignition \\
\hline Lean $(\phi=0.8)$ & 0.003 & 0.012 & 0.015 \\
\hline Stoichiometric $(\phi=1)$ & 0.006 & 0.017 & 0.027 \\
\hline $\operatorname{Rich}(\phi=1.2)$ & 0.005 & 0.007 & - \\
\hline
\end{tabular}


Table 5: Summary of the exponential acceleration rates, $\sigma$.

\begin{tabular}{|c|c|c|c|}
\hline & \multicolumn{3}{|c|}{$\sigma$} \\
\hline & \multicolumn{3}{|c|}{ Small Vent $\left(67.9 \mathrm{~cm}^{2}\right)$} \\
\hline & \multicolumn{2}{|c|}{ Single Cylinder } & Extended Cylinder \\
\hline & Center Ignition & Rear Ignition & Rear Ignition \\
\hline Lean $(\phi=0.8)$ & 75.7 & 28.4 & 54.4 \\
\hline Stoichiometric $(\phi=1)$ & 92.1 & 39.6 & 69.1 \\
\hline \multirow{4}{*}{$\operatorname{Rich}(\phi=1.2)$} & 67.5 & 32.5 & 67.2 \\
\hline & \multicolumn{3}{|c|}{ Medium Vent $\left(86.6 \mathrm{~cm}^{2}\right)$} \\
\hline & \multicolumn{2}{|c|}{ Single Cylinder } & Extended Cylinder \\
\hline & Center Ignition & Rear Ignition & Rear Ignition \\
\hline Lean $(\phi=0.8)$ & 70.8 & 23.1 & 60.6 \\
\hline Stoichiometric $(\phi=1)$ & 91.7 & 36.8 & 76.4 \\
\hline \multirow[t]{4}{*}{$\operatorname{Rich}(\phi=1.2)$} & 39 & 29.5 & 64.7 \\
\hline & \multicolumn{3}{|c|}{ Large Vent $\left(132.7 \mathrm{~cm}^{2}\right)$} \\
\hline & \multicolumn{2}{|c|}{ Single Cylinder } & Extended Cylinder \\
\hline & Center Ignition & Rear Ignition & Rear Ignition \\
\hline Lean $(\phi=0.8)$ & 71.4 & 31.2 & 57.6 \\
\hline Stoichiometric $(\phi=1)$ & 88.3 & 32.3 & 69.8 \\
\hline $\operatorname{Rich}(\phi=1.2)$ & 78.5 & 24.9 & - \\
\hline
\end{tabular}

\subsubsection{Comparison of Experiments with the EVA}

The EVA model was updated according to the experiment conditions for comparison. Figure 40 presents both the experiment and the EVA predictions for centrally ignited stoichiometric methane explosion with small vent $A_{v}=67.9 \mathrm{~cm}^{2}$. It was observed that for this condition the EVA predicted the pressure rise during the explosion process between the time of ignition and pressure peak, i.e. $t=0 \sim 0.02 \mathrm{sec}$, very well. However, slightly higher maximum overpressure in this condition was predicted by the EVA comparing to the experiment. After the pressure peak, the EVA seemed not to give much details of the combustion process, as the pressure started dropping after venting occurred, pressure in the vessel fluctuated from the experiment. Maximum overpressures predicted by the EVA and obtained in the experiment were 0.025 bar-g and 0.019 bar-g, respectively, with the model overpredicting it by $31.6 \%$. Figure 41 presents the experiment and the EVA results for centrally ignited stoichiometric methane explosion with medium vent $\left(A_{v}=86.6 \mathrm{~cm}^{2}\right)$. Similar to the small vent conditions, here the EVA also slightly overpredicted 
the maximum overpressure. The predicted and observed maximum pressure values were 0.016 bar-g and 0.012 bar-g, respectively, with the model overpredicting it by $33.3 \%$. Increase in the vent size resulted in decrease in the maximum overpressure due to the larger amount of venting occurring with increased vent area, reducing a higher-pressure development. The EVA and the experiment showed a good agreement in the pressure rise until the peak pressure. Here, with an increase in the vent size, in the experiment larger fluctuations were observed after peak pressure when the venting dominates over the combustion process.

Figure 42 show the predicted pressure history by the EVA compared to that of the experiment for centrally ignited stoichiometric methane explosion in a cylinder with $A_{v}=132.7 \mathrm{~cm}^{2}$. Here, the maximum overpressures of both EVA prediction and the experiment decreased to almost half of the ones in medium vent condition. Due to the large vent, venting occurred earlier comparing to the medium and small vent experiments. This resulted in the pressure drops starting at lower peak pressures. The EVA predicted the pressure rise during the combustion process very well until the peak pressure. After that point, venting started to decrease the pressure. The EVA results showed this as a sharp decrease in the pressure whereas in the experiment, pressure development was not that monotonic. In the experiment, flame struggled as the venting occurred but, pressure developed at the same time due to the combustion in the chamber. The maximum pressures predicted by EVA and observed in the experiment were 0.007 bar-g and 0.006 bar-g, respectively. Here, the difference between peak pressures was relatively low, $\sim 17 \%$. 


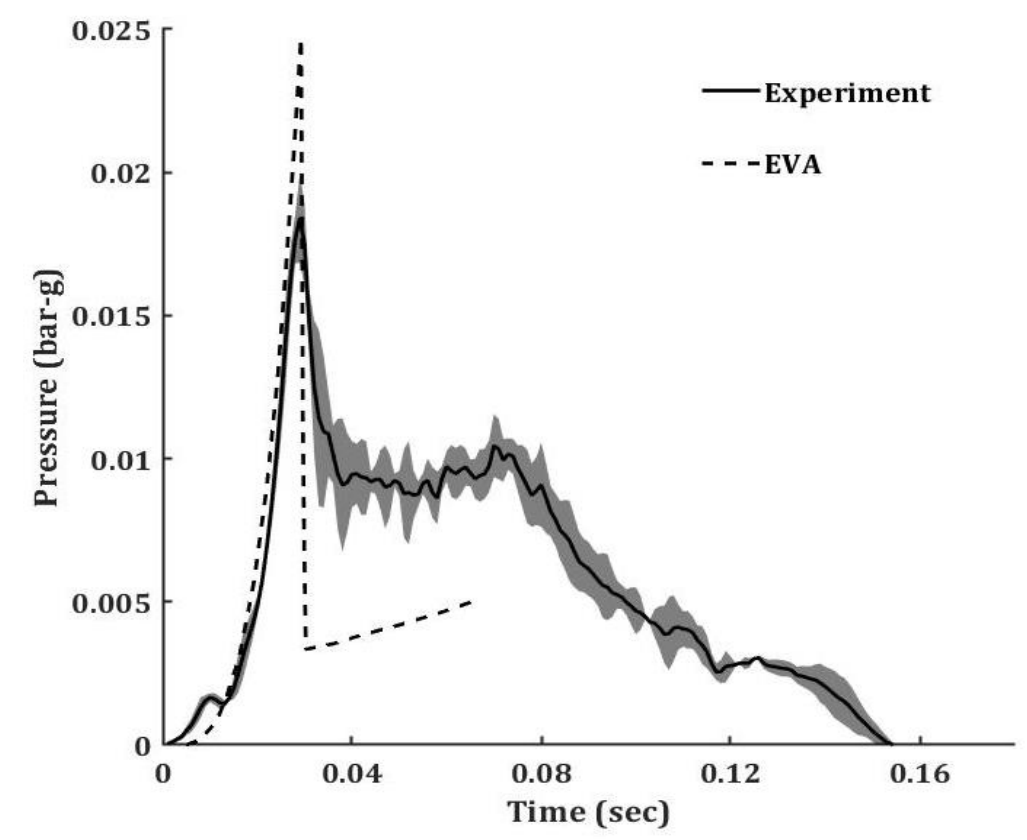

Figure 40: Comparison of experiment with EVA for stoichiometric methane-air explosion in a single cylinder with small vent $\left(A_{v}=67.9 \mathrm{~cm}^{2}\right)$ and center ignition.

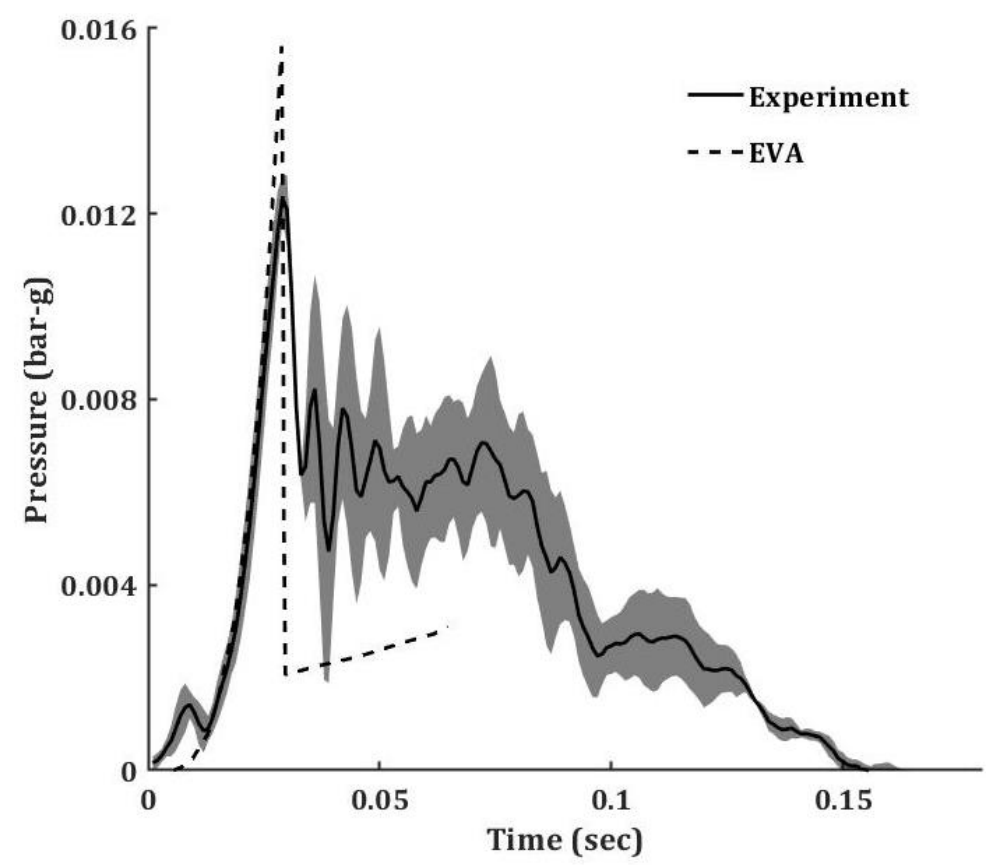

Figure 41: Comparison of experiment with EVA for stoichiometric methane-air explosion in a single cylinder with medium vent $\left(A_{v}=86.6 \mathrm{~cm}^{2}\right)$ and center ignition. 


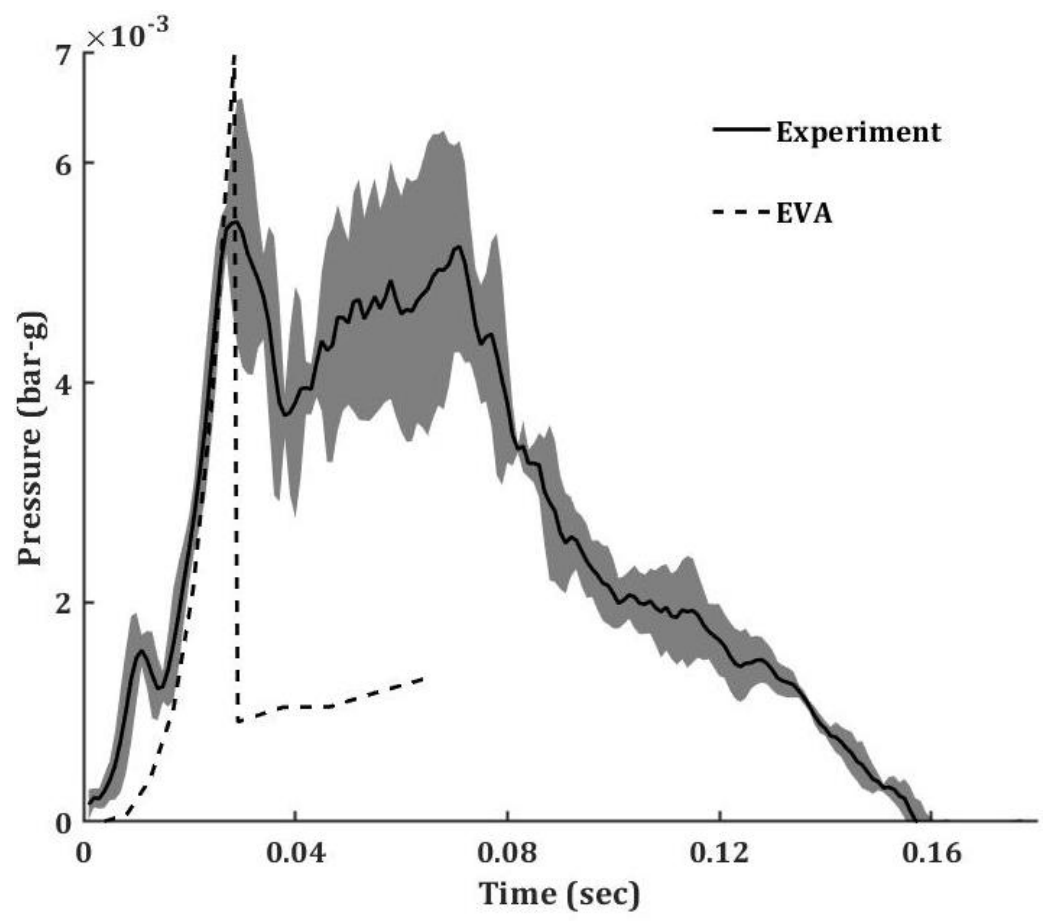

Figure 42: Comparison of experiment with EVA for stoichiometric methane-air explosion in a single cylinder with large vent $\left(A_{v}=132.7 \mathrm{~cm}^{2}\right)$ and center ignition.

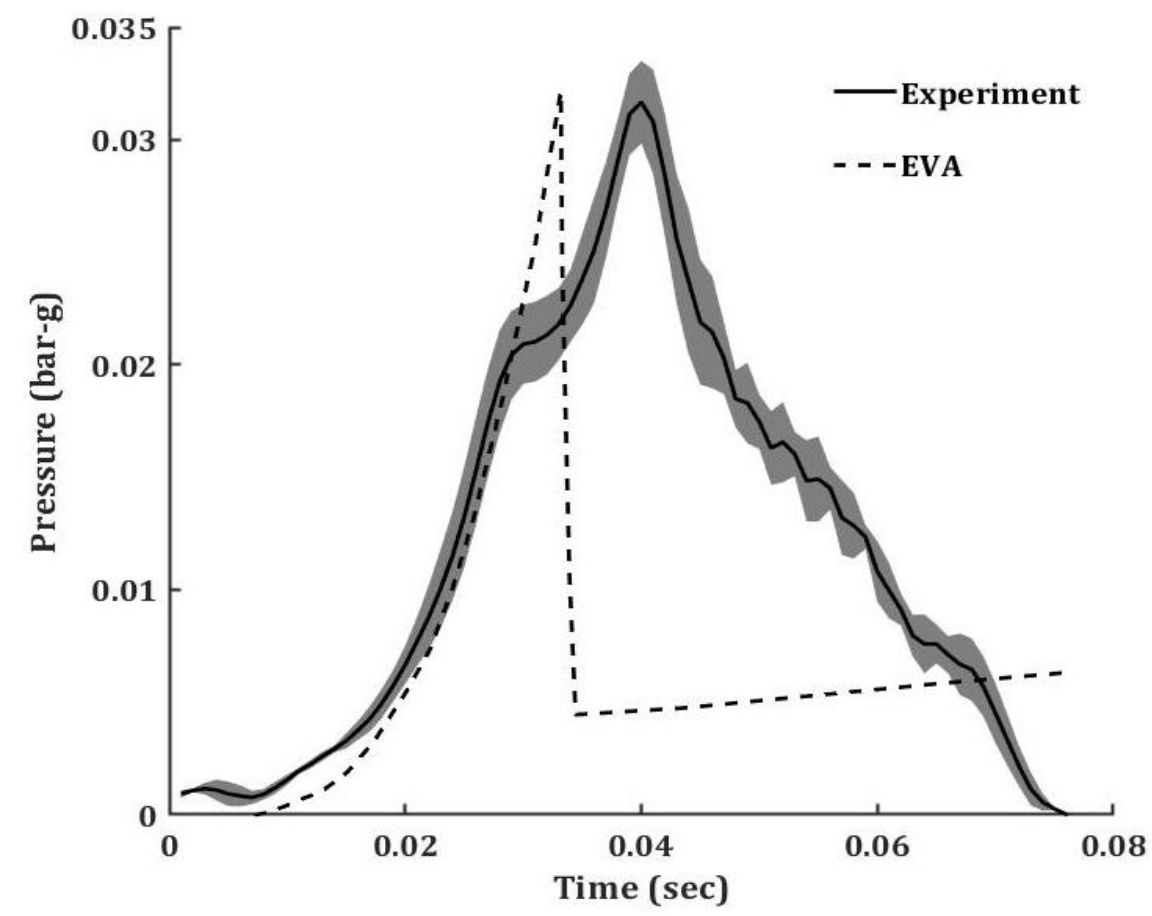

Figure 43: Comparison of rear-ignited stoichiometric methane-air explosion experiment with EVA in a single cylinder with small vent $\left(A_{v}=67.9 \mathrm{~cm}^{2}\right)$.

Next, the EVA predictions were compared to experiment with rear ignition. Figure 43 shows the rear-ignited stoichiometric methane explosion in a cylinder with small vent. The pressure rise and 
drop happened to be smoother in the rear ignition experiments compared to the center ignition experiments. However, the time it took to dissipate all pressure in the vessel after peak pressure was still longer in the experiment compared to the EVA predictions. Pressure rise in the time interval $t=0 \sim 0.03 \mathrm{sec}$, on the other hand, was predicted very well by EVA. However, in the experiment, the pressure decreased from its peak for a short time and then increased again. Results showed that the rear ignition resulted in higher maximum overpressure. This value predicted by EVA was 0.032 bar-g, which was the same in experiments. The difference in time between the peak pressures was $0.007 \mathrm{sec}$, with an earlier preasure peak in the EVA prediction.

Figure 43 show the comparison of rear-ignited stoichiometric methane explosion experiment with the EVA in a cylinder with medium vent. The pressure rise in the experiment was predicted very well by EVA. Similar to the small vent experiment, after the pressure peak, the time it took to decay all pressure inside the chamber was longer in the experiment than in EVA as well. However, the pressure rise of that in the experiment was simulated very well by EVA as well as the maximum overpressure. The maximum overpressures predicted by EVA and obtained in the experiment were 0.021 bar-g and 0.017 bar-g, respectively, with EVA overpredicting it by $23.5 \%$.

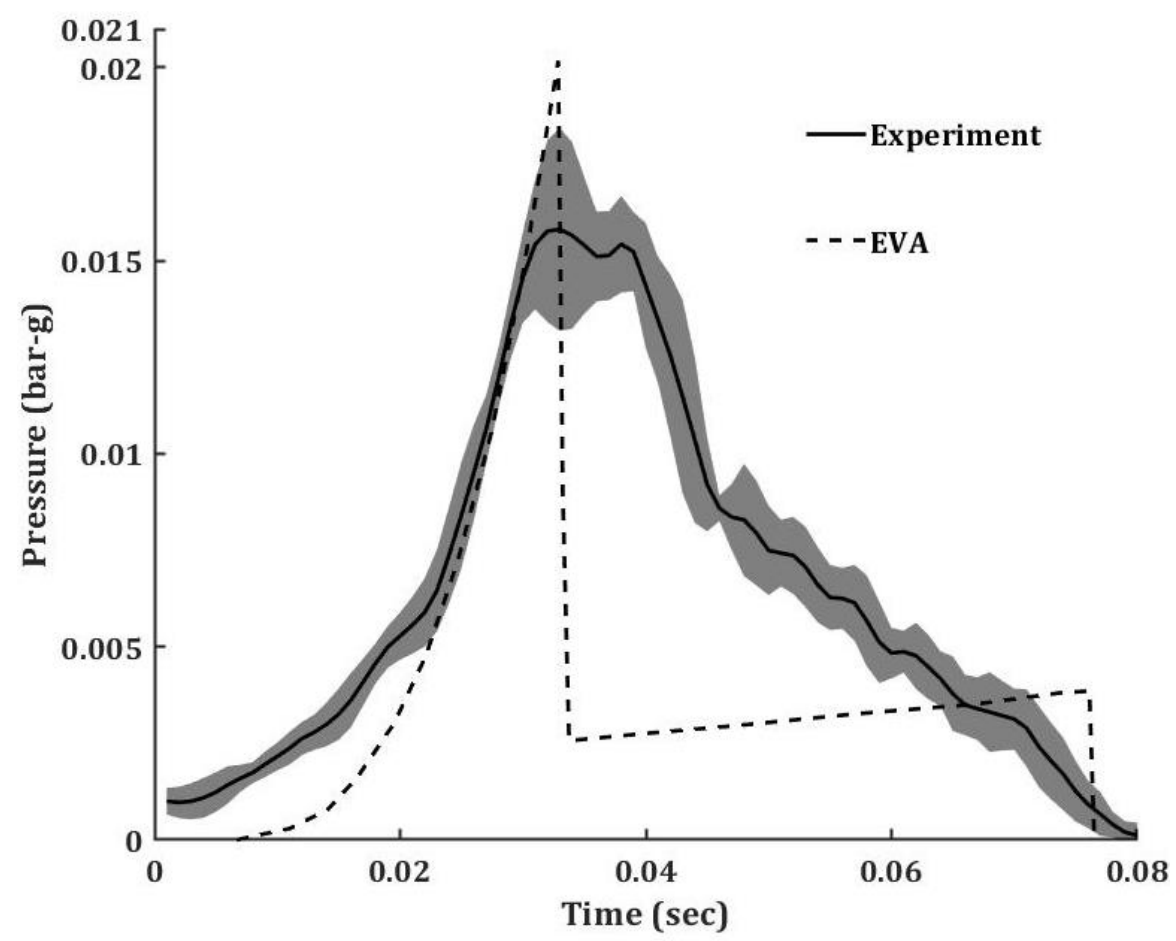

Figure 44: Comparison of rear-ignited stoichiometric methane-air explosion experiment with EVA in a single cylinder with medium vent $\left(A_{v}=86.6 \mathrm{~cm}^{2}\right)$. 
Finally, the pressure history of rear-ignited stoichiometric methane explosion experiment in a single cylinder with large vent was compared to that of the EVA simulation (Fig. 45). Noticeably higher peak pressure was observed in the experiment in this case when compared to EVA results. These peak pressure values were 0.009 bar-g and 0.017 bar-g in EVA simulation and the experiment, respectively. Here, EVA underpredicted the peak pressure with a relatively high difference of $52 \%$. The experiment showed that for rear ignition of stoichiometric methane explosion, increase in the vent to larger size did not prevent high maximum overpressure development. The EVA, in this case, over predicted the venting of gas mixture to the environment compared to the experiment, which prevented the longer pressure rise and resulted in a lower pressure peak.

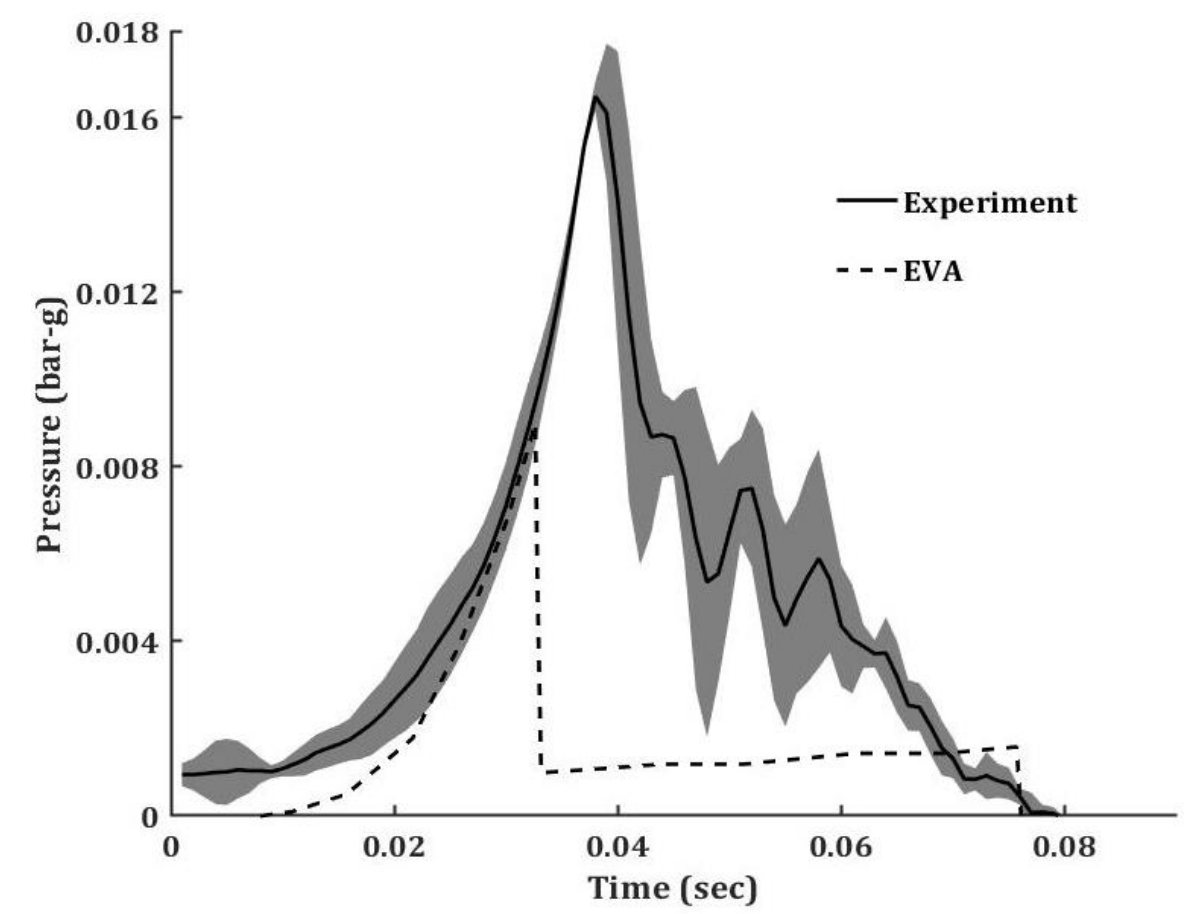

Figure 45: Comparison of rear-ignited stoichiometric methane-air explosion experiment with EVA in a single cylinder with large vent $\left(A_{v}=132.7 \mathrm{~cm}^{2}\right)$.

Overall, the peak pressure obtained from the experiments compared to that predicted by the EVA simulations and the results are shown in Fig. 46 presenting the peak overpressure scaled by the initial pressure versus the vent area $\left(\mathrm{m}^{2}\right)$ for both centrally-ignited and rear-ignited stoichiometric methane explosions. It was seen that the EVA predictions for peak pressure that resulted due to the combustion (deflagration) process of the methane explosions in a vessel with vent panel were in a good agreement with that observed in the experiments for all vent sizes. In all the cases, the 
EVA slightly over-predicted the peak pressure than the experiments, except the case of rearignition with large vent. In the latter condition, higher maximum pressure was obtained in the experiment where the theory seemed to over-predict the venting process.

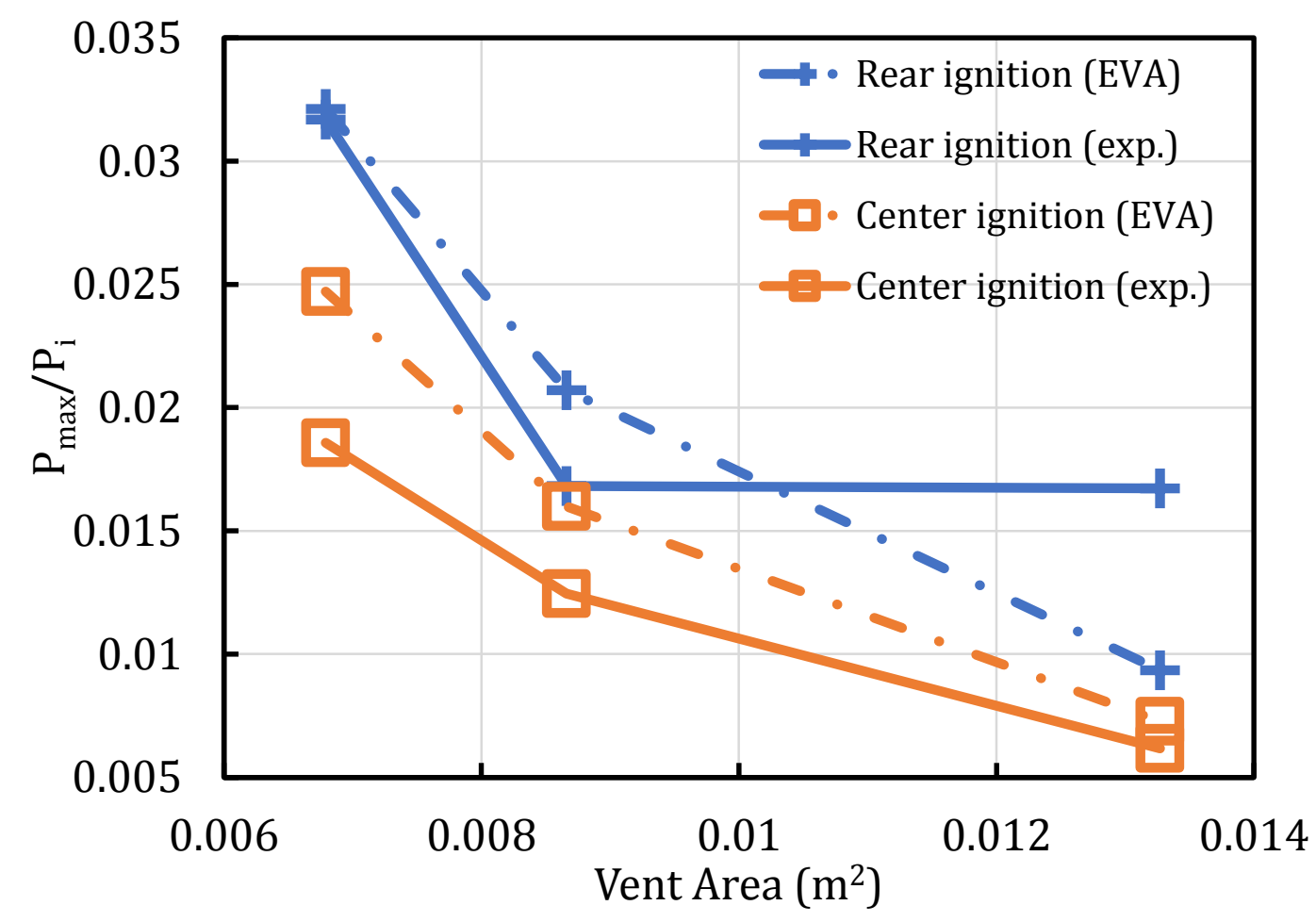

Figure 46: Comparison of peak pressures obtained from the experiments with the EVA.

\subsection{Effect of Wall Conditions on Flame Acceleration at the Early Stages of Burning in Pipes}

In the present subsection, the simulation results obtained for flame acceleration in channels with various wall boundary conditions and flow parameters are discussed. First, both types of mechanistic (free-slip/nonslip) boundary conditions with adiabatic walls are employed to examine the effect of wall friction on flame propagation for various channel half-widths in the range $10 \leq$ $H / L_{f} \leq 30$ and various thermal expansion ratios in the range $5 \leq \Theta \leq 10$. Secondly, both types of the thermal boundary conditions (adiabatic/isothermal) are employed and compared for various wall temperatures in the range $298 \mathrm{~K} \leq T_{w} \leq 600 \mathrm{~K}$, with free-slip walls and the same flow parameters as above - in order to distinguish the influence of heat losses on the flame dynamics and morphology. 


\subsubsection{Impacts of Mechanistic Boundary Conditions}

Figure 47 compares the flame acceleration scenarios in channels of half-width $H=30 L_{f}$ with free-slip, Fig. 47a, and nonslip, Fig. 47b, walls. In both cases, the flame dynamics exhibited a sequence of four stages, namely: (i) a hemispherical flame, (ii) a finger-shaped flame front, (iii) a quasi-planar flame and, eventually, (iv) an inverted concave tulip flame. The first stage occurred immediately after ignition when the flame embryo developed into a small hemispherical front that expanded uniformly outwards. However, the flame front tended to spread in the $x$-direction since expansion in the $z$-direction was limited by the sidewalls. As a result, the flame started elongating. The second stage occurred as a result of such elongation, leading to acquiring of a finger-like flame shape, accompanied by the exponential increase in the flame surface area (length in 2D) as well as in the total burning rate. This finger flame acceleration stopped when the flame skirt, which refers to the side segments of the flame front, contacted the sidewalls of the channel. At the next, third stage, the flame skirt formed small angles with the channel sidewalls; the flame velocity at these points then exceeded the laminar flame velocity, which made the flame skirt catching up the flame tip to form a quasi-planar flame. Finally, at the fourth stage, the flame inverted to form a cusp pointing backward or acquiring a tulip shape. This scenario agrees with the findings of Refs. $[176,180,181]$. 

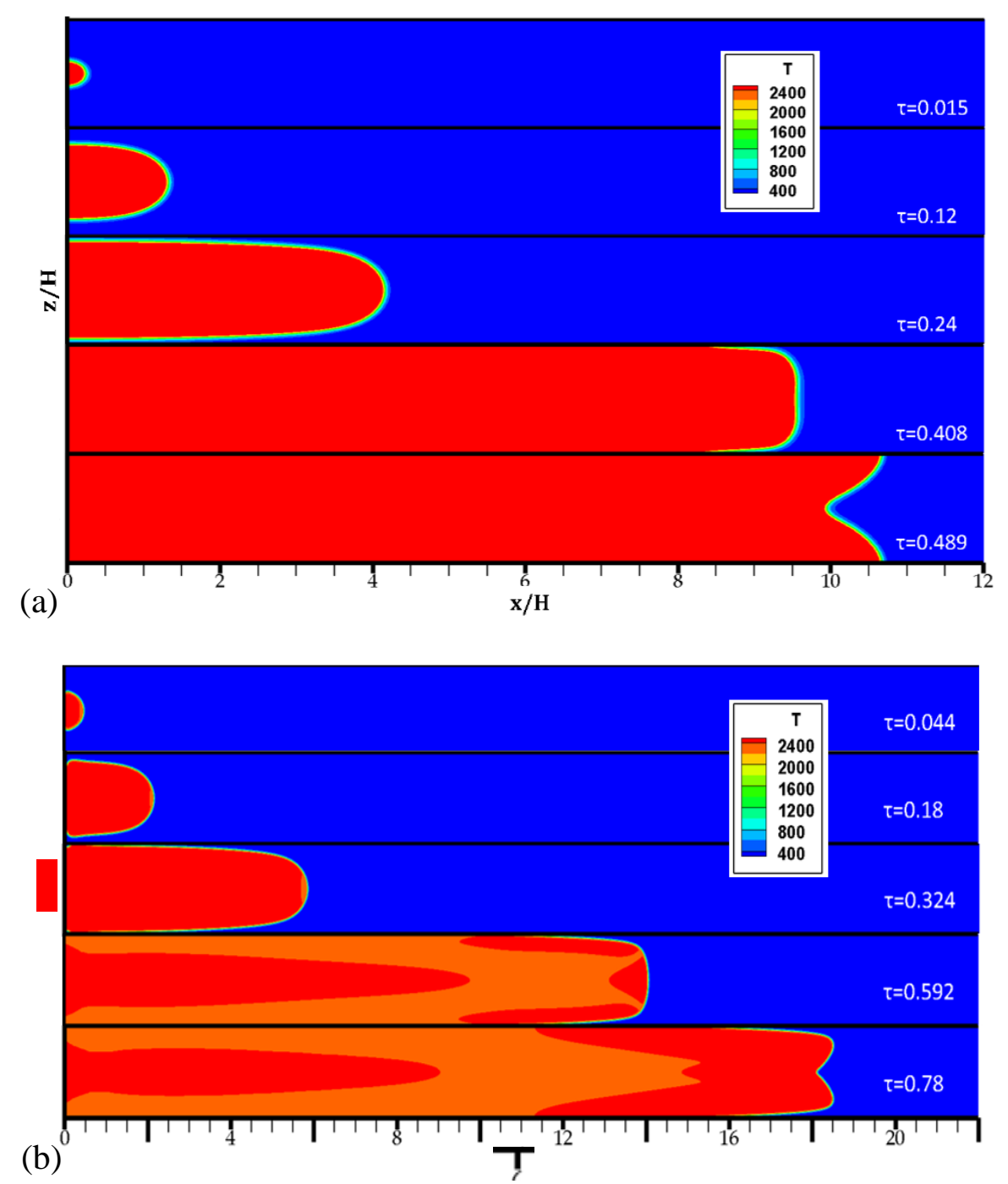

Figure 47: Color temperature snapshots (in $\mathrm{K}$ ) for the evolution of a flame with $\Theta=8$ in a channel of half-width $H / L_{f}=30$ with adiabatic, slip (a) and nonslip (b) walls

As for the differences between the situations of slip and nonslip wall conditions, one of them is a corrugated flame shape at a closed end occurring due to wall friction at a nonslip wall. It is further noted that the temperature evolution was uniform during flame propagation for the slip walls, while some perturbations were seen for nonslip walls, when the flame front contacted a sidewall of the channel. Moreover, as the flame approached the sidewalls, the effect of wall friction provided a corrugated tulip flame at the instant $\tau=t U_{f} / R=0.78$; see the case of nonslip walls in Fig. $47 \mathrm{~b}$. 

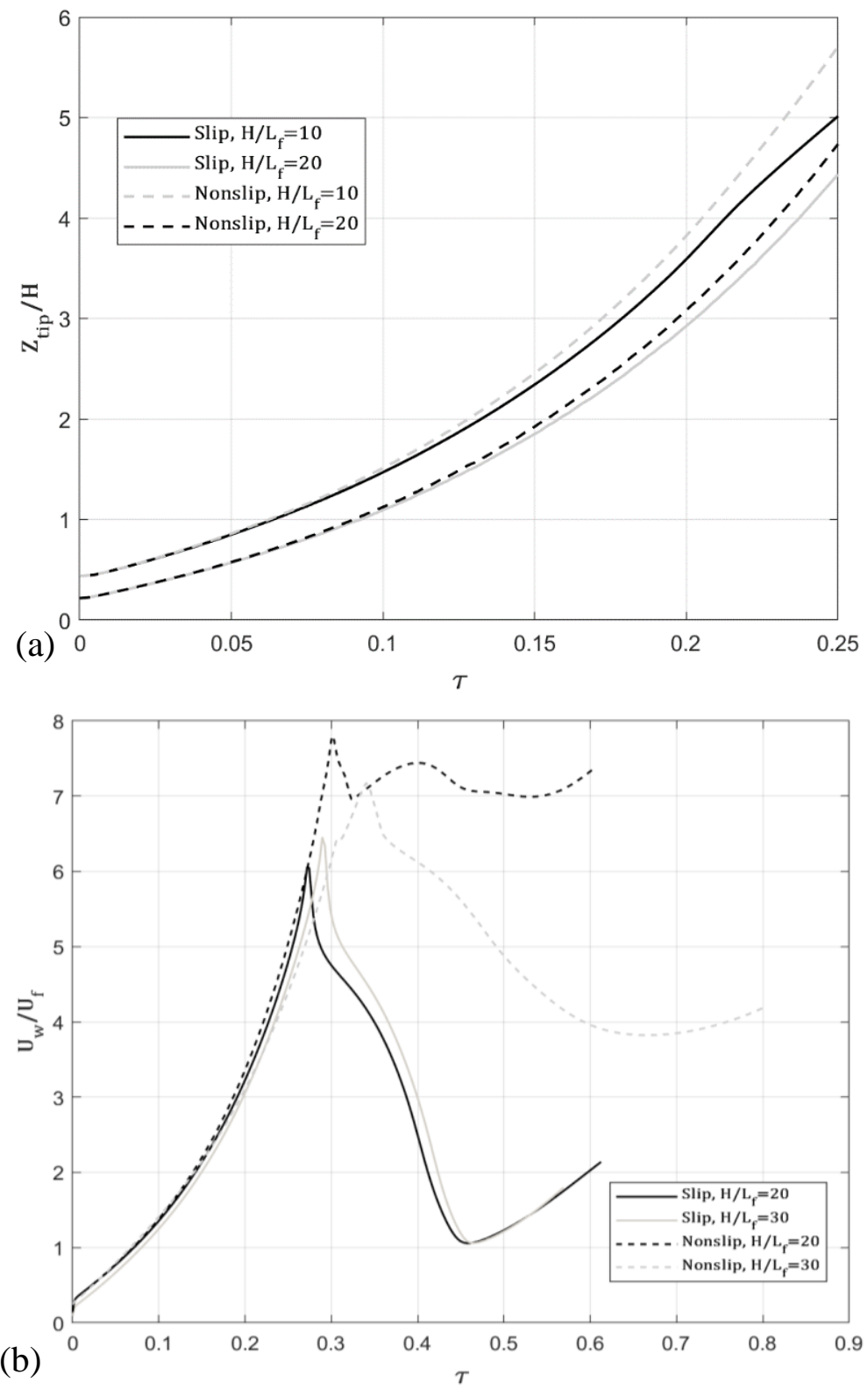

Figure 48: The scaled flame tip position $Z_{\text {tip }} / H$ (a) and the scaled total burning rate $U_{w} / U_{f}$ (b) versus the scaled time $\tau=U_{f} t / H$ for slip (solid) and nonslip (dashed) adiabatic walls for various channel widths.

To quantify the qualitative, visual findings of Fig. 47, in Fig. 48 the time evolution of the scaled flame tip position $Z_{\text {tip }} / H$, Fig. 48 a (during the acceleration stage only), and that of the scaled total burning rate $U_{w} / U_{f}$, Fig. 48b, are presented for the channels of various half-widths in the cases of both slip and nonslip walls. It is seen in Fig. 48a that the effect of wall friction was minor (within the accuracy of the numerical approach) during the acceleration stage, when a flame front acquired a finger shape. This can be explained by the fact that acceleration occurred at the centerline, i.e. away from the channel sidewalls. However, according to Fig. 48b, as the flame skirt came to a 
sidewall, in the case of nonslip walls, wall friction resulted in a noticeable increase in the total burning rate as compared to the event of free-slip walls, when the total burning rate decreased sharply.

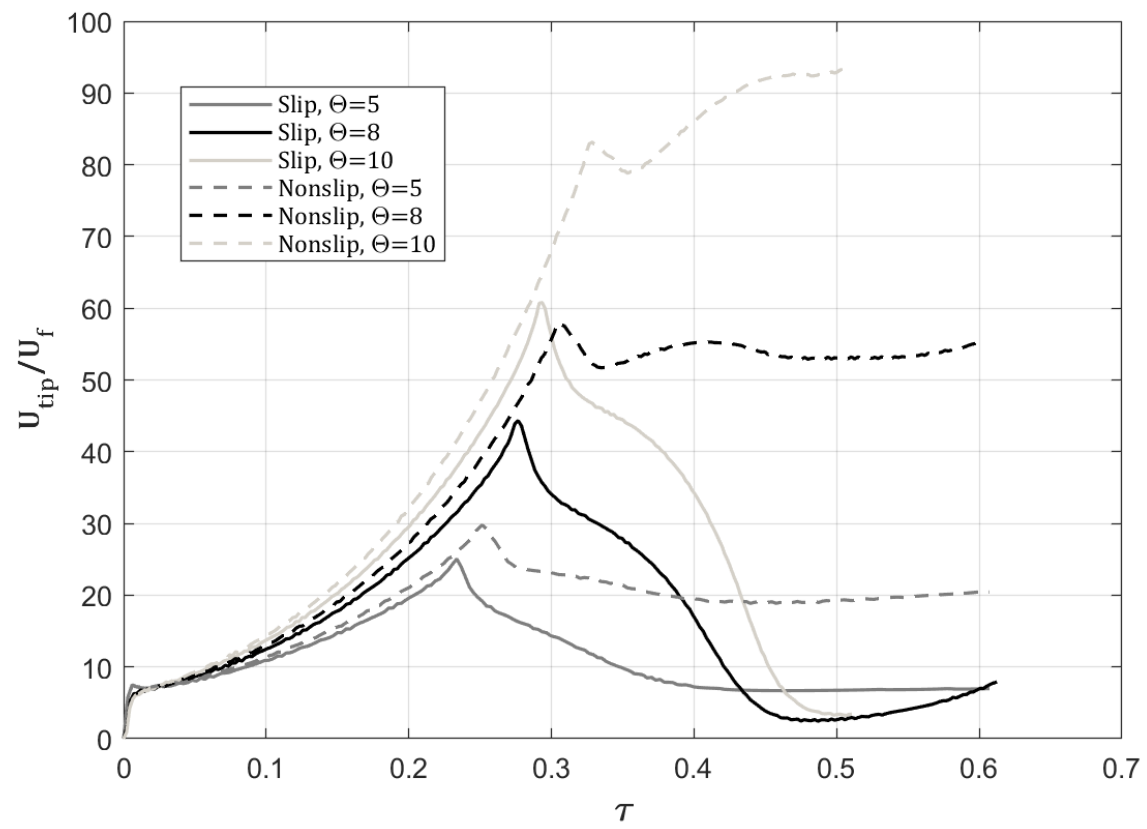

Figure 49: The scaled flame tip velocity, $U_{t i p} / U_{f}$, versus the scaled time $\tau=U_{f} t / H$ for the slip (solid) and nonslip (dashed) adiabatic walls with various thermal expansion ratios $\Theta=5,8,10$.

Next, the impact of such a key factor of premixture as thermal expansion in the burning process on finger flame acceleration was analyzed. Specifically, numerical simulations were performed for various thermal expansion ratios, $\Theta=5,8$ and 10, and fixed channel half-width $H=20 \mathrm{~L}_{f}$, with both slip and nonslip adiabatic walls employed. The result is shown in Fig. 49, where the time evolutions of the scaled flame tip velocity $U_{t i p} / U_{f}$ were compared for all these cases, with the solid and dashed lines devoted to the slip and nonslip walls, respectively. All the curves of Fig. 49 exhibited similar qualitative trends, with initial expansion of a hemispherical flame, followed by elongation of a convex finger-shaped front and ending with formation of a concave, tulip flame. The flame tip velocity increased with the thermal expansion ratio for both cases of free-slip and nonslip walls. Furthermore, the flame shape was more corrugated in the presence of wall friction and, therefore, the maximal flame tip velocity in the case of nonslip walls was higher than that in the event of slip walls. The maximal values for the total burning rate and the scaled flame tip velocity for slip and nonslip walls were also summarized in Table 6. 
Table 6: The maximal scaled flame tip velocity and the maximal scaled total burning rate for different thermal expansion coefficients.

\begin{tabular}{|cccccc|}
\hline B.C & $\Theta$ & $\boldsymbol{U}_{\text {tip, max }} / \boldsymbol{U}_{\boldsymbol{f}}$ & $\boldsymbol{\tau}_{\boldsymbol{U}_{\text {tip } \boldsymbol{p}, \boldsymbol{m a x}}}$ & $\boldsymbol{U}_{\boldsymbol{w}, \boldsymbol{m a x}} / \boldsymbol{U}_{\boldsymbol{f}}$ & $\boldsymbol{\tau}_{\boldsymbol{U}_{\boldsymbol{w}}, \boldsymbol{m a x}}$ \\
Slip & 5 & 25.1 & 0.234 & 5.53 & 0.231 \\
Nonslip & 5 & 29.8 & 0.251 & 7.04 & 0.248 \\
Slip & 8 & 44.3 & 0.277 & 6.06 & 0.272 \\
Nonslip & 8 & 58.0 & 0.302 & 7.80 & 0.304 \\
Slip & 10 & 60.8 & 0.294 & 6.83 & 0.289 \\
Nonslip & 10 & 93.5 & 0.510 & 10.5 & 0.510 \\
\hline
\end{tabular}

\subsubsection{Impacts of Thermal Boundary Conditions}

In order to examine the effect of thermal boundary conditions on the combustion process, the adiabatic as well as the isothermal walls of various temperatures are next employed and compared. The color temperature snapshots of Fig. 50 represent the typical finger flame evolution scenarios in a channel of half-width $H=20 L_{f}$ with slip, isothermal walls. Specifically, Fig. 50a is devoted to the "cold" walls kept at the room temperature, $T_{w}=298 \mathrm{~K}$, while the flame evolution in a channel with preheated walls, $T_{w}=600 \mathrm{~K}$, is presented in Fig. 50b. According to Fig. 50, a hemispherical flame expanded until the flame skirt contacted the sidewalls and the heat loss cooled down the flame such that the flame surface area and the total burning rate then decreased dramatically. A tulip flame was formed, followed by its transition into a quasi-planar flame, which then collapsed to acquire a curved convex shape, presumably, controlled by the wall temperature.

The qualitative difference between the adiabatic and isothermal wall conditions can be seen when comparing Fig. 47a, devoted to the slip adiabatic walls, to Figs. 50 (a, b), representing the slip isothermal wall conditions. Specifically, when the combustor walls were kept at a room temperature, Fig. 50a, the heat exchange occurred when the burnt matter expanded, and heat was transferred from the combustor to the environment through the cold walls. The heat loss caused the flame skirt to cool down where the region of the heat was then localized around the flame tip. The same effect is seen in Fig. 50b, for the preheated walls, where the temperature of the burnt matter decreased. In contrast, a uniform temperature evolution for flame propagation with adiabatic walls was observed, because heat was conserved at the combustor walls in that case. 


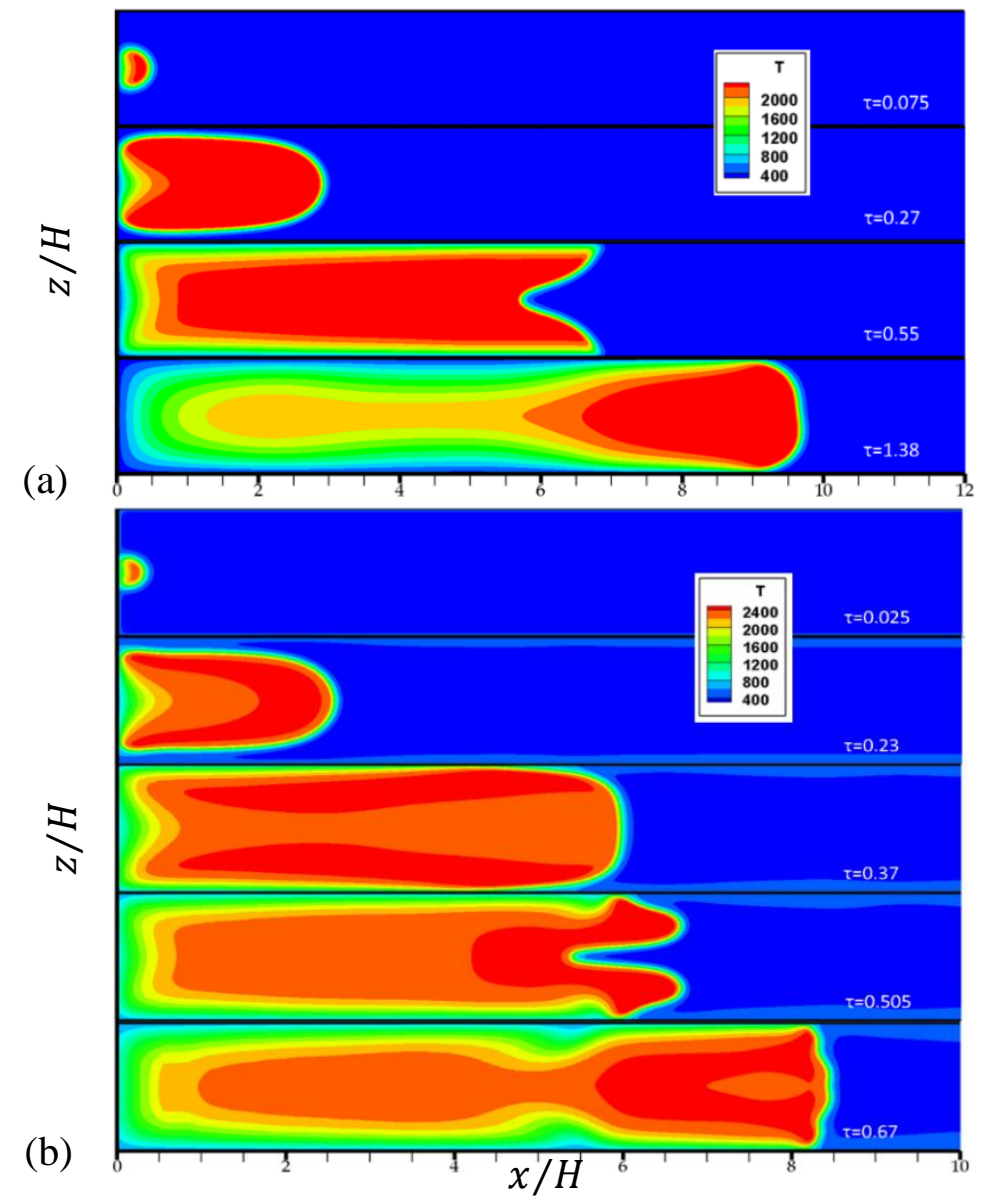

Figure 50: The temperature snapshots for the evolution of a flame with the thermal expansion ratio $\Theta=8$ propagating in a channel of half-width $H / L_{f}=20$ with the slip, isothermal walls: (a) cold, $T_{w}=298 \mathrm{~K}$ and (b) preheated, $T_{w}=600 \mathrm{~K}$.

A quantitative difference between the flame propagation scenarios in adiabatic and isothermal channels is demonstrated in Fig. 51, where the scaled total burning rates $U_{w} / U_{f}$ are compared for various thermal wall conditions. It is seen that the thermal boundary conditions had a minor effect on flame acceleration: it weakened but only slightly with the reduction of the wall temperature. This also can be explained by the fact that finger flame acceleration occurred away from the sidewalls and the heat loss effect emerged only when the flame skirt approached the sidewalls. 


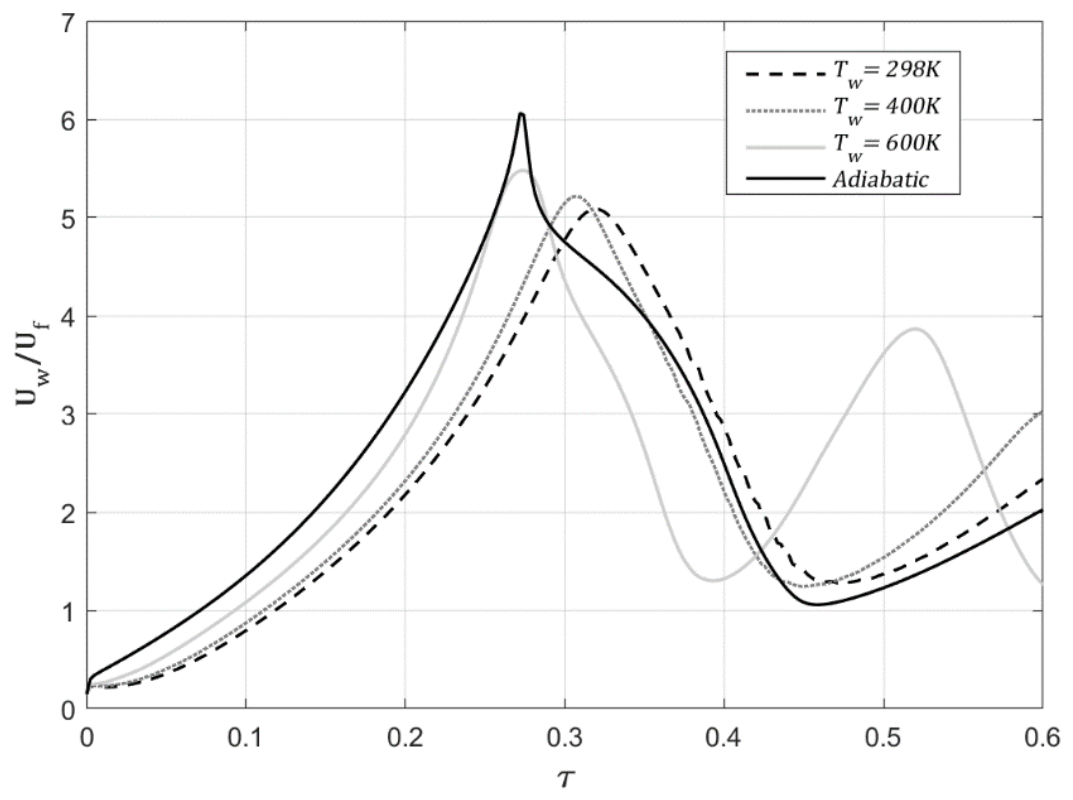

Figure 51: The scaled total burning rate, $U_{w} / U_{f}$, versus the scaled time $\tau=U_{f} t / H$ for a flame with the thermal expansion ratio $\Theta=8$ propagating in a channel of half-width $H / L_{f}=20$ with adiabatic walls (solid) and isothermal walls (dashed) at various wall temperatures $T_{w}=298,400,600 \mathrm{~K}$.

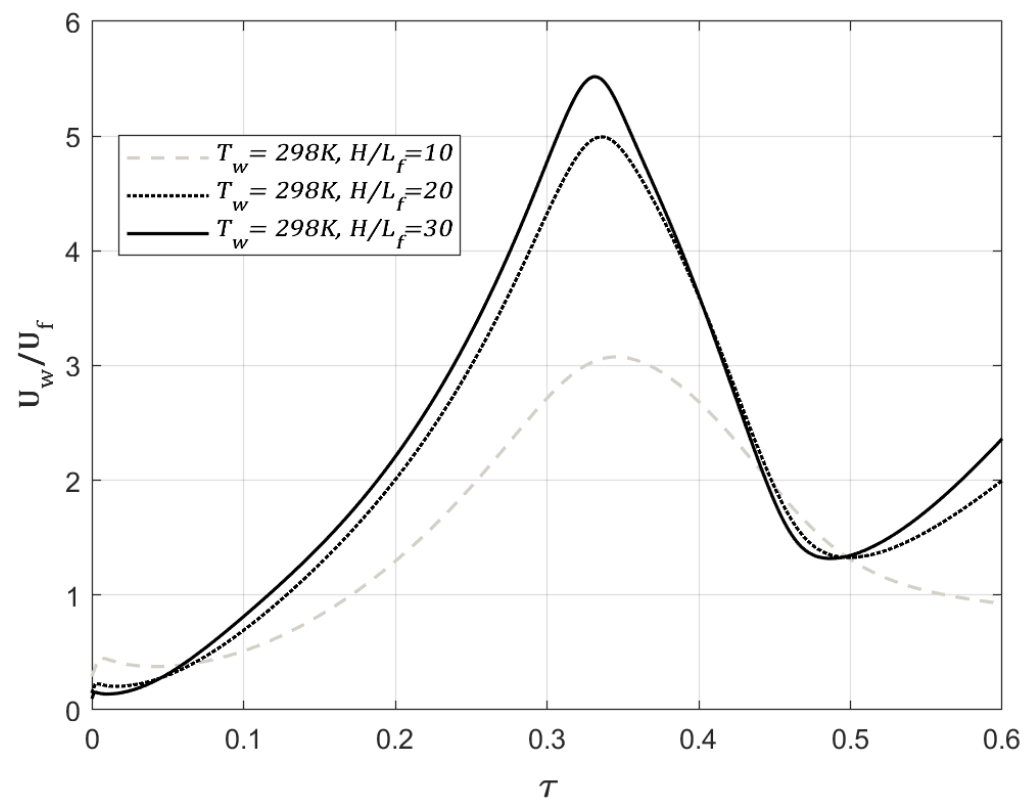

Figure 52: The scaled total burning rate, $U_{w} / U_{f}$, versus the scaled time $\tau=U_{f} t / H$ for a flame with the thermal expansion ratio $\Theta=8$ propagating in the channels of half-widths $H / L_{f}=10,20,30$ with slip, isothermal cold walls, $T_{w}=298 \mathrm{~K}$.

Next the impact of the channel size for isothermal walls was investigated, where it is expected to play a better role as compared to the adiabatic channels. For this purpose, Fig. 52 compares $U_{w} / U_{f}$ for various channel half-widths in the range $10 \leq H / L_{f} \leq 30$ in particular case of a room $\left(T_{w}=\right.$ 
$298 \mathrm{~K}$ ) wall temperature. It is seen that the role of the channel width was noticeable for cold walls kept at the initial fuel temperature, especially as the channel size decreases to $H=10 L_{f}$, while the effect was minor as $H$ grew from $20 L_{f}$ to $30 L_{f}$. Moreover, the impact of the channel width on the acceleration rate was minor, since the flame approached the sidewalls almost at the same scaled time instants $\tau \approx 0.32$.

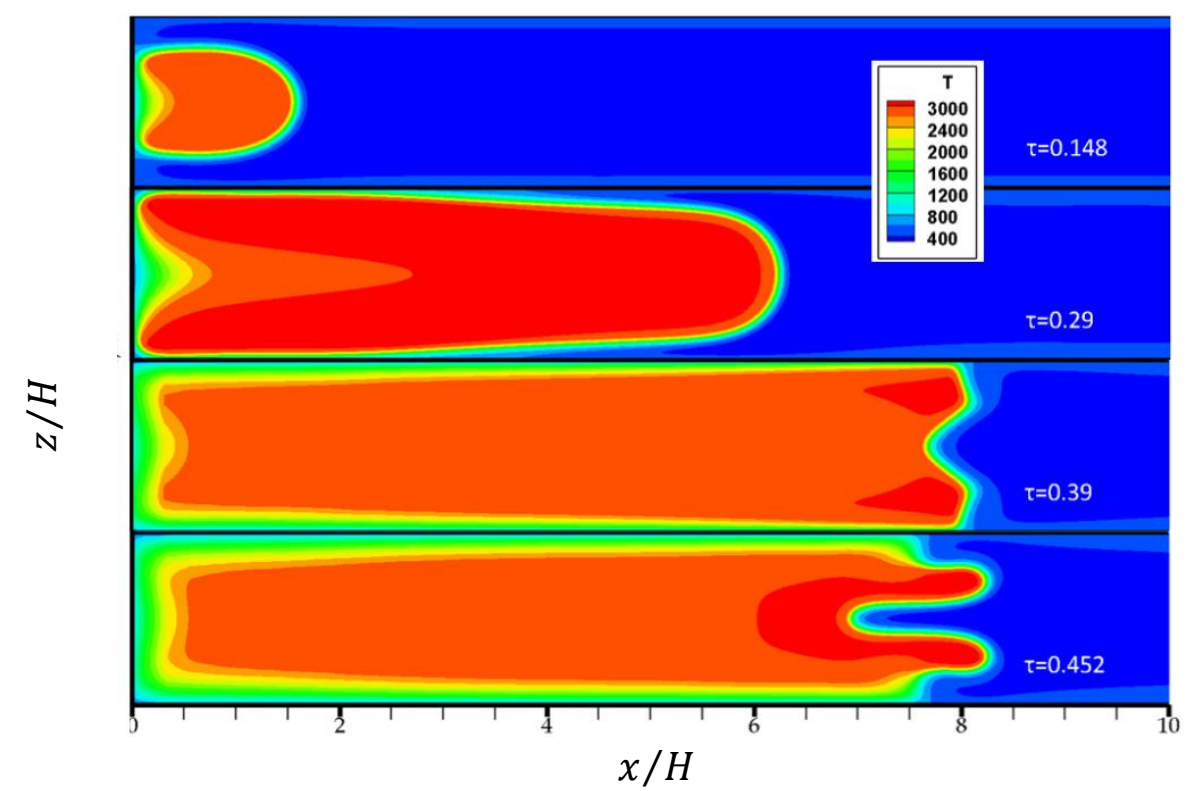

Figure 53: The color temperature snapshots for the evolution of a flame with the thermal expansion ratio $\Theta=10$ in a channel of half-width $H / L_{f}=20$ with the slip, isothermal (preheated) walls at $T_{w}=600 \mathrm{~K}$.

Next the impact of thermal expansion on flame propagation was discussed at various thermal boundary conditions. Specifically, Fig. 53 shows the impact of a relatively high thermal expansion ratio, $\Theta=10$, on a flame propagating in an isothermal $\left(T_{w}=600 \mathrm{~K}\right)$ channel of half-width $H=$ $20 L_{f}$. Overall, the flame dynamics was qualitatively the same as in the previous cases: the standard finger flame scenario was seen, with initial expansion of a hemispherical flame front, followed by acceleration of a finger flame and then deceleration and formation of a tulip flame. The impact of a higher thermal expansion resulted in a more corrugated tulip flame, which experienced higher distortion of the front as the flame swept on the sidewalls. 


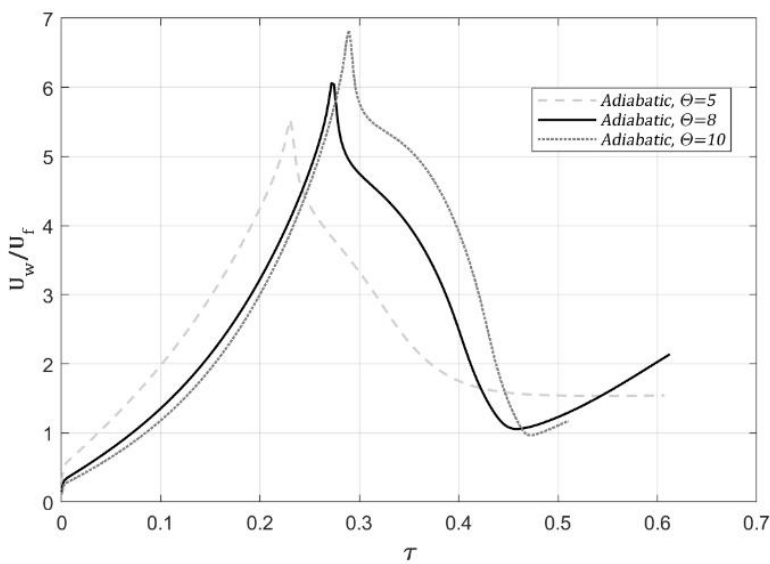

(a)

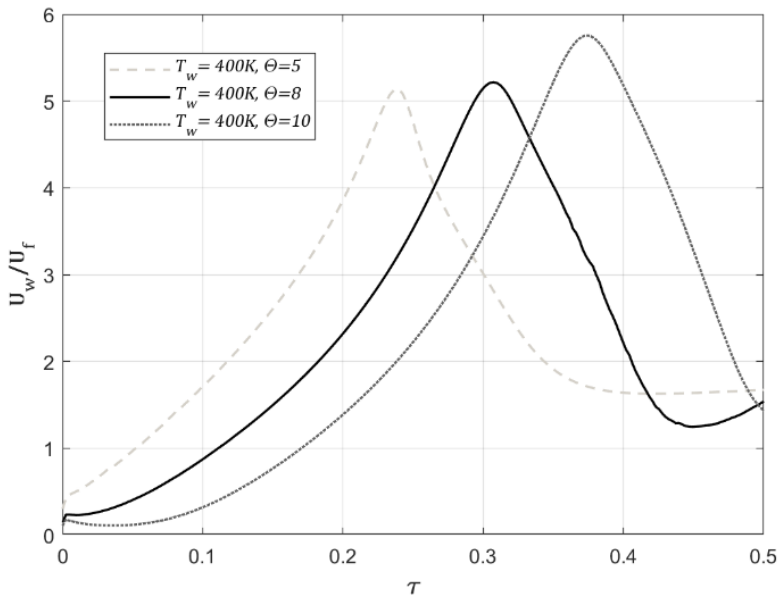

(b)

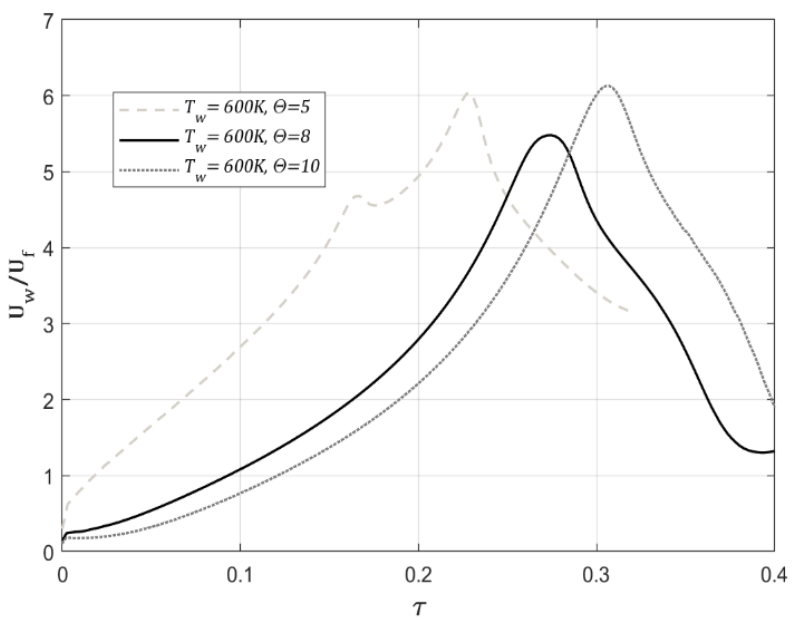

Figure 54: The scaled total burning rate, $U_{w} / U_{f}$, versus the scaled time $\tau=U_{f} t / H$ for the flames of various thermal expansion ratios $\Theta=5,8,10$ propagating in a channel of half-width $H / L_{f}=20$ with adiabatic walls (a) and preheated isothermal walls with the wall temperatures $T_{w}=400 \mathrm{~K}$ (b) and $600 \mathrm{~K}(\mathrm{c})$.

Finally, Fig. 54 shows the evolution of the scaled total burning rate $U_{w} / U_{f}$ versus the scaled time $\tau$ for the adiabatic, Fig. 54a, as well as isothermal preheated walls at constant temperatures of 
$400 \mathrm{~K}$, Fig. 54b, and $600 \mathrm{~K}$, Fig. 54c. Here, the channel half-width is same, $H=20 L_{f}$, but various thermal expansion ratios in the range $5 \leq \Theta \leq 10$ in all cases. It is seen that the maximal burning rate slightly increased with $\Theta$ for adiabatic walls, while the effect was minor in the case of isothermal walls. Furthermore, thermal expansion had an impact on flame acceleration as a flame approached the sidewalls faster with lower $\Theta$ for both thermal boundary conditions, especially for isothermal walls. With these results, the analysis on the effect of wall conditions on finger-like flame acceleration scenario was concluded.

\subsection{Analytical Study of a Burning Accident in an Obstructed Coalmining Passage}

Here, the obstacles are imitated by a comb-shaped (Bychkov) array, Fig. 55, since it is simple to study and is known to provide extremely powerful flame acceleration [206]. Specifically, the formulation of a globally-spherical self-accelerating expanding flame front, distorted by the DL instability [212], the theory of finger flame acceleration occurring at the initial stages of burning in a pipe [180], and the formulation of ultrafast flame acceleration due to obstacles in an obstructed tube/channel [207] are combined, thus revisiting a predictive scenario of a burning accident in a coalmining passage [182]. It is shown that the obstacles may play the key role in the coalmining fire scenario, with stronger effect seen in cylindrical geometry, and that the DL instability may significantly promote obstacles-based acceleration. 

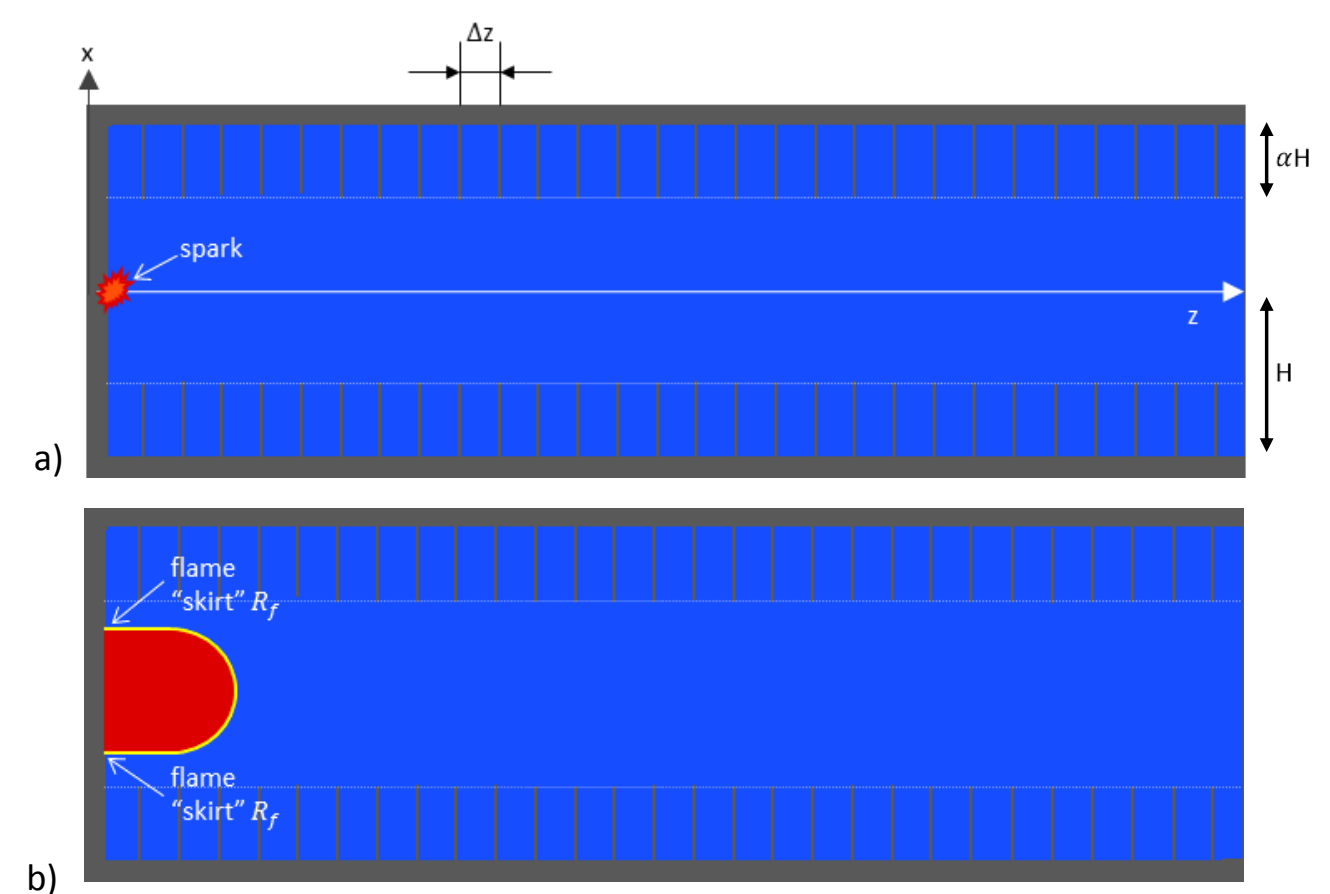

b)
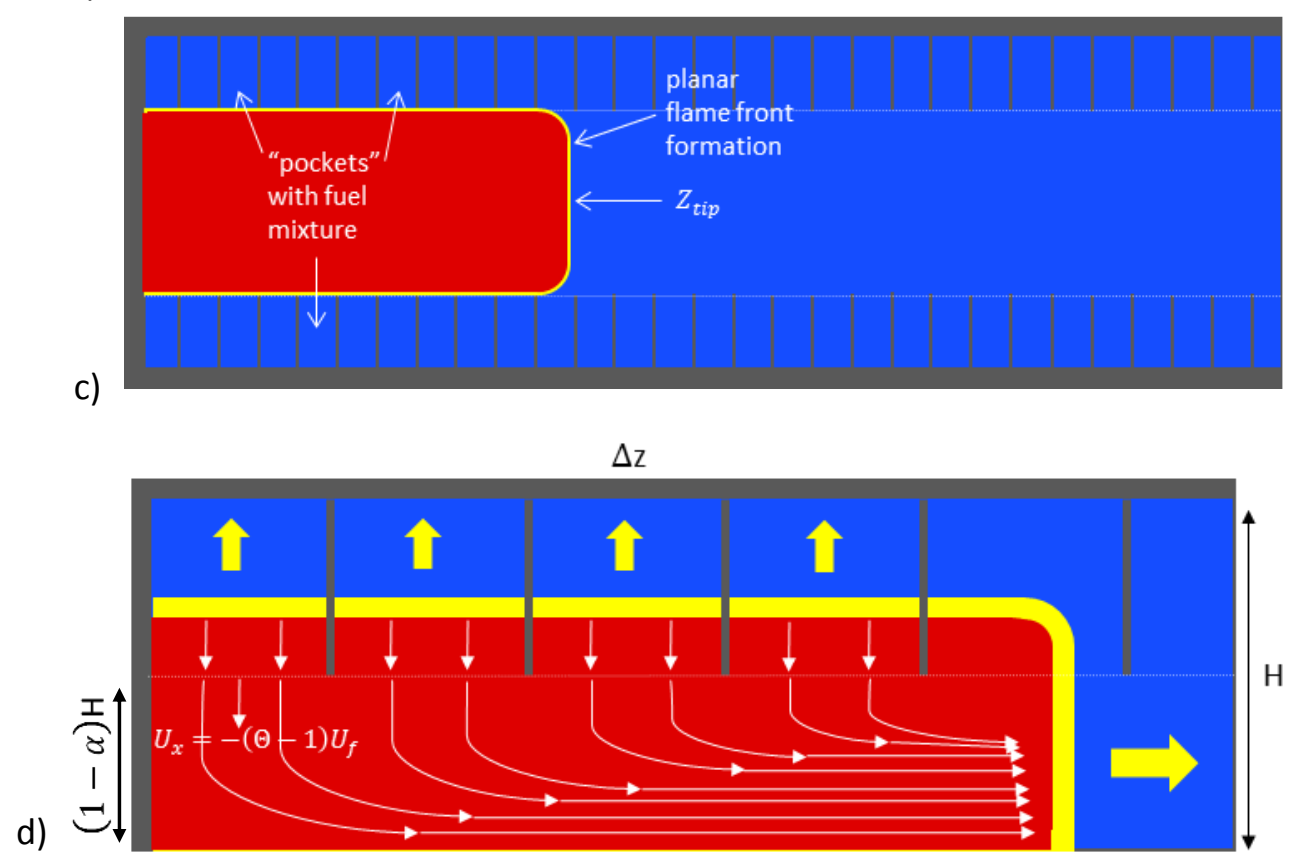

Figure 55: An illustration of the Bychkov mechanism of flame acceleration in an obstructed passage [206].

Starting with homogeneous methane-air fuel mixture, we subsequently extend the analysis to a gaseous-dusty environment using a modified Seshadri formulation [182,202]. The parametric study includes the blockage $(0 \leq \alpha \leq 2 / 3)$ and equivalence ratios $(0.6 \leq \phi \leq 1.4)$ as well as the 
size of the dust particles $\left(r_{s}=10,75 \mu \mathrm{m}\right)$ and their type (inert; such as sand, combustible; i.e. coal, and their combination).

\subsubsection{Formulation in 2-D Passage (Channel)}

First, a 2D passage (channel) of width $2 H=2.1 \mathrm{~m}$ was considered as illustrated in Fig. 55, which is closed at one end, and a premixed flame propagates towards the open end. The passage is blocked by obstacles of length $\alpha H$ such that the channel along the central part $(1-\alpha) R$ is unobstructed. From the ignition time and until a time instant when a flame "skirt" touches an obstacle, $t_{o b s}$, the flame evolution is described by a predictive scenario of a burning accident in an unobstructed coalmining passage [182], which combines the mechanism of flame acceleration caused by a finger flame shape (see Ref. [180] for details), with that of a globally-spherical, expanding flame front corrugated due to the DL instability [212]. Here, the basics of the latter acceleration mechanism [212], devoted to self-similar accelerative outward propagation of a globally-spherical flame front, which is wrinkled because of the Darrius-Landau (DL) instability (see Ref. [212] for more details on the dynamic and scalar fields associated with flame propagation and the fluid ahead of the flame front), as well as of its combination with the finger flame acceleration mechanism [182] are briefly recalled. Any large-scale premixed flame front is prone to the DL instability. In particular, the radius of a globally-spherical expanding flame obeys the time-dependent power law, $R_{f}(t)=R_{0}+C t^{n}$, where a small quantity $R_{0}$ can be considered as a fitting parameter that may account for the ignition uncertainty in the experiments or a critical flame radius at which self-similar acceleration visually starts [212]. In the present work, we neglect this parameter as negligible compared to a passage height or an obstacle length, $R_{0} \ll \alpha H<H$. The factor $C$ can be evaluated as $C=k_{D L}^{n-1}\left(\Theta U_{f} / n\right)^{n}$ and with $n=1.3 \sim 1.5$ [212], such that instead of the unstretched laminar burning velocity $U_{f}$, the instantaneous radial flame velocity with respect to the fuel mixture is $[182,212]$

$$
U_{D L}(t)=U_{f}\left(\frac{\Theta}{n} k_{D L}\right)^{n-1} t^{n-1}, \quad k_{D L}=L_{f}^{-1}\left\{1+\frac{(\Theta-1)}{(\Theta-1)^{2}} \Theta \ln \Theta\right\}^{-1},
$$

where $k_{D L}=2 \pi / \lambda_{D L}$ is the DL cut-off wavenumber with $\lambda_{D L}$ being the DL critical wavelength, $\lambda_{D L}=2 \pi L_{f}\left(1+\frac{(\Theta+1)}{(\Theta-1)^{2}} \Theta \ln \Theta\right)$. In this dissertation work, this equation for $\lambda_{D L}$ is chosen because it was used in the previous works such as Ref. [182], so it is natural to use the same formula here, 
for better comparison. It is nevertheless noted that there exist alternative formulas for $\lambda_{D L}$ as discussed in Ref. [213] and references therein. In this light, the present formulation is flexible with respect to a choice of the formula for $\lambda_{D L}$, such that the equation for $\lambda_{D L}$ can be readily replaced by any alternative. The DL cutoff wavelength $\lambda_{D L}$ is sometimes considered as an "effective" flame thickness [213]. This is because the internal flame structure (i.e. the finite flame thickness $L_{f}$ ) should be considered at the length scales smaller and comparable to the DL cutoff ( $\lambda \ll \lambda_{D L}$, $\left.\lambda \leq \lambda_{D L}, \lambda \sim \lambda_{D L}\right)$; while at much larger scales $\left(\lambda \gg \lambda_{D L}\right)$ the internal flame structure can be neglected, so the flame front can be considered as infinitely thin $\left(L_{f} \rightarrow 0\right)$. In fact, that is why the DL cutoff wavelength is a quantity, proportional to the flame thickness, $\lambda_{D L} \propto L_{f}$, with a proportionality factor of the order of $10^{1} \sim 10^{2}$ depending on thermal-chemical flame properties such as the expansion ratio $\Theta[213,214]$. The equation for $\lambda_{D L}$ described above is an example of such a dependence. Obviously, if $L_{f} \rightarrow 0$ then $\lambda_{D L} \rightarrow 0$.

In Ref. [212], the authors developed an analytical theory describing self-similar accelerative outward propagation of a globally-spherical flame front, which is wrinkled because of the DL instability and obeys Eq. (4.1). In particular, the dynamic and scalar fields associated with flame propagation, and the flow ahead of the flame, were analyzed, with a particular focus on (though not limited to) the flame-generated compression waves and the time evolution of the radial flow velocity. The exponent $n$ in Eq. (4.1) has been reported in the range 4/3 $\leq n \leq 3 / 2$ [215-219]. Similar to work [182], in the present dissertation, $n=1.4$ was employed in the majority analysis, though the impact of varying $n$ has also been analyzed.

Demir et al. [182] combined the above analysis with the mechanisms of finger flame acceleration [184] into a unified formulation for a fire scenario in an unobstructed passage, with the evolution of the flame "skirt" position $R_{f}(t)$, the flame tip position $X_{t i p, f}(t)$, and the flame tip velocity $U_{t i p, f}(t)$ given by [182]:

$$
\begin{aligned}
& R_{f}(t)=\frac{\Theta H}{\Theta-1}\left\{1-\exp \left[-\frac{\Theta-1}{\Theta H}\left(k_{D L}^{n-1}\left(\frac{\Theta U_{f}}{n}\right)^{n}\right) t^{n}\right]\right\}, \\
& X_{t i p, f}=\frac{\Theta H}{\Theta-1}\left\{\exp \left[\frac{\Theta-1}{\Theta} \frac{k_{D L}^{n-1}}{H}\left(\frac{\Theta U_{f}}{n}\right)^{n} t^{n}\right]-1\right\},
\end{aligned}
$$




$$
\frac{d X_{t i p, f}}{d t}=U_{t i p, f}=n k_{D L}^{n-1}\left(\frac{\Theta U_{f}}{n}\right)^{n} t^{n-1} \exp \left[\frac{\Theta-1}{\Theta} \frac{k_{D L}^{n-1}}{H}\left(\frac{\Theta U_{f}}{n}\right)^{n} t^{n}\right] .
$$

The readers are referred to [182] for details of the formulation. Similar to [182], the thermal chemical parameters of the combustible mixture were taken as tabulated in [220]. In unobstructed passages, the formulation (4.2) - (4.4) will work until the instant when the flame skirt touches an obstacle, $t_{o b s}$, which is calculated from the condition $R_{f}\left(t_{o b s}\right)=(1-\alpha) H$ in Eq. (4.2) as

$$
\begin{gathered}
t_{o b s}=\frac{n}{\Theta U_{f}}\left\{\frac{\Theta H}{(\Theta-1) k_{D L}^{n-1}} \ln \left[\frac{\Theta}{1+\alpha(\Theta-1)}\right]\right\}^{1 / n}, \\
X_{t i p, f}\left(t_{o b s}\right)=\frac{\Theta(1-\alpha) H}{1+\alpha(\Theta-1)},
\end{gathered}
$$

where $X_{t i p, f}\left(t_{o b s}\right)$ is the corresponding flame tip position found from Eq. (4.3). Similarly, the respective flame tip velocity, $U_{t i p, f}\left(t_{o b s}\right)$, can be found from Eq. (4.4).

The formulation (4.2) - (4.4) does not work for $t>t_{o b s}$ because the obstacles come to play in this case. Consequently, in order to extend the coalmining fire scenario beyond $t_{o b s}$, the obstacles should be accounted in the formulation. In this respect, here we briefly summarize the Bychkov mechanism of fast flame acceleration in obstructed channels [206]. Theoretically, a limit of tightlyplaced obstacles, $\Delta z \ll \alpha H$, was adopted, which allows treating the flow between the obstacles as laminar. It is nevertheless noted that the Bychkov model [206] is applicable even far beyond the restriction $\Delta z \ll \alpha H$; namely, it is valid as long as $\Delta z \leq \alpha H$ or even $\Delta z \leq H$ as validated in the computational study [178]. Thus, it should be enough to keep the obstacles spacing less than the obstacle height, thereby having their ratio not exceeding unity, $\Delta z / \alpha H<1$ (though the tighterthe better, of course). In this respect, the assumption of a laminar flow between the obstacles might be waved, up to some extent. For instance, the Eulerian assumption of a non-viscous flow might lead to a similar $x$-direction velocity profile without imposing tightly placed obstacles. However, in that case, there would be additional effects of a vertical motion between the obstacles, presumably, leading to pulsations, which would need to be studied. Therefore, as of now, the flow was assumed to be laminar in the pockets between the obstacles.

With such a laminar flow approach, the flame front inside the pockets may be taken as locally planar all the time, thus spreading in the $x$-direction with the instantaneous global flame velocity 
$U_{D L}(t)$. As the burnt matter expands with a thermal expansion ratio $\Theta$, the flow is pushed out of the "pockets". Coming into a central part of the passage, the flow changes its direction and pushes the flame forward in the $z$-direction towards the exit. This creates a positive feedback between the flame and the pockets as the flame is pushed forward, thereby creating new pockets behind it.

It is emphasized that the flame dynamics and morphology are controlled by the processes occurring in the obstructed segment of the passage - not in its unobstructed, central part. Indeed, even though the flame tip is driven by a powerful jet-flow; such a jet-flow is generated due to delayed burning in the pockets between the obstacles. Moreover, the total burning rate (which grows extremely fast) correlates well with the total surface area of the flame front, which is mainly provided by the flame segments in the pockets between the obstacles. Consequently, the shape (surface area) of the flame tip does not play a seemed role in the flame surface area and, therefore, the total burning rate (even if the tip would be very corrugated, this would not change the total flame surface area and the total burning rate). For this reason, the shape of the flame tip can be assumed planar all the time [206, 207]. With this assumption, the flow in a free part of the passage is potential.

With methane-air and propane-air laminar burning velocities not exceeding $U_{f}=0.3 \sim 0.4 \mathrm{~m} / \mathrm{s}$ [220] and the respective speeds of sound circa $c_{0} \sim 350 \mathrm{~m} / \mathrm{s}$, the initial Mach number associated with flame propagation appears very small, $M a \equiv U_{f} / c_{0} \sim 10^{-3} \ll 1$. This enables to employ a low Mach-number model of an incompressible flow, $M a \rightarrow 0$, similar to Ref. [182]. This model has been widely employed analytically and verified by fully-compressible computational simulations in previous works $[180,207]$. The model has been justified to work well at the initial, essentially subsonic stage of flame acceleration, and to provide reasonable evaluations even in the case of compressible flows. It is nevertheless recognized that the model will break when the burning velocity will approach near-sonic values. Such a limitation of the model and the way to overcome it will be commented in Sec. 4.3.3. For a 2D geometry, the incompressible continuity equation reads

$$
\partial U / d x+\partial V / d y=0,
$$

Similar to the Bychkov model [206, 207], here we employ the assumption of a potential flow in the burnt gas, which was validated by the computational simulations in Ref. [207]. It is nevertheless noted that, in a real passage, obstacles will generate vorticity at least in the central region close to the obstacles. At the same time, according to Ref. [178], though the flow in the 
burnt gas is generally rotational in practice, because of the curved flame shape, it can be treated as potential close to the wall when the flame front is locally planar, which is enough to certify the Bychkov approach [178].

The question about vorticity and rotational region has been addressed, in part, in Refs. [178,221]. According to the simulations [221], vorticity provides a notable impact on the Bychkov mechanism only if the obstacles spacing exceeds the passage radius, $\Delta Z>R$, with the Bychkov model being fully broken if $\Delta Z=2 R$. Obviously, such $\Delta Z$ is much larger than what usually means tightlyplaced obstacles, $\Delta Z \leq \alpha R$, say $\Delta Z / R=0.2 \sim 0.6$ [178], thereby justifying the potential flow approach. As a result, considering the potential flow in the free part of the passage, with the boundary condition

$$
V=-(\Theta-1) U_{f} \text { at } y=(1-\alpha) H,
$$

we find the flow velocity and the flame propagation speed with respect to the burnt matter:

$$
\begin{gathered}
(U ; V)=\frac{(\Theta-1) U_{f}}{(1-\alpha) H}(x,-y), \\
\frac{d X_{t i p, o}}{d t}=\frac{(\Theta-1) U_{f}}{(1-\alpha) H} X_{t i p, o}+\Theta U_{f},
\end{gathered}
$$

which mathematically expresses the fact that the flame tip velocity in the laboratory reference frame equals the velocity of the burnt matter flow, $(\Theta-1) U_{f} X_{t i p, o} /(1-\alpha) H$, taken at the flame tip location, plus the flame tip velocity with respect to this flow, $\Theta U_{f}$. While both terms in the right-hand-side (RHS) of Eq. (4.10) are, initially, of the same order, the first ("flow") term will dominate over the second ("flame") term soon because the value $X_{t i p, o}(t)$ grows promptly with time. Equation (4.10) summarizes the original formulation [206], which yields exponential acceleration,

$$
X_{\text {tip.o }} \propto \exp (\sigma t), \text { with } \sigma=(\Theta-1) U_{f} /(1-\alpha) H .
$$

This formulation [206] is Reynolds-independent (scale-invariant), with $U_{f}=$ const. It is next revisited accounting for the DL instability. Specifically, we consider $U_{D L}(t)$ growing according to Eq. (4.1) until $t=t_{o b s}$ such that

$$
U_{D L}\left(t_{o b s}\right)=U_{f}\left\{\frac{\Theta H}{(\Theta-1) k_{D L}} \ln \left[\frac{\Theta}{1+\alpha(\Theta-1)}\right]\right\}^{(n-1) / n} .
$$


Thereafter, we assume that $U_{D L}$ remains at a saturated level of Eq. (4.12), $U_{D L \mid o b s}=U_{D L}\left(t_{o b s}\right)$, because a characteristic flame radius stops growing at this point. Then substituting $U_{D L \mid o b s}$ in Eq. (4.10) yields

$$
\frac{d X_{t i p, o}}{d t}=\frac{(\Theta-1) U_{D L \mid b b s}}{(1-\alpha) H} X_{t i p, o}+\Theta U_{D L \mid b b s}, \quad t>t_{o b s},
$$

as the evolution equation for the flame tip propagating through an array of obstacles, for $t>t_{o b s}$ (to inherit the evolution equation (4.10) valid for $t \leq t_{o b s}$ ). Integrating Eq. (4.13) with a matching condition $\left.X_{t i p, o}\right|_{t=t_{o b s}}=X_{t i p, f}\left(t_{o b s}\right)$ of Eq. (4.6) yields the solution for $X_{t i p, o}$ and $U_{t i p, o}$ in a form

$$
\begin{aligned}
& X_{t i p}=\frac{\Theta(1-\alpha) H}{1+\alpha(\Theta-1)}\left\{2 \exp \left[\frac{(\Theta-1)}{(1-\alpha)} \frac{U_{D L}\left(t_{o b s}\right)}{H}\left(t-t_{o b s}\right)\right]-1\right\}, \\
& \frac{d X_{t i p}}{d t}=U_{t i p}=2 \frac{\Theta(\Theta-1) U_{D L}\left(t_{o b s}\right)}{1+\alpha(\Theta-1)} \exp \left[\frac{(\Theta-1)}{(1-\alpha)} \frac{U_{D L}\left(t_{o b s}\right)}{H}\left(t-t_{o b s}\right)\right] .
\end{aligned}
$$

The flame run-up distance, which is conventionally defined as the distance at which the flame velocity reaches the speed of sound, $c_{0}$, is also determined. Namely, Eq. (4.15) gives the run-up time,

$$
t_{r u d}=t_{o b s}+\frac{(1-\alpha)}{(\Theta-1)} \frac{H}{U_{D L}\left(t_{o b s}\right)} \ln \left[\frac{c_{0}(1+\alpha(\Theta-1))}{2 \Theta(\Theta-1) U_{D L}\left(t_{o b s}\right)}\right],
$$

and substituting $t_{r u d}$ into Eq. (4.14) gives the corresponding flame run-up distance, $X_{\text {rud }}$.

It is recognized that a number of effects remained beyond consideration in the present work. For instance, only the DL flame instability is accounted as the agent triggering unsteadiness, without considering turbulence or other combustion instability modes such as the diffusional-thermal (DT), Rayleigh-Taylor (RT), or Kevin-Helmholtz (KH) instabilities. In the future, one can potentially incorporate these features into the analysis by replacing the quantities $\lambda_{D L}$ and $U_{D L}(t)$, Eq. (4.1) by the respective DT/RT/KH/turbulence-induced parameters. In addition, analysis of partly-open obstructed duct, as in the pioneering Taylor-Bimson (TB) model [222], would be of interest.

The analysis is next extended to a gaseous-dusty environment by using a modified version of the Seshadri formulation $[182,202]$ that expresses the laminar flame speed as a function of the local thermal-chemical properties of the gas and dust particles (inert; such as sand, combustible; i.e. coal, and combined) in the form [182] 


$$
U_{d, f}=U_{f} \sqrt{\frac{\phi_{s}}{\phi}} \sqrt{\frac{C_{P}}{C_{T}}}\left(\frac{T_{f}}{T_{b}}\right)^{2}\left(\frac{T_{b}-T_{u}}{T_{f}-T_{u}}\right) \sqrt{\exp \left(\frac{E\left(T_{f}-T_{b}\right)}{T_{f} T_{b} R_{u}}\right)},
$$

where $\phi_{s}$ is the effective equivalence ratio of the dusty-gaseous-air mixture in the presence of particles,

$$
\phi_{s}=\frac{\left[\left(\left(m_{\text {fuel }}^{m}\right) /\left(M_{C H_{4}}\right)\right) /\left(\left(m_{\text {air }}^{m}\right) /\left(M_{\text {air }}\right)\right)\right]_{\text {act }}}{\left[\left(\left(m_{C H_{4}}^{m}\right) /\left(M_{C H_{4}}\right)\right) /\left(\left(m_{\text {air }}^{m}\right) /\left(M_{\text {air }}\right)\right)\right]_{s t}}
$$

$M_{C H 4}, M_{\text {air }}$ are the respective molar masses; $m_{C H 4}^{m}, m_{\text {air }}^{m}$ and $m_{f u e l}^{m}$ are the original masses per unit volume for a given equivalence ratio; $C_{T}=C_{P}+C_{S} n_{S} V_{s} \rho_{s} / \rho$ is the whole specific heat of the mixture, containing the components for the gas, $C_{P}$, and dust particles, $C_{s} ; \rho_{s}$ is the density of a single dust particle while $\rho=\rho_{u}+c_{s}$ is that for the gaseous-dusty fuel-air mixture, with the density of the gas $\rho_{u}$ and concentration of the dust particles $c_{s} ; n_{s}=\left(c_{s} / \rho_{s}\right) / V_{s}$ is the number of particles per unit volume, with $V_{s}=4 \pi r_{s}^{3} / 3$ being the volume of a single particle and $r_{s}$ the particle radius; $R_{u}=8.314 \mathrm{~J} /(\mathrm{mol} \cdot \mathrm{K})$ is the universal gas constant; $T_{u}=300 \mathrm{~K}$ is the reactants temperature and $T_{b}$ is the adiabatic flame temperature based on the purely methane-air equivalence ratio. Similar to Ref. [185], here $T_{b}$ is calculated as a $5^{\text {th }}$-order polynomial function of the equivalence ratio $\phi[223]$

$$
\begin{aligned}
& T_{b}=\left(-2.21 \times 10^{4}\right) \phi^{5}+\left(8.042 \times 10^{4}\right) \phi^{4}+\left(-1.171 \times 10^{5}\right) \phi^{3} \\
& +\left(8.471 \times 10^{4}\right) \phi^{2}+\left(-2.854 \times 10^{4}\right) \phi+4.89 \times 10^{3}
\end{aligned}
$$

valid in the range $0.6 \leq \phi \leq 1.6$ [223]. Knowing $\phi_{s}$ from Eq. (4.18) and calculating a new flame temperature for the dusty-gaseous mixture, $T_{f}^{*}$, one can find $U_{d, f}$, Eq. (4.17), to be employed in the formulation in the previous section.

Next, the methodology of Refs. [182, 203] was employed. Unlike a combustible dust, an inert particle acts as a heat sink because it absorbs some heat from the flame and reduces the flame temperature. For $\phi \leq 1$, methane-air combustion, the global chemical reaction is described by the equation

$$
\phi \mathrm{CH}_{4}+2\left(\mathrm{O}_{2}+3.76 \mathrm{~N}_{2}\right) \Rightarrow \phi C \mathrm{O}_{2}+2 \phi \mathrm{H}_{2} \mathrm{O}+7.52 \mathrm{~N}_{2}+4(1-\phi) \mathrm{O}_{2}
$$


such that the heat release in the process of burning of $\phi$ moles of $\mathrm{CH}_{4}$ and $2 \times(1+3.76)=9.52$ moles of air is given by [182,203]

$$
Q_{\phi}=\left(T_{b}-T_{u}\right) \sum C_{p} n_{\text {product }}
$$

where $n_{\text {product }}$ is the number of moles of the burning products, which depends on the equivalence ratio $\phi$. Assuming that the entire heat released from the reaction is used to raise the temperature of the mixture, Eq. (4.21) can be modified into an expression for the volumetric heat release from $\mathrm{CH}_{4}$-air combustion of a given $\phi$ as follows [182]

$$
Q=\left[\left(T_{b}-T_{u}\right) \sum C_{P} . n_{\text {product }}\right] \frac{n_{\text {air }}}{9.52\left(V_{C H_{4}}+V_{\text {air }}\right)} .
$$

It is next assumed that a flame with particles releases the same amount of heat while it is also influenced by the temperature rise of particles [203]. Then Eq. (4.22) can be extended as [182]

$$
Q=\left[\left(T_{f}^{* *}-T_{u}\right) \sum C_{P} . n_{\text {product }}\right] \frac{n_{\text {air }}}{9.52\left(V_{C_{4}}+V_{\text {air }}\right)}+c_{s} C_{s}\left(T_{f}^{* *}+T_{u}\right)+L_{v},
$$

where $L_{v}=0.01 w_{v} \Delta h_{C H 4}$ [203] is the heat of gasification per unit volume. From the last equation, the secondly-revised flame temperature, $T_{f}^{* *}$, is calculated as

$$
T_{f}^{* *}=\frac{Q-L_{v}}{\frac{n_{\text {air }}}{9.52\left(V_{\mathrm{CH}_{4}}+V_{\text {air }}\right)} \sum C_{p} n_{\text {product }}+c_{s} C_{s}}+T_{u} .
$$

Finally, the effect of combination of the inert and combustible dust particles are accounted by averaging the adiabatic flame temperature over those values associated with both effects separately, $T_{f}^{* * *}=\left(T_{f}^{*}+T_{f}^{* *}\right) / 2$. Similarly to a combustible dust case, $T_{f}^{* *}$ and $T_{f}^{* * *}$ are used in Eq. (4.17) to find a new laminar flame speed $U_{d, f}$ for the inert and combined dust particle incorporations, respectively. As of now, all the particles are assumed to be distributed uniformly inside a coalmining passage; non-uniform dust distributions can be considered elsewhere, for instance, following the method of Ref. [224].

It is interesting what would happen to the DL cutoff wavelength $\lambda_{D L}$ when the environment is extended from the gaseous to the gaseous-dusty environment. To be self-consistent with the Seshadri formulation (which actually imitates a multi-phase system by an "effective" fluid with modified properties, adjusted due to the presence of solid particles), here $\lambda_{D L}$ is considered as the 
quantity devoted to such an effective fluid. Consequently, $\lambda_{D L}$ will be given by the same formula as used here, though the variables in this formula $\left(U_{f}, L_{f}\right)$ will be adjusted due to the presence of dust particles.

Another justification for this approach can also be provided. Namely, because $\lambda_{D L} \propto L_{f}$, if the flame thickness does not change much when the dust particles are laden, then the value $\lambda_{D L}$ as well as the onset and emergence of the DL instability would not change much too. It is nevertheless recognized that a rigorous analysis of the DL instability of gaseous-dusty flames will require a further study, with extra features to be incorporated. In particular, the transport processes such as heat transfer can be modified in an intriguing way - in particular, due to radiation.

\subsubsection{Results and Discussion in a 2D Geometry}

Here, the essence of an intensive parametric study performed is presented and discussed. In the analysis, variable thermal-chemical parameters (i.e. $\Theta$ and $U_{f}$ ) of a gaseous methane-air mixture (with no dust) were taken as the functions of equivalence ratio $\phi$ as tabulated in [220]. Figure 56 presents the flame tip position, $X_{t i p}$, Fig. 56a, and its velocity, $U_{t i p}$, Fig. 56b, versus time for various blockage ratios such as $\alpha=0,1 / 3,1 / 2,2 / 3$. The first case, $\alpha=0$, was that with no obstacles, reproducing, completely, the situation of "finger + DL" flame acceleration of [182]. It was recalled one more time that this acceleration was limited in time such that the flame would start decelerating when its "skirt" touches a sidewall at $t \sim 0.089 \mathrm{~s}$. In contrast, in obstructed channels, $\alpha>0$, the flame tip position and velocity deviated from the finger flame acceleration scenario at $t_{o b s}$, and this led to faster flame acceleration until the DDT event occurred. It should be recalled, at this point, that approaching the near-sonic values by the flame front will eventually break the incompressible approach, adopted in Eq. (4.7), and the entire present formulation. Indeed, to describe the DDT stage accurately, the impacts of gas compressibility must be incorporated into the present analysis. This can be done by considering the formulation of Sec. 4.3.2 as the zeroth-order approach in $M a \rightarrow 0$, and then extend it to account for the finite $M a$ according to the methodology, employed earlier for unobstructed [183] and obstructed [225]

geometries. Such a rigorous extension of the present formulation to account for the compressibility effects is presented in Sec. 4.3.6 [226]. It was also noticed that the analytical incompressible formulation of Sec. 4.3.2 did not involve pressure as a parameter (except for the fact that pressure 
came indirectly through the thermal-chemical parameters such as $U_{f}$ or $\Theta$, which were taken for ambient, atmospheric pressure indeed in the present section). However, if the present formulation is extended to account for a finite $M a$, as discussed above, then pressure, its variations and gradient, will be directly involved in a revised formulation, with the ambient, atmospheric pressure imposed as the initial conditions and a boundary condition in the open side of the passage.

Overall, acceleration observed in Fig. 56 even exceeded that of Bychkov et al. [206] by orders of magnitude, thereby certifying a significant impact of the DL instability on the flame/fire evolution in an obstructed coalmining passage. It should be mentioned that Fig. 56 as well as the following Figs. 58-63 employed the exponent $n$ of Eq. (4.1) to be the same as that in Ref. [182], i.e. $n=1.4$. This was in order to compare the present work with the "unobstructed burning accident" theories [182] (as well as with the original Bychkov theories [206, 207], which did not consider the DL instability, thereby having $n=1$ by default). It is noted, in this respect, that an appropriate choice of the factor $n$ is not finalized yet. Indeed, while various experimental studies such as Gostintsev et al. [215], Bradley et al. [216], Molkov et al. [217], Kim et al. [218] reported $n \approx 1.5$, the Princeton experiments in a dual chamber at elevated pressures suggested $n=1.3 \sim 1.33$; see Jomaas et al. [219] and numerous references therein. The theoretical work by Akkerman \& Law [227] has provided an attempt to explain such a discrepancy between various experiments by the flame-acoustic coupling, which modifies $n$. Moreover, the quantity of $n$ can also be modified by the RT and DT instabilities and turbulence (assuming that the general trend of $R_{f}(t)$ being timedependent power law remains).

$n$ in this range $4 / 3 \leq n \leq 3 / 2$ was nevertheless considered and its impact on the present formulation was scrutinized. Figure 57 compares the time evolutions of the flame tip positions and velocities for stoichiometric methane-air burning considering $n=1.33,1.4$, and 1.5 in 2D geometry. It is seen that the variations of $n$ impacted the flame position, velocity and acceleration substantially, though the effect was quantitative but not qualitative. As excepted, flame acceleration in the case of $n=1.5$ proceeded noticeably faster than for $n=1.4$, whereas a flame with $n=1.33$ accelerated noticeably slower as compared to that with $n=1.4$ (provided that other combustion characteristics were kept the same). Anyway, without the final answer to the question about an appropriate choice for $n$, in the rest of this dissertation a median value, $n=1.4$, was 
used, the same as that in the unobstructed theories [182]. This allowed to compare with Ref. [182], thereby separating the impact of obstacles.
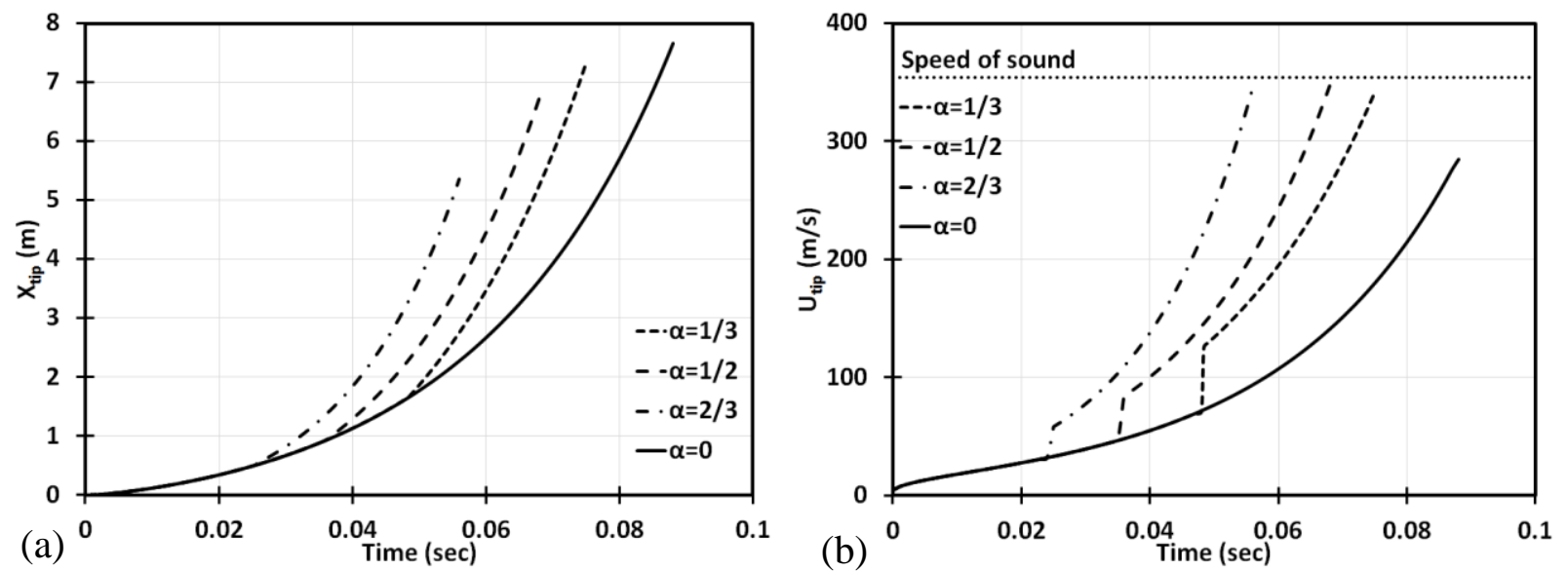

Figure 56: Time evolution of the flame tip position $X_{\text {tip }}$ (a) and velocity $U_{\text {tip }}$ (b) for the stoichiometric $(\phi=1)$ methane-air mixture with various blockage ratios $\alpha=0,1 / 3,1 / 2,2 / 3(n=1.4)$.
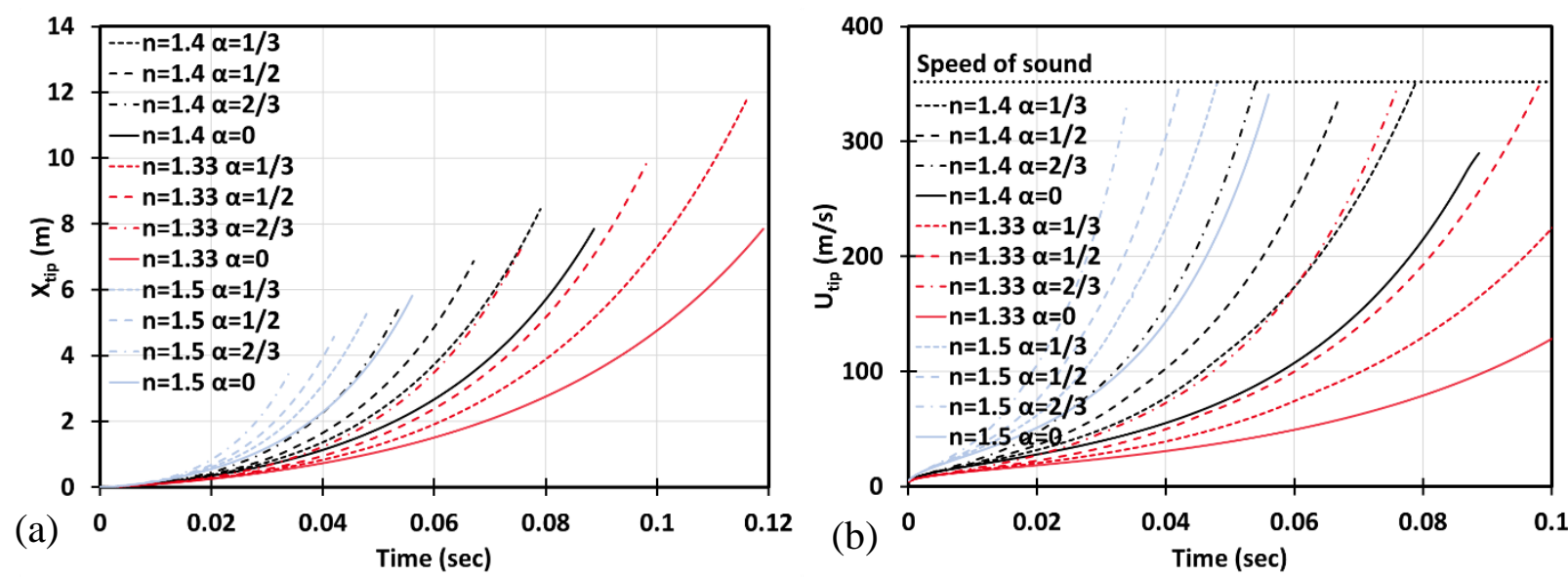

Figure 57: Time evolution of the flame tip position $X_{\text {tip }}$ (a) and velocity $U_{\text {tip }}$ (b) in $2 \mathrm{D}$ geometry for stoichiometric $\mathrm{CH}_{4}$-air burning with various blockage ratios $\alpha=0,1 / 3,1 / 2,2 / 3$ and various power factors $n=1.33,1.4,1.5$.

Next, stoichiometric $(\phi=1)$ gaseous methane-air combustion considered in Fig. 56 was extended to the equivalence ratios in the range $0.6 \leq \phi \leq 1.4$; see Figs. 58 and 59. Figures $58(\mathrm{a}, \mathrm{b})$ presents for the variety of equivalence ratios such as $\phi=0.8,1.0,1,2$ for $\alpha=0,1 / 3,1 / 2,2 / 3$. It is seen that a fuel-lean flame with $\phi=0.8$ propagated/accelerated much slower than the $\phi \geq 1$ flames. This happened because of much lower $U_{f}$ (and thereby larger $L_{f}$ and smaller $k_{D L}$ ) occurring at such a fuel-lean condition. In contrast, near-stoichiometric flames appeared to accelerate very fast. In fact, a slightly fuel-rich flame of $\phi \sim 1.1$ (not shown in the figure) provided fastest acceleration. 
However, the further increase in $\phi$ will actually moderate flame acceleration as compared to the stoichiometric mixture. It is recalled again that in this theory, flame acceleration is unlimited in time so that even relatively slow flames may eventually trigger a detonation provided sufficiently long passage (and time). For instance, even for $\phi=0.7, \alpha=1 / 3$, Eq. (4.16) predicts the DDT at $t_{\text {rud }} \sim 0.38 \mathrm{~s}$, and this timing will drastically reduce with $\alpha$ and/or $\phi$.
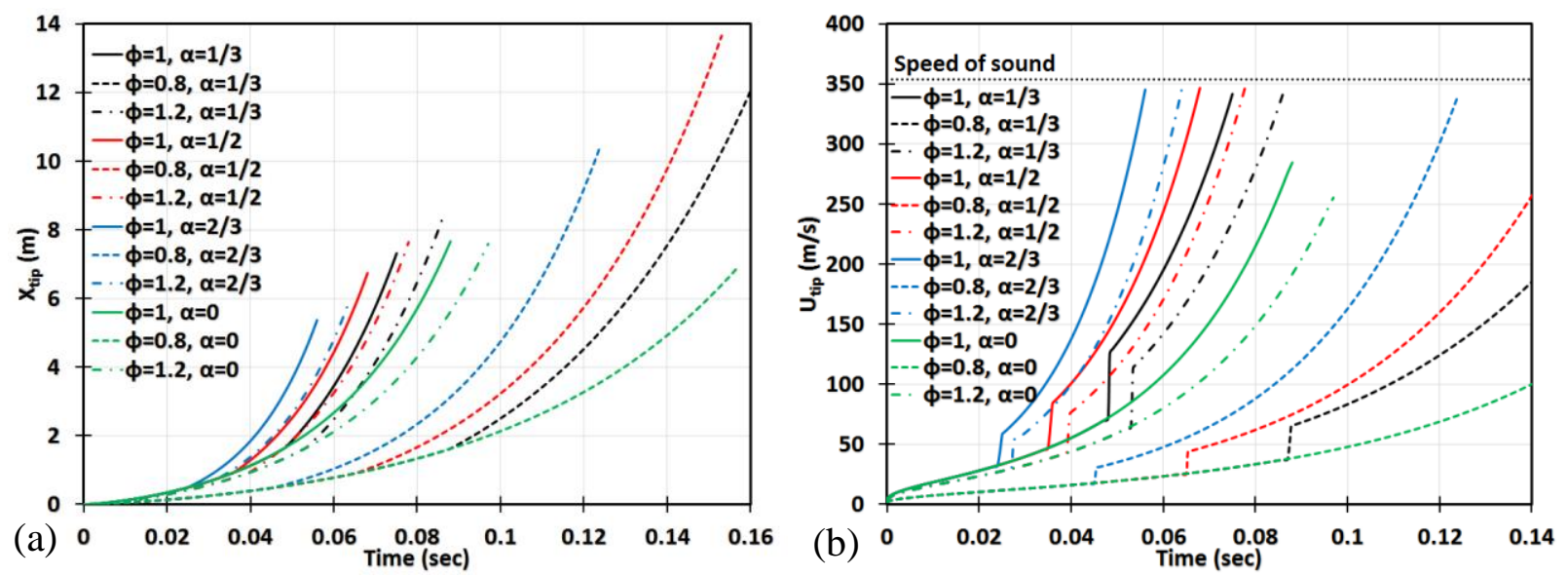

Figure 58: Time evolution of the flame tip position $X_{\text {tip }}$ (a) and velocity $U_{\text {tip }}$ (b) for the lean $(\phi=0.8)$, stoichiometric $(\phi=1)$ and rich $(\phi=1.2)$ methane-air mixtures with various blockage ratios: $\alpha=$ $0,1 / 3,1 / 2,2 / 3$.

The flame propagated slower if the equivalence ratio deviates further from stoichiometry, as depicted in Fig. 59, showing the evolutions of the position and velocity of the $\phi=0.6$ and $\phi=$ 1.4 methane-air flames (to avoid messy, the plots for various equivalence ratios between Figs. 58 and 59 were split). It is also seen that the duration of acceleration of the highly lean/rich flames in Fig. 59 exceeded that of the stoichiometric or slightly lean/rich flames in Fig. 58. In particular, without obstructions, the $\phi=0.6$ flame stopped accelerating after advancing $5.8 \mathrm{~m}$ and attaining the maximal velocity of $24.6 \mathrm{~m} / \mathrm{s}$ before the flame skirt contacted the wall. Obviously, this acceleration scenario does not end thereafter, if the obstacles are added to the passage wall. In contrast, the flame front will keep accelerating until its speed reaches the speed of sound and, eventually, the detonation is triggered. It is noted that the obstacles facilitated flame acceleration as compared to that in an unobstructed passage, and the impact of obstacles seemed to be more important for the $\phi=0.6$ flames than for the $\phi=1.4$ ones: the lines for $\phi=0.6$ with various $\alpha$ went more widely than those for $\phi=1.4$. It is also seen in Fig. 59 that the rich flames, $\phi=1.4$, accelerated faster than the lean flames, $\phi=0.6$; In the latter respect, the situation of highly non- 
stoichiometric combustion in Fig. 59 qualitatively resembled slightly non-stoichiometric burning in Fig. 58.
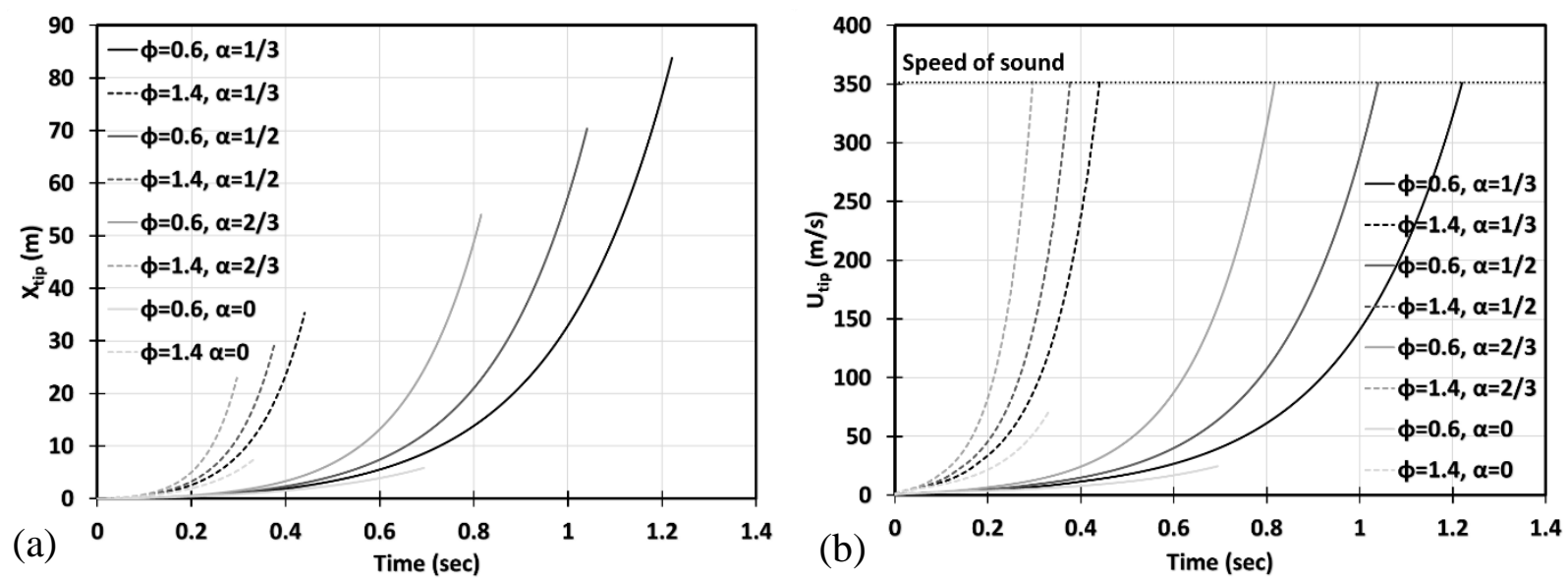

Figure 59: Time evolution of the flame tip position $X_{\text {tip }}$ (a) and velocity $U_{\text {tip }}$ (b) in a 2D geometry for highly-lean $(\phi=0.6)$ and highly-rich $(\phi=1.4) \mathrm{CH}_{4}$-air burning with various blockage ratios $\alpha=$ $0,1 / 3,1 / 2,2 / 3$.

Finally, the analysis was extended from a purely gaseous to a gaseous-dusty environment by using a modified Seshadri formulation for the laminar flame speed $U_{d, f}$ as a function of thermalchemical properties of the gas and dust particles as explained in the previous section. The combustible (e.g. coal) and inert (e.g. sand) particles as well as their combinations are considered.
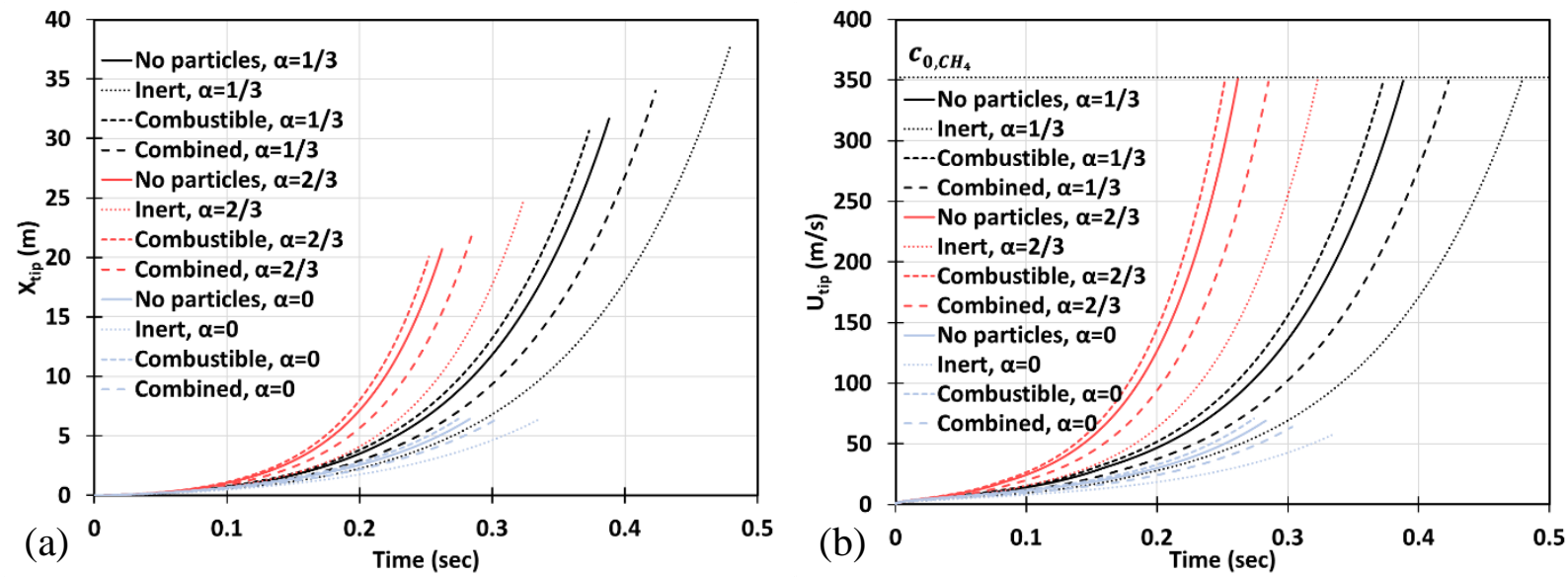

Figure 60: Time evolution of the flame tip position $X_{\text {tip }}$ (a) and velocity $U_{t i p}$ (b) in a $2 \mathrm{D}$ geometry for lean $\mathrm{CH}_{4}$-air burning of $\phi=0.7$, without and with dust particles (combustible, inert, and combined) of particle radius $\boldsymbol{r}_{\boldsymbol{s}}=\mathbf{7 5} \boldsymbol{\mu \mathrm { m }}$ and concentration $\boldsymbol{c}_{\boldsymbol{s}}=\mathbf{5 0} \mathrm{g} / \mathrm{m}^{3}$, for various blockage ratios: $\alpha=$ $0,1 / 3,2 / 3$.

As of now, the particles are assumed to be distributed uniformly inside the passage; non-uniform dust distribution requires a future work. Fig. 60 depicts the situation of gaseous-dusty combustion, 
with the dust of concentration $c_{s}=50 \mathrm{~g} / \mathrm{m}^{3}$ and of dust particles radius $r_{s}=75 \mu \mathrm{m}$ in the $2 \mathrm{D}$ geometry. The lean $(\phi=0.7)$ methane-air mixture and various blockage ratios $\alpha$ were employed, including the case of no obstacles, $\alpha=0$. It is seen that combined (combustible+inert) and inert dust moderated flame acceleration whereas combustible particles slightly facilitated flame propagation. The effect of obstacles was noticeable as well (compare with an unobstructed passage).

Next, the dust concentration was increased. Namely, in Fig. 61, it is promoted to $c_{s}=120 \mathrm{~g} / \mathrm{m}^{3}$ keeping the same particle radius, $r_{s}=75 \mu \mathrm{m}$, and other characteristic and geometry as in Fig. 60. It is seen that combustible dust promoted flame acceleration, while inert dust and its combination with combustible dust moderated the acceleration process for the particles of radius $r_{s}=75 \mu \mathrm{m}$.
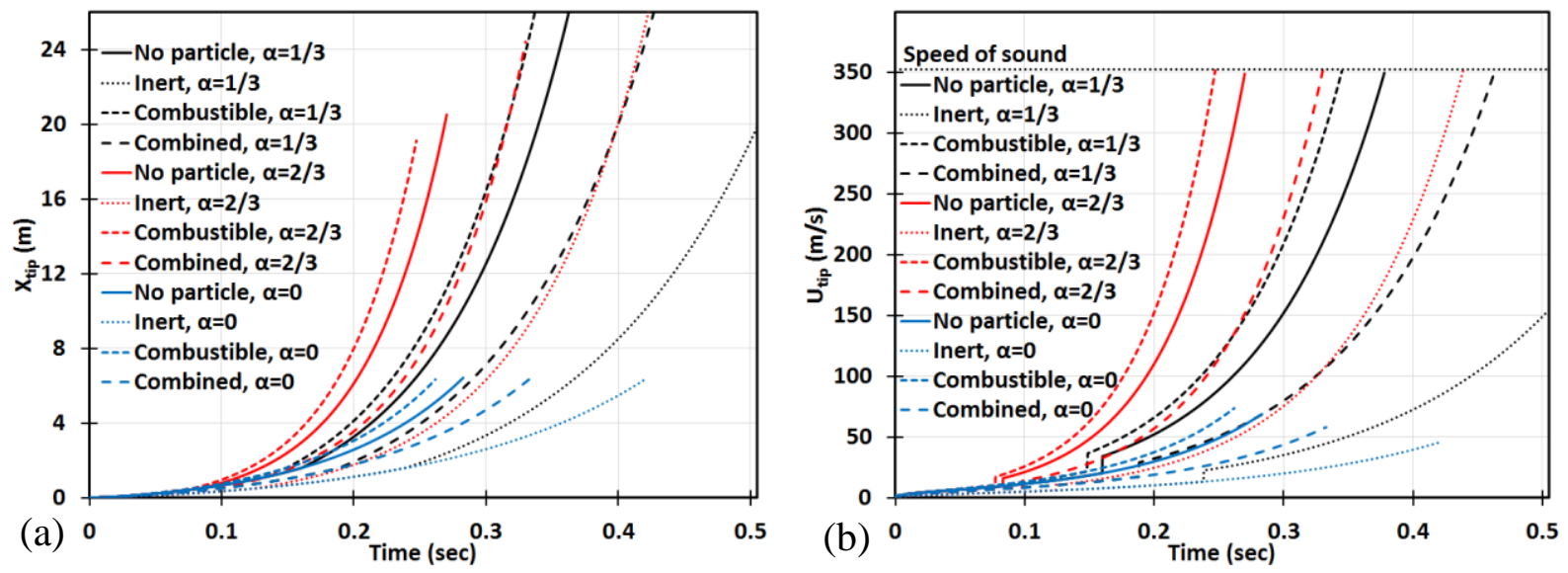

Figure 61: Time evolution of the flame tip $X_{\text {tip }}$ (a) and velocity $U_{\text {tip }}$ (b) for a lean methane air-mixture of $\phi=0.7$ with and without dust particles (inert, combustible, and combined) of radius $\boldsymbol{r}_{\boldsymbol{s}}=\mathbf{7 5} \boldsymbol{\mu m}$ and concentration $\boldsymbol{c}_{\boldsymbol{s}}=\mathbf{1 2 0} \mathbf{g} / \mathbf{m}^{3}$, for the blockage ratios: $\alpha=0,1 / 3,1 / 2,2 / 3$. 

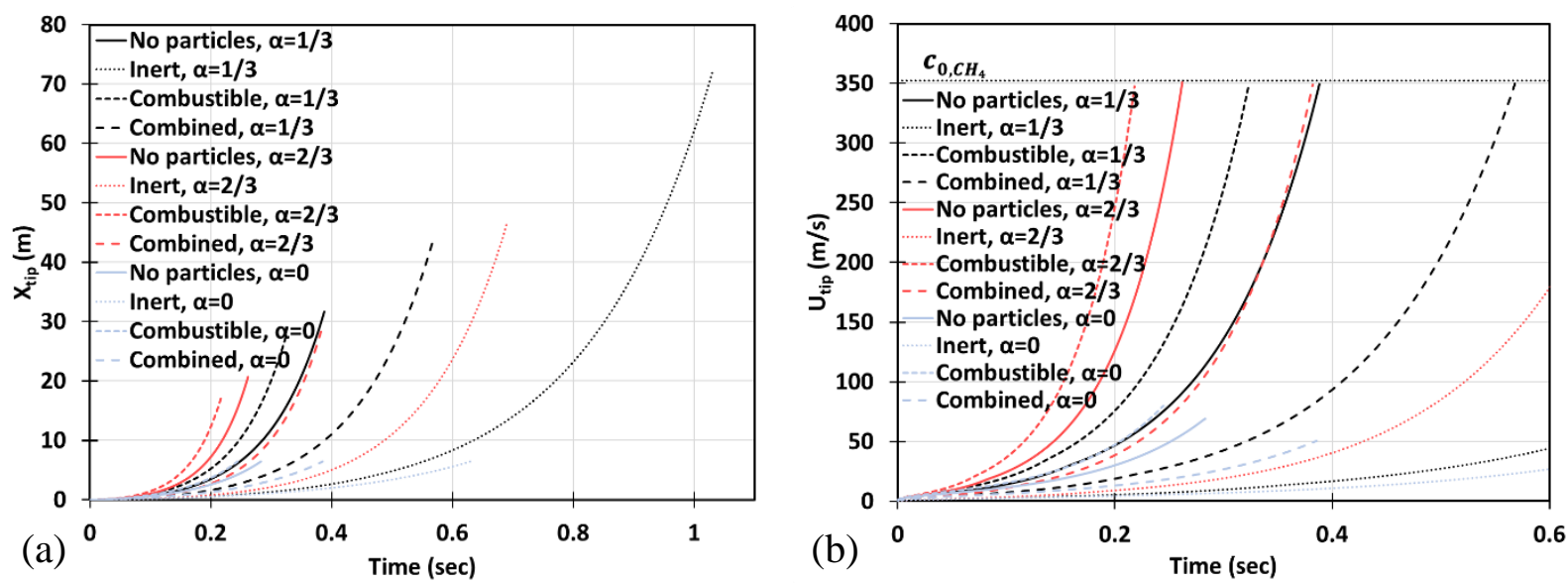

Figure 62: Time evolution of the flame tip position $X_{\text {tip }}$ (a) and velocity $U_{\text {tip }}$ (b) in a 2D geometry for lean $\mathrm{CH}_{4}$-air burning of $\phi=0.7$, without and with dust particles (combustible, inert, and combined) of

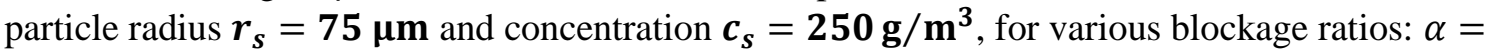
$0,1 / 3,2 / 3$.

The impact of blockage ratio is noticeable. Relatively high concentration has also been considered. Specifically, Fig. 62 presents the case of $c_{s}=250 \mathrm{~g} / \mathrm{m}^{3}$, with the particle size $r_{s}=75 \mu \mathrm{m}$, the same as in Figs. 60, 61. This investigation revealed that if keeping the dust particle size constant while increasing the number of particles (i.e. the concentration), the effect of particles became more important. This was observed from Figs. 60-62 that the flames in gaseous-dusty environments departed from the case of no dust particles more widely as the concentration increased. Similar to the cases of $c_{S}=50$ and $120 \mathrm{~g} / \mathrm{m}^{3}$, the combustible particles promoted flame acceleration whereas the combined (combustible+inert) and inert particles suppressed it in the case of $c_{S}=250 \mathrm{~g} / \mathrm{m}^{3}$ too.
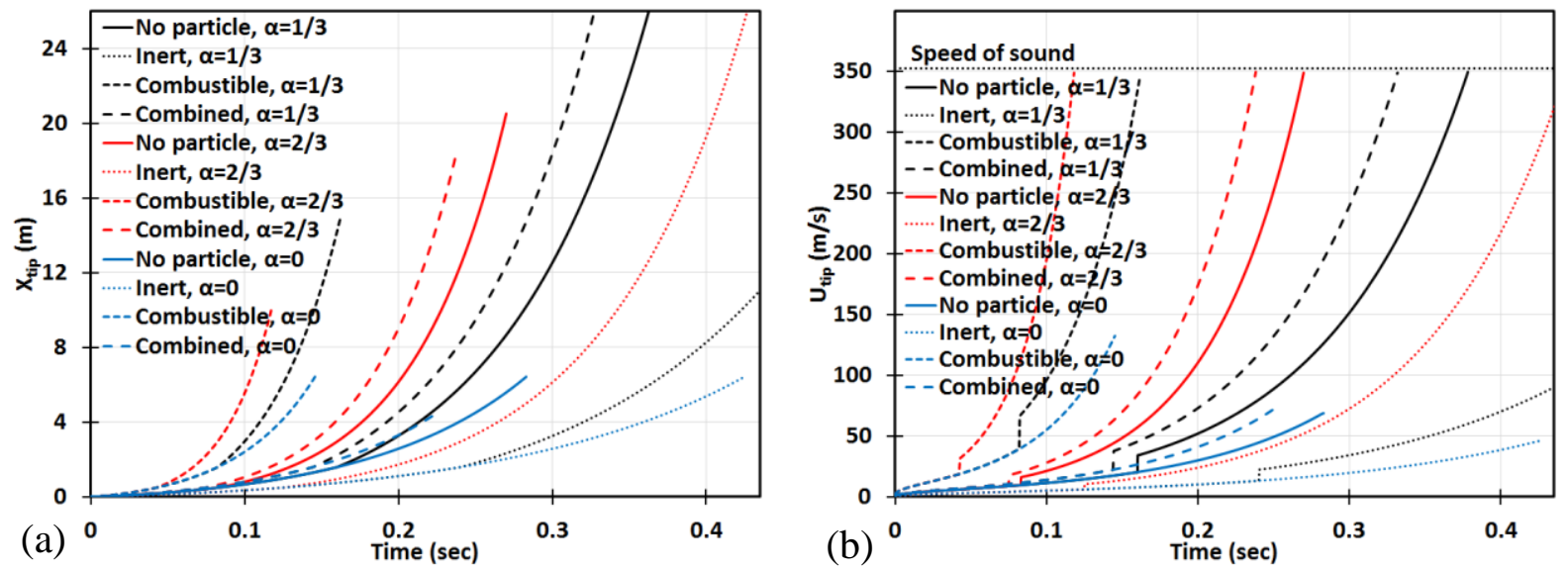

Figure 63: Time evolution of the flame tip $X_{\text {tip }}$ (a) and velocity $U_{\text {tip }}$ (b) for a lean methane air-mixture of $\phi=0.7$ with and without dust particles (inert, combustible, and combined) of radius $\boldsymbol{r}_{\boldsymbol{s}}=\mathbf{1 0} \boldsymbol{\mu m}$ and concentration $\boldsymbol{c}_{\boldsymbol{s}}=\mathbf{1 2 0} \mathbf{g} / \mathbf{m}^{3}$, for the blockage ratios: $\alpha=0,1 / 3,1 / 2,2 / 3$. 
Figures $63(\mathrm{a}, \mathrm{b})$ are the counterparts of Fig. $61(\mathrm{a}, \mathrm{b})$ for a smaller dust particle radius, $r_{s}=10 \mu \mathrm{m}$. It is seen from Fig. 63 that the smaller particles provided a stronger impact on flame propagation. Indeed, here the curve for the coal particles deviated noticeably larger from the case of no particles. It should be noted here that while the flame velocities did not exceed $35 \mathrm{~m} / \mathrm{s}$ for the particles of size $r_{s}=75 \mu \mathrm{m}$, in the case of $r_{s}=10 \mu \mathrm{m}$, the sound threshold of $352 \mathrm{~m} / \mathrm{s}$ for $\phi=0.7$ methaneair mixture was reached in the combustible coal gaseous-dusty environment during the time interval of approximately $0.118 \mathrm{~s}$. In contrast to a situation of $r_{s}=75 \mu \mathrm{m}$ in Fig. 61, the combined combustible-inert particles promoted flame acceleration in the case of $r_{s}=10 \mu \mathrm{m}$, Fig. 63. This shows that the heat sink effect of inert particles was dominated by the heat release effect of combustible particles when the size of the particles was smaller. As for the inert particles, similarly to the case of $r_{s}=75 \mu \mathrm{m}$, they suppressed flame acceleration for $r_{s}=10 \mu \mathrm{m}$ too. From the data (not shown here), it is seen that the aforementioned effects of all particles, i.e. combustible, inert, and combined, were promoted with $\alpha$ for-both particle sizes.

\subsubsection{Formulation for the Cylindrical Passage (Tube)}

Next, a cylindrical passage of radius $R=1.05 \mathrm{~m}$ was considered. The radius of a globallyspherical expanding flame obeys a power law, $R_{f} \propto t^{n}$, with $n=1.3 \sim 1.5$ [212] such that the instantaneous radial flame velocity with respect to the fuel mixture reads the same equations as Eq. (4.1). For a fire scenario in an unobstructed passage, the evolution of the flame "skirt" position $R_{f}(t)$, the flame tip position $Z_{t i p, f}(t)$, and the flame tip velocity $U_{t i p, f}(t)$ is updated as [182]:

$$
\begin{gathered}
R_{f}(t)=\frac{\Theta R}{\beta} \tanh \left(\frac{\beta}{\Theta R} k_{D L}^{n-1}\left(\frac{\Theta U_{f}}{n}\right)^{n} t^{n}\right), \\
Z_{t i p, f}=\frac{R}{2} \sqrt{\frac{\Theta-1}{\Theta}} \sinh \left(2 \sqrt{\frac{\Theta-1}{\Theta}} \frac{k_{D L}^{n-1}}{R}\left(\frac{\Theta U_{f}}{n}\right)^{n} t^{n}\right), \\
\frac{d Z_{t i p, f}}{d t}=U_{t i p, f}=\left(\Theta U_{f}\right)^{n}\left(\frac{k_{D L} t}{n}\right)^{n-1} \cosh \left(2 \sqrt{\frac{\Theta-1}{\Theta}} \frac{k_{D L}^{n-1}}{R}\left(\frac{\Theta U_{f}}{n}\right)^{n} t^{n}\right),
\end{gathered}
$$

where $\beta=\sqrt{\Theta(\Theta-1)}$; see [182] for details of the formulation. Similar to the 2D case, $n=1.4$ and the thermal-chemical parameters of the combustible mixture were taken as tabulated in Ref. [220]. In an unobstructed passage, the formulation (4.25) - (4.27) will work until the instant when the flame skirt touches an obstacle, $t_{o b s}$, which can be found from a condition $R_{f}\left(t_{o b s}\right)=(1-\alpha) R$ : 


$$
\begin{gathered}
t_{o b s}=\frac{n}{\Theta U_{f}}\left\{\frac{\Theta R}{2 \beta k_{D L}^{n-1}} \ln \left[\frac{\Theta+(1-\alpha) \beta}{\Theta-(1-\alpha) \beta}\right]\right\}^{1 / n}, \\
Z_{t i p, f}\left(t_{o b s}\right)=\frac{\Theta(1-\alpha) R}{\Theta-(\Theta-1)(1-\alpha)^{2}},
\end{gathered}
$$

where $t_{o b s}$ is found from Eq. (4.25), while Eq. (4.26) provides the corresponding flame tip position, $Z_{t i p, f}\left(t_{o b s}\right)$. Similarly, the respective flame tip velocity, $U_{t i p, f}\left(t_{o b s}\right)$, can be found from Eq. (4.27).

The formulation (4.25) - (4.27) does not work for $t>t_{o b s}$ because the obstacles come to play in this case. Along with the incompressible flow assumption, $\nabla \cdot \boldsymbol{u}=0$, and boundary condition at the border of the unobstructed part of the passage, $U_{r}=-(\Theta-1) U_{f}$ at $r=(1-\alpha) R$, the flow distribution and the flame evolution with respect to the burnt matter reads

$$
\begin{gathered}
\left(U_{z} ; U_{r}\right)=\frac{(\Theta-1) U_{f}}{(1-\alpha) R}(2 z ;-r), \\
\frac{d Z_{t i p, o}}{d t}=2 \frac{(\Theta-1) U_{f}}{(1-\alpha) R} Z_{t i p, o}+\Theta U_{f} .
\end{gathered}
$$

An additional increase in the acceleration rate occurs in the cylindrical-axisymmetric geometry, because the flame in the axisymmetric pockets expands with the radius as [207]

$$
R_{f, o}=(1-\alpha) R+U_{f}\left[t-t_{f}(z)\right]
$$

where $t_{f}(z)$ is the instant at which the fresh gas in a pocket between obstacles at the position $z$ starts burning (see Ref. [207] for the details) and $R_{f, o}$ represents the radial coordinate of flame skirt in an obstructed passage. Averaging the last term in Eq. (4.32) as

$$
\left\langle t-t_{f}(z)\right\rangle=(1-\alpha) R / 2 U_{f}(\Theta-1),
$$

yields a modified version of the evolution Eq. (4.31) for $Z_{t i p, o}$ as

$$
\frac{d Z_{t i p, o}}{d t}=2 \frac{(\Theta-1)}{(1-\alpha)} \frac{U_{f}}{R}\left(1+\frac{1}{2(\Theta-1)}\right) Z_{t i p, o}+\Theta U_{f}=\frac{(2 \Theta-1)}{(1-\alpha)} \frac{U_{f}}{R} Z_{t i p, o}+\Theta U_{f} .
$$

Equation (4.34) summarizes the original formulation [207], which yields exponential acceleration,

$$
Z_{\text {tip.o }} \propto \exp (\sigma t), \text { with } \sigma=(2 \Theta-1) U_{f} /(1-\alpha) R .
$$

This formulation is Reynolds-independent (scale-invariant), with $U_{f}=$ const. It is next revisited accounting for the DL instability. Specifically, $U_{D L}(t)$ will be considered growing according to Eq. (4.1) until $t=t_{o b s}$ such that 


$$
U_{D L}\left(t_{o b s}\right)=U_{f}\left\{\frac{\Theta R k_{D L}}{2 \beta} \ln \left[\frac{\Theta+(1-\alpha) \beta}{\Theta-(1-\alpha) \beta}\right]\right\}^{(n-1) / n} .
$$

Thereafter, $U_{D L}$ is assumed to remain at a saturated level of Eq. (4.36), $U_{D L \mid o b s}=U_{D L}\left(t_{o b s}\right)$, because a characteristic flame radius stops growing at this point. Then, substituting $U_{D L \mid o b s}$ in Eq. (4.34) yields

$$
\frac{d Z_{t i p, o}}{d t}=\frac{(2 \Theta-1)}{(1-\alpha)} \frac{U_{D L}\left(t_{o b s}\right)}{R} Z_{t i p, o}+\Theta U_{D L}\left(t_{o b s}\right), \quad t>t_{o b s}
$$

as the evolution equation for the flame tip propagating through an array of obstacles, for $t>t_{\text {obs }}$ (to inherit the evolution equation (4.31) valid for $t \leq t_{o b s}$ ). Integrating Eq. (4.37) with a matching condition $\left.Z_{t i p, o}\right|_{t=t_{o b s}}=Z_{t i p, f}\left(t_{o b s}\right)$ Eq. (4.34) yields the solution for $Z_{t i p, o}$ and $U_{t i p, o}$ in the form

$$
\begin{gathered}
Z_{t i p, o}=\frac{\Theta(1-\alpha) R}{(2 \Theta-1)}\left\{\left[\frac{2 \Theta-1}{\Theta-(\Theta-1)(1-\alpha)^{2}}+1\right] \exp \left[\frac{(2 \Theta-1)}{(1-\alpha)} \frac{U_{D L}\left(t_{o b s}\right)}{R}\left(t-t_{o b s}\right)\right]-1\right\}, \\
\frac{d Z_{t i p, 0}}{d t}=U_{t i p, o}=\Theta U_{D L}\left(t_{o b s}\right)\left\{\frac{2 \Theta-1}{\Theta-(\Theta-1)(1-\alpha)^{2}}+1\right\} \exp \left[\frac{(2 \Theta-1)}{(1-\alpha)} \frac{U_{D L}\left(t_{o b s}\right)}{R}\left(t-t_{o b s}\right)\right] .
\end{gathered}
$$

We also determine the flame run-up distance, which is conventionally defined as the distance at which the flame velocity reaches the sound speed. Namely, Eq. (4.39) gives the run-up time, $t_{\text {rud }}$,

$$
t_{r u d}=t_{o b s}+\frac{(1-\alpha)}{(2 \Theta-1)} \frac{R}{U_{D L}\left(t_{o b s}\right)} \ln \left[\frac{c_{0}}{\Theta U_{D L}\left(t_{o b s}\right)\left(\frac{2 \Theta-1}{\Theta-(\Theta-1)(1-\alpha)^{2}}+1\right)}\right],
$$

and substituting $t_{\text {rud }}$ of Eq. (4.40) into Eq. (4.38), the flame run-up distance, $Z_{\text {rud }}$, is found as

$$
Z_{\text {rud }}=\frac{\Theta(1-\alpha) R}{(2 \Theta-1)}\left\{\left[\frac{2 \Theta-1}{\Theta-(\Theta-1)(1-\alpha)^{2}}+1\right] \exp \left[\frac{(2 \Theta-1)}{(1-\alpha)} \frac{U_{D L}\left(t_{\text {obs }}\right)}{R}\left(t_{\text {rud }}-t_{\text {obs }}\right)\right]-1\right\} \text {. }
$$

This study was next extended to a gaseous-dusty environment by using Eqs. (4.17)-(4.24), similarly to 2D geometry analysis.

\subsubsection{Results and Discussion in a Cylindrical Geometry}

Here the results of an intensive parametric study performed were presented and discussed. Specifically, first, the homogeneously-gaseous methane-air mixture (with no dust) is considered, 
with the thermal-chemical parameters (i.e. $\Theta$ and $U_{f}$ ) being the functions of the equivalence ratio $\phi$ as tabulated in Ref. [220]. Figure 64 presents the evolution of the flame tip position $Z_{\text {tip }}$, Fig. 64a, and its velocity $U_{t i p}$, Fig. 64b, for the stoichiometric methane-air mixture, $\phi=1$, and various blockage ratios such as $\alpha=0,1 / 3,1 / 2,2 / 3$. The horizontal dotted line in Fig. 64b shows the speed of sound, being $\sim 354 \mathrm{~m} / \mathrm{s}$ for such a mixture. The curve with $\alpha=0$ describes the situation of no obstacles, and it reproduced, completely, the situation of "finger + DL" flame acceleration investigated by Demir et al. [182]. It is emphasized that although this acceleration was limited in time such that the flame front would start decelerating when its skirt contacted the sidewall at $t_{\text {wall }} \sim 0.0735 \mathrm{~s}$, in fact, according to prediction of the present formulation, this flame would overcome the sound threshold at $t_{r u d} \sim 0.0685 \mathrm{~s}<t_{\text {wall }}$, i.e. slightly prior deceleration. In the obstructed coalmining passages, $\alpha>0$, the flame accelerated according to the finger flame acceleration scenario, Eqs. (4.25) - (4.27), until the time $t_{o b s}$, when the flame skirt contacted an obstacle. Thereafter, for $t>t_{o b s}$, the obstacles came into play such that the flame tip position, $Z_{t i p, o}$, and its velocity, $U_{t i p, o}$, obeyed Eqs. (4.38) and (4.39), respectively, thereby deviating from the unobstructed case. These deviations led to faster flame velocity growth until the flame approached the speed of sound and triggered the DDT. It should be recalled, at this point, that approaching the near-sonic values by the flame front will eventually break the incompressible approach, adopted in Eq. (4.30), and the entire present formulation. Indeed, to describe the DDT stage accurately, the impacts of gas compressibility into the present analysis must be accounted. Overall, Fig. 64 shows that the obstacles influence a coalmine fire scenario significantly, making acceleration potentially unlimited, which was contrary to the case of no obstacles, which was considered in Ref. [182]. Besides, flame acceleration in Fig. 64 exceeded that from the original formulation [207] by orders of magnitude, thereby certifying that the DL instability facilitated obstacles-based acceleration. 

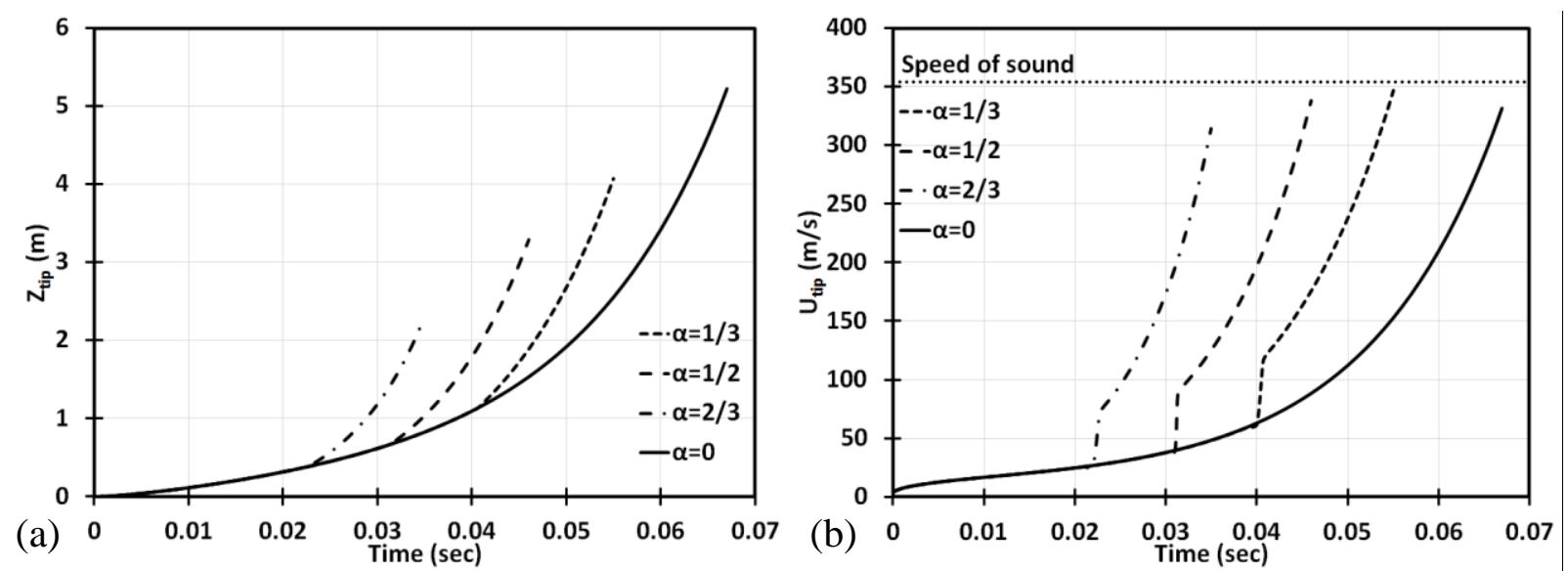

Figure 64: Evolution of the flame tip position $Z_{\text {tip }}$ (a) and velocity $U_{\text {tip }}$ (b) for the stoichiometric $(\phi=1)$ methane-air mixture with various blockage ratios $\alpha=0,1 / 3,1 / 2,2 / 3$.

Figs. 64 as well as the following Figs. 66-71 employ the exponent $n$ of Eq. (1) to be the same as that in Ref. [182], i.e. $n=1.4$. This is in order to compare the present work with the "unobstructed burning accident" theories [185] (as well as with the original Bychkov theories [206, 207], which did not consider the DL instability, thereby having $n=1$ by default). Moreover, $n$ in the range of $4 / 3 \leq n \leq 3 / 2$ was considered and its impact on the present formulation was scrutinized in cylindrical geometry. Specifically, Fig. 65 compares the time evolutions of the flame tip positions (a) and velocities (b) for stoichiometric methane-air burning considering $n=1.33,1.4$, and 1.5. It is seen that the variations of $n$ impacted the flame position, velocity and acceleration substantially, though the effect was quantitative but not qualitative. As excepted, flame acceleration in the case of $n=1.5$ proceeded noticeably faster than for $n=1.4$, whereas a flame with $n=1.33$ accelerated noticeably slower as compared to that with $n=1.4$ (of course, provided that other combustion characteristics are kept the same). Without a final answer to the question about an appropriate choice for $n$, in the rest of the analysis its median value, $n=1.4$, was used, the same as that in the unobstructed theories [182]. This allowed to compare with Ref. [182], separating the obstacles. 

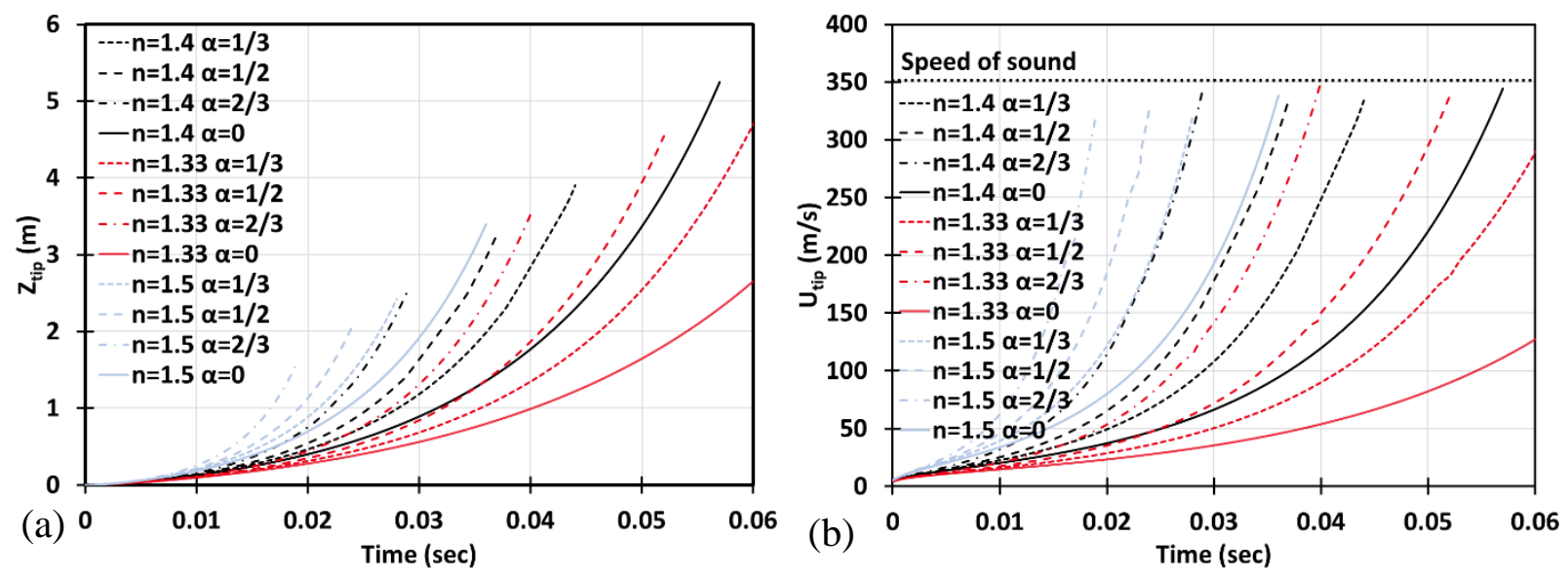

Figure 65: Time evolution of the flame tip position $Z_{t i p}$ (a) and velocity $U_{t i p}$ (b) in a cylindricalaxisymmetric geometry for stoichiometric $\mathrm{CH}_{4}$-air burning with various blockage ratios $\alpha=$ $0,1 / 3,1 / 2,2 / 3$ and various power factors $n=1.33,1.4,1.5$.

Next, the stoichiometric $(\phi=1)$ gaseous methane-air combustion considered in Fig. 64 was extended to the equivalence ratios in the range $0.6 \leq \phi \leq 1.4$; see Figs. 66-67. Figures 66 (a, b) are, respectively, the counterparts of Figs. $64(\mathrm{a}, \mathrm{b})$ for the methane-air mixtures of various equivalence ratios: $\phi=0.8,1.0,1.2$. It is observed here that a fuel-lean flame with $\phi=0.8$ accelerated much slower than the $\phi \geq 1$ flames, especially in the cases of $\alpha=1 / 3$ and $1 / 2$. This was because of a much lower $U_{f}$ (and thereby higher $L_{f}$ and lower $k_{D L}$ ) inherent to such a fuellean condition. However, it is recalled that flame acceleration in an obstructed passage is unlimited in time and, therefore, it may eventually trigger the DDT provided sufficiently long passage and time. In particular, an approximate time of a detonation initiation for the $\phi=0.8$ flame was predicted as $t_{\text {rud }} \sim 0.1172 \mathrm{~s}$ for $\alpha=1 / 3$ and $t_{\text {rud }} \sim 0.0986 \mathrm{~s}$ for $\alpha=1 / 2$. Overall, among all equivalence ratios considered, fastest flame acceleration was observed for a slightly fuel-rich flame of $\phi \sim 1.1$. 

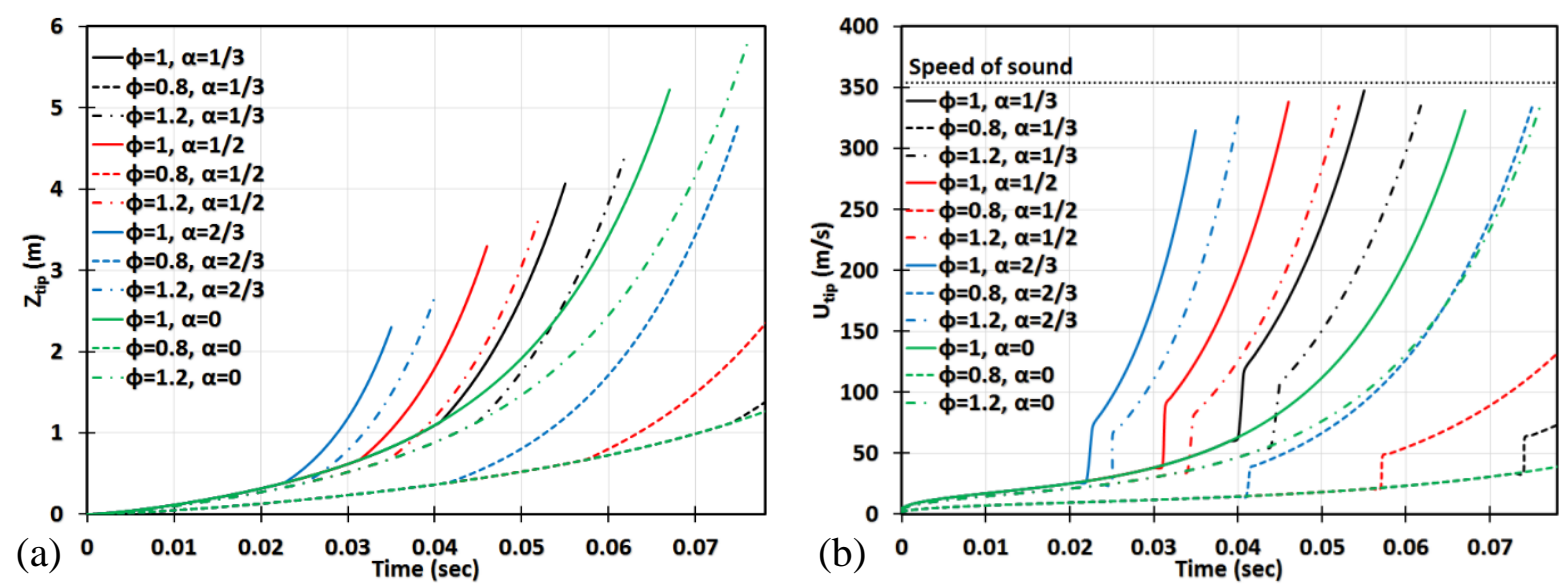

Figure 66: Evolution of the flame tip position $Z_{\text {tip }}$ (a) and velocity $U_{\text {tip }}$ (b) for the lean $(\phi=0.8$ ), stoichiometric $(\phi=1)$, and rich $(\phi=1.2)$ methane-air mixtures with various $\alpha=0,1 / 3,1 / 2,2 / 3$.

Again, further away from stoichiometry, Fig. 67, the $\phi=0.6$ and $\phi=1.4$ flames accelerate slower and the acceleration time lasts longer as compared to the $\phi=0.8 \sim 1.2$ flames in Fig. 66 . For example, in the case of $\alpha=0$, the $\phi=0.6$ flame stopped accelerating when advancing $11.2 \mathrm{~m}$ and reaching the maximal velocity of $84.7 \mathrm{~m} / \mathrm{s}$ by the instant when the flame skirt contacted the side wall of the cylindrical passage. Similar to the 2D geometry, the highly rich flame $(\phi=1.4)$ accelerated faster than the highly lean ones $(\phi=0.6)$ in the cylindrical case, and the impact of obstacles seemed to be more important for the $\phi=0.6$ flames (the lines corresponding to various blockage ratios went more widely).
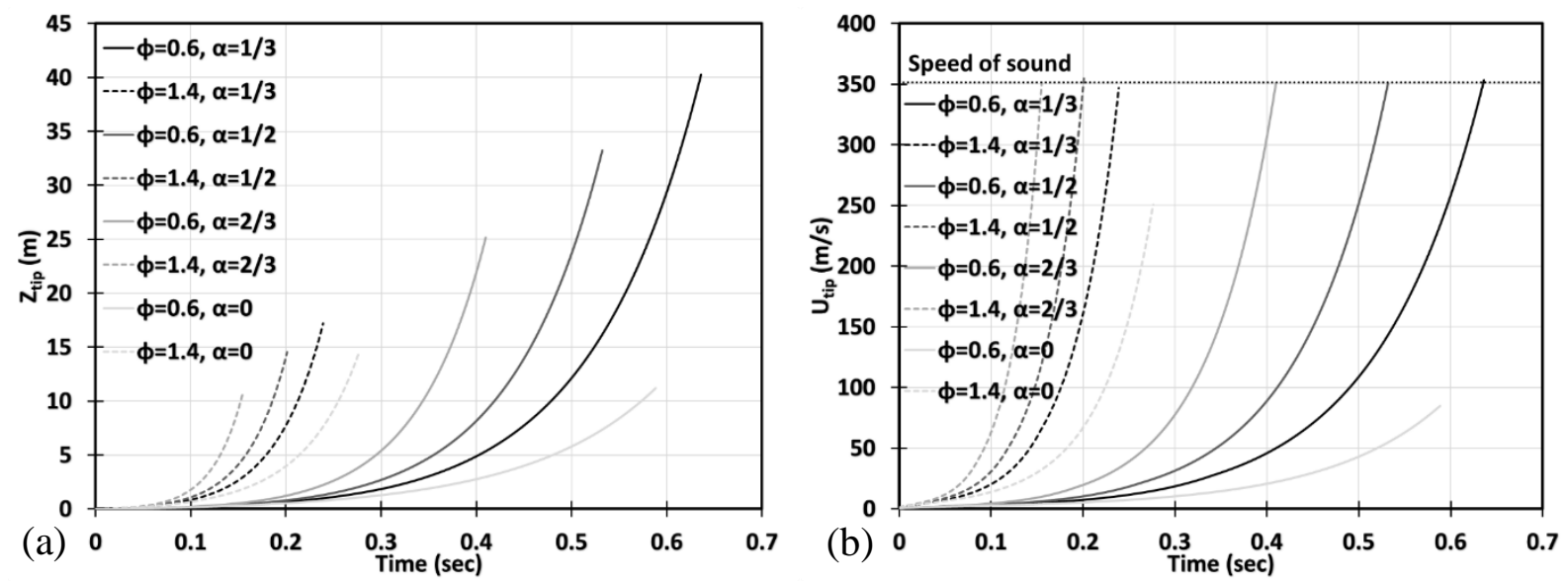

Figure 67: Time evolution of the flame tip position $Z_{t i p}$ (a) and velocity $U_{t i p}$ (b) in a cylindricalaxisymmetric geometry for highly-lean $(\phi=0.6)$ and highly-rich $(\phi=1.4) \mathrm{CH}_{4}$-air burning with various blockage ratios $\alpha=0,1 / 3,1 / 2,2 / 3$. 


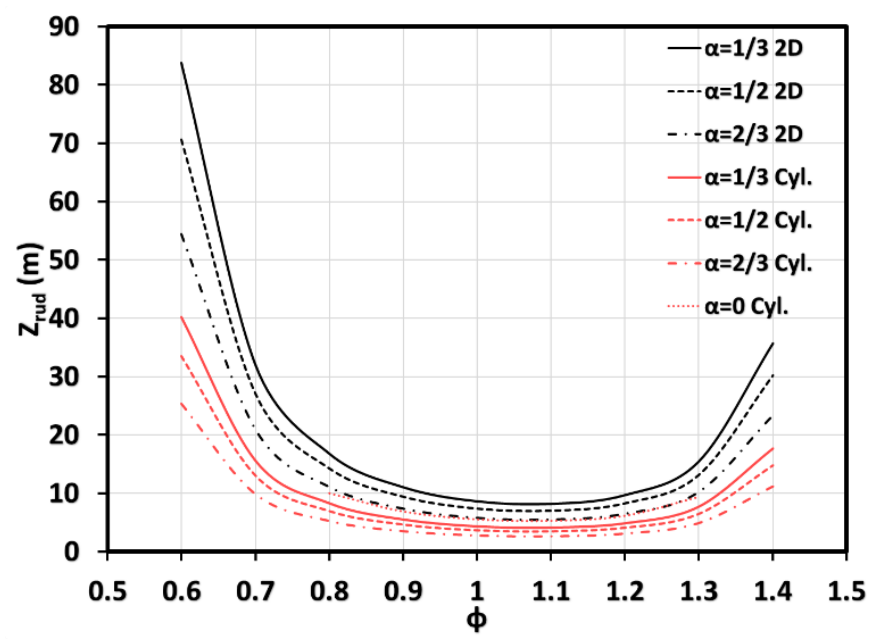

Figure 68: The flame run-up distance versus the equivalence ratio $\phi$ of a methane-air mixture at various blockage ratios: $\alpha=0,1 / 3,1 / 2,2 / 3$.

Figure 68 depicts the flame run-up distance for various equivalence and blockage ratios in both 2D and cylindrical geometries. Specifically, $X_{\text {rud }}$ and $Z_{r u d}$ calculated by Eqs. (4.14), (4.16), (4.40) and (4.41) are plotted versus $\phi$ for various $\alpha=0,1 / 3,1 / 2$ and $2 / 3$.

First, in a $2 \mathrm{D}$ geometry, the case of no obstacles, $\alpha=0$, was not relevant here because the flame skirt contacted the sidewall and stopped acceleration before the DDT event for all $\phi$ considered, which agrees with the finding of [182]. Fig. 68 agrees with the analysis in that the fastest DDT (the shortest run-up distance) occurred for a slightly fuel-rich methane-air mixture of $\phi \sim 1.1$, with $X_{\text {rud }} \sim 7.34 \mathrm{~m}, 6.68 \mathrm{~m}, 5.37 \mathrm{~m}$ for $\alpha=1 / 3,1 / 2,2 / 3$, respectively. For a lean or rich mixture, the run-up distances were much higher: up to $\sim 80 \mathrm{~m}$ for $\phi=0.6$ and up to $\sim 35 \mathrm{~m}$ for $\phi=1.4$.

Secondly, Fig. 68 also presents $Z_{\text {rud }}$ versus $\phi$ for various blockage ratios considered in cylindrical geometry, including the case of no obstacles, $\alpha=0$. Overall, Fig. 68 agrees with the analysis above in that the shortest run-up distances were observed for a slightly fuel-rich methane-air mixture of $\phi \sim 1.1$ in cylindrical geometry as well, with $Z_{\text {rud }} \sim 5.47 \mathrm{~m}, 4.11 \mathrm{~m}, 3.45 \mathrm{~m}, 2.64 \mathrm{~m}$ for $\alpha=0,1 / 3,1 / 2,2 / 3$, respectively. For the lean or rich mixtures, the run-up distances were much higher: up to $\sim 40 \mathrm{~m}$ for $\phi=0.6$ and up to $\sim 18 \mathrm{~m}$ for $\phi=1.4$. In the case of $\alpha=0$, the DDT occurred for the equivalence ratios in the range $0.8 \leq \phi \leq 1.3$; while in a leaner or richer mixture a flame skirt contacted the sidewall and a flame started decelerating agreeing with the findings $[182,200]$. 
Finally, the analysis was extended from a purely gaseous environment to a gaseous-dusty environment by using a modified Seshadri formulation for the laminar burning velocity $U_{d, f}$ as a function of the thermal-chemical properties of the gas and dust particles, Eq. (4.17). The combustible (e.g. coal) and inert (e.g. sand) particles as well as their combinations are considered. As of now, all the particles were assumed to be distributed uniformly inside the passage, while non-uniform dust distributions in a coalmine can be considered in future. Figure 69 depicts the situation of gaseous-dusty combustion, with the dust of concentration $c_{S}=50 \mathrm{~g} / \mathrm{m}^{3}$ and of dust particles radius $r_{s}=75 \mu \mathrm{m}$ in the cylindrical-axisymmetric (c, d) geometry. Lean $(\phi=0.7)$ methane-air fuel mixture and various blockage ratios $\alpha$ were employed, including the case of no obstacles, $\alpha=0$. It is seen that combined (combustible+inert) and inert dust moderated flame acceleration whereas combustible particles slightly facilitated flame propagation. The effect of obstacles was noticeable as well (compare with an unobstructed passage).
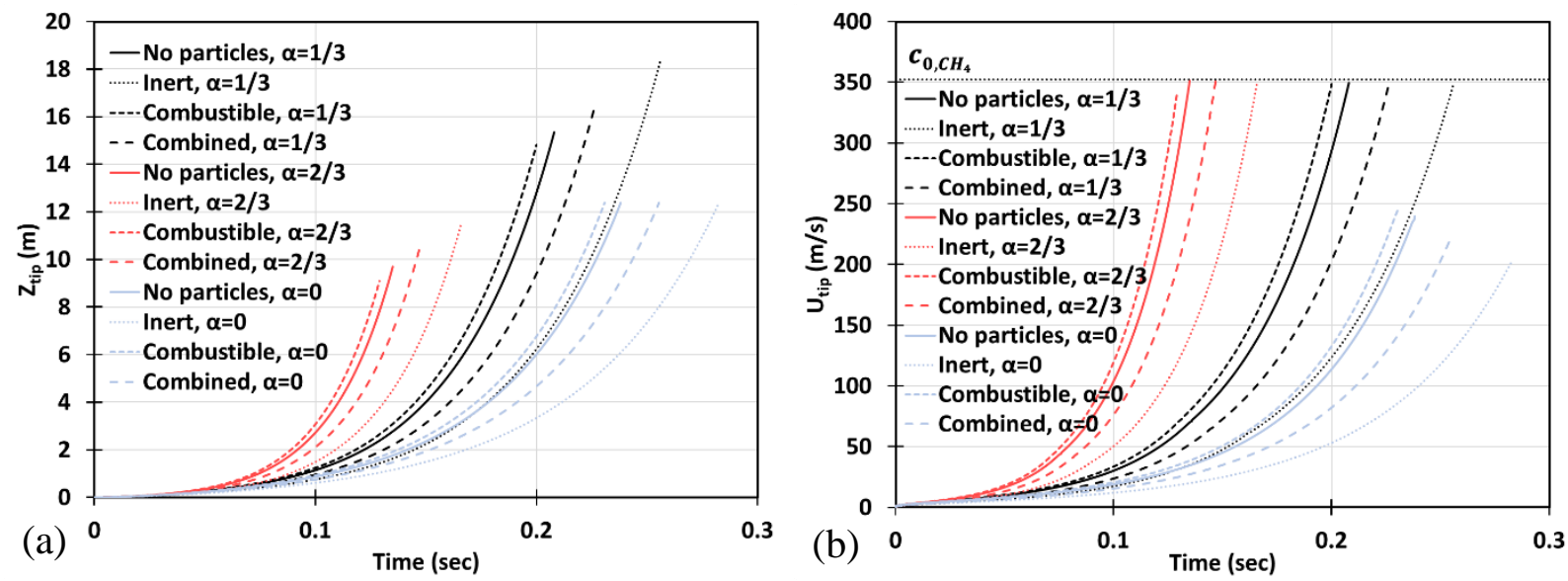

Figure 69: Time evolution of the flame tip position $Z_{t i p}$ (a) and velocity $U_{t i p}$ (b) in a cylindricalaxisymmetric geometry for lean $\mathrm{CH}_{4}$-air burning of $\phi=0.7$, without and with dust particles (combustible, inert, and combined) of particle radius $\boldsymbol{r}_{\boldsymbol{s}}=\mathbf{7 5} \boldsymbol{\mu \mathrm { m }}$ and concentration $\boldsymbol{c}_{\boldsymbol{s}}=\mathbf{5 0} \mathrm{g} / \mathbf{m}^{\mathbf{3}}$, for various blockage ratios: $\alpha=0,1 / 3,2 / 3$.

Next, the dust concentration was increased. Namely, in Fig. 70, it is promoted to $c_{s}=120 \mathrm{~g} / \mathrm{m}^{3}$ keeping the same particle radius, $r_{s}=75 \mu \mathrm{m}$, and other characteristic and geometry as in Fig. 69. It is observed that the combustible dust facilitated flame acceleration, while the inert dust and its combination with the combustible dust moderated the acceleration process for the radius of the dust particles being $r_{s}=75 \mu \mathrm{m}$. The effect of blockage ratio was noticeable in that the rate of burning with inert particles for $\alpha=2 / 3$ was equivalent to that of combustible dust-gaseous burning for the case of no obstacles, $\alpha=0$, for $t>0.136 \mathrm{~s}$, and then faster burning was found. 


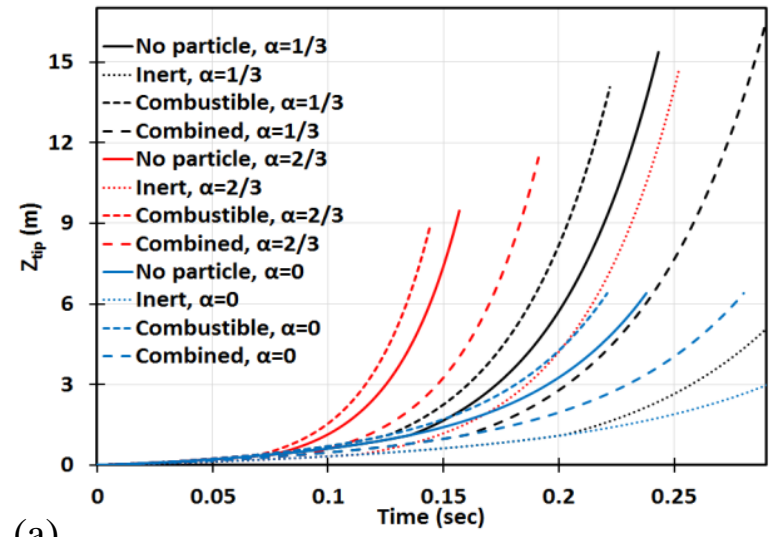

(a)

Figure 70: Evolution of the flame tip position $Z_{\text {tip }}$

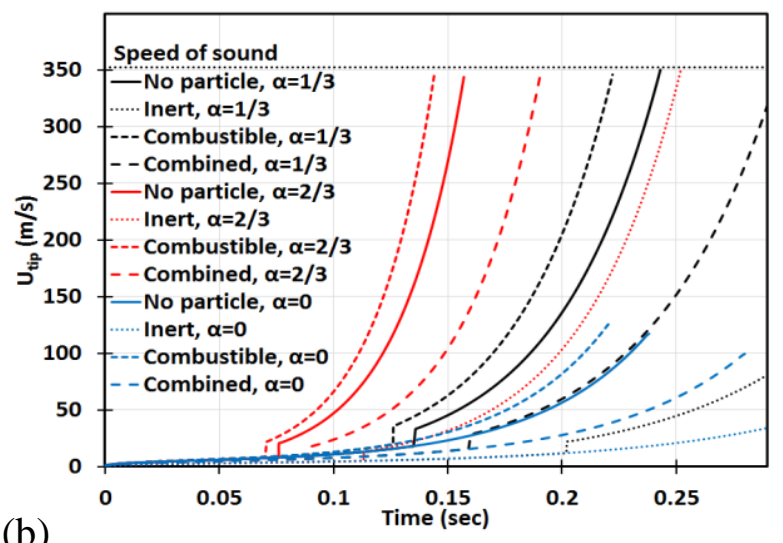

(b) concentration $\boldsymbol{c}_{\boldsymbol{s}}=\mathbf{1 2 0} \mathbf{g} / \mathbf{m}^{3}$, for various blockage ratios: $\alpha=0,1 / 3,2 / 3$.

Relatively high concentration has also been considered. Specifically, Fig. 71 presents the case of $c_{s}=250 \mathrm{~g} / \mathrm{m}^{3}$, with the particle size $r_{s}=75 \mu \mathrm{m}$, the same as in Figs. 69, 70. This investigation revealed that if keeping the dust particle size constant while increasing the number of particles (i.e. the concentration), the effect of particles became more important. This was observed from the Figs. 69-71 that the flames in gaseous-dusty environments departed from the case of no dust particles more widely as the concentration increased. Similar to the cases of $c_{S}=50$ and $120 \mathrm{~g} / \mathrm{m}^{3}$, the combustible particles promoted flame acceleration whereas the combined (combustible+inert) and inert particles suppressed it in the situation of $c_{s}=250 \mathrm{~g} / \mathrm{m}^{3}$ as well.
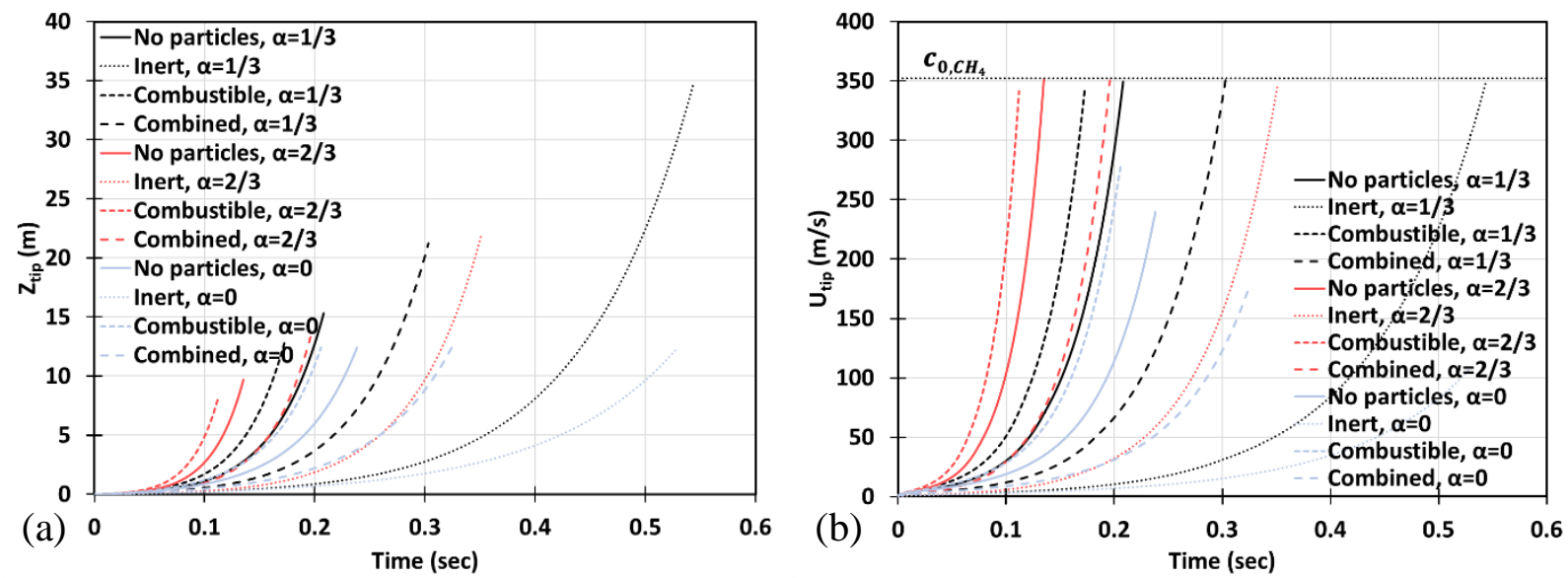

Figure 71: Time evolution of the flame tip position $Z_{\text {tip }}$ (a) and velocity $U_{\text {tip }}$ (b) in a cylindricalaxisymmetric geometry for lean $\mathrm{CH}_{4}$-air burning of $\phi=0.7$, without and with dust particles

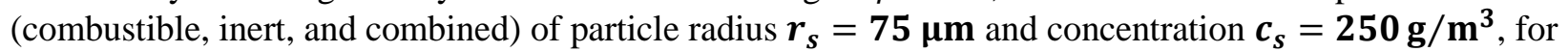
various blockage ratios: $\alpha=0,1 / 3,2 / 3$. 
Figures 72 (a, b) are, respectively, the counterparts of Figs. 70 (a, b) for a smaller radius of the dust particles, $r_{s}=10 \mu \mathrm{m}$. It is observed here that smaller particles had a stronger impact on flame propagation. Specifically, the coal particles deviated noticeably larger from the case of no particles. In fact, the particle type appeared as important factor as the blockage ratio in terms of influencing flame acceleration in obstructed passages. Namely, in any case of the combustible dust mixtures in the obstructed passages considered, i.e. for $\alpha=0,1 / 3,2 / 3$, faster acceleration was obtained than in all respective cases of no dust particles. These outcomes were interesting from the viewpoint of the same particle concentration used in Figs. 70 and 72: it can be concluded that the larger number of particles but of smaller size provided stronger flame acceleration. This statement was also supported by the trend of combined (combustible + inert) particles of radius $r_{s}=10 \mu \mathrm{m}$. Opposite to the case of $r_{s}=75 \mu \mathrm{m}$ in Fig. 70, the combined particles of radius $r_{s}=$ $10 \mu \mathrm{m}$ promoted flame acceleration. These findings show that the impact of a heat release on flame acceleration in a coalmining passage was significant: it facilitated the fire process, and, furthermore, it dominated over the impact of a heat sink when the particle size was smaller. As for the inert particles, similarly to the case of $r_{s}=75 \mu \mathrm{m}$ in Fig. 70, they suppressed flame acceleration for $r_{s}=10 \mu \mathrm{m}$ as well. The aforementioned effects of all particles, i.e. combustible, inert, and combined, and particle sizes were seen to get larger with $\alpha$.
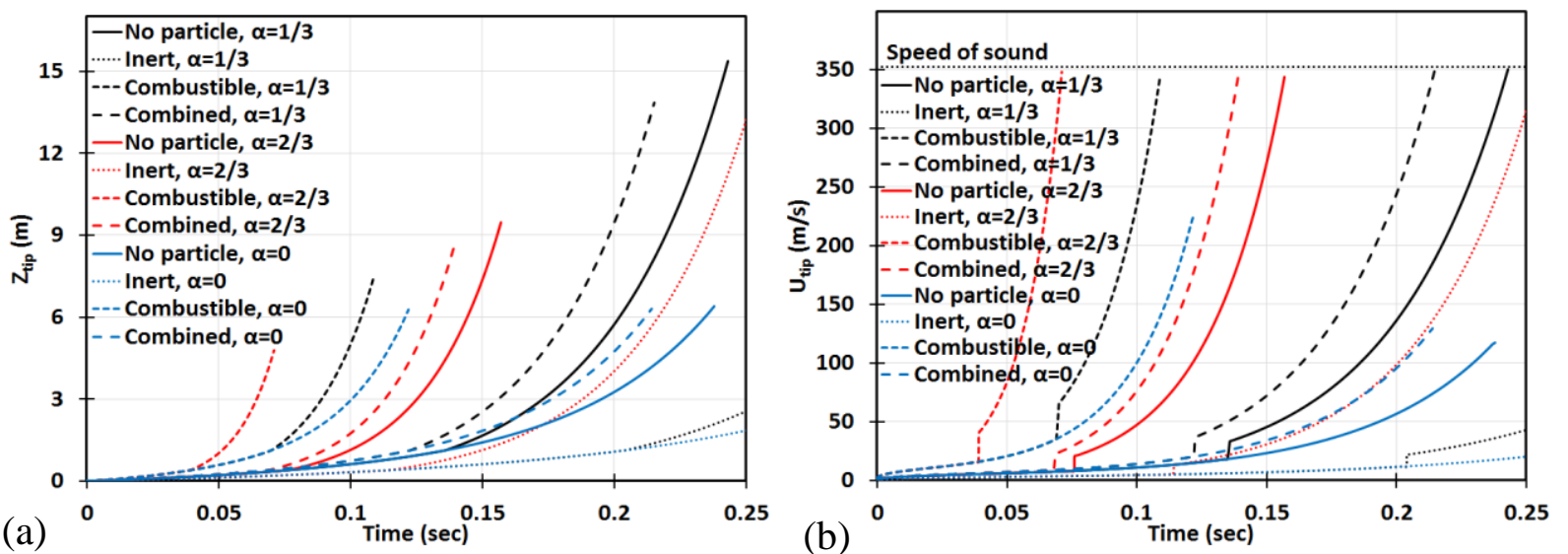

Figure 72: Evolution of the flame tip position $Z_{t i p}$ (a) and velocity $U_{t i p}$ (b) for a lean methane-air mixture of $\phi=0.7$ with and without dust particles (inert, combustible, and combined) of radius $\boldsymbol{r}_{\boldsymbol{s}}=\mathbf{1 0} \boldsymbol{\mu m}$ and concentration $\boldsymbol{c}_{\boldsymbol{s}}=\mathbf{1 2 0} \mathbf{g} / \mathbf{m}^{\mathbf{3}}$, for various blockage ratios: $\alpha=0,1 / 3,2 / 3$. 


\subsubsection{Incorporation of Compressibility}

The formulation derived above predicts unlimited acceleration, which may promote the burning velocity to near-sonic values, for which the incompressible model is not acceptable. To explain such a discrepancy, the formulation was next extended to account for small but finite Mach numbers associated with flame propagation, $M a \ll 1$. Such an assumption of a small $M a$ indicates that the temperature and density of a generally multicomponent ideal gas can be computed in terms of the gas composition at a given constant reference pressure [228]. As a result, the chemistry appears independent of the local pressure, which persists a kinematic variable [229]. The methodology is similar to that of Refs. [230, 231]. However, while such a Mach number was constant in Refs. [230, 231] ( $M a \equiv U_{f} / c_{0}$, with $c_{0}$ being the initial speed of sound in the fresh mixture), here it is a transient quantity, $\operatorname{Ma}(t)=U_{D L}(t) / c_{0}$. As long as the burning process is substantially subsonic, $M a \ll 1$, the flow in the unburnt gas (subscript "u") can be treated as isentropic, with the instantaneous density, pressure, and temperature given by (see Refs. [230,231] for more details)

$$
\begin{aligned}
& \frac{\rho_{u}(t)}{\rho_{0}}=\left(1+\frac{\gamma-1}{2} \frac{u_{z, 1}(t)}{c_{0}}\right)^{2 /(\gamma-1)} \approx 1+M a(t)(\Theta-1)\left(1+\frac{Z_{t i p}(t)}{h}\right), \\
& \frac{P_{u}(t)}{P_{0}}=\left(1+\frac{\gamma-1}{2} \frac{u_{z, 1}(t)}{c_{0}}\right)^{2 \gamma /(\gamma-1)} \approx 1+\gamma M a(t)(\Theta-1)\left(1+\frac{Z_{t i p}(t)}{h}\right), \\
& \frac{T_{u}(t)}{T_{0}}=\left(1+\frac{\gamma-1}{2} \frac{u_{z, 1}(t)}{c_{0}}\right)^{2} \approx 1+(\gamma-1) M a(t)(\Theta-1)\left(1+\frac{Z_{t i p}(t)}{h}\right),
\end{aligned}
$$

where $\gamma=c_{p} / c_{v} \approx 1.4$ is the adiabatic index and $\rho_{0}, P_{0}, T_{0}$ are the initial values in the unburnt gas. The quantity $u_{z, 1}$ denotes the axial flow velocity at the flame front, at its "unburnt side." Later, its counterpart at the "burnt side" of the front, $u_{z, 2}$, will also be considered. It is noted that while the index " 0 " designates the initial, incompressible values, the indexes " 1 " and "2" are devoted to the transient, instantaneous quantities. In particular, instead of the initial thermal expansion ratio, $\Theta$, the analysis deals with an instantaneous (reduced) expansion ratio

$$
\vartheta(t)=\Theta-(\gamma-1) M a(t)(\Theta-1)\left\{1+Z_{t i p}(t) / h\right\} .
$$

Equation (4.45) has been derived accounting for a small but finite $M a$ such that the zeroth- and first-order terms in $M a$ have been counted, while the second-order and higher order terms in $M a$ 
have been neglected (see Refs. [230, 231] for more details of this part of the derivation). Consequently, the value of $M a$ will be the measure of the validity of the present formulation. Overall, Eq. (4.45) shows that the thermal expansion ratio, accounting for compressibility, $\vartheta$ is less than the incompressible quantity $\Theta$, with $\left.\vartheta\right|_{M a=0}=\Theta$. The 2D continuity equation for small but finite compressibility

$$
\nabla \mathbf{u}=-\left(\partial P_{u} / d t\right) / \gamma P_{u}
$$

has the following solution in the burnt gas:

$$
\begin{aligned}
& u_{x}=-\frac{1}{\gamma P_{u}} \frac{\partial P_{u}}{\partial t} \frac{x}{h} \\
& u_{z}=\left(\frac{1}{\gamma P_{u}} \frac{\partial P_{u}}{\partial t}+(\vartheta-1) \frac{U_{D L}(t)}{h}\right) z .
\end{aligned}
$$

Substituting Eqs. (4.45) - (4.48) into modified Eq. (4.10),

$$
Z_{t i p}-u_{z, 2}(\vartheta(t))=\vartheta(t) U_{D L}(t)
$$

[it is recalled that $u_{z, 2}$ in Eq. (4.49) is the axial flow velocity at the "burnt side" of the flame front, while $u_{z, 1}$ is that at the "unburnt side"], and further neglecting the $2^{\text {nd }}$ and higher order terms in $M a$, the final evolution equation for the flame tip is eventually obtained,

$$
\frac{d Z_{t i p}}{d t}=-\chi \frac{\sigma_{0}^{2}}{U_{D L}(t)} Z_{f}^{2}+\sigma_{1} Z_{f}+\Theta_{1} U_{D L}(t),
$$

where $\sigma_{0}=(\Theta-1) U_{D L}(t) /(1-\alpha) H$ is the exponential factor in Eq. (4.11) and

$$
\begin{gathered}
\chi=M a(t)\left[(1-\alpha)^{-1}+\gamma-1\right]+\frac{d M a(t)}{d t} \frac{R}{(\Theta-1) U_{D L}(t)}, \\
\sigma_{1}=\sigma_{0}\left[1-M a(t)\left(\frac{\Theta}{(1-\alpha)}+2(\gamma-1)(\Theta-1)\right)-\frac{d M a(t)}{d t} \frac{R}{U_{D L}(t)}\right], \\
\Theta_{1}=\Theta-M a(t)(\gamma-1)(\Theta-1)^{2} .
\end{gathered}
$$

Similar to the incompressible approach, the compressible formulation, Eq. (4.50), is solved until the instant, $t_{o b s}$, after which the Mach number associated with flame propagation, $M a\left(t_{o b s}\right)$ and the instantaneous global flame front velocity $U_{D L}\left(t_{o b s}\right)$ become constants. With the corresponding flame tip position $Z_{t i p, c}\left(t_{o b s}\right)$ for $t>t_{o b s}$, Eq. (4.50) is integrated as

$$
Z_{\text {tip }}=\frac{Z_{t i p, 2} C_{1} \exp \left[\sigma_{2}\left(t-t_{o b s}\right)\right]-Z_{t i p, 1}}{C_{1} \exp \left[\sigma_{2}\left(t-t_{o b s}\right)\right]-1}
$$


where

$$
\begin{gathered}
\sigma_{2}=\sqrt{\sigma_{1}^{2}+4 M a \Theta_{1} \chi \sigma_{0}^{2}}, \\
C_{1}=\left(Z_{t i p, c}\left(t_{o b s}\right)-Z_{t i p, 1}\right) /\left(Z_{t i p, c}\left(t_{o b s}\right)-Z_{t i p, 2}\right),
\end{gathered}
$$

with

$$
Z_{t i p, 1}=\left(\sigma_{1}-\sigma_{2}\right) /\left(2 M a \chi \sigma_{0}^{2} / U_{D L}\right) \text { and } Z_{t i p, 2}=\left(\sigma_{1}+\sigma_{2}\right) /\left(2 M a \chi \sigma_{0}^{2} / U_{D L}\right)
$$

keeping in mind that $M a=M a\left(t_{o b s}\right)$ and $U_{D L}=U_{D L}\left(t_{o b s}\right)$.

\subsubsection{Results and Discussion in a Compressible 2D Geometry}

Fig. 73 presents the results obtained for stoichiometric, $\phi=1$, methane-air combustion. Namely, Fig. 73a shows the evolution of flame tip position while Fig. $73 \mathrm{~b}$ is for the flame tip position. It was seen that the compressible theory agrees very well with the incompressible formulation at the early stages of methane-air burning accident. However, the effect of compression comes to play later on. mitigating the flame propagation velocity as compared to the incompressible approach. Physically, the mechanism for moderation of flame acceleration due to gas compressibility is the following. An accelerating flame acts as a "piston," generating and pushing the compression waves. These waves compress the fuel mixture, thereby elevating its initial temperature. The temperature of the burnt matter will also be elevated, but the burnt to unburnt gas temperature ratio (defining the thermal expansion ratio, $\vartheta$ ) will therefore be reduced as compared to its initial value $\left.\vartheta\right|_{M a=0}=\Theta$, see Eq. (4.45). It is recalled, in this respect, that flame acceleration occurs due to thermal expansion of the burning gas in the combustion process (indeed, a newly generated volume of the gas has to be moved out; say, in the artificial limit of no thermal expansion, $\vartheta=\Theta=1$, there would be no flame acceleration). Consequently, the reduction in the thermal expansion ratio moderates flame acceleration.

From the mathematical interpretation point of view, the first nonlinear term becomes quite important soon and suppresses the flame acceleration trend, from an exponential one, according to the incompressible theory, to slower linear acceleration. Moreover, the effect of the nonlinear term in Eq. (4.50) breaks the compressible theory for all blockage ratios later, or especially for relatively large blockage ratios, i.e. $\alpha \geq 1 / 2$, earlier such that the flame deviates from incompressible theory quite soon and starts to slowdown. For this reason, a better validity range of both theory for when $\alpha=0$, i.e. the channel is unobstructed, or $\alpha=1 / 3$ was selected for the rest of this study. From a 
practical viewpoint, the blockage ratios of $\alpha=1 / 2$ and 2/3 are unlikely, however, still needs to be taken into consideration in the worst-case predictions.
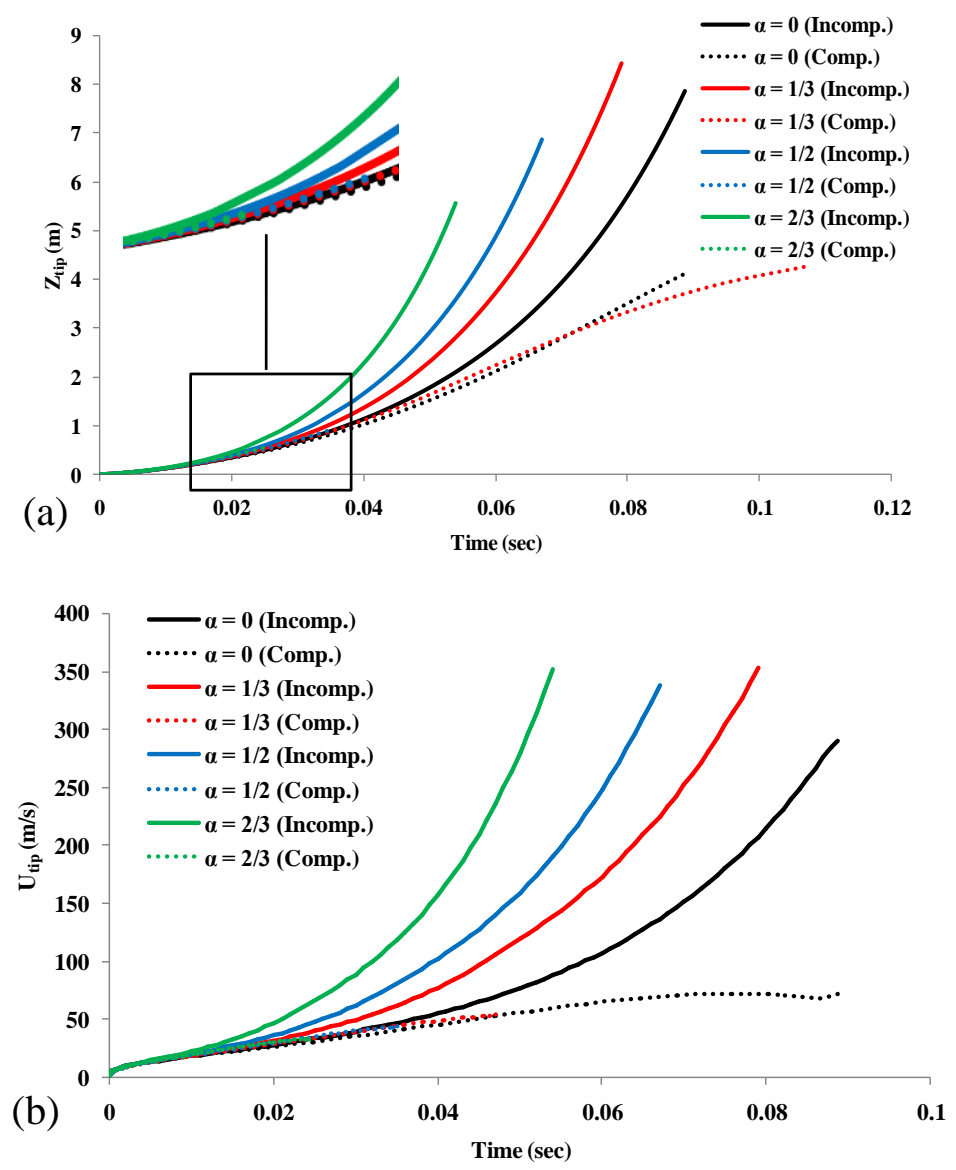

Figure 73: Evolution of flame tip position, $Z_{\text {tip }}$ (a), and velocity, $U_{\text {tip }}(\mathrm{b})$, for stoichiometric $\mathrm{CH}_{4}$-air burning with various mining blockage ratios considered: $\alpha=0,1 / 3,1 / 2,2 / 3$.

Figures $74 \mathrm{a}$ and $74 \mathrm{~b}$ are the counterparts of Figs. $73 \mathrm{a}$ and $73 \mathrm{~b}$ but with a fixed blockage ratio, $\alpha=$ $1 / 3$, and a variety of equivalence ratios considered: $\phi=0.8$ (lean), $\phi=1$ (stoichiometric) and $\phi=1.2$ (rich). It is seen that the gas compression moderates flame acceleration, and this effect is stronger for rich or stoichiometric methane-air conditions. In the fuel-lean mixture, compressible and incompressible theory agrees well at the initial stage of the combustion, however, both trends deviate noticeably at a later stage of the process. Indeed, here the effect of gas compression dominates over that of flame acceleration and reduces the flame velocity, driving to a conclusion that Eq. (4.54) might over-predict the impact of gas compression. 

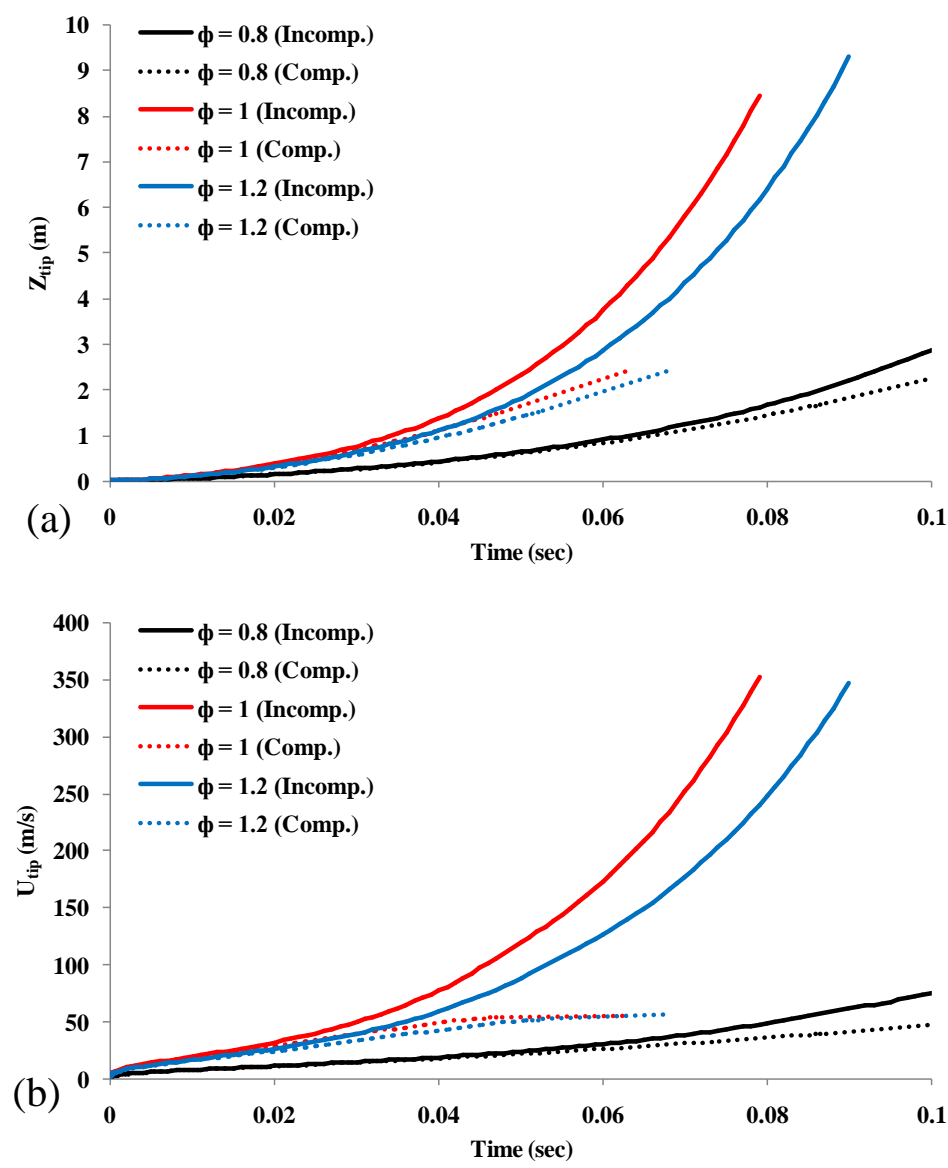

Figure 74: Evolution of flame tip position, $Z_{t i p}$, (a) and velocity, $U_{t i p}$, (b) of a $\mathrm{CH}_{4}$-air flame at various equivalence ratios: $\phi=0.8 ; 1$ and 1.2, with a fixed blockage ratio of $\alpha=1 / 3$.

Next the impact of a blockage ratio, $\alpha$, within the frame of the compressible formulation was scrutinized. Namely, the blockage ratio $\alpha=1 / 3$ is compared to the case of unobstructed channel condition $\alpha=0$. Specifically, corresponding flame tip positions are plotted in Fig. 75 for $\phi=$ $0.8 ; 1 ; 1.2$ methane-air combustion. Unlike the incompressible theory, the effect of the blockage ratio appears minor within the frame of the compressible theory. This can be seen when comparing Fig. 75 to stoichiometric methane-air flame (Fig. 73a) with $\alpha=0$ and 1/3. Moreover, Fig. 74 also shows that as the mixture gets closer to stoichiometry, the effect of the blockage is greatly diminished as compared with that of the fuel-lean mixture. This is due to the fact that the nonlinear term in Eq. (4.50) and the second term in Eq. (4.53) become dominant rapidly as methane-air flame parameters such as thermal expansion ratio $\Theta$ and the instantaneous global flame speed $U_{D L}$ are increased at mixture conditions near stoichiometry. Such a correlation diminishes the role of the blockage ratio for "compressible" flame propagation. 


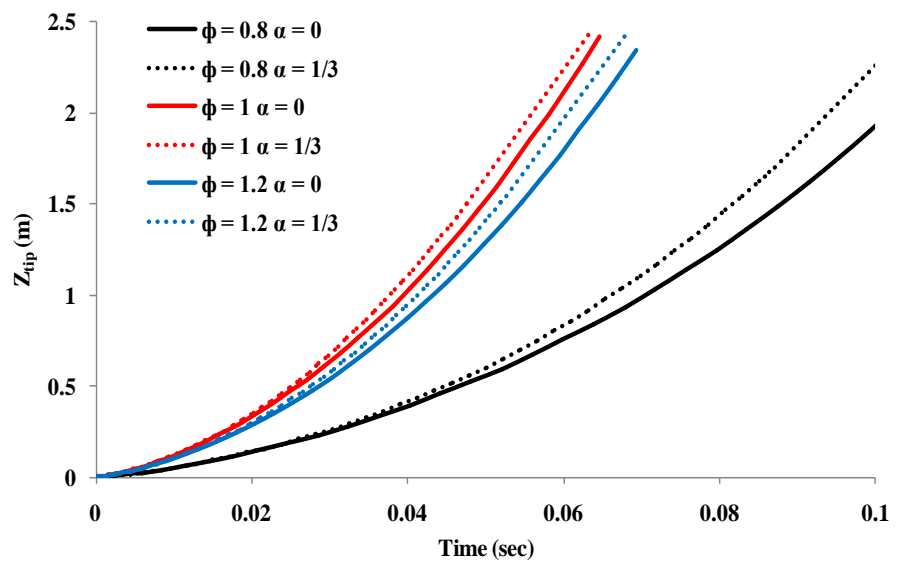

Figure 75: Evolution of the flame tip position $Z_{\text {tip }}$ in passages of blockage ratios $\alpha=0$ and $1 / 3$ for lean $(\phi=0.8)$, stoichiometric $(\phi=1)$ and rich $(\phi=1.2) \mathrm{CH}_{4}$-air burning accounting for gas compression.

Starting with homogeneous gaseous combustion, the analysis is subsequently extended to account for the presence of dust particles in the coalmining environment. On the one hand, combustible particles (such as coal dust) may release heat into a gaseous environment during their volatilization process, thereby facilitating the combustion process. On the other hand, the presence of inert dust particles (such as sand) or the heat gain process of combustible particles may moderate the process. To account for these effects, the Seshadri formulation [202] is employed to define the laminar burning velocity of gaseous-dusty environment, as explained in the Sec. 4.3.2.

Next, combustible and inert particles as well as their combinations were incorporated to consider slightly lean methane-air burning, $\phi=0.7$. Figure 76 is devoted to the cases of dust particles of radius $r_{s}=75 \mu \mathrm{m}$ and concentration $c_{d}=120 \mathrm{~g} / \mathrm{m}^{3}$, as well as to that without particles. Here, the blockage ratio is as large as $\alpha=1 / 3$. It is again seen that gas compression moderates flame acceleration in all cases. The presence of dust influences flame acceleration, specifically, while combustible dusts facilitate the combustion process, the addition of inert or combined dust reduce the flame velocity. However, it is emphasized that the deviations from the no particle case are reduced when the gas compression is incorporated in the analysis. 

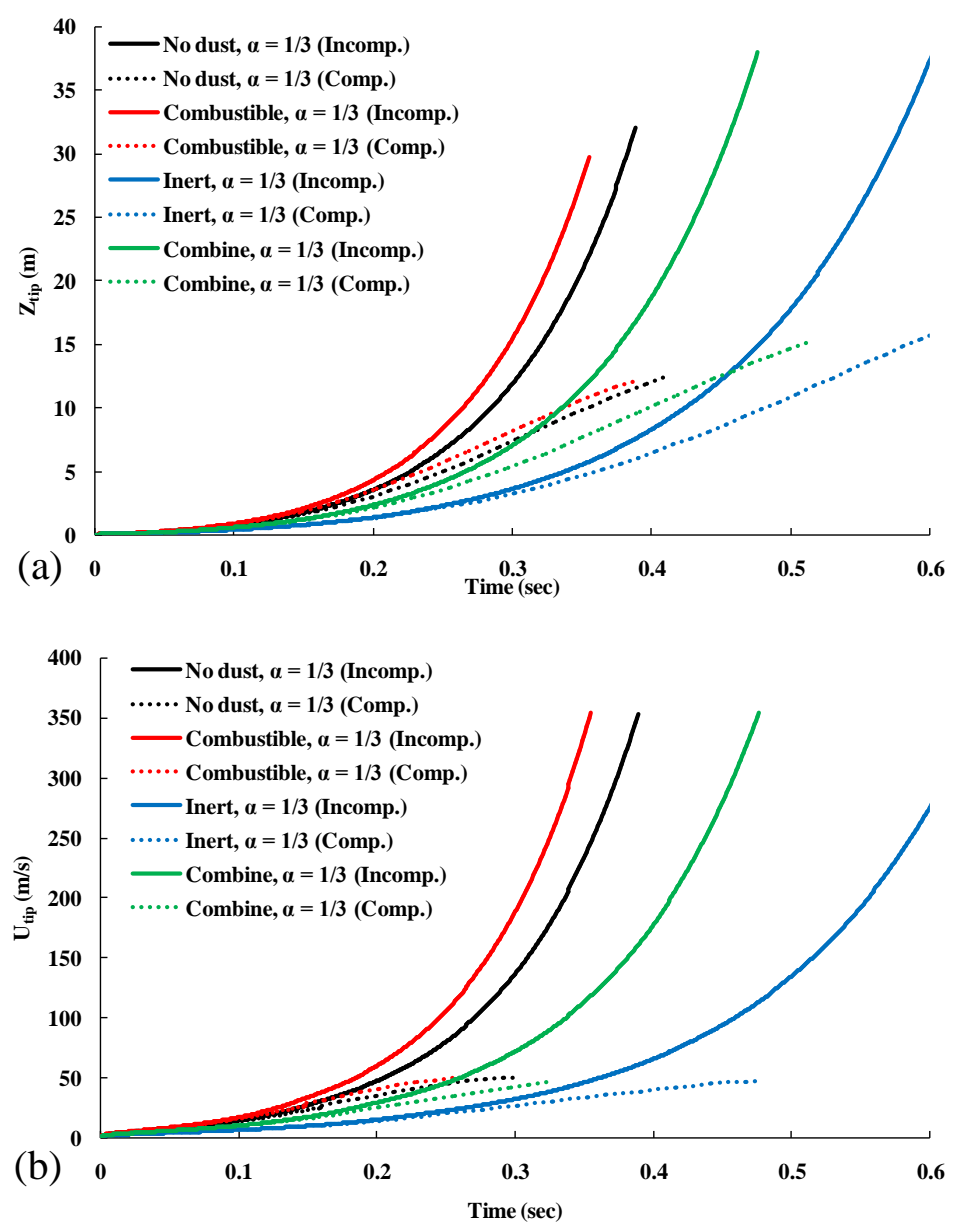

Figure 76: Evolution of flame tip position, $Z_{\text {tip }}$, (a) and velocity, $U_{\text {tip }}$, (b) for lean $(\phi=0.7) \mathrm{CH}_{4}$-air burning, with and without dust particles (inert, combustible, and combined) of radius $r_{s}=75 \mu \mathrm{m}$ and concentration $c_{d}=120 \mathrm{~g} / \mathrm{m}^{3}$ in a passage with a blockage ratio of $\alpha=1 / 3$.

Finally, the size of the particles, from $r_{s}=75 \mu \mathrm{m}$ to $r_{s}=10 \mu \mathrm{m}$ were reduced and the results are presented in Figs. 77a and 77b, for the evolution of the flame tip position, $Z_{t i p}$, and velocity, $U_{t i p}$, respectively. All other parameters such as the dust concentration as well as the blockage and equivalence ratios are kept the same as in Figs. 76a and 76b. It is seen that similar to the case of $r_{s}=75 \mu \mathrm{m}$, the influence of gas compression is also significant here. However, the plots in Fig. 77 deviate from each other noticeably. While the combustible particles facilitate flame propagation and the inert particles moderate the combustion process, it is interesting that the combined dust, in contrast to the case of $r_{s}=75 \mu \mathrm{m}$, promotes flame acceleration according to the compressible formulation. This certifies agreement between the two theories, even though not quantitatively but qualitatively. This finding shows that the particle size can influence the burning scenario and smaller particles may facilitate flame acceleration as compared with the case of larger particles. It 
is nevertheless noted that the maximal values of the flame tip velocity in Fig. 77b, obtained for the combined dust and no dust cases, are almost same in the compressible theory, but acceleration is slower without dust.
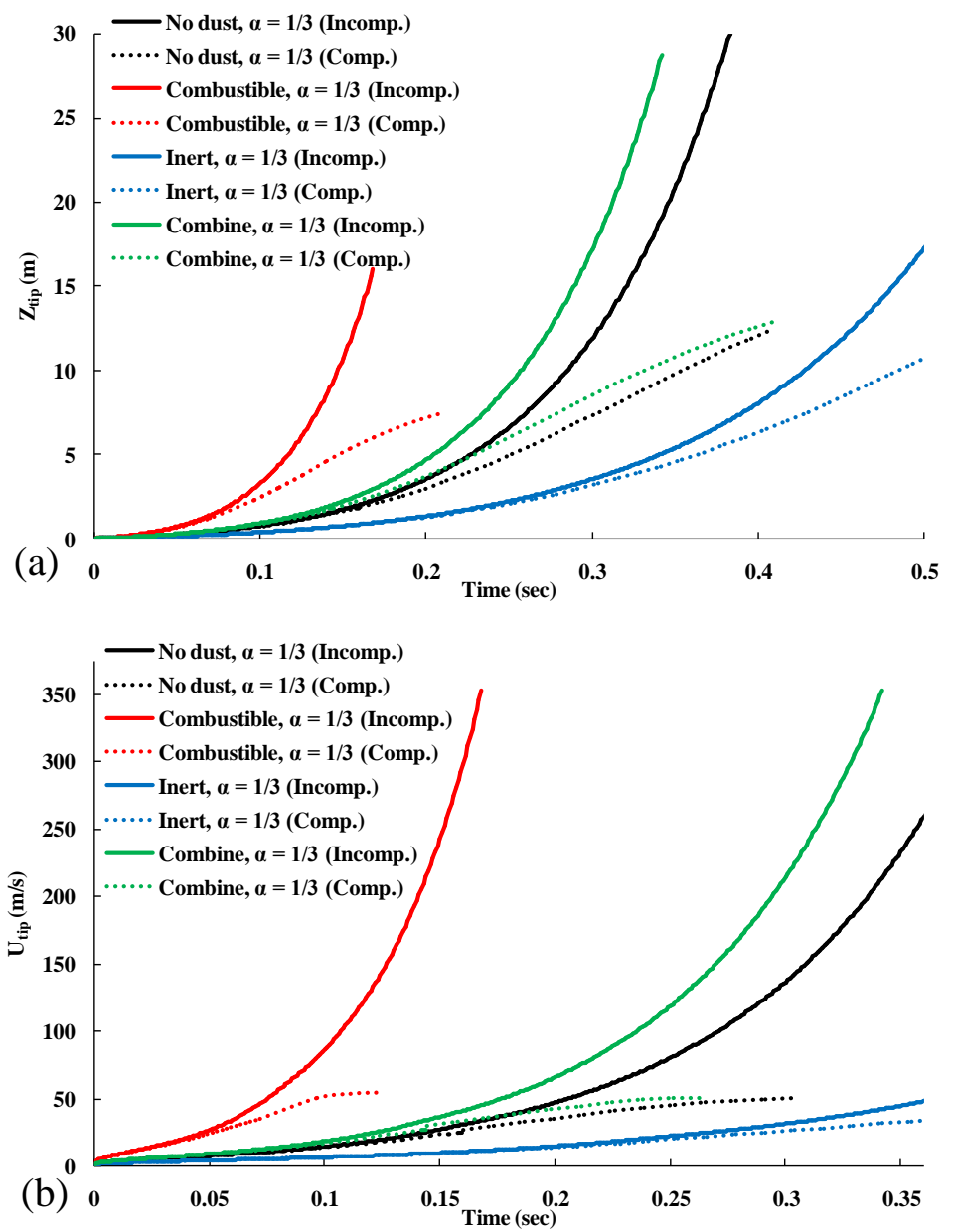

Figure 77: Evolution of flame tip position, $Z_{\text {tip }}$, (a) and velocity, $U_{\text {tip }}$, (b) for lean $(\phi=0.7)$ methane-air burning, with and without dust particles (inert, combustible, and combined) of radius $r_{s}=10 \mu \mathrm{m}$ and concentration $c_{d}=120 \mathrm{~g} / \mathrm{m}^{3}$ in a passage with a blockage ratio of $\alpha=1 / 3$.

\subsubsection{Comparison of Theory and Data in the Literature}

The analytical predictions were compared to the data available in the literature such as experiments [232] and computational simulations ALLA and FAST [208] - the two computational models that predicts the dynamics of flame acceleration and DDT. They both use the same chemical-diffusive model but implements different adaptive mesh refinements. ALLA solves the governing equations with a second-order accurate numerical method in space and the same in time. FAST solves the governing equations with a fifth-order accurate numerical method in space and third-order in time. 

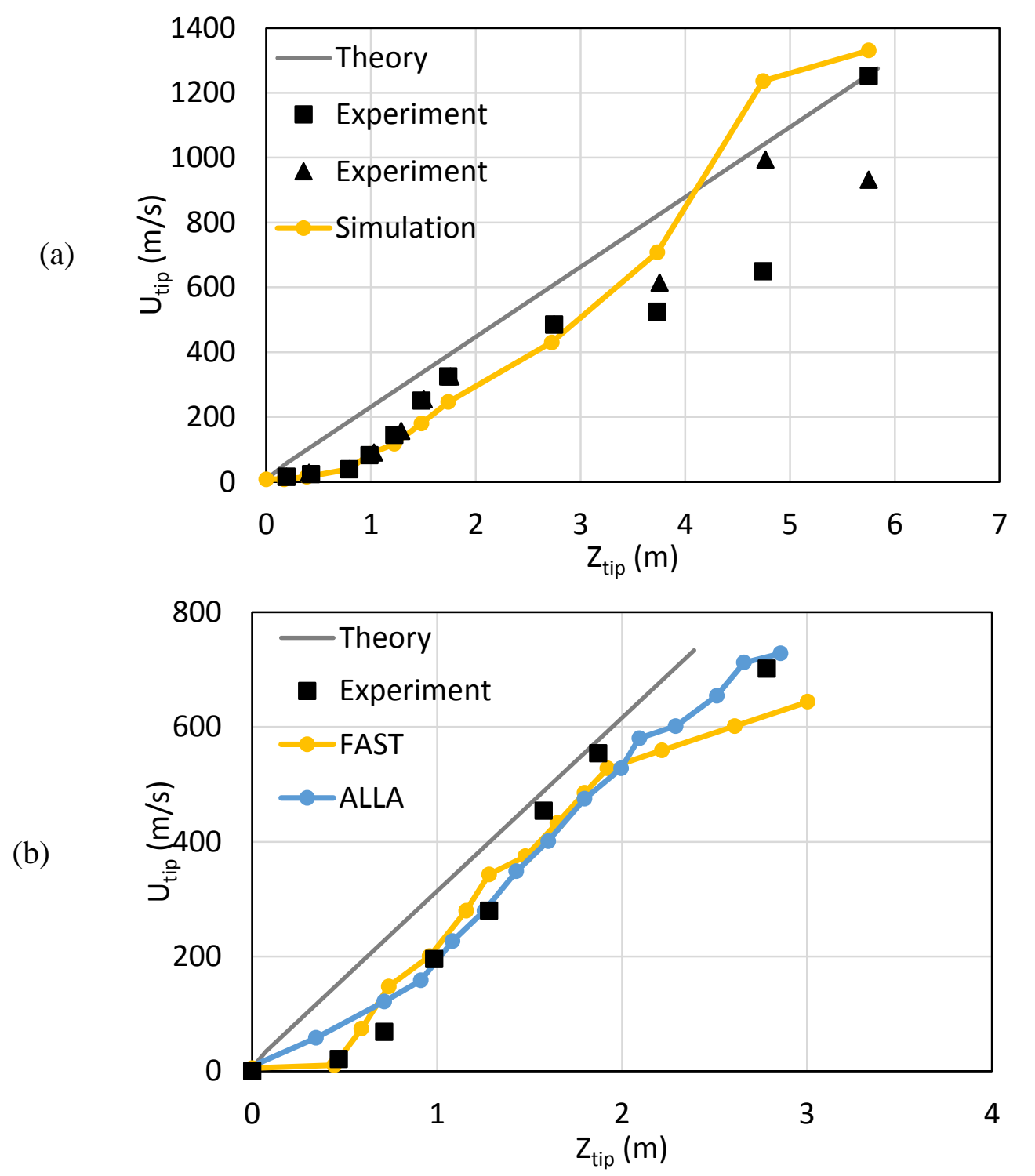

Figure 78: Comparison of theoretical formulation with the experiments [232] and numerical simulations [208] (FAST, yellow, \& ALLA, blue): the flame tip velocity vs its position for stoichiometric methane explosion in in obstructed channel with height $H=0.174 \mathrm{~m}$ and blockage ratios $\alpha=0.3$ (a) \& 0.6 (b).

The comparison is shown in Fig. 78. Here the flame speed versus flame position is plotted for stoichiometric methane explosion in the obstructed channel with height of $H=0.174 \mathrm{~m}$ and $\alpha=$ 0.3, Fig. 78a and $\alpha=0.6$, Fig. 78b. Two experiments in Fig. 78a are two repeated tests of stoichiometric methane explosions [232]. Simulation in Fig. 78a uses different chemical-diffusive model than FAST and ALLA [208]. Reasonably good quantitative agreement between the theory and the literature data was observed for obstructed channel with height of $H=0.174 \mathrm{~m}$. 
(a)

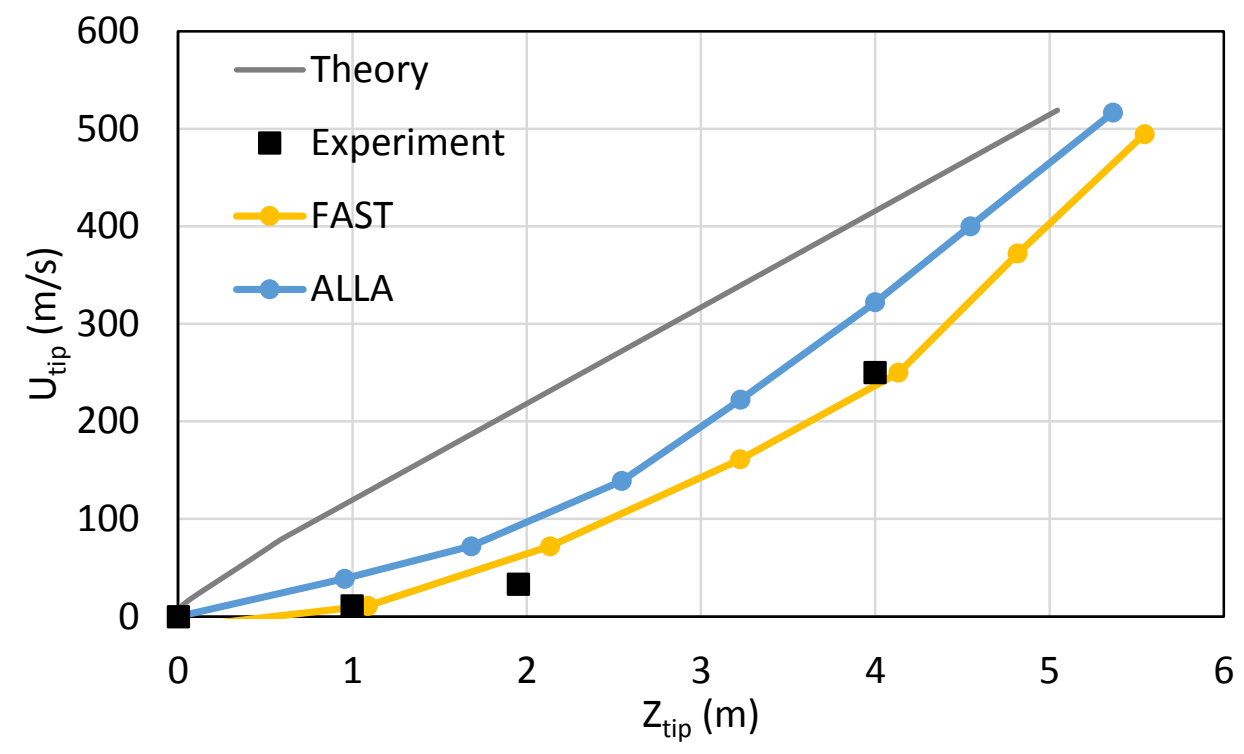

(b)

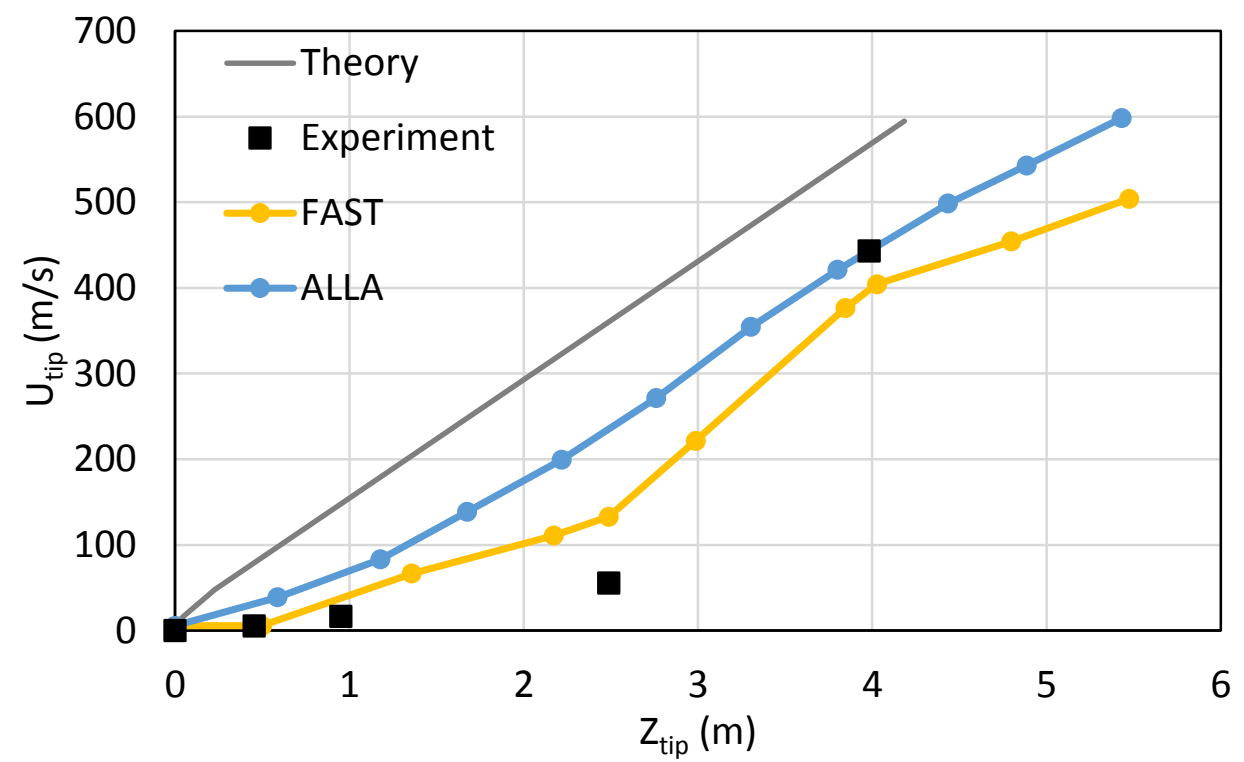

Figure 79: Comparison of theoretical formulation with the experiments [232] and numerical simulations [208] (FAST, yellow, \& ALLA, blue): the flame tip velocity vs its position for stoichiometric methane explosion in in obstructed channel with height $H=0.52 \mathrm{~m}$ and blockage ratios $\alpha=0.3$ (a) \& 0.6 (b).

Figures $79(\mathrm{a}, \mathrm{b})$ presents the counterparts of the Figs. $78(\mathrm{a}, \mathrm{b})$ for larger channel height $H=$ $0.52 \mathrm{~m}$ with the same blockage ratios of $\alpha=0.3$ and 0.6 . Theory and the literature data agree reasonably well. Slightly faster flame acceleration was observed in the theory comparing to FAST and ALLA simulations as well as to experiments. The linear trends in Figs. 78 and 79 represent exponential acceleration. 


\subsection{Comparison of Theory with Experiments and Simulations}

In all fire scenarios considered in this dissertation, finger flame acceleration mechanism was dealt with. First, in all conditions of the vented gas explosion experiments, flame propagated accordingly to this acceleration mechanism. For this reason, effect of boundary conditions that were assumed in studies dealing with finger flame acceleration mechanism was investigated. Finally, obstacles were added into geometry where its effect on finger flame acceleration was scrutinized. In all parts of this dissertation, flame studied accelerated in an exponential manner, typical in finger flame acceleration mechanism. It was also highlighted by Bychkov et al. [180] that finger flame acceleration is scale invariant. Therefore, acceleration rates will be a reasonable approach to compare the theoretical formulation with experiments and simulations conducted in the present dissertation.

If the propagation of the flame tip is assumed to follow the trend as $A \exp (\sigma t)$, where $A$ is constant and $t$ is time, $\sigma$ gives the exponential acceleration coefficient $(1 / s)$. These values were extracted from experiments, as presented in Sec. 4.1, and also from the simulations of Sec. 4.2. Specifically, Fig. 80 presents the comparison of the acceleration rate coefficients obtained from the experiments to that gotten from the theory (with $\alpha=0$, i.e. there is no obstruction to simulate the experiment condition) for fuel-lean $(\phi=0.8)$, stoichiometric $(\phi=1)$ and fuel-rich $(\phi=1.2)$ methane-air explosions in a single cylinder with center ignition. It was seen from the experiments that the highest acceleration rates were obtained in the stoichiometric mixtures. Theory predictions agree with this finding very well, except in the case of medium vent experiment where the theory predicts the highest acceleration rate in fuel-rich mixture. This is because of the longer duration of flame acceleration in this condition that results in higher acceleration rate than stoichiometric mixture. In other words, stoichiometric mixture reaches the end of the cylinder sooner than fuel-rich mixture because of faster flame acceleration. However, in theory, because the acceleration rate is time dependent due to the DL instability, longer time that it takes for fuel-rich mixture to reach the vent results in the higher acceleration rate coefficient. This is due to the fact that the longer the flame propagates the stronger the effect of DL instability is on the flame acceleration, at least in the theory. Overall, theory and experiments agree very well in the case of stoichiometric methane-air mixtures in all vent conditions. 


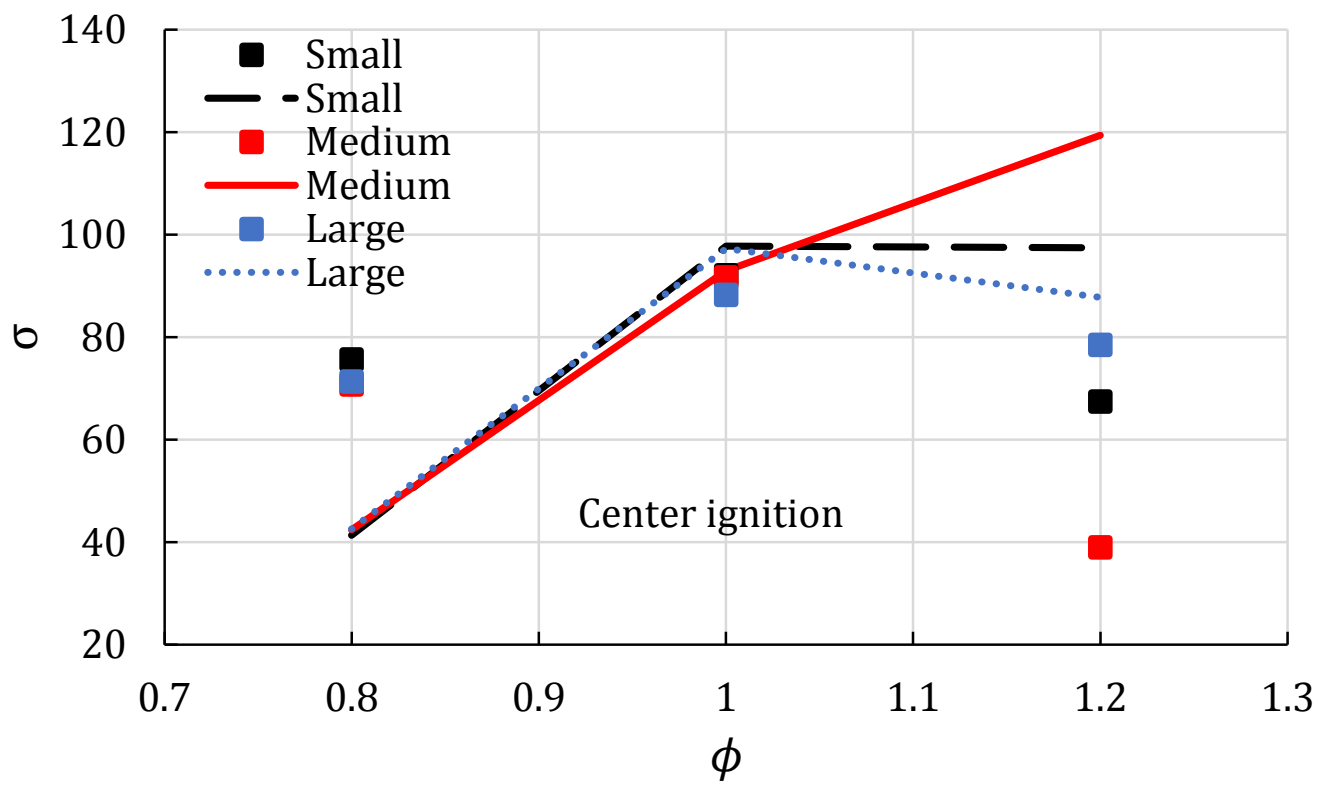

Figure 80: Comparison of acceleration rates obtained from the experiments (markers) and calculated from the theory (solid lines) for centrally-ignited fuel-lean, stoichiometric and fuel-rich methane-air explosions in a single cylinder with height of $H=19 \mathrm{~cm}$ and length of $L=30 \mathrm{~cm}$.

Figure 81 compares the acceleration rates obtained from rear-ignited methane explosions in extended cylinder $(60 \mathrm{~cm}$ in length) to that predicted by the theory. Qualitatively, the results of theory and experiments are in a good agreement. Both theory and experiments show that the highest acceleration rate happens when the mixture is stoichiometric and this is followed by the rich mixture. Quantitatively, the acceleration rate coefficient obtained from theory are higher than those obtained from the experiments in the cases of stoichiometric and fuel-rich methane explosions. Theory presumably over-predicts the DL instability in these conditions. For fuel-lean mixtures, both acceleration rate coefficients are in a reasonably good agreement with relative difference in the range of $9.7 \sim 24.6 \%$ for all vent areas.

Finally, the theory was compared to the simulations presented in Sec. 4.2. Specifically, the acceleration rate coefficients were extracted from the results of direct numerical simulations plotted versus scaled channel half-width along with their counterparts calculated by the theory (Fig. 82). The numerical simulation results are those with adiabatic and slip boundary conditions. Quantitatively, theory predicts higher acceleration rate coefficients than those of numerical simulations. The relative differences are $45.5 \%$ and $44.2 \%$ for $H / L_{f}=10$ and 20, respectively. This is because, the numerical simulation does not account for the DL instability, resulting in 
moderated flame acceleration. Qualitatively, numerical results showed that increased in the channel half-width did not cause an increase in the acceleration rate coefficient of the flame.

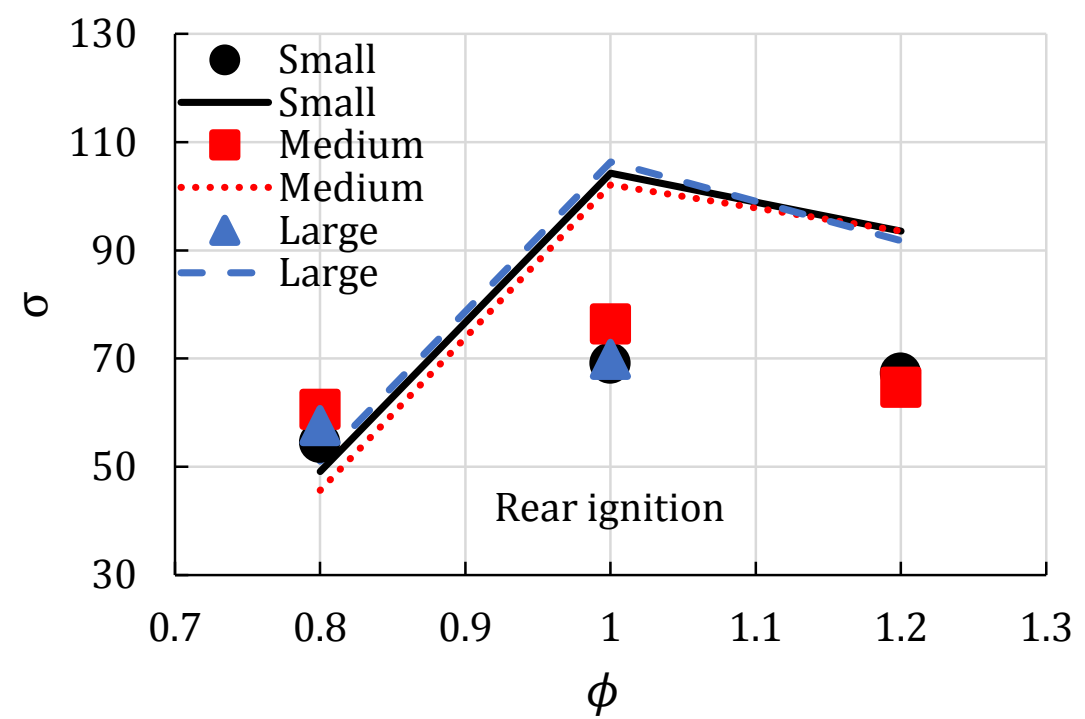

Figure 81: Comparison of acceleration rates obtained from the experiments (markers) and calculated from the theory (solid lines) for rear-ignited fuel-lean, stoichiometric and fuel-rich methane-air explosions in a single cylinder with height of $H=19 \mathrm{~cm}$ and length of $L=60 \mathrm{~cm}$.

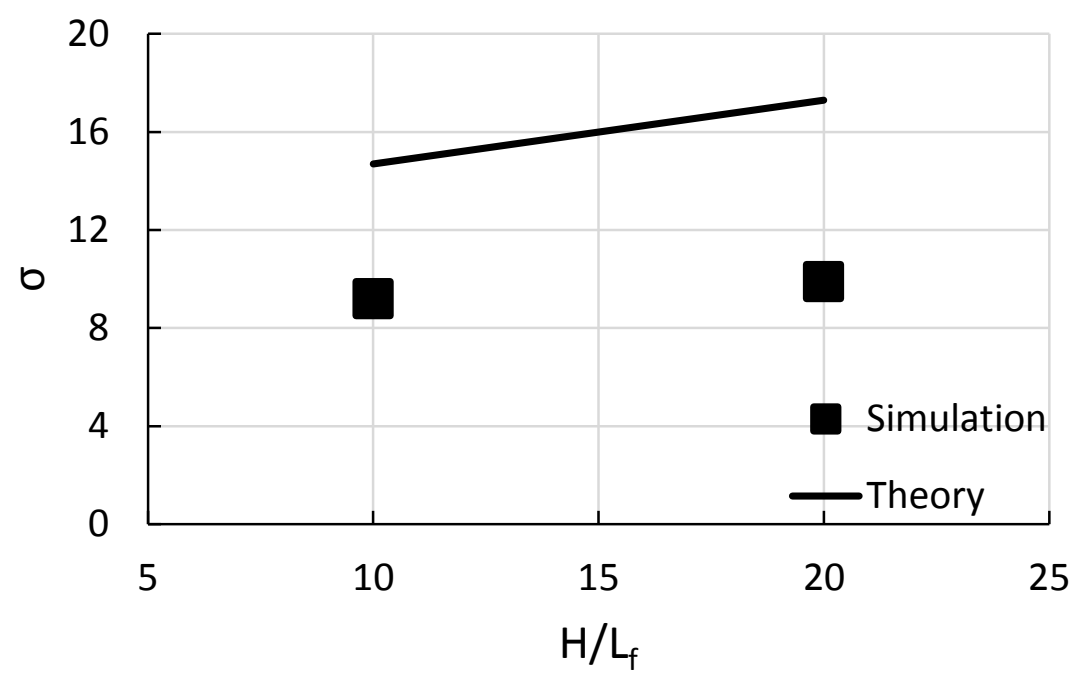

Figure 82: Comparison of acceleration rates obtained from the numerical simulations (markers) and calculated from the theory (solid lines) for rear-ignited methane-air explosions in a channel with various heights.

This certifies the validity of Bychkov's finding that the finger flame acceleration is scale-invariant. However, increase in the channel half-width resulted in, even though slightly, higher acceleration rate coefficient in theory's prediction. This is due to the DL instability effect. Larger the channels 
are; stronger the DL instability is in the theory. This finding goes against the statement that finger flame acceleration is scale-invariant due to the instability effect.

\section{Work in-progress}

\subsection{Dual-Chamber Compartment (Cylinder)}

A series of experiment were conducted in a dual-chamber compartment configuration. Specifically, two cylinders of length $30 \mathrm{~cm}$ were connected as shown in Fig. 83. In contrast to the extended cylinder configuration, considered before, here the second vent was placed at the end of first cylinder (in the middle of two-connected cylinders). Moreover, a second pressure transducer was mounted on the panel where the ignition occurs, Fig. 83. Here, the experiments were conducted using only rear ignition and medium $\left(86.6 \mathrm{~cm}^{2}\right)$ vent size. As before, the fuel-rich $(\phi=$ $1.2)$, stoichiometric $(\phi=1)$ and fuel-lean $(\phi=0.8)$ methane-air mixtures were used. A time shift between the pressure results of pressure transducers in the vent panel side and ignition location side was observed in the dual-chamber compartment experiments. Due to different time shifts in each experiment, pressure results of three repeated experiments for each three equivalence ratios were presented separately.

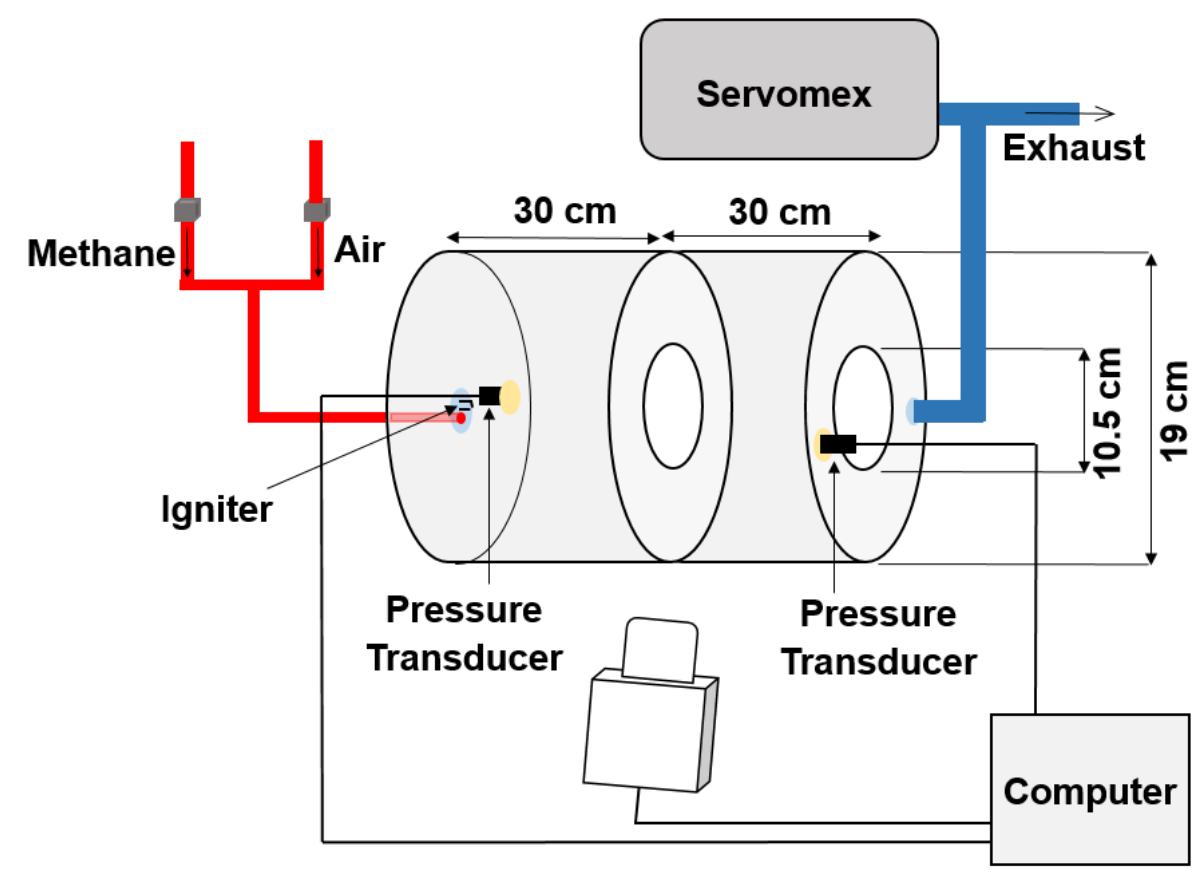

Figure 83: The experimental setup used for the dual-chamber compartment experiments. 

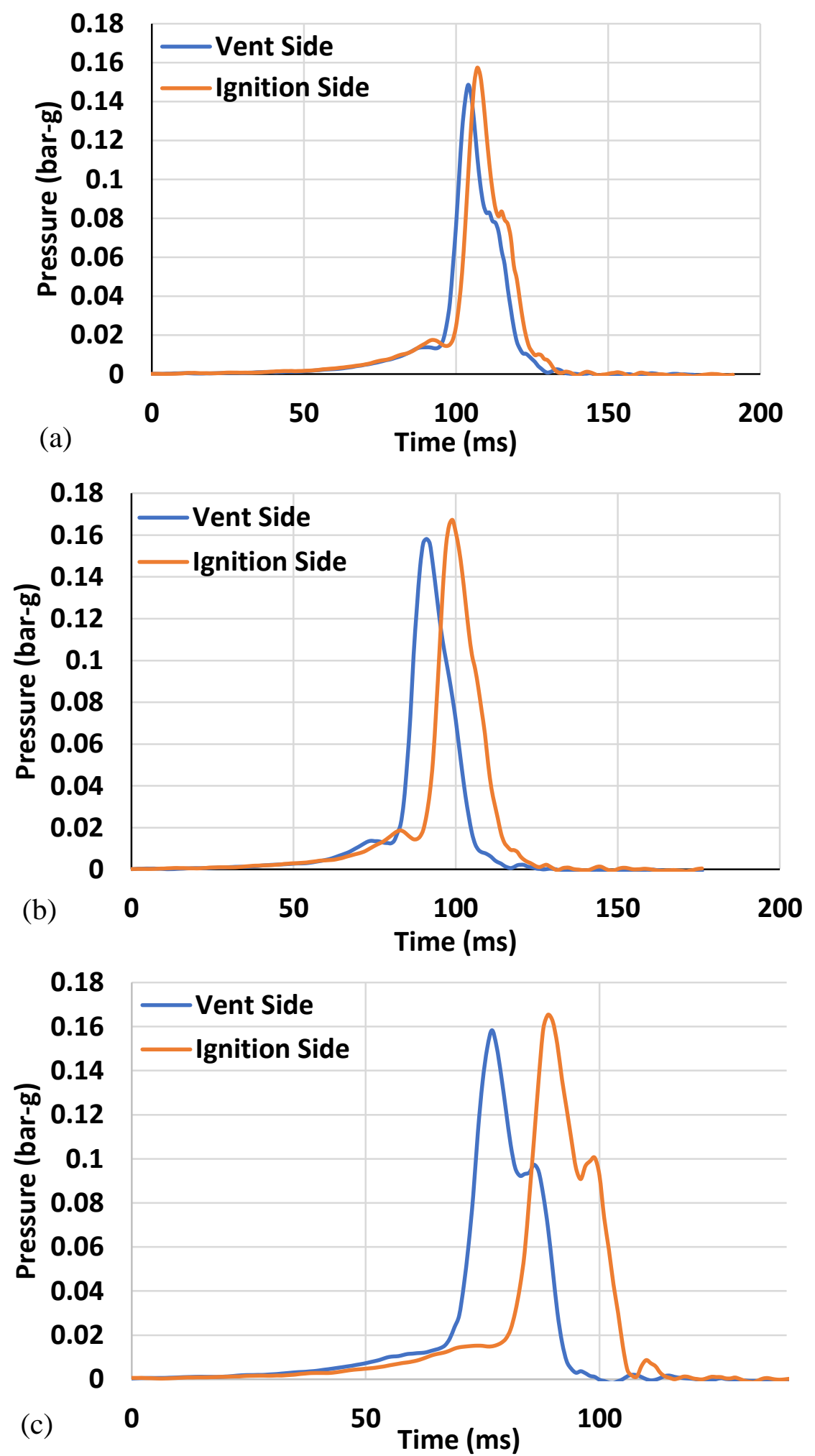

Figure 84: Pressure evolution for the lean $(\phi=0.8)$ methane-air explosions in a dual-chamber compartment cylinder with a medium $\left(86.6 \mathrm{~cm}^{2}\right)$ vent: a) Test 1, b) Test 2 , c) Test 3. 
Figure 84 presents the pressure evolution for the three repeated experiments for the lean $(\phi=0.8)$ methane-air mixture explosions in a dual-chamber compartment cylinder with a medium (86.6 $\mathrm{cm}^{2}$ ) vent and rear ignition. The orange lines show the pressure histories of the ignition location side and the blue lines show the pressure histories of the vent panel side. It is seen that the pressure transducer at the vent panel side read the pressure earlier than the pressure transducer at the ignition location side and this time shift was 1, 8 and $13 \mathrm{~ms}$ in Test 1, Fig. 84a, Test 2, Fig. 84b, and Test 3, Fig. $84 \mathrm{c}$, respectively. The average of maximum pressures $P_{\max }$ obtained in the three experiments was calculated as $P_{\max , a v g}=0.162$ bar-g at the ignition location side and $P_{\max , a v g}=$ 0.155 bar-g at the vent panel side.
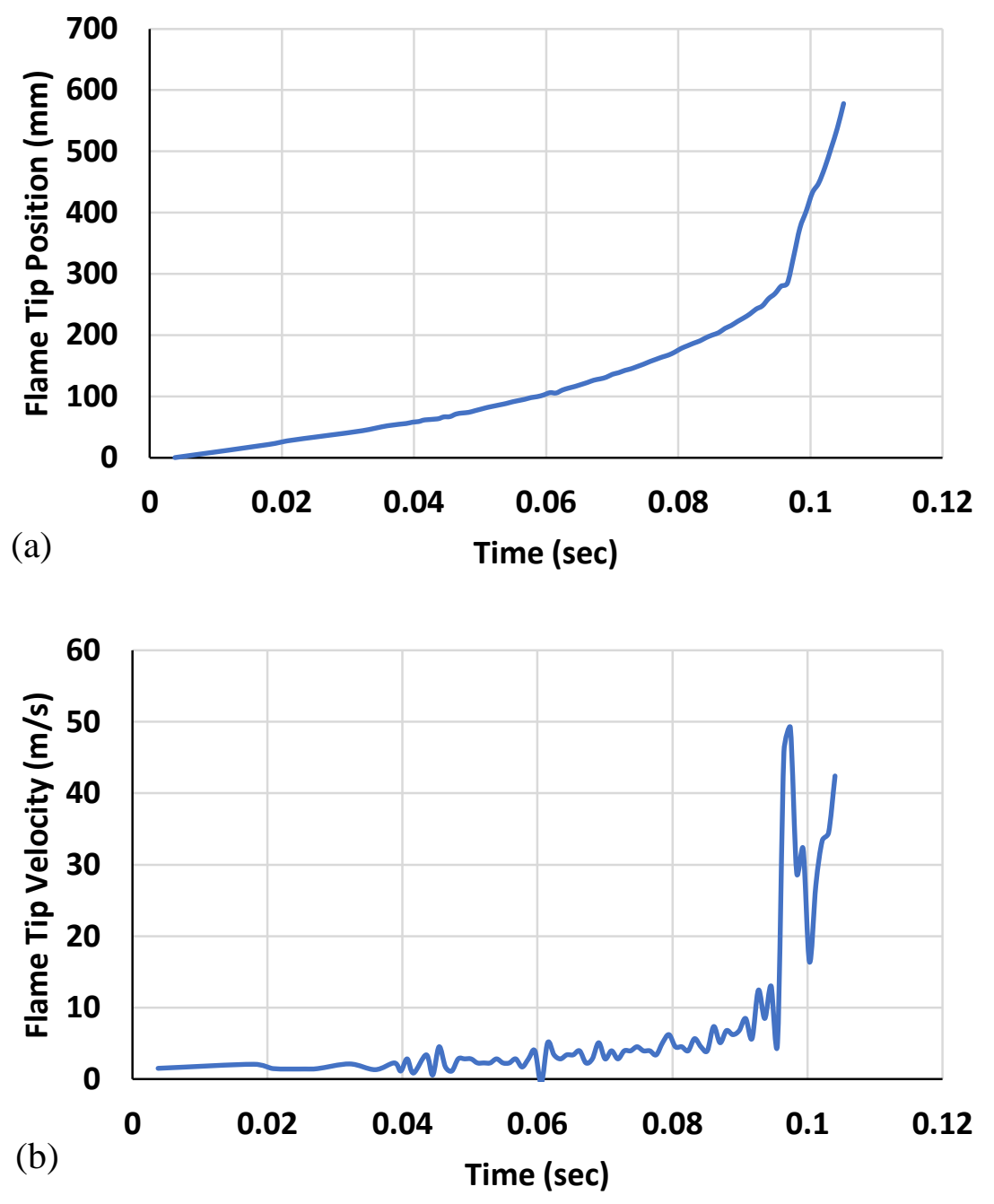

Figure 85: Evolutions of the flame tip position (a) and velocity (b) for the lean $(\phi=0.8)$ methane-air explosion in a dual-chamber compartment cylinder with medium $\left(86.6 \mathrm{~cm}^{2}\right)$ vent and rear ignition. 
(a)

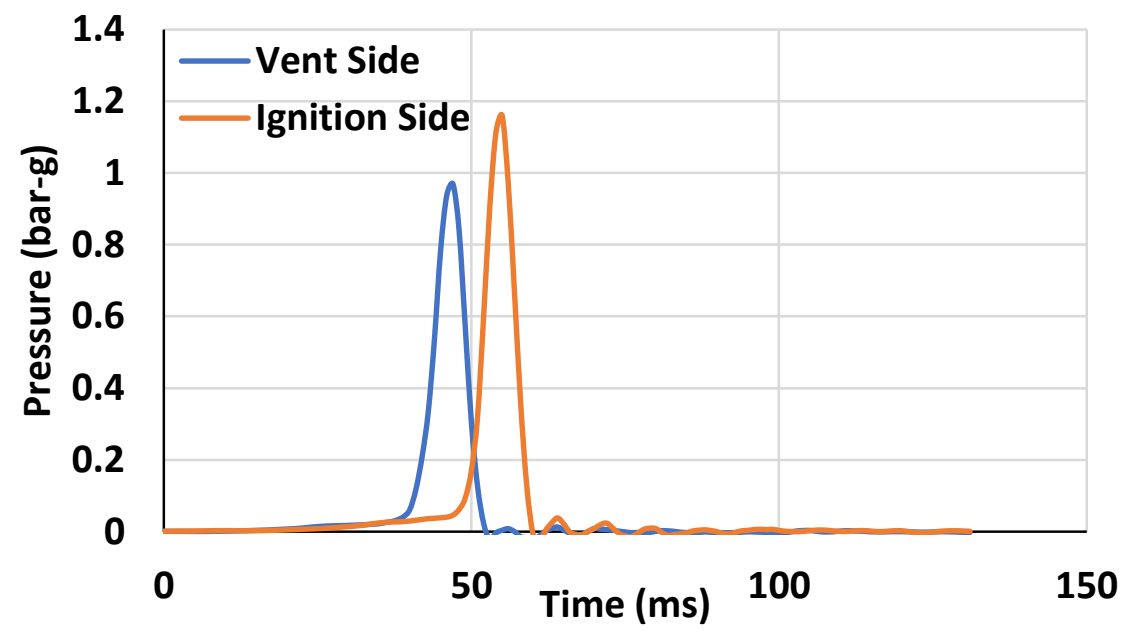

(b)
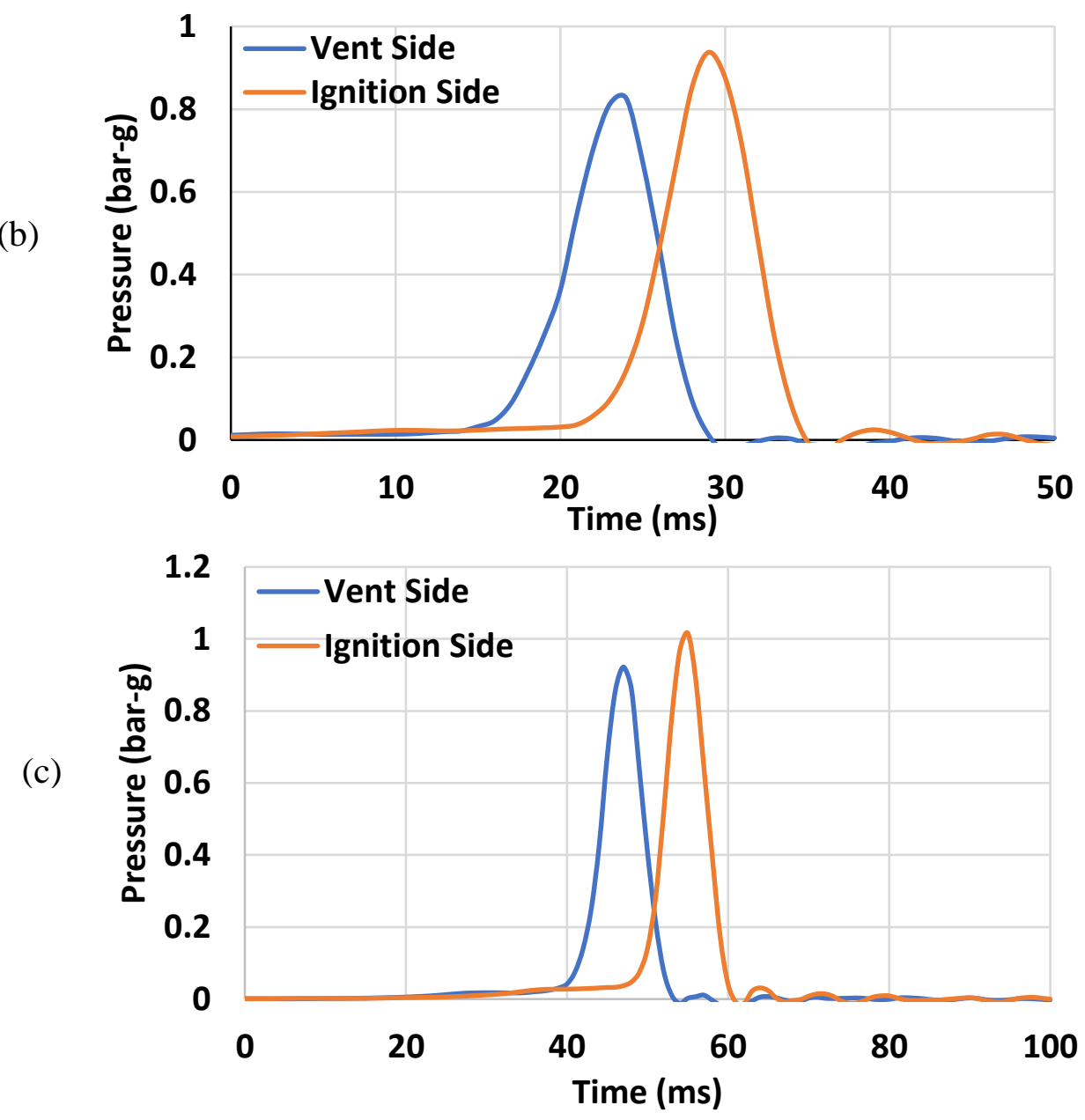

Figure 86: Pressure evolution for the stoichiometric $(\phi=1)$ methane-air explosions in a dual-chamber compartment cylinder with a medium $\left(86.6 \mathrm{~cm}^{2}\right)$ vent and rear ignition: a) Test 1, b) Test 2, c) Test 3 .

Figure 85 shows the evolutions of the flame tip position, Fig. 85a, and velocity, Fig. 85b, for the $\phi=0.8$ methane-air explosions in a dual-chamber compartment cylinder with a medium vent size 
$\left(86.6 \mathrm{~cm}^{2}\right)$ and rear ignition. It is seen that the flame experienced a jet flow at the second compartment with its velocity jumping to $U_{t i p}=49.2 \mathrm{~m} / \mathrm{s}$.

Figure 86 shows pressure evolution for three repeated experiments with stoichiometric methaneair explosions in the same dual-chamber compartment cylinder with a medium $\left(86.6 \mathrm{~cm}^{2}\right)$ vent area and rear ignition. It is again seen that the pressure transducer at the vent side read the pressure before the pressure transducer at the ignition side does. The time shifts between two pressure peaks were estimated as $8 \mathrm{~ms}$ for Test 1, Fig. 86a; $5 \mathrm{~ms}$ for Test 2, Fig. 86b; and, again, $8 \mathrm{~ms}$ for Test 3 , Fig. 86c. The average value of the maximum pressures over three experiments was $P_{\text {max }, \text { avg }}=$ 0.906 and 1.038 bar-g at the vent panel side and ignition location side, respectively.

(a)
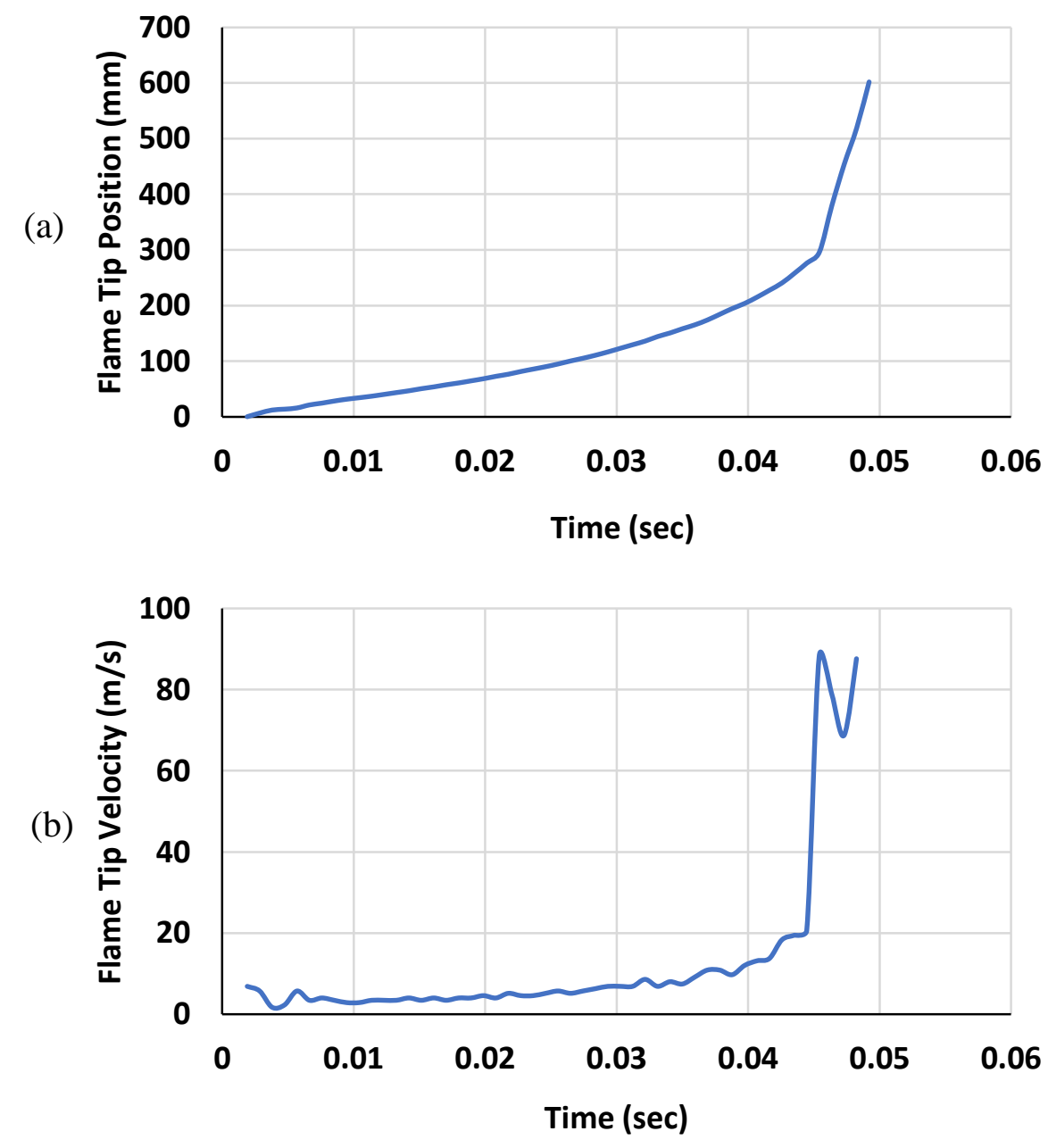

Figure 87: The evolutions of the flame tip position (a) and velocity (b) for stoichiometric $\phi=1$ methaneair explosions in dual-chamber compartment cylinder with medium $\left(86.6 \mathrm{~cm}^{2}\right)$ vent and rear ignition. 

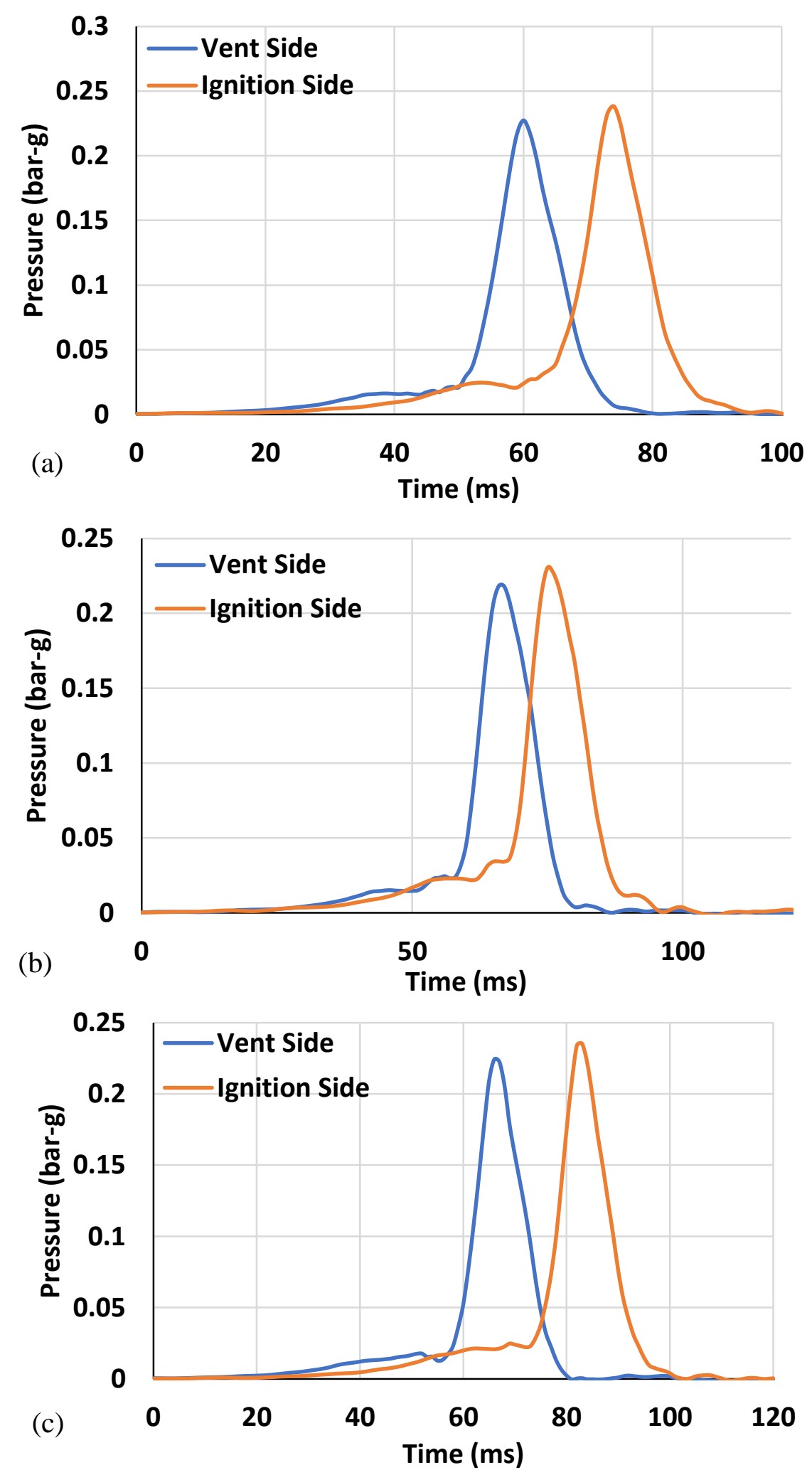

Figure 88: The pressure evolution of three repeated rich $(\phi=1.2)$ methane-air explosion experiments in dual-chamber compartment cylinder with medium $\left(86.6 \mathrm{~cm}^{2}\right)$ vent and rear ignition: a) Test 1 , b) Test 2, c) Test 3 . 
Figure 87 presents the evolutions of the flame tip position, Fig. 87a, and velocity, Fig. 87b, for stoichiometric methane-air explosions in a dual-chamber compartment cylinder having a medium $\left(86.6 \mathrm{~cm}^{2}\right)$ vent area and rear ignition. It is again seen that the flame velocity experienced a jump in the second compartment. This maximum flame tip velocity was calculated as $U_{t i p}=88.2 \mathrm{~m} / \mathrm{s}$.

Figure 88 shows the pressure evolution for the rich $(\phi=1.2)$ methane-air explosions in a dualchamber compartment cylinder with a medium $\left(86.6 \mathrm{~cm}^{2}\right)$ vent size and rear ignition. It can be seen that the pressure transducers at the vent panel side read the pressure before the pressure transducer at the ignition location side. The time shifts between the pressure peaks were estimated as $14 \mathrm{~ms}$ for Test 1, Fig. 88a; $8 \mathrm{~ms}$ for Test 2, Fig. 88b; and $17 \mathrm{~ms}$ for Test 3, Fig. 88c. The average values of maximum pressures were calculated as $P_{\max , a v g}=0.223$ and 0.234 bar-g for the pressure at the vent panel side and ignition location side, respectively.

(a)

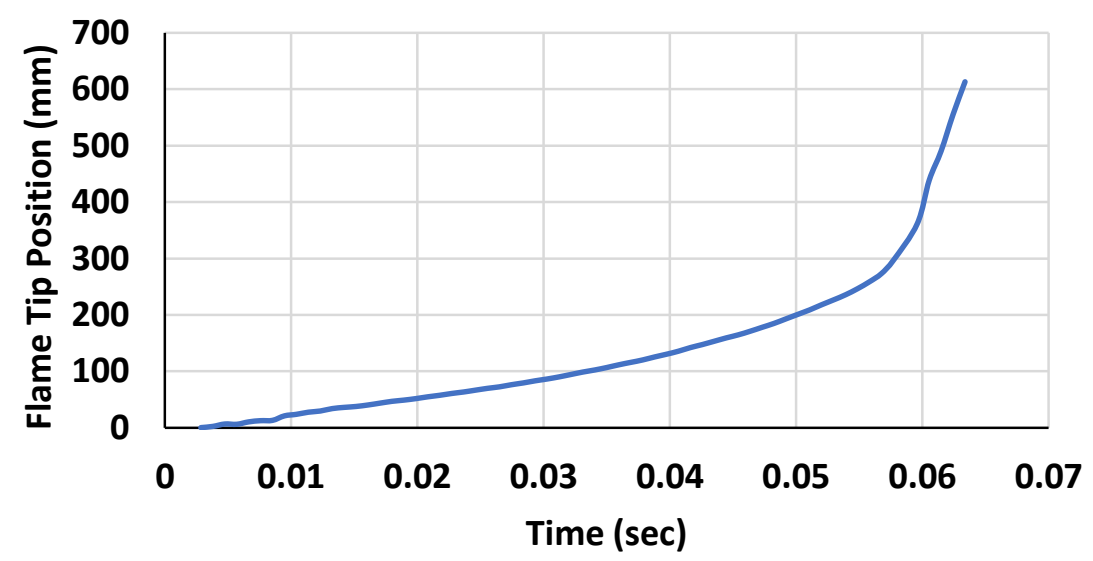

(b)

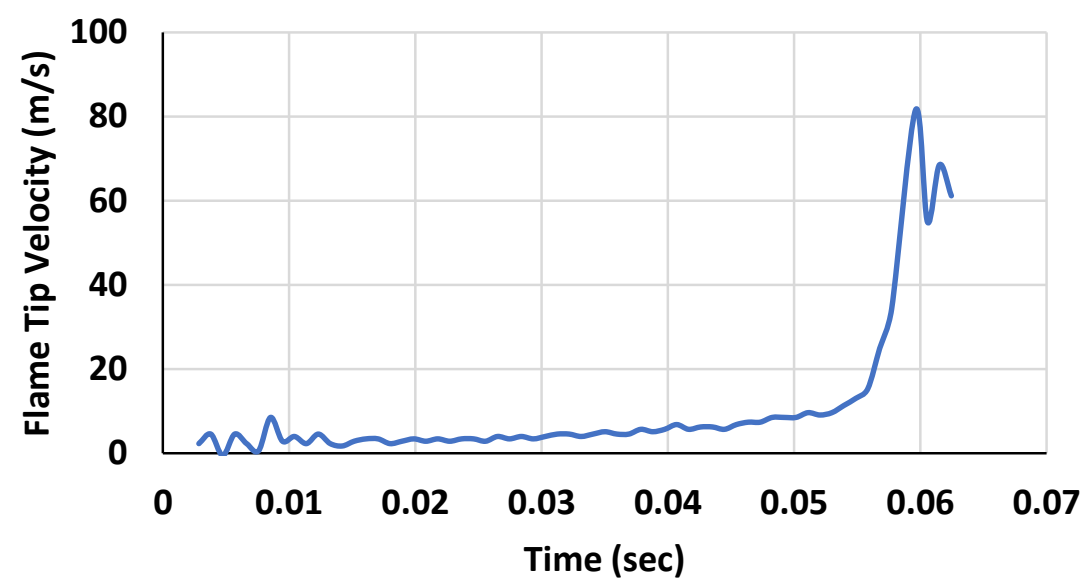

Figure 89: Evolutions of the flame tip position (a) and velocity (b) for the rich $(\phi=1.2)$ methane-air explosion in a dual-chamber compartment cylinder with a medium $\left(86.6 \mathrm{~cm}^{2}\right)$ vent area and rear ignition. 
Figure 89 presents the evolutions of the flame tip position, Fig. 89a, and velocity, Fig. 89b, for the rich $(\phi=1.2)$ methane-air explosions in a dual-chamber compartment cylinder with a medium $\left(86.6 \mathrm{~cm}^{2}\right)$ vent area and rear ignition. The flame tip position and velocity showed a jump when the flame front reached the second compartment. This was due to the contraction effect in the vent when the flame reached the first vent panel. The maximum flame tip velocity was $U_{\text {tip }}=81.5$ $\mathrm{m} / \mathrm{s}$. 


\section{Summary and Conclusions}

The objective of this dissertation was to establish an engineering model predicting the pressuretime histories of accidental gas explosions that occur in the process industries, with the aim that the minimum vent area could be predicted. For this reason, experiments were conducted in a smallscale volume and the pressure-time histories of experiments were compared to the predictions of the Explosion Venting Analyzer (EVA). In the experiments, it was observed that finger flame acceleration was the dominating acceleration mechanism, on which, many theoretical/numerical studies used assumptions for boundary conditions. The validity of such assumptions were verified by means of numerical simulations of fully-compressible reactive flow equations. A theoretical formulation was developed to investigate the effect of obstacles on the fire scenario in the smoothwalled passages, associated with finger flame acceleration. The new fire scenario was developed using the incompressible flow equations and was then extended to account for gas compressibility. Finally, the acceleration rates obtained from experiments, numerical simulations and analytical theory were compared.

The conclusions and outcome of this dissertation can be shown under four main categories.

\subsection{Experimental Study and Validation of EVA}

A series of experiments were performed in a transparent polycarbonate cylinder using the fuellean $\phi=0.8$, stoichiometric $\phi=1$ and fuel-rich $\phi=1.2$ methane-air mixtures. The mixtures were ignited at the center and rear of the cylinder, and three various vent areas were used to relieve the pressure; small $\left(67.9 \mathrm{~cm}^{2}\right)$, medium $\left(86.6 \mathrm{~cm}^{2}\right)$ and large $\left(132.7 \mathrm{~cm}^{2}\right)$. The vents were initially covered with aluminum foil and were cut in large portion prior to ignition to provide free-venting condition and avoid high pressure developments. A spark ignition was used to ignite the mixtures. The histories of the pressure and flame movements were recorded. Starting with a cylinder that has a length of $30 \mathrm{~cm}$, experiments were repeated for a twice longer cylinder. Additionally, preliminary results were presented for the dual-chamber experiments (Sec. 5). Moreover, an engineering model (EVA) that is based on theory and can predict the pressure-time histories of methane-air vented deflagrations in any enclosure sizes was updated. The model parameters were configured accordingly to experiments and the comparison of the experiments with the simulations were performed. The following conclusions were made: 
- The highest maximum pressure $P_{\max }$ was recorded in the stoichiometric $\phi=1$ methane-air mixtures in all cases.

- Center ignition resulted in lower peak pressures than rear ignition.

- The highest rate of pressure rise $(d P / d t)_{\max }$ was calculated in the stoichiometric $(\phi=1)$ methane-air explosions in all cases except when the vent area is large $\left(132.7 \mathrm{~cm}^{2}\right)$ and the mixture was ignited at the rear of the cylinder.

- The determination of the lowest maximum pressure and rate of pressure rise are not monotonic.

- In all experiments with rear ignition, the flame movement showed exponential acceleration for all equivalence ratios with highest acceleration rate obtained in the stoichiometric mixture.

- When the mixture was ignited centrally, the flame propagated toward two directions; one to the vent side and the other to the opposite wall side. It was seen that the flame towards the vent side accelerated in an exponential manner whereas the flame towards the wall side experienced a constant velocity movement.

- In the fuel-rich $\phi=1.2$ methane-air explosions and central ignition in the single cylinder, a flashback phenomenon was observed after the external burning when the flame spreads outside, the flame bounces back into the cylinder.

- In a cylinder with longer length but the same diameter resulted higher pressure build-up in the chamber and increased acceleration rate for the flame in all equivalence ratios.

- Overall, it was seen that the predictions of EVA for pressure rises as well as peak pressures of vented deflagrations were in a good agreement with what were observed in experiments.

\subsection{Numerical Study on Finger Flame Acceleration}

Numerical simulations of the reacting flow equations in $2 \mathrm{D}$ channels with fully-compressible hydrodynamics, transport properties (heat conduction, diffusion and viscosity) and single-step Arrhenius chemical kinetics were performed. The impacts of the shear-stress (slip/nonslip) and thermal (adiabatic/isothermal) wall conditions and different flow parameters such as the thermal expansion ratio, the wall temperature and the channel width on finger flame acceleration - an acceleration mechanism devoted to a situation, when a premixed flame front acquires a finger shape, after an expansion of an embryonic hemispherical flame were investigated [176,180,181]. The following findings were reported: 
- The surface boundary conditions provide only minor corrections during the early stages of burning, before a flame skirt contacts the wall.

- The effect of wall friction was observed as the flame approaches the sidewalls, where a distortion of the tulip flame occurs due to wall friction in the case of nonslip walls. After this stage, a flame front can propagate in the same manner as described by a classical Shelkin mechanism associated with wall friction at the nonslip walls [185].

- It is observed that the finger flame dynamics in adiabatic channels is similar to that in isothermal channels.

- A flame tends to accelerate faster when the wall temperature $T_{w}$ grows.

- The impact of $\Theta$ on acceleration is slightly higher in the case of the isothermal walls, and flames approach the sidewalls faster with lower $\Theta$.

\subsection{Analytical Study towards a Predictive Coal Mining Scenario and Validation with Experiments/Simulations from the Literature}

A step towards a predictive scenario of a flame propagation in obstructed passages was undertaken by developing a theoretical formulation, which combines the mechanism of finger flame acceleration [176, 180, 181] and ultrafast flame acceleration in obstructed pipes [206, 207] with that due to the DL instability [212]. Specifically, the 2D planar and cylindrical-axisymmetric geometries were considered and an incompressible flow assumption for various passage configurations and the compositions of the combustible premixture was employed. The newlyidentified flame propagation scenario has been studied in terms of the evolution of the flame tip position $Z_{t i p}$ and velocity (in the laboratory reference frame) $U_{t i p}$. Starting with homogeneouslygaseous combustion, the analysis has subsequently been extended to incorporate dust particles in the passage. Namely, inert and combustible dust as well as their combination are considered. Further, the effect of gas compressibility on predictive scenario of a burning accident in an obstructed 2D passage was investigated by accounting for a small but finite Mach number, associated with flame propagation, up to the first order. The following outcome was obtained:

- The role of the obstacles as well as the DL instability on a fire scenario is found to be significant, with a stronger effect observed in the cylindrical geometry.

- Closer to the stoichiometric condition, a flame propagates and accelerates faster; and acceleration is also promoted with an increase in the blockage ratio $\alpha$. 
- The combustible dust particles of radii 10-75 $\mu \mathrm{m}$ facilitate flame acceleration, while acceleration is moderated by the inert particles.

- The effect of particle size is significant in a way that smaller particles lead to faster flame acceleration.

- The effect of a type and size of the particles increases with the blockage ratio.

- Gas compression moderates flame acceleration noticeably as compared to the incompressible formulation. This is due to the modified flame parameters and additional nonlinear effect in the compressible formulation.

- The modified flame parameters seem to change the exponential trend of flame acceleration predicted by the incompressible theory, to a slower trend, whereas the nonlinear term in the evolution equation moderates, noticeably, the burning process at its later stage.

- The impact of the blockage ratio also weakens with gas compression.

- The analytical predictions of flame propagation were compared to the available experimental and numerical data in the literature. Results show reasonably good agreement.

\subsection{Comparison of Acceleration Rates Obtained in Finger Flame Accelerations}

In the experimental part of this work, it was realized that the finger flame acceleration was the driving flame propagation mechanism in the rear ignition experiments. Furthermore, the analytical theory developed in this dissertation simulates the finger flame acceleration mechanism when the blockage ratio is taken as zero. Thus, each part of this study dealt with finger flame acceleration and Bychkov et al. [180] highlighted that finger flame acceleration is scale-invariant, meaning its acceleration rate is same in micro-channels with that in subways and tunnels. For this reason, the acceleration rates obtained from experiments, simulations and analytical theory of this dissertation were compared. The results were qualitatively in good agreement. Quantitatively, in most of the comparisons, the acceleration rates predicted by the analytical theory were higher than those of experiments and simulations. The reason for this is the effect of the DL instability that provides additional acceleration to the flame.

Finally, this dissertation comprises of three main approaches; experimental, numerical and analytical approaches. The experimental part of this dissertation revealed the dynamics of methane-air flames in small-scale chambers with different vent sizes, and the outcome of this part was used to validate the EVA. Due to the limited time and resources, the investigation into larger 
scales were conducted by numerical and analytical approaches, whose outcomes are expected to be important in further development of EVA for larger scales. Specifically, the numerical approach investigated the effect of heat loss and wall friction on the flame dynamics in the unobstructed passages similar to the condition in experimental approach. This investigation filled the gap between the practical reality and studies in the literature that used adiabatic and slip wall conditions. Finally, a predictive fire scenario in an obstructed coalmining passage was investigated by means of analytical theory. The analytical predictions were in a reasonably good agreement with data available in the literature, proving its potential to be used in modelling the flame tip position and velocity in large-scale obstructed passages. 


\section{Future Work and Recommendations}

The outcome of this dissertation proves that EVA has potential in vented deflagration predictions and can be used in larger scales, such as subway, tunnels and coal mine. However, further research must be taken to justify the latter statement. Specifically, in this research, only small scale, relative to a coal mine for instance, experiments were conducted. It is known that in large scales, the flame acceleration may dominate over combustion process and change the burning scenario entirely. Furthermore, the experiments conducted in this dissertation dealt only with chamber volume that is empty. The presence of any equipment or congestion in the volume will definitely affect the flame dynamics and, subsequently, the severity of explosion. Therefore, work on volumes both with and without obstructions is recommended. Currently, the EVA is not capable of predicting pressure in gaseous-dusty environments for which additional research is recommended. Further research can also be conducted on multi-compartment arrangements that represent connected rooms in buildings or connected process equipment in an industry such as in nuclear or chemical plants. Preliminary work was conducted and shown in the Sec. 5. It is expected in this arrangement that the effect of flame dynamics will be of significant importance on the results of the event. For future work, to reveal more details of the relationship between the event and flame, it is recommended that synchronization of the ignition, pressure recording and camera is performed. Validation of the analytical theory developed here with the experiments and simulations certifies the potential use of the analytical theory in modeling the flame evolution for a solver in future work. 


\section{References}

[1] International Energy Agency. World energy outlook 2019, 2019.

[2] R.K. Eckhoff. Explosion hazards in the process industries. Elsevier/Gulf Professional Publishing, 2016.

[3] M.G. Zabetakis. Flammability characteristics of combustible gases and vapors. US Bureau of Mines, Bulletin 627, 1965.

[4] J.M. Kuchta. Investigation of fire and explosion accidents in the chemical, mining, and fuelrelated industries-a manual. US Department of the Interior, Bureau of Mines, Bulletin 680, 1985.

[5] M.A. Liberman. Introduction to physics and chemistry of combustion, explosion, flame, detonation. Springer, 2008.

[6] P. Clavin, G. Searby. Combustion waves and fronts in flows: flames, shocks, detonations, ablation fronts and explosion of stars. Cambridge University Press, 2016.

[7] A. Pekalski, J. Zevenbergen, S. Lemkowitz, H. Pasman. A review of explosion prevention and protection systems suitable as ultimate layer of protection in chemical process installations. Process Safety and Environmental Protection, 83 (1), 1-17, 2005.

[8] G.W. Jones, E.S. Harris, W.E. Miller. Explosive properties of acetone-air mixtures. US Department of Commerce, Bureau of Mines, Technical Paper 544, 1933.

[9] G.W. Jones, E.S. Harris, B.B. Beattie. Protection of equipment containing explosive acetoneair mixtures by the use of diaphragms. US Department of Commerce, Bureau of Mines, Technical Paper 553, 1933.

[10] T.S. Murphy. Determining needed relieving capacity for rupture diaphragms. Chemical and Metallurgical Engineering, 51 (12), 99-103, 1944.

[11] E.W. Cousins, P.E. Cotton. Design closed vessel to withstand internal explosions. Chemical Engineering, August, 133-137, 1951.

[12] J. Lamb. Explosions in enclosed crankcases of reciprocating engines: their cause, effect, and possible remedy. Proceedings of the Institution of Mechanical Engineers, 166, 327-349, 1952.

[13] J.H. Burgoyne, D.M. Newitt. Crankcase explosions in marine engines. Journal of American Society for Naval Engineers, 68, 122-128, 1955.

[14] H.G. Freeston, J.D. Roberts, A. Thomas. Crankcase explosions: an investigation into some factors governing the selection of protective devices. Proceedings of the Institution of Mechanical Engineers, 170, 811-824, 1956.

[15] W.P. Mansfield. Crankcase explosions: development of new protective devices. Proceedings of the Institution of Mechanical Engineers, 170, 825-862, 1956. 
[16] D.J. Rasbash, Z.W. Rogowski. The venting of gas and vapor explosions in duct systems. Part 1: Explosions of pentane vapor-air mixtures in a closed tube $6 \mathrm{ft}$ long x 6 in diameter. Fire Research Notes 257, 1956.

[17] D.J. Rasbash, Z.W. Rogowski. The venting of explosions in duct systems. Part II: The venting of explosions in a pentane-air mixture in a duct $6 \mathrm{ft}$ long x 6 in. diameter. Fire Research Notes 298, 1957.

[18] D.J. Rasbash, Z.W. Rogowski. Gaseous explosions in vented ducts. Combustion and Flame, 4, 301-312, 1960.

[19] D.J. Rasbash, Z.W. Rogowski. Relief of explosions in duct systems. Symposium on Chemical Process Hazards, 58-68, Manchester, England, 29-31 March, 1960.

[20] D.J. Rasbash, Z.W. Rogowski. The venting of gas and vapor explosions in duct systems. Part III: Vented explosions in straight unobstructed square ducts. Fire Research Notes 453, 1961.

[21] Joint Fire Research Organization. Symposium on flame arresters and relief vents held at joint fire research organization - November 1959. Fire Research Notes 441, 1960.

[22] D.J. Rasbash. Reliefs for gaseous and vapor explosions. Fire Research Notes 416, 1959.

[23] W.A. Simmonds, P.A. Cubbage. The design of explosion reliefs for industrial drying ovens. Symposium on Chemical Process Hazards, 69-77, Manchester, England, 29-31 March, 1960.

[24] J.H. Burgoyne, M.J.G. Wilson. The relief of pentane vapor-air explosions in vessels. Symposium on Chemical Process Hazards, 25-29, 1960.

[25] G. Munday. Rapid discharge of gases through rupture discs and similar pressure release mechanisms. Volume I. The University of London. PhD thesis, 1961.

[26] G. Munday. Rapid discharge of gases through rupture discs and similar pressure release mechanisms. Volume II. The University of London. PhD thesis, 1961.

[27] G. Munday. The calculation of venting areas for pressure relief of explosions in vessels. Second Symposium on Chemical Process Hazards, 46-54, Manchester, England, 2-4 April, 1963.

[28] Z.W. Rogowski, D.J. Rasbash. Relief of explosions in propane-air mixtures moving in a straight unobstructed duct. Second Symposium on Chemical Process Hazards, 21-28, Manchester, England, 2-4 April, 1963.

[29] K.N. Palmer, Z.W. Rogowski. The protection of equipment with flame arresters 1. Cubicle enclosures with commercial arresters. Fire Research Notes 613, 1966.

[30] K.N. Palmer, Z.W. Rogowski. The protection of equipment with flame arresters. 2. Effect of contents, and use of improved arresters. Fire Research Notes 658, 1967. 
[31] K.N. Palmer, Z.W. Rogowski. The use of flame arresters for protection of enclosed equipment in propane-air atmospheres. The Institution of Chemical Engineers Symposium Series, 25, 76-85, 1968.

[32] M. Charney. Explosive venting vs. explosion venting. Loss Prevention, 1, 35-38, 1967.

[33] G.F.P. Harris, P.G. Briscoe. The venting of pentane vapor-air explosions in a large vessel. Combustion and Flame, 11 (4), 329-338, 1967.

[34] H. Griffiths, S.A. Pugsley, S.O. Saunders. Report of the inquiry into the collapse of flats at Ronan Point, Canning Town. Ministry of Housing and Local Government. London Her Majesty's Stationery Office, 1968.

[35] D.J. Rasbash., D.J. The relief of gas and vapour explosions in domestic structures. Fire Research Notes 759, 1969.

[36] C. Yao, J. deRis, S.N. Bajpai, J.L. Buckley. Evaluation of protection from explosion overpressure in AEC gloveboxes. FM Research Corporation, 1969.

[37] C. Yao. Explosion venting of low-strength equipment and structures. Loss Prevention, 8, 19, 1974.

[38] The American Society of Mech. Engineers. ASME boiler and pressure vessel code. 1969.

[39] D.J. Rasbash, K.N. Palmer, Z.W. Rogowski, S.A. Ames. Gas explosions in multiple compartments. Fire Research Notes 847, 1970.

[40] D.A. Decker. Explosion venting guide. Fire Technology, 7, 219-223, 1971.

[41] G.E. Weldon. Damage limiting construction for chemical plants. Loss Prevention, 6, 105-111, 1972.

[42] E. Runes. Explosion venting. Loss Prevention, 6, 63-67, 1972.

[43] W.N. Howard. Interpretation of a building explosion accident, 6, 68-73, 1972.

[44] N.F. Astbury, H.W.H. West, H.R. Hodgkinson. Experimental gas explosions in load-bearing brick structures. National Bureau of Standards Special Publication 361 (1), 577-592, 1972.

[45] P.A. Cubbage, M.R. Marshall. Pressures generated in combustion chambers by the ignition of air-gas mixtures. The Institution of Chemical Engineers Symposium Series, 33, 24-31, 1972.

[46] M. Dragosavic. Structural measures against natural-gas explosions in high-rise blocks of flats. Heron, 19 (4), 1973.

[47] P.A. Cubbage, M.R. Marshall. Explosion relief protection for industrial plant of intermediate strength. Fifth International Symposium on Chemical Process Hazards, 196-210, 1974. 
[48] W.B. Howard, W.W. Russell. A procedure for designing gas combustion venting systems. Fifth Symposium on Chemical Process Hazard. The Institution of Chemical Engineers Symposium 39a, 179-195, Manchester, England, 9-11 April, 1974.

[49] R.N. Butlin, P.S. Tonkin. Pressures produced by gas explosions in a vented compartment. Fire Research Notes 1019, 1974.

[50] R.N. Butlin. A review of information on experiments concerning the venting of gas explosions in buildings. Fire Research Notes 1026, 1975.

[51] R.G. Zalosh. Explosion protection in refuse shredding. Proceedings of the Fifth National Congress, Waste Management Technology and Resource \& Energy Recovery, 76-107, 1976.

[52] M.J. Sapko, A.L. Furno, J.M. Kuchta. Flame and pressure development of large-scale $\mathrm{CH}_{4-}$ air- $\mathrm{N}_{2}$ Explosions. US Department of the Interior. Bureau of Mines Report of Investigations 8176, 1976.

[53] W.G. Chappell. Pressure/time diagram for explosion vented space. Loss Prevention, 11, 7686, 1977.

[54] W. Bartknecht. Explosion pressure relief. Loss Prevention, 11, 93-105, 1977.

[55] R.J. Harris, M.R. Marshall, D.J. Moppett. The response of glass windows to explosion pressures. The Institution of Chemical Engineers Symposium Series 49, 83-97, London, England, 5-7 April, 1977.

[56] Z.W. Rogowski. Explosion protection methods by reliefs. The Institution of Chemical Engineers Symposium Series 49, 63-71, London, England, 5-7 April, 1977.

[57] M.R. Marshall. Calculation of gas explosion relief requirements: the use of empirical equations. The Institution of Chemical Engineers Symposium Series 49, 21-28, London, England, 5-7 April, 1977.

[58] C. Donat. Pressure relief as used in explosion protection. Loss Prevention, 11, 87-92, 1977.

[59] E.J. Anthony. The use of venting formulae in the design and protection of building and industrial plant from damage by gas or vapour explosions. Journal of Hazardous Materials, 2, 23-49, $1977 / 78$.

[60] D. Bradley, A. Mitcheson. The venting of gaseous explosions in spherical vessels. I-theory. Combustion and Flame, 32, 221-236, 1978.

[61] D. Bradley, A. Mitcheson. The venting of gaseous explosions in spherical vessels. II-theory and experiment. Combustion and Flame, 32, 237-255, 1978.

[62] S. Crescitelli, G. Russo, V. Tufano. Analysis and design of venting systems: a simplified approach. Journal of Occupational Accidents, 2, 125-133, 1979.

[63] R.G. Zalosh. Gas explosion tests in room-size vented enclosures. Loss Prevention, 13, 98 $110,1979$. 
[64] I.G. Buckland. Explosion of gas layers in a room size chamber. The Institution of Chemical Engineers Symposium Series No. 58, 289-304, Manchester, England, 1-3 April, 1980.

[65] D.M. Solberg, J.A. Pappas, E. Skramstad. Observations of flame instabilities in large scale vented gas explosions. Proceedings of the Combustion Institute, 18 (1), 1607-1614, 1981.

[66] W. Bartknecht. Explosions, course, prevention, protection. Springer-Verlag, 1981.

[67] V. Tufano, S. Crescitelli, G. Russo. On the design of venting systems against gaseous explosions. Journal of Occupational Accidents, 3, 143-152, 1981.

[68] R.G. Zalosh, J.P. Coll. Explosion venting test program for municipal solid waste shredders. Society of Fire Protection Engineers Technology Report 81-9, 1981.

[69] S.R. Mulpuru, G.B. Wilkin. A model for vented deflagration of hydrogen in a volume. Atomic Energy of Canada Limited Research Company, 1982.

[70] M. Fairweather, M.W. Vasey. A mathematical model for the prediction of overpressures generated in totally confined and vented explosions. Proceedings of the Combustion Institute, 19 (1), 645-653, 1982.

[71] J.H.S. Lee, C.M. Guirao. Pressure development in closed and vented vessels. Plant/Operations Progress, 1 (2), 75-85, 1982.

[72] D.M. Solberg. Industrial gas explosion problems. Plant/Operations Progress, 1 (4), 243-248, 1982.

[73] W.B. Howard, A.H. Karabinis. Tests of explosion venting of buildings. Plant/Operations Progress, 1 (1), 51-65, 1982.

[74] V.V. Molkov, V.P. Nekrasov. Dynamics of gas combustion in a constant volume in the presence of exhaust. Combustion, Explosion, and Shock Waves, 18 (4), 363-369, 1982.

[75] C.J.M. Van Wingerden, J.P. Zeeuwen. On the role of acoustically driven flame instabilities in vented gas explosions and their elimination. Combustion and Flame, 51, 109-111, 1983.

[76] T. Hirano. Gas explosion processes in enclosures. Plant/Operations Progress, 3 (4), 247-254, 1984.

[77] I. Swift. Venting deflagrations-theory and practice. Plant/Operations Progress, 3 (2), 89-93, 1984.

[78] S. Chippett. Modeling of vented deflagrations. Combustion and Flame, 55, 127-140, 1984.

[79] D.P.J. McCann, G.O. Thomas, D.H. Edwards. Gasdynamics of vented explosions. Part I: experimental studies. Combustion and Flame, 59, 233-250, 1985.

[80] D.P.J. McCann, G.O. Thomas, D.H. Edwards. Gasdynamics of vented explosions. Part II: one-dimensional wave interaction model. Combustion and Flame, 60, 63-70, 1985. 
[81] M.G. Cooper, M. Fairweather, J.P. Tite. On the mechanisms of pressure generation in vented explosions. Combustion and Flame, 65, 1-14, 1986.

[82] W. Kordylewski, J. Wach. Influence of ducting on the explosion pressure. Combustion and Flame, 66, 77-79, 1986.

[83] W. Kordylewski, J. Wach. Influence of ducting on the explosion pressure: small scale experiments. Combustion and Flame, 71, 51-61, 1988.

[84] M. Epstein, I. Swift, H.K. Fauske. Estimation of peak pressure for sonic-vented hydrocarbon explosions in spherical vessels. Combustion and Flame, 66, 1-8, 1986.

[85] I. Swift, M. Epstein. Performance of low pressure explosion vents. Plant/Operations Progress, $6(2), 1987$.

[86] A.J. Harrison, J.A. Eyre. External explosions" as a result of explosion venting. Combustion Science and Technology, 52, 91-106, 1987.

[87] R.K. Kumar, T. Skraba, D.R. Greig. Vented combustion of hydrogen-air mixtures in large volumes. Nuclear Engineering and Design, 99, 305-315, 1987.

[88] R.K. Kumar, W.A. Dewit, D.R. Greig. Vented explosion of hydrogen-air mixtures in a large volume. Combustion Science and Technology, 66, 251-266, 1989.

[89] M. Epstein, G.M. Hauser, B.J. Tilley, C.H. Barron, J.L. Wise, P. Thistleton, R.L. Harper, M.J. Couture, B.R. Blair. A computer model for the estimation of peak pressure for sonic-vented tetrafluoroethylene decompositions. Journal of Loss Prevention in the Process Industries, 3, 370-380, 1990.

[90] F. Bouhard, B. Veyssiere, J.-C. Leyer, J. Chaineaux. Explosion in a vented vessel connected to a duct. AIAA Progress in Astronautics and Aeronautics, 134, 85-103, 1990.

[91] P. Canu, R. Rota, S. Carra. Vented gas deflagrations a detailed mathematical model tuned on a large set of experimental data. Combustion and Flame, 80, 49-64, 1990.

[92] R. Rota, P. Canu, S. Carra. Vented gas deflagration modeling: a simplified approach. Combustion and Flame, 85, 319-330, 1991.

[93] R. DeGood, K. Chatrathi. Comparative analysis of test work studying factors influencing pressures developed in vented deflagrations. Journal of Loss Prevention in the Process Industries, 4, 297-304, 1991.

[94] C.A. Catlin. Scale effects on the external combustion caused by venting of a confined explosion. Combustion and Flame, 83, 399-411, 1991.

[95] V.V. Molkov. Venting of gaseous explosions: turbulization aspect. Proceedings of the 1st Asian Conference on Fire Science, 480-484, 1992.

[96] V. Molkov, A. Baratov, A. Korolchenko. Dynamics of gas explosions in vented vessels: review and progress. AIAA Progress in Astronautics and Aeronautics, 154, 117-131, 1993. 
[97] E.A. Ural. A simplified method for predicting the effect of ducts connected to explosion vents. Journal of Loss Prevention in the Process Industries, 6 (1), 3-10, 1993.

[98] M.N. Carcassi, F. Fineschi. A theoretical and experimental study on the hydrogen vented deflagration. Nuclear Engineering and Design, 145, 355-364, 1993.

[99] W.P.M. Mercx, C.J.M. van Wingerden, H.J. Pasman. Venting of gaseous explosions. Process Safety Progress, 12 (1), 40-46, 1993.

[100] V.V. Molkov. Venting of deflagrations: dynamics of the process in systems with a duct and receiver. Fire Safety Science-Proceedings of the Fourth International Symposium, 12451254, Ottawa, Ontario Canada, 13-17 July, 1994.

[101] A. Alexiou, H. Phylaktou, G.E. Andrews. The effect of vent size on pressure generation in explosions in large L/D vessels. Combustion Science and Technology, 113-114, 645-652, 1996.

[102] R. Siwek. Explosion venting technology. Journal of Loss Prevention in the Process Industries, 9 (1), 81-90, 1996.

[103] R. Lautkaski. Understanding vented gas explosions. VTT Research Notes 1812, 1997.

[104] A. Alexiou, G.E. Andrews, H. Phylaktou. A comparison between end-vented and sidevented gas explosions in large L/D vessels. Process Safety and Environmental Protection, 75, 9-13, 1997.

[105] V. Molkov, A. Korolchenko, S. Alexandrov. Venting of deflagrations in buildings and equipment: universal correlation. Fire Safety Science-Proceedings of the Fifth International Symposium, 1249-1260, Melbourne, Australia, 3-7 March, 1997.

[106] V.V. Molkov. Venting of deflagrations: the dependence of turbulence factor on enclosure volume and vent ratio. Fire science and technology, Proceedings of the Third Asia-Oceania Symposium, 161-169, Singapore, 10-12 June, 1998.

[107] S. Cooper. Explosion venting-the predicted effects of inertia. The Institution of Chemical Engineers Symposium Series, 144, 305-319, Manchester, UK, 10-12 November, 1998.

[108] S. Hochst, W. Leuckel. On the effect of venting large vessels with mass inert panels. Journal of Loss Prevention in the Process Industries, 11, 89-97, 1998.

[109] V.V. Molkov. Explosions in buildings: modeling and interpretation of real accidents. Fire Safety Journal, 33, 45-56, 1999.

[110] B. Ponizy, J.C. Leyer. Flame dynamics in a vented vessel connected to a duct: 1. mechanism of vessel-duct interaction. Combustion and Flame, 116, 259-271, 1999.

[111] B. Ponizy, J.C. Leyer. Flame dynamics in a vented vessel connected to a duct: 2 . influence of ignition site, membrane rupture, and turbulence. Combustion and Flame, 116, 272-281, 1999. 
[112] V. Molkov, R. Dobashi, M. Suzuki, T. Hirano. Modeling of vented hydrogen-air deflagrations and correlations for vent sizing. Journal of Loss Prevention in the Process Industries, 12, 147-156, 1999.

[113] B. Ponizy, B. Veyssiere. Mitigation of explosions in a vented vessel connected to a duct. Combustion Science and Technology, 158, 167-182, 2000.

[114] S.K. Chow, R.P. Cleaver, M. Fairweather, D.G. Walker. An experimental study of vented explosions in a 3:1 aspect ratio cylindrical vessel. Process Safety and Environmental Protection, 78, 425-433, 2000.

[115] T. Forcier, R. Zalosh. External pressures generated by vented gas and dust explosions. Journal of Loss Prevention in the Process Industries, 13, 411-417, 2000.

[116] V. Molkov, R. Dobashi, M. Suzuki, T. Hirano. Venting of deflagrations: hydrocarbon-air and hydrogen-air systems, Journal of Loss Prevention in the Process Industries, 13, 397409, 2000.

[117] D.M. Razus, U. Krause. Comparison of empirical and semi-empirical calculation methods for venting of gas explosions. Fire Safety Journal, 36, 1-23, 2001.

[118] V.V. Molkov. Unified correlations for vent sizing of enclosures at atmospheric and elevated pressures. Journal of Loss Prevention in the Process Industries, 14, 567-574, 2001.

[119] F. Tamanini. Scaling parameters for vented gas and dust explosions. Journal of Loss Prevention in the Process Industries, 14, 455-461, 2001.

[120] V.V. Molkov, R.M. Eber, A.V. Grigorash, F. Tamanini, R. Dobashi. Vented gaseous deflagrations: modelling of translating inertial vent covers. Journal of Loss Prevention in the Process Industries, 16, 395-402, 2003.

[121] V.V. Molkov, A.V. Grigorash, R.M. Eber, D.V. Makarov. Vented gaseous deflagrations: modelling of hinged inertial vent covers. Journal of Hazardous Materials, A116, 1-10, 2004.

[122] V.V. Molkov, A.V. Grigorash, R.M. Eber. Vented gaseous deflagrations: Modelling of spring-loaded inertial vent covers. Fire Safety Journal, 40, 307-319, 2005.

[123] V.V. Molkov, A.V. Grigorash, R.M. Eber, F. Tamanini, R.Dobashi. Vented gaseous deflagrations with inertial vent covers: state-of-the-art and progress. Process Safety Progress, 23 (1), 29-36, 2004.

[124] G. Ferrara, S.K. Willacy, H.N. Phylaktou, G.E. Andrews, A. Di Benedetto, M.C. Mkpadi. Duct-vented propane/air explosions with central and rear ignition. Fire Safety ScienceProceedings of the Eighth International Symposium, 1341-1352, Beijing, China, 18-23 September, 2005.

[125] X. Jiang, B. Fan, J. Ye, G. Dong. Experimental investigations on the external pressure during venting. Journal of Loss Prevention in the Process Industries, 18, 21-26, 2005. 
[126] J. Hu, Y. Pu, F. Jia. Study of gas combustion in a vented cylindrical vessel. Combustion Science and Technology, 177, 323-346, 2005.

[127] Z. Chen, B. Fan, X. Jiang, J. Ye. Investigations of secondary explosions induced by venting. Process Safety Progress, 25 (3), 255-261, 2006.

[128] R.K. Kumar. Vented combustion of hydrogen-air mixtures in a large rectangular volume. 44th AIAA Aerospace Sciences Meeting and Exhibit, Reno, Nevada USA, 9-12 January, 2006.

[129] G. Ferrara, A. Di Benedetto, E. Salzano, G. Russo. CFD analysis of gas explosions vented through relief pipes. Journal of Hazardous Materials, A137, 654-665, 2006.

[130] Z. Du, X. Jin, D. Cui, J. Ye. The investigation of correlated factors of external explosion during the venting process. Journal of Loss Prevention in the Process Industries, 19, 326333, 2006.

[131] B. Janovsky, P. Selesovsky, J. Horkel, L. Vejsa. Vented confined explosions in Stramberk experimental mine and AutoReaGas simulation. Journal of Loss Prevention in the Process Industries, 19, 280-287, 2006.

[132] European Committee for Standardization. European standard EN 1991-1-7. 2006.

[133] V. Molkov, D. Makarov, J. Puttock. The nature and large eddy simulation of coherent deflagrations in a vented enclosure-atmosphere system. Journal of Loss Prevention in the Process Industries, 19, 121-129, 2006.

[134] S.K. Willacy, H.N. Phylaktou, G.E. Andrews, G. Ferrara. Stratified propane-air explosions in a duct vented geometry effect of concentration, ignition and injection position. Process Safety and Environmental Protection, 85, 153-161, 2007.

[135] P. Russo, A. Di Benedetto. Effects of a duct on the venting of explosions-critical review. Process Safety and Environmental Protection, 85, 9-22, 2007.

[136] V. Molkov, D. Makarov, F. Verbecke, Z. Mansurov, M. Zhumabaev. LES model of vented explosion: hydrogen-air mixtures. Proceedings of the 5th International Seminar on Fire and Explosion Hazards, 187-196, 2007.

[137] J. Karnesky, P. Chatterjee, F. Tamanini, S. Dorofeev. An application of 3D gasdynamic modeling for the prediction of overpressures in vented enclosures. Journal of Loss Prevention in the Process Industries, 20, 447-454, 2007.

[138] R.M. Kasmani, G.E. Andrews, H.N. Phylaktou, S.K. Willacy. Influence of static burst pressure and ignition position on duct-vented gas explosions. Proceedings of the 5th International Seminar on Fire and Explosion Hazards, 254-264, 2007.

[139] R.M. Kasmani, G.E. Andrews, H.N. Phylaktou. Experimental study on vented gas explosion in a cylindrical vessel with a vent duct. Process Safety and Environmental Protection, 91, 245-252, 2013. 
[140] British Standards. Gas explosion venting protective systems. BS EN 14994:2007. 2007.

[141] S. Willacy. Homogeneous and stratified vented gas explosions. University of Leeds. PhD thesis. 2008.

[142] G. Ferrara, S.K. Willacy, H.N. Phylaktou, G.E. Andrews, A. Di Benedetto, E. Salzano, G. Russo. Venting of gas explosion through relief ducts: interaction between internal and external explosions. Journal of Hazardous Materials, 155, 358-368, 2008.

[143] R.M. Kasmani. Vented gas explosions. University of Leeds. PhD thesis. 2008.

[144] R. Zalosh. Explosion venting data and modeling research project. The Fire Protection Research Foundation. 2008.

[145] C.R. Bauwens, J. Chaffee, S. Dorofeev. Experimental and numerical study of methane-air deflagrations in a vented enclosure. Fire Safety Science-Proceedings of the Ninth International Symposium, 1043-1054, Karlsruhe, Germany, 21-26 September, 2008.

[146] C. Proust, E. Leprette. The dynamics of vented gas explosions. Process Safety Progress, 29 (3), 231-235, 2009.

[147] C.R. Bauwens, J. Chaffee, S. Dorofeev. Effect of instabilities and acoustics on pressure generated in vented propane-air explosions. $22^{\text {nd }}$ International Colloquium on the Dynamics of Explosions and Reactive Systems, 2009.

[148] R.M. Kasmani, G.E. Andrews, H.N. Phylaktou. The influence of vessel volume and equivalence ratio of hydrocarbon/air mixtures in vented gas explosions. 4th International Conference on Safety \& Environment in Process Industry. Chemical Engineering Transactions, 463-468, Florence, Italy, 14-17 March, 2010.

[149] R.M. Kasmani, G.E. Andrews, H.N. Phylaktou, N. Ibrahim, R.R. Ali. Experimental investigation of vessel volume and equivalence ratio in vented gas. International Journal of Chemical, Molecular, Nuclear, Materials and Metallurgical Engineering, 6 (10), 932-936, 2012.

[150] C.R. Bauwens, J. Chaffee, S. Dorofeev. Effect of ignition location, vent size, and obstacles on vented explosion overpressures in propane-air mixtures. Combustion Science and Technology, 182, 1915-1932, 2010.

[151] B.J. Lowesmith, C. Mumby, G. Hankinson, J.S. Puttock. Vented confined explosions involving methane/hydrogen mixtures. International Journal of Hydrogen Energy, 36, 23372343, 2011.

[152] B.M. Fakandu, R.M. Kasmani, G.E. Andrews, H.N. Phylaktou. Explosion venting and mixture reactivity influences in a small vessel. $23^{\text {rd }}$ International Colloquium on the Dynamics of Explosions and Reactive Systems, Irvine, California USA, 24-29 July, 2011. 
[153] C.R. Bauwens, J. Chaffee, S.B. Dorofeev. Vented explosion overpressures from combustion of hydrogen and hydrocarbon mixtures. International Journal of Hydrogen Energy, 36, 2329-2336, 2011.

[154] J. Chow, C.R. Bauwens, S.B. Dorofeev. An analysis of peak overpressures in vented gaseous explosions. Proceedings of the Combustion Institute, 33, 2367-2374, 2011.

[155] B. Fakandu, R.M. Kasmani, G.E. Andrews, H.N. Phylaktou. The venting of hydrogen-air explosions in an enclosure with $\mathrm{L} / \mathrm{D}=2.8$. Proceedings of the Ninth International Symposium on Hazardous Process Materials and Industrial Explosions (IX ISHPMIE), Crakow, Poland, 22-27 July, 2012.

[156] C.R. Bauwens, J. Chao, S.B. Dorofeev. Effect of hydrogen concentration on vented explosion overpressures from lean hydrogen-air deflagrations. International Journal of Hydrogen Energy, 37, 17599-17605, 2012.

[157] B.M. Fakandu, Z.X. Yan, H.N. Phylaktou, G.E. Andrews. The effect of vent area distribution in gas explosion venting and turbulent length scale influence on the external explosion overpressure. Proceedings of the Seventh International Seminar on Fire \& Explosion Hazards (ISFEH7), Providence, Rhode Island USA, 5-10 May, 2013.

[158] J. Sustek, B. Janovsky. Comparison of empirical and semi-empirical equations for vented gas explosion with experimental data. Journal of Loss Prevention in the Process Industries, 26, 1549-1557, 2013.

[159] J. Daubech, C. Proust, O. Gentilhomme, C. Jamois, L. Mathieu. Hydrogen-air vented explosions: new experimental data. 5th International Conference on Hydrogen Safety, Brussels, Belgium, 9-11 September, 2013.

[160] B.M. Fakandu, G.E. Andrews, H.N. Phylaktou. Impact of non-central vents on vented explosion overpressures. X International Symposium on Hazardous Process Materials and Industrial Explosions (ISHPMIE), 2014.

[161] B.M. Fakandu. Vented gas explosions. The University of Leeds. PhD thesis, 2014.

[162] M. Schiavetti, A. Marangon, M. Carcassi. Experimental study of vented hydrogen deflagration with ignition inside and outside the vented volume. International Journal of Hydrogen Energy, 39, 20455-20461, 2014.

[163] X. Rocourt, S. Awamat, I. Sochet, S. Jallais. Vented hydrogen-air deflagration in a small enclosed volume. International Journal of Hydrogen Energy, 39, 20462-20466, 2014.

[164] J. Guo, X. Sun, S. Rui, Y. Cao, K. Hu, C. Wang. Effect of ignition position on vented hydrogen-air explosions. International Journal of Hydrogen Energy, 40, 15780-15788, 2015.

[165] F. Hernandez, M. Abdel-jawad, H. Hao. Simplified multiple equations' inverse problem of vented vessels subjected to internal gas explosions. Journal of Loss Prevention in the Process Industries, 35, 65-79, 2015. 
[166] G. Tomlin, D.M. Johnson, P. Cronin, H.N. Phylaktou, G.E. Andrews. The effect of vent size and congestion in large-scale vented natural gas/air explosions. Journal of Loss Prevention in the Process Industries, 35, 169-181, 2015.

[167] B.M. Fakandu, G.E. Andrews, H.N. Phylaktou. Vent burst pressure effects on vented gas explosion reduced pressure. Journal of Loss Prevention in the Process Industries, 36, 429438, 2015.

[168] J. Guo, Q. Li, D. Chen, K. Hu, K. Shao, C. Guo, C. Wang. Effect of burst pressure on vented hydrogen-air explosion in a cylindrical vessel. International Journal of Hydrogen Energy, 40, 6478-6486, 2015.

[169] J. Guo, C. Wang, Q. Li, D. Chen. Effect of the vent burst pressure on explosion venting of rich methane-air mixtures in a cylindrical vessel. Journal of Loss Prevention in the Process Industries, 40, 82-88, 2016.

[170] S. Qi, Y. Du, S. Wang, Y. Zhou, G. Li. The effect of vent size and concentration in vented gasoline-air explosions. Journal of Loss Prevention in the Process Industries, 44, 88-94, 2016.

[171] O.J. Ugarte, V. Akkerman, A.S. Rangwala. A computational platform for gas explosion venting. Process Safety and Environmental Protection, 99, 167-174, 2016.

[172] H. Sezer, F. Kronz, V. Akkerman, A.S. Rangwala. Methane-induced explosions in vented enclosures. Journal of Loss Prevention in the Process Industries, 48, 199-206, 2017.

[173] National Fire Protection Association. NFPA 68 standard on explosion protection by deflagration venting, 2018 edition. 2018.

[174] D.P. Nolan. Handbook of fire and explosion protection engineering principles. William Andrew/Elsevier, 2014.

[175] G. Ciccarelli, S. Dorofeev. Flame acceleration and transition to detonation in ducts. Progress in Energy and Combustion Science, 34 (4), 499-550, 2008.

[176] C. Clanet, G. Searby. On the 'tulip flame' phenomenon. Combustion and Flame, 105 (1-2), 225-238, 1996.

[177] K.I. Schelkin. The impact of the tube wall roughness on the emergence and propagaiton of a detonation in gases, Zh. Eksp. Teor. Fiz., 10, 823-827, 1940.

[178] O. Ugarte, V. Bychkov, J. Sadek, D. Valiev, V. Akkerman. Critical role of blockage ratio for flame acceleration in channels with tightly spaced obstacles. Physics of Fluids, 28 (9), $093602,2016$.

[179] O. Ellis. Flame movement in gaseous explosive mixtures. Fuel in Science and Practice, VII, 502-508, 1928.

[180] V. Bychkov, V. Akkerman, G. Fru, A. Petchenko, L.-E. Eriksson. Flame acceleration in the early stages of burning in tubes. Combustion and Flame, 150 (4), 263-276, 2007. 
[181] D. Valiev, V. Akkerman, M. Kuznetsov, L.-E. Eriksson, C.K. Law, V. Bychkov. Influence of gas compression on flame acceleration in the early stage of burning in tubes. Combustion and Flame, 160 (1), 97-111, 2013.

[182] S. Demir, V. Bychkov, S.H.R. Chalagalla, V. Akkerman. Towards a predictive scenario of a burning accident in a mining passage. Combustion Theory and Modelling, 21 (6), 9971022, 2017.

[183] S. Demir, A.R. Calavay, V. Akkerman. Influence of gas compression on a burning accident in a mining passage. Combustion Theory and Modelling, 22 (2), 338-358, 2018.

[184] H. Xiao, R. Houim, E. Oran. Formation and evolution of distorted tulip flames. Combustion and Flame, 162 (11), 4084-4101, 2015.

[185] V. Bychkov, A. Petchenko, V. Akkerman. L.-E. Eriksson. Theory and modeling of accelerating flames in tubes. Physical Review E, 72 (4), 046307, 2005.

[186] W. Han, Y. Gao, C.K. Law. Flame acceleration and deflagration-to-detonation transition in micro- and macro-channels: An integrated mechanistic study. Combustion and Flame, 176, 285-298, 2017.

[187] M.F. Ivanov, A.D. Kiverin, M.A. Liberman. Flame acceleration and DDT of hydrogenoxygen gaseous mixtures in channels with no-slip walls. International Journal of Hydrogen Energy, 36 (13), 7714-7727, 2011.

[188] E. Dziemińska, A.K. Hayashi. Auto-ignition and DDT driven by shock wave-boundary layer interaction in oxyhydrogen mixture. International Journal of Hydrogen Energy, 38 (10), 4185-4193, 2013.

[189] T. Machida, M. Asahara, A.K. Hayashi, N. Tsuboi. Three-dimensional simulation of deflagration-to-detonation transition with a detailed chemical reaction model. Combustion Science and Technology, 186 (10-11), 1758-1773, 2014.

[190] M. Fukuda, E. Dzieminska, A.K. Hayashi, E. Yamada, N. Tsuboi. Effect of wall conditions on DDT in hydrogen-oxygen mixture. Proceedings of the 23rd International Colloquium on the Dynamics of Explosion and Reactive Systems, Paper 207, 2011.

[191] M.F. Ivanov, A.D. Kiverin, I.S. Yakovenko, M.A. Liberman. Hydrogen-oxygen flame acceleration and deflagration-to-detonation transition in three-dimensional rectangular channels with no-slip walls. International Journal of Hydrogen Energy, 38 (36), 1642716440, 2013.

[192] L. Kagan, M. Liberman, G. Sivashinsky. Effect of hydraulic resistance and heat losses on the deflagration-to-detonation transition. In "Pulse Detonation Engine", Moscow, Torus Press Ltd, pp. 51-62, 2006.

[193] V. Gamezo, E. Oran. Flame acceleration in narrow tubes: effect of wall temperature on propulsion characteristics. The $44^{\text {th }}$ AIAA Aerospace Sciences Meeting and Exhibit, Reno, NV, Jan 9-12, 2006. 
[194] J.D. Ott, E.S. Oran, J.D. Anderson Jr. A mechanism for flame acceleration in narrow tubes. AIAA Journal, 41 (7), 1391-1396, 2003.

[195] C.L. Hackert, J.L. Ellzey, O.A. Ezekoye. Effects of thermal boundary conditions on flame shape and quenching in ducts. Combustion and Flame, 112 (1-2), 73-84, 1998.

[196] I. Brailovsky, G. Sivashinsky. Hydraulic resistance as a mechanism for deflagration-todetonation transition. Combustion and Flame, 122 (4), 492-499, 2000.

[197] D. Norton, D. Vlachos. Combustion characteristics and flame stability at the microscale: a CFD study of premixed methane/air mixtures. Chemical Engineering Science, 58 (21), 4871-4882, 2003.

[198] J. Daou, M. Matalon. Influence of conductive heat-losses on the propagation of premixed flames in channels. Combustion and Flame, 128 (4), 321-339, 2002.

[199] G.P. Gauthier, J.M. Bergthorson. Effect of external heat loss on the propagation and quenching of flames in small heat-recirculating tubes. Combustion and Flame, 173, 27-38, 2016.

[200] M. Silvestrini, B. Genova, G. Parisi, F.J. Leon Trujillo. Flame acceleration and DDT run-up distance for smooth and obstacles filled tubes. Journal of Loss Prevention in the Process Industries, 21, 555-562, 2008.

[201] R.W. Houim, E.S. Oran. Structure and flame speed of dilute and dense layered coal-dust explosion. Journal of Loss Prevention in the Process Industries, 36, 214-222, 2015.

[202] K. Seshadri, A.L. Berlad, V. Tangirala. The structure of premixed particle-cloud flames. Combustion and Flame, 89, 333-342, 1992.

[203] Y. Xie, V. Raghavan, A.S. Rangwala. Study of interaction of entrained coal dust particles in lean methane-air premixed flames. Combustion and Flame, 159, 2449-2456, 2012.

[204] W. Zheng, C.R. Kaplan, R.W. Houim, E.S. Oran. Flame acceleration and transition to detonation: effects of a composition gradient in a mixture of methane and air. Proceeding of the Combustion Institute, 37, 3521-3528, 2019.

[205] T. Ogawa, V.N. Gamezo, E.S. Oran. Flame acceleration and transition to detonation in an array of square obstacles. Journal of Loss Prevention in the Process Industries, 26, 355-362, 2013.

[206] V. Bychkov, D. Valiev, L.-E. Eriksson. Physical mechanism of ultrafast flame acceleration. Physical Review Letters, 101, 164501, 2008.

[207] D. Valiev, V. Bychkov, V. Akkerman, C.K. Law, L.-E. Eriksson. Flame acceleration in channels with obstacles in the deflagration-to-detonation transition. Combustion and Flame, 157, 1012-1021, 2010. 
[208] E.S. Oran. Numerical tools for mitigation of methane explosions in coal mines. Grant No. AFC215-20. The Alpha Foundation for the Improvement of Mine Safety and Health, Incorporation, Philadelphia, Pennsylvania, USA, 2017.

[209] Paramagnetic cells technology for our paramagnetic $\mathrm{O}_{2}$ analyzer. (n.d.). Retrieved from https://www.systechillinois.com/en/support/technologies/paramagnetic-cells.

[210] ImageJ. Retrieved from https://imagej.nih.gov/ij/.

[211] Keisan Online Calculator. From https://keisan.casio.com/exec/system/1223372110.

[212] V. Akkerman, C.K. Law, V. Bychkov. Self-similar accelerative propagation of expanding wrinkled flames and explosion triggering. Physical Review E, 83, 026305, 2011.

[213] V. Akkerman, V. Bychkov. Velocity of weakly turbulent flames of finite thickness. Combustion Theory and Modelling, 9, 323-351, 2005.

[214] P. Pelce, P. Clavin. Influence of hydrodynamics and diffusion upon the stability limit of laminar premixed flames. Journal of Fluid Mechanics, 124, 219-237, 1982.

[215] Y. Gostintsev, A. Istratov, Y. Shulenin. Self-similar propagation of a free turbulent flame in mixed gas mixtures. Combustion, Explosion and Shock Waves, 24, 563-569, 1988.

[216] D. Bradley, T. Cresswell, J.S. Puttock. Flame acceleration due to flame-induced instabilities in large-scale explosions. Combustion and Flame, 124, 551-559, 2001.

[217] V.V. Molkov, D.V. Makarov, H. Schneider. Hydrogen-air deflagrations in open atmosphere: large eddy simulation analysis of experimental data. International Journal of Hydrogen Energy, 32, 2198-2205, 2007.

[218] W.K. Kim, T. Mogi, K. Kuwana, R. Dobashi. Self-similar propagation of expanding spherical flames in large-scale gas explosions. Proceedings of the Combustion Institute, 35, 2051-2058, 2015.

[219] G. Jomaas, C.K. Law, J. Bechtold, On transition to cellularity in expanding spherical flames. Journal of Fluid Mechanics, 583, 1-26, 2007.

[220] S.G. Davis, J. Quinard, G. Searby. Markstein numbers in counterflow, methane- and propane- air flames: a computational study. Combustion and Flame, 130, 123-136, 2002.

[221] A. Adebiyi, R. Alkandari, D. Valiev, V. Akkerman. Effect of surface friction on ultrafast flame acceleration in obstructed cylindrical pipes. AIP Advances, 9, 035249, 2019.

[222] P. Taylor, S.J. Bimson. Flame propagation along a vented duct containing grids. Proceedings of the Combustion Institute, 22, 1355-1362, 1988.

[223] C. Morley, GASEQ - A Chemical Equilibrium Program, 2005. http://www.gaseq.co.uk/ 
[224] S. Demir, H. Sezer, T. Bush, V. Akkerman. Promotion and mitigation of premixed flame propagation in a gaseous-dusty environment with various dust distributions. Fire Safety Journal, 105, 270-276, 2019.

[225] V. Akkerman, D. Valiev. Moderation of flame acceleration in obstructed cylindrical pipes due to Gas Compression. Physics of Fluids, 30, 106101, 2018.

[226] F. Kodakoglu, V. Akkerman. Analytical study of an effect of gas compressibility on a burning accident in an obstructed passage. Physics of Fluids, 32, 074602, 2020.

[227] V. Akkerman, C.K. Law. Effect of acoustic coupling on power-law flame acceleration in spherical confinement. Physics of Fluids, 25, 013602, 2013.

[228] D. C. Haworth. Progress in probability density function methods for turbulent reacting flows. Progress in Energy and Combustion Science, 36 (2), 168-259, 2010.

[229] H. Wei, X. Zhang, H. Zeng, R. Deiterding, J. Pan, and L. Zhou. Mechanism of end-gas autoignition induced by flame-pressure interactions in confined space. Physics of Fluids, 31 (7), 076106, 2019.

[230] V. Bychkov, V. Akkerman, D. Valiev, C.K. Law. Role of compressibility in moderating flame acceleration in tubes. Physical Review E, 81, 026309-1-9, 2010.

[231] V. Bychkov, V. Akkerman, D. Valiev, C.K. Law. Influence of gas compression on flame acceleration in channels with obstacles. Combustion and Flame, 157, 2008-2011, 2010.

[232] M. Kuznetsov, G. Ciccarelli, S. Dorofeev, V. Alekseev, Yu. Yankin, T.H. Kim. DDT in methane-air mixtures. Shock Waves, 12, 215-220, 2002. 